\title{
The emergent realities of project praxis in socially complex project environments
}

A thesis submitted in partial fulfilment of the requirements for the degree of Doctorate of Project Management

Jocelyn M. Small

Dip.App.Sc (Nsg), Grad.Cert.Midwifery, Dip.Hom., BN(Honors), Grad.Dip.Ed., M.NS., M.Ed.

School of Property, Construction and Project Management

RMIT University

March 2009 
Declaration

I certify that except where due acknowledgement has been made, the work is that of the author alone; the work has not been submitted previously, in whole or in part, to qualify for any other academic award; the content of the thesis is the result of work which has been carried out since the official commencement date of the approved research program; any editorial work, paid or unpaid, carried out by a third party is acknowledged; and, ethics procedures and guidelines have been followed.

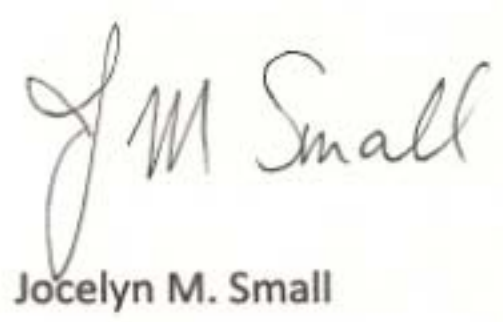

ii 


\section{Acknowledgements}

There are a number of people that need to be acknowledged for their support and guidance freely given to me throughout my Doctoral journey over the past four years.

First and foremost, Professor Derek Walker whose passion for project management in all its guises has been, and will always be, inspirational and motivational. Derek's skills in facilitating the human interconnections necessary for a global community of project management doctoral students, researchers and practitioners, have been instrumental in broadening the theoretical and practical understandings of project actuality. Derek's guidance and gifted technique of giving me 'just the right amount of rope' have been pivotal in my conceptualising and finalising this dissertation.

I would also like to thank Adjunct Professor Kersti Nogeste, who over the final 12 - 18 months in particular, has provided the project management expertise necessary to ensure I have kept on track. Kersti's attention to detail and logical approach to writing have been invaluable in ensuring that my concepts are clearly presented and articulated.

The other most significant person I need to acknowledge is Jim, my husband and partner on this journey. His immense understanding and patience whilst I worked through different stages of the doctoral program, accompanying me to far-flung countries so I could present my latest thoughts, and keeping us afloat financially as I took some time out from the paid workforce to finalise writing, are all elements integral to this dissertation having finally being completed. In addition, I need to acknowledge my children for their contributions: my daughter Melanie for using her computer graphics skills to help me convert my pencil sketches to an electronic format; my son Joel for his genuine interest and discussions about the conceptual underpinnings of my research; and my other daughter Jodi, who kept asking when was I going to be finished!

Finally, I would like to dedicate this dissertation in memory of my father who passed away before I could show him the finished research product. He read with interest my initial course work and conference papers, making valuable comment and suggestions over the first couple of years of the program. My determination to finish what I had started a few years ago is credit to his lifetime of intelligent guidance and the hours spent working through schoolwork with me when I was younger. Dad never gave me answers freely, rather he encouraged me to critically think and question to find a solution. Life skills that I hope I have passed on to my children. 


\section{Publications and conference presentations during Doctorate of Project Management program}

Small, J. (2006). Leaders, Learners and Laggards: Capacity planning for organisational effectiveness. Quality Assurance in Higher Education: Mission (Im)possible?, Pretoria, South Africa http://www.fotim.ac.za/fotim/fotim conferences/qaconference2006/papers/paper small.pdf

Small, J. (2006). The Leadership Conundrum: When power and politics interfere in the management of social capital. The 6th International Conference on Knowledge, Culture \& Change in Organisations, Prato, Italy http://ijm.cgpublisher.com/product/pub.28/prod.495 Common Ground publishing. pp 141-148

Small, J. (2007). Reconciling an organisation's learning capabiity with knowledge strategy formation in a project/program management setting: A model to bridge the normative and descriptive divide in capacity planning for sustainable advantage. ESC Lille/UTS Doctoral seminar on research in strategy, project and programme management. Lille, France. 


\section{Table of Contents}

Table of Contents

$\boldsymbol{v}$

Index of Figures ___ ix

Index of Tables __ $x$

Summary of research __ 1

Chapter 1 Introduction __ 4

1.1 Introduction overview __ 4

1.2 Doctorate of Project Management

1.3 The Research Context __ 8

1.4 The problematical situation 10

1.5 Research aims _ 11

1.6 Conceptual and philosophical framework _ 12

1.7 Methodology _ 13

1.8 Scope of the research study

1.9 Structure of dissertation

1.10 Chapter summary __ 16

Chapter 2 Literature Review__ 18

2.1 Introduction _ 18

2.2 Research philosophical \& conceptual framework _ 22

2.3 Knowledge \& learning for change___ 27

2.3.1 Consciousness and cognition for sensemaking __ 27

2.3.2 The pragmatism of knowledge___ 34

2.4 The changing nature of organizations 36

2.4.1 The cognitive nature of the knowledge based organization __ 36

2.4.2 The project organisation __ 43

2.4.3 Organizational capacity to learn

2.4.4 Quality, effectiveness and the resilient organization___ 56

2.5 The complexity of context___ 63

2.5.1 The biological nature of social complexity __ 63

2.5.2 Human embeddedness and the influence of language ___ 67

2.5.3 The realities of culture and religion __ 70

2.5.4 The interconnect of knowledge with politics and power 79

2.5.5 Projects as complex human activity systems ___ 87

2.6 Chapter Summary ___ 94 
3.1 Introduction _ 98

3.2 The Problematical Situation __ 101

3.3 The choice of paradigm _ 102

3.3.1 Hard Systems or Soft Systems? 103

3.3.2 Positivist, Interpretivist or Critical Thinking? __ 109

3.4 The research conceptual framework

3.5 The choice of research approach _ 117

3.5.1 Evaluation, Action or Impact assessment?___ 117

3.5.2 Qualitative or quantitative? __ 119

3.5.3 The purpose - exploratory, descriptive or explanatory? __ 122

3.6 The research methodology 126

3.6.1 Section introduction__ 126

3.6.2 Soft Systems Methodology (SSM) - The methodology and rationale __ 126

3.6.3 SSM and project practice ___ 128

3.6.4 Soft Systems Methodology - methodology or method? ___ 129

3.6.4.1 Cycle One and Two (SSMP) - The beginnings of the inquiry process ___ 132

3.6.4.2 Cycle One and Two - The Finding Out through making 'Rich Pictures' ___ 133

3.6.4.3 Cycle Three (SSMC) _ 134

3.6.4.3.1 The finding out through rich pictures ___ 134

3.6.4.3.2 The Finding Out - focussing on the intervention itself (Analysis One) ___ 135

3.6.4.3.3 The Finding Out - through Cultural Analysis (Analysis Two)__ 136

3.6.4.3.4 The Finding Out - through Political Analysis (Analysis Three)___ 137

3.6.4.3.5 Making purposeful activity models _ 137

3.6.4.3.6 Using the models for questioning and discussions __ 139

3.6.4.3.7 Defining - as opposed to taking - action__ 139

3.6.4.3.8 Reflecting upon the situation - rather than action (SSMc - SSMP) __ 140

3.7 The issues of rigour 140

$3.8 \quad$ Ethical considerations __ 144

3.9 Chapter Summary __ 149

Chapter $4 \quad$ The SSM Learning Cycle in Action ___ 151

4.1 The Situation _ 151

4.1.1 Introduction _ 151

4.1.2 The Context _ 152

4.1.3 The Problem _ 157

4.2 The Learning Cycles in Action __ 161

4.2.1 Introduction _ 161

4.2.2 Cycle $1 \ldots 161$

4.2.3 Cycle $2 \longrightarrow 167$

4.2.4 Cycle $3 \longrightarrow 175$

4.2.4.1 The Finding Out 175

4.2.4.1.1 Rich Picture 175

4.2.4.1.2 Analysis $1 \ldots 177$ 
4.2.4.1.3 Analysis 2 178

4.2.4.1.4 Analysis $3 \ldots 181$

4.2.4.2 Building Purposeful Activity Models _ 184

4.2.4.2.1 Expressing the problematic situation - The Generic Model __ 184

4.2.4.2.2 Purposeful Activity Model One___ 187

4.2.4.2.3 Purposeful Activity Model Two___ 189

4.2.4.2.4 Purposeful Activity Model Three __ 191

4.2.4.2.5 Purposeful Activity Model Four __ 193

4.2.4.2.6 Purposeful Activity Model Five___ 195

4.2.4.3 Discussing the situation to generate ideas _ 197

4.2.4.4 Defining desirable and feasible change ___ 197

4.2.5 Taking Action _ 199

4.2.6 Cycle $4 \ldots 202$

4.3 Summary and conclusion __ 204

Chapter 5 Discussion and conclusions __ 206

5.1 The significance of the project context___ 206

5.1.1 Introduction __ 206

5.1.2 The influence of the socio-cultural environment___ 208

5.1.3 Language, communication and cognition __ 222

5.1.4 Enacting cognition: Barriers, constraints and leadership___ 226

5.1.4.1 Knowledge as enacted cognition __ 227

5.1.4.2 Contextual constraints and barriers__ 228

5.1.4.3 Complex power games___ 232

5.1.4.4 The politics of tribalism _ 233

5.1.4.5 Leadership as the way forward _ 237

5.2 Projects as social process __ 240

5.2.1 The complexity of difference ___ 241

5.2.2 Project management strategy ___ 244

5.3 Limitations ___ 252

5.4 Recommendations___ 253

5.5 Conclusion ___ 254

Glossary of Terms___ 261

References __ 266

Appendix 1 _ 293

College PQA Recommendations - Summary___ 293

Appendix 2

College QA Planning Group Feedback (26/10/05) ___ 295

Appendix $3 \ldots 297$

College QA 2005-2006 timeline___ 297

Appendix $4 \ldots 298$

College QA Data Checklist ‘05-`06 Academic Chairs __ 298 
Appendix 5 302

College QA Data Responsibilities '05-'06 Chairs \& Program Staff 302

Appendix 6 304

College Data Management Responsibilities 2005-2006 304

Appendix 7 306

The problem situation themes 306

Appendix 8 314

Cycle 4 data from informal meetings and email responses 314 


\section{Index of Figures}

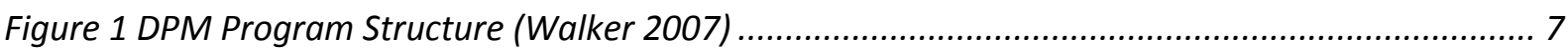

Figure 2 DPM Contribution to PM Practice of Theory (Walker 2007) ............................................... 8

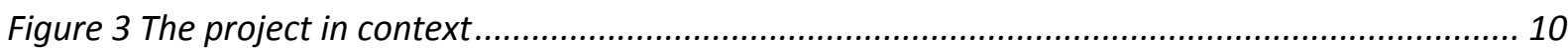

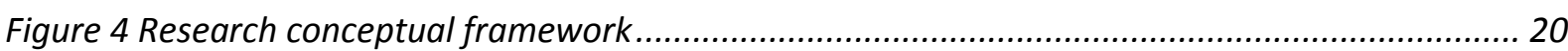

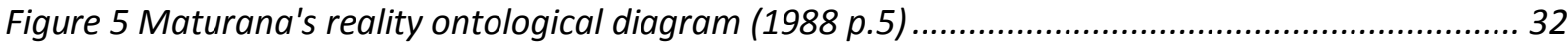

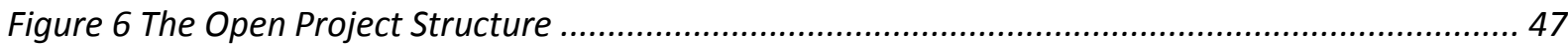

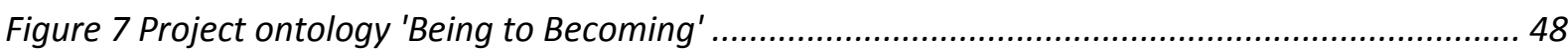

Figure 8 Autopoietic organisational learning model (sourced Parboteeah \& Jackson, 2007 p257) ..... 51

Figure 9 The core process of becoming aware (sourced Scharmer \& Varela 2000 p.4)...................... 53

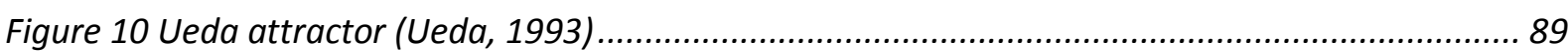

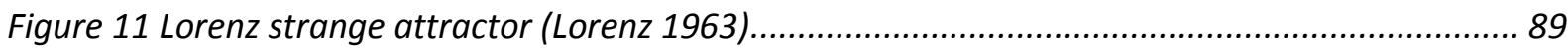

Figure 12 The research activity as a knowledge creation process (adapted from Cicmil, 2006 p.29) 103

Figure 13 Viewing the world (adapted Checkland and Scholes, 1990 p.A11) ................................. 108

Figure 14 Project Management and paradigms (sourced Pollack, 2007 p.267) ............................... 110

Figure 15 SSM - the methodological process. Adapted from Checkland and Scholes (1990) p.29 ..... 131

Figure 16 Incorporating current problem situation into SSM's basic process ................................... 132

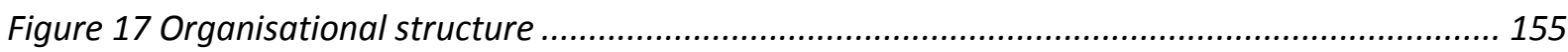

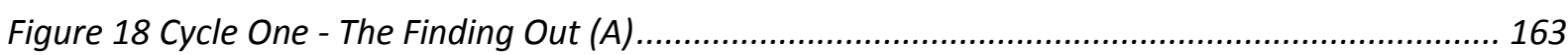

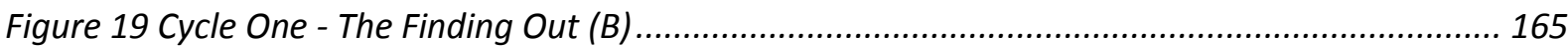

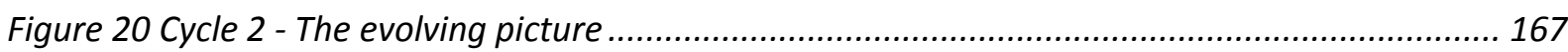

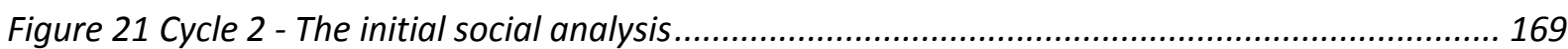

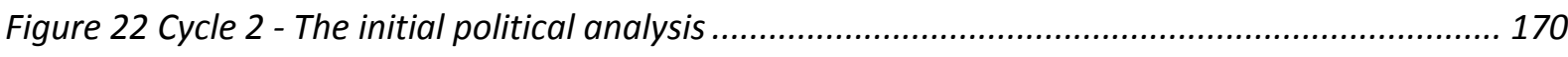

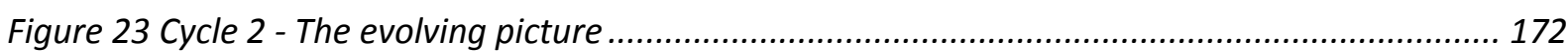

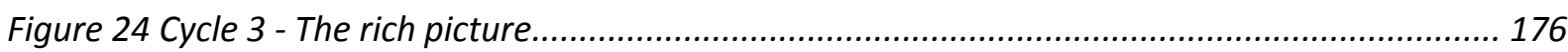

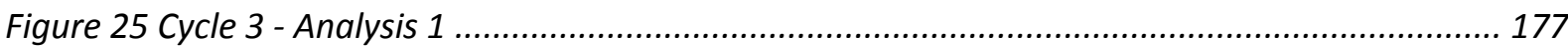

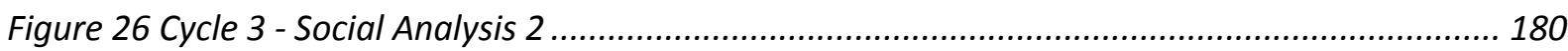

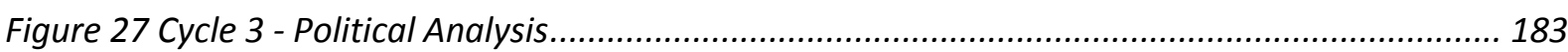

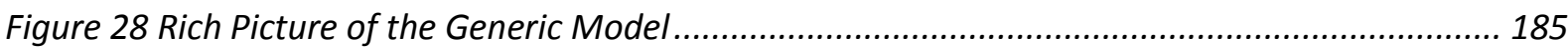

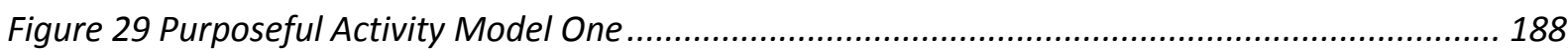

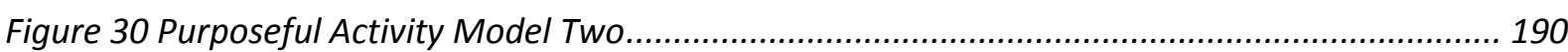

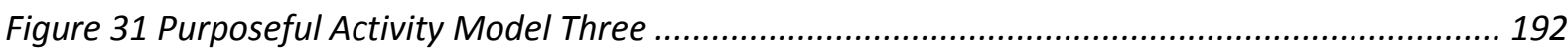

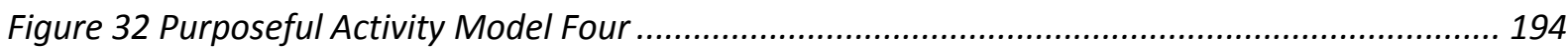

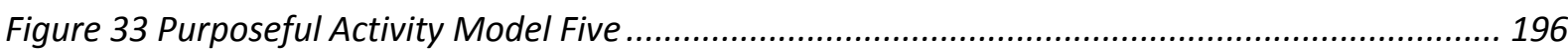

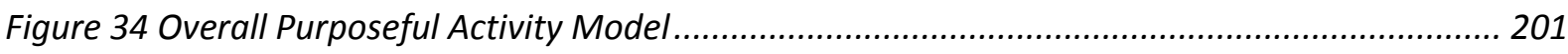

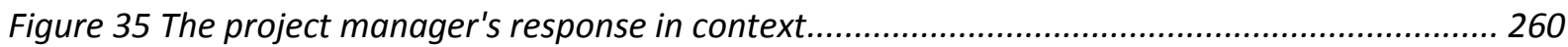




\section{Index of Tables}

Table 1 Comparing complex theoretical assumptions

Table 2 Directions for Future Research (sourced Winter, Smith et al 2006, p.642)

Table 3 Comparing Enactivism with other dominant approaches to social research (adapted Neuman, 2006 p.105)

Table 4 Purpose and Methods of Research (adapted Neuman 2006 p34 \& Richey \& Klein 2007 p44)

Table 5 The Learning Cycles in Action ......

Table 6 Cycle One Action Matrix (structure modelled on Checkland \& Scholes 1990). 160

Table 7 Cycle Two - Action Matrix 166

Table 8 Matrix comparing activity models...... 174

Table 9 Linking desirability and feasibility with action 198

Table 10 Factors affecting organisational effectiveness 199

Table 11 Comparing Problem Owner leadership preferences with project GLOBE results 203 239 


\section{Summary of research}

The research as described in this dissertation has aimed to explore the significance of the sociocultural context, socio-technical infrastructure and human capital elements relevant to managing projects for effective organizational change. Complexity in project practice and theory has traditionally focussed on the technical elements of project practice. The following research has aimed to broaden this view by moving beyond instrumentality through conceptualising the social nature of systems where the ecology of the project entity entails a complexity which is socially derived. Such thinking supports associating organisational resilience with the capacity of a project organisation to mutually adapt to, and create the environmental context in which project practice is embedded. Given these realities, the second aim in this research has been to explore the feasibility of developing project management strategy that can accommodate for diversity and difference to facilitate the cognitive emergence that underpins learning and change.

The research has been implemented in a higher education institute in the Middle East and eventuated subsequent to encountering a problematic situation in project practice. Lack of progress in implementing a Quality Assurance system within the organisation initiated an exploration into what was perceived as an ill-defined and messy problem. Soft Systems Methodology (SSM) was therefore selected as the process of enquiry due to its flexible methodology providing guiding principles that can potentially bring about improvement in a situation. The research process has involved exploring the different worldviews of stakeholders to ascertain their perceptions of the problematical situation and given the socio-cultural mix of expatriate stakeholders in the project organisation appreciation of disparities in understanding emerged as significant.

The project organisation was bureaucratically-structured, with an Arabic system of governance and accountability influencing operational ways of doing business. The overlay of an externally driven system of authority was seen to perpetuate a number of tensions that were identified as originating from the social inequalities and power imbalances existing. Issues of autonomy in project practice contributed to the emerging awareness of the social nature of project complexity. The mix of expatriate staff working within the organisation, the discursive nature of communication and the political nature of the environmental context interconnected as factors needing to be appreciated.

A research and philosophical conceptual framework has been defined to guide the enquiry, with enactivism and postmodern thinking being incorporated into the theoretical underpinnings of the research. An assumed pragmatic epistemology and becoming ontology support the notion of project 
praxis being messy, non-linear and dynamic, to better reflect a project actuality that involves social processes that entail a constant flux of events.

Power to effect relationships that foster a mutuality and reciprocity in project practice has been identified in this research as being critical to project outcomes. The nature of power games perceived as existing and sustaining Arabic systems of control within the project organisation revealed the need for different project management responses to balance the situational dynamics given the sociopolitical contextual factors. Although the second aim in this research was to develop a project management technique to accommodate for difference given the social complexities existing in the project environment, what subsequently emerged throughout the enquiry process was the greater desirability in defining a project manager's approach or response to circumnavigate the competing pressures with and within the project context. Attempting to define a prescriptive project management model or tool to deal with social complexity emerged as not only being undesirable given the adaptive nature in the actuality of projects, but was considered as perpetuating the more rational and positivist approaches to project management.

The research in this dissertation aimed to address some of the limitations identified in the project management literature, through defining project practice within an ecological framework to better highlight the social process nature of project practice. This research has suggested that the project entity provides the structural openness for organisations to sense and contribute to environmental change to move beyond self-regulatory adaptive behaviour, to a state of constantly evolving resilience. Structurally coupled relationships of all elements as integral to project practice provide the interconnections within a context to facilitate the cognition that emerges from complexes of multiple realities.

The complex nature of project practice has therefore been identified as involving adaptive responses to accommodate for ambiguities and conflicting understandings resulting from changing realities being encountered in today's world of business by projects. The findings in this research have concluded that integral to the role of an effective project manager, is the presence of a profound ability to shape the project context from within. Facilitating the interconnections of human multiple realities despite differences in language and understandings necessitates the application of adaptive systems of governance to enable the enactment of cognition to bring forth new worlds of culturally patterned existence and meaning. Difference and diversity can be a strength in project practice, but balancing the contextual dynamics for some semblance of consensus in action, will be in part dependent upon acknowledging the inherent social complexities as the project actuality and in part having the PM skills to be flexible and adaptive in response. 
"Good theories are flexible. Those which have a rigid form and which can not change that form without colfapsing realfy have too fittle vitafity... G. Good theories can respond to alf ofjections. Specious arguments fiave no effect on them, and they also triumph over alf serious ofjections. Flowever, in triumphing they may be transformed"

$\left(\mathscr{P}_{\text {oincaré 1900 }}\right)$ 


\section{Chapter 1 Introduction}

\subsection{Introduction overview}

Project management as a social practice and way of doing business, is being evidenced globally as a critical factor in organisational innovation and change (Cicmil and Hodgson 2006). The emergent nature of organizations reflects the actuality of social transformation in an era of increasing complexity, with project practice demonstrating the potential to facilitate organisational learning for adaptive resilience. The research described in this dissertation was initiated subsequent to challenges encountered when implementing a quality assurance (QA) system in an educational institute in the Middle East. Difficulties faced were perceived as a problematical situation warranting exploration in order to unravel some of the inherent ambiguities and messiness.

The research dissertation is in part fulfilment of a RMIT Doctorate of Project Management (DPM) (1.2). After grappling with a number of issues related to the QA project in the workplace, I found that the DPM program enabled a more structured approach to exploration through providing the opportunity to consider a number of possible factors contributing to the contextual project issues.

The research project context (1.3) was at a single College campus of a higher educational institute comprising 11 other campuses throughout the United Arab Emirates (UAE). The organisation was Arabic governed and staffed primarily by a diverse expatriate population comprising faculty and staff sourced globally for three-year contracts. My primary role in the project organization was Chair of an academic department, but throughout a large proportion of the DPM research project, I also held the position of College QA co-ordinator. Details of the project organisation are elaborated upon in sections 3.2 and 4.1.2, but in summary, it was an organisation established about 18 years prior, to specifically provide higher education to Emirati national students by equipping them with the 'ability, skills and potential to make the fullest possible contribution to the development of the community'. In the period of my employ, the Institute commenced a process of seeking external international organisational accreditation, and integral to this application was the establishment of College based Program Quality Assurance (PQA) initiatives.

Quality improvement programs have been aligned with organisational change and effectiveness for transformation (Bass and Avolio 1994), but this assumption is based on an understanding that the cyclic nature of quality systems will involve interconnected evaluative processes to underpin strategic change. Implementation of the QA system in the research project organisation was a new initiative that was seemingly being imposed upon, rather than dovetailed into, existing program processes. This gave the impression of a QA system running parallel to teaching and learning 
delivery, rather than being interconnected as would be necessary for effective evaluation. The problematical situation (1.4) thus emerged as the quality assurance project progressed. Reflective exploration ensued with two key factors seemingly arising as critical elements in the management of projects in culturally ambiguous and socially complex working environments. As researcher and 'problem solver', my perception of the real-world situation seemed to indicate the inherent human element as critical to project outcomes. This factor when embedded within a workplace comprising a social diversity and pluralism, from my perspective, called for recognition and strategic management to facilitate the necessary emergent cognition for organisational change. However to accurately investigate the problem, the 'real-world' constructs of other stakeholders needed to be explored to gain a richer picture of the problematical situation and to ascertain the desirability and feasibility of change.

Discontinuous and fragmented project practice does not support desired change, and quality improvement programs disconnected from the realities of organisational functioning highlight the importance of bridging the divide between organisational realities and future transformation (Engeström, Kerosuo et al. 2007b). Exploration into the actuality of the situation appeared to have the dual action of sifting through some of the situational uncertainties to bring about improvement, together with potentially contributing to broadening the theoretical base of project management. Organizational theory and project management practice have traditionally concentrated on rational and structural aspects of functionality for improved project outcomes (Söderlund 2004a; Atkinson, Crawford et al. 2006; van Donk and Molloy 2008). However, such frameworks inadequately facilitate stakeholder 'sense-making' within a project environment, failing to address many of the conflicting issues involving culture and power relations in the creation and assimilation of knowledge. In turn, the perpetuation of reifying 'knowledge' as a commodity that can be 'transferred', 'codified' and 'controlled', supports a structural approach to cognitive awareness which is at odds with the realities of emergent and embodied cognition in context for organisational resilience (Riegler 2002; Folke 2006; Proulx 2008).

The research in this dissertation therefore aims to explore socio-cultural factors perceived by stakeholders as relevant to the problematical situation, to facilitate a shift in thinking from projects as instrumental processes towards viewing projects as social process. The research aims to contribute to the project management theory for improved project outcomes noting complexity arising from socially disparate work environments (1.5). The 'finding out' about the situation included both formal and informal data collection and involved note taking from thoughts and reflections on conversations with faculty and management, identification of relevant items from business discussed in meetings, and unstructured interviews with fifteen members of the College's management team. Rich pictures, as integral to Soft Systems Methodology (SSM) (1.7) evolved 
based on my perceptions, the real-world constructs of other stakeholders, together with comparison and reflection on the overall situation.

As described in section 1.6, a philosophical and conceptual framework has been defined to embrace thinking underpinning the exploratory research in this dissertation. Projects are viewed as social process involving context dependent intersubjective relationships and multiple realities (Pettigrew 1987; Green 2006; Winter, Smith et al. 2006), as human activity systems embedded in context as autopoietic, self-organising, complex living systems (Checkland and Scholes 1990; Engeström 2001), and as being messy, in a state of flux and non-linear (Winter and Checkland 2003). Such thinking is supported by a 'becoming' ontology (Chia 1995; Lineham and Kavanagh 2006) and a pragmatic epistemology (Peirce 1868; Dewey 1929; James 1975; Calori 2002), with an enactivist philosophical framework being drawn upon to critically appraise the socially complex nature of project practice.

SSM (1.7) has been selected as the approach to guide the social enquiry to bridge the normative and descriptive research divides which tend to exist in traditional methods of social research (Robinson 2001). SSM also serves to accommodate for the assumed embedded, interconnected, non-static nature of humans as complex inquiring beings to facilitate an active approach for emergent cognition, to bring about effective change and organisational transformation.

The scope of the study (1.8) involves a shift in philosophical thought from more positivist structural views of project praxis, to viewing the complex social nature of projects as the real actuality. Projects considered as emergent, non-linear and contextually dependent will not only challenge the traditional more static portrayal of project practice, but will facilitate a re-think on management competencies and strategies required for success in today's project environment (Cicmil, Williams et al. 2006). Projects in a social context comprising individuals of disparate cultural and religious backgrounds create a complexity of ambiguities and paradoxes. As has been noted by Winter, Smith et al (2006), “...the current conceptual base of project management continues to attract criticism for its lack of relevance to practice ..." (p. 638). Thus, there is an imperative to embrace different approaches to project management research so that the human element and the role actors play in constructing and relating within specific contexts can be configured.

\subsection{Doctorate of Project Management}

The following Research Dissertation is submitted as the final component of the Royal Melbourne Institute of Technology (RMIT) University Doctorate of Project Management (DPM), details of which can be found at URL:

http://dhtw.tce.rmit.edu.au/pmgt/\#LINK7 
The DPM is a three to four year program that combines approximately one third of online course work with two-thirds research. Units of study include Project Management (PM) Knowledge Management, PM Leadership, PM Ethics and Procurement and PM Practice. These units in turn have an associated Reflective Learning course which encourage further exploration of the literature to develop the basis of a research project framework (Figure 1)

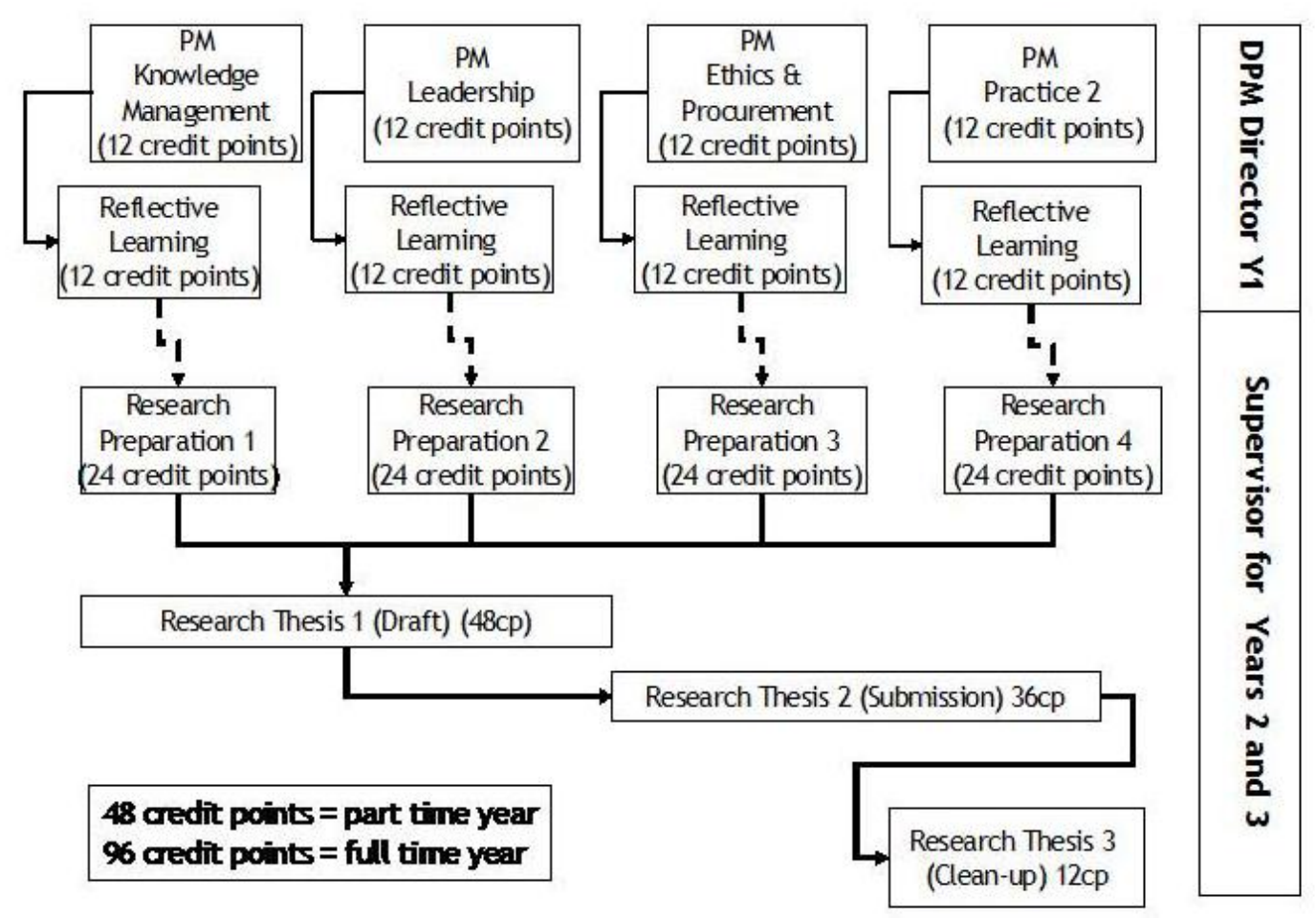

Figure 1 DPM Program Structure (Walker 2007)

The research papers I completed in the DPM courses and reflective learning units explored the role and relevant styles of leadership in PM, knowledge management and transfer systems, barriers to organisational effectiveness, specific workplace project case study scenarios (including the quality improvement project detailed in this dissertation) and the effects of complex socio-cultural work environments on organisational learning and effectiveness. The literature research and reflective papers thus underpinned and contributed to the dissertation development following the Project Management DPM Practice to Theory framework presented in Figure 2. 


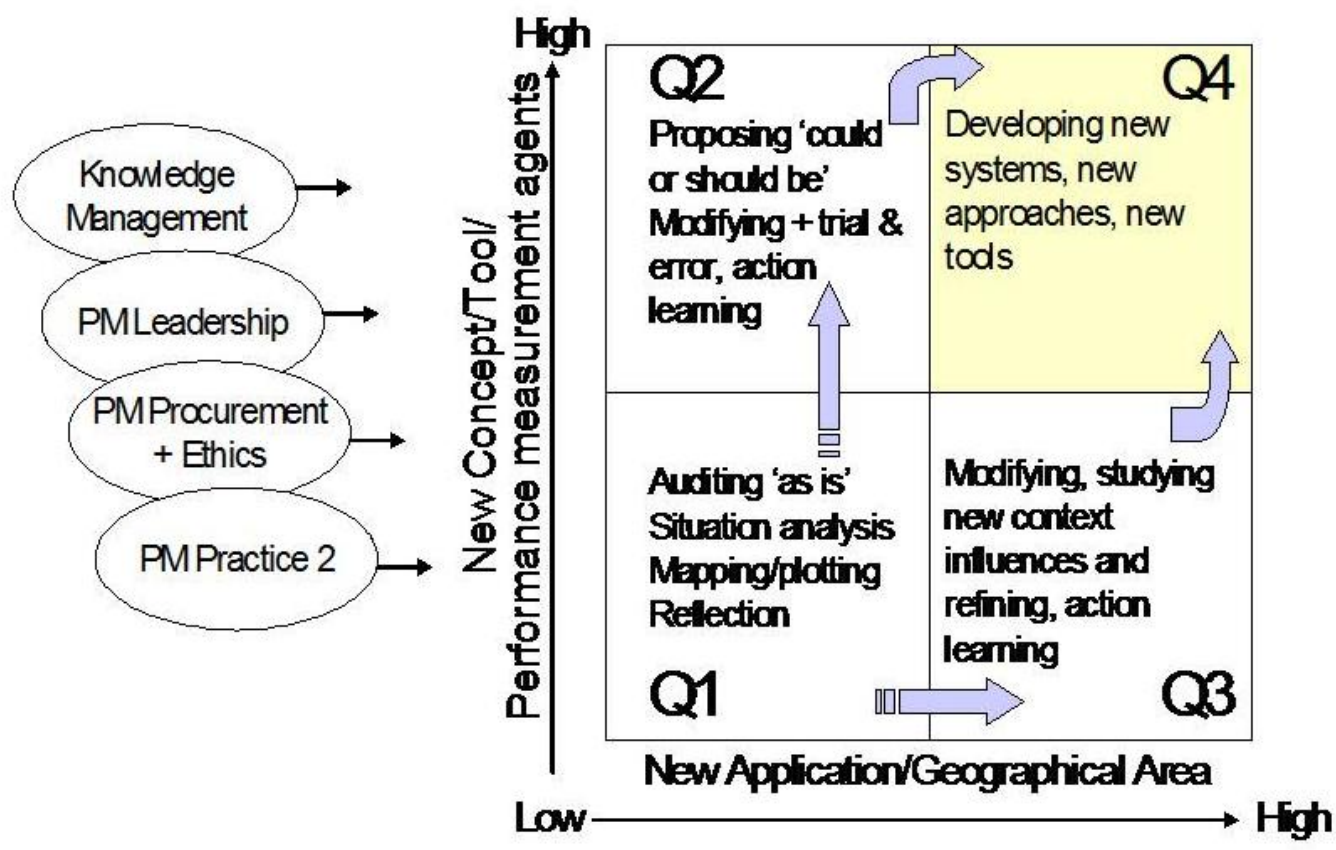

Figure 2 DPM Contribution to PM Practice of Theory (Walker 2007)

Although the implementation of a quality improvement system in an educational institution in the Middle East was the initiating factor in the project study, the problematical situation and subsequent investigations were found to be relevant to a much wider selection of social contexts. Project organisations that particularly rely on transient human capital that is of a culturally diverse nature are faced with a social complexity that necessitates strategic management for effective outcomes. In reference to the above Figure 2 detailing DPM contribution to PM Practice, it is considered that the following dissertation focuses on the $3^{\text {rd }}$ quadrant through studying a new context and influences in project management through action learning, with the work moving toward developing new approaches and systems for PM ( $4^{\text {th }}$ quadrant) through broadening the conceptual base of PM theory.

\subsection{The Research Context}

As noted in the Introduction (1.1), the research context, both country and organisation, are presented in further detail in sections 3.2 and 4.1.2. However, for introductory purposes, the geographical placement of the research context is in the UAE on the Arabian peninsula in the middle east, and more specifically, the research was implemented in a College of higher education within one of the seven UAE emirates. The College was one of 12 of an institute system providing education 
for national Emirati males and females, with the College involved having approximately 2,500 female students enrolled.

The College was staffed mainly by expatriates sourced globally from a variety of countries, with management and faculty predominantly originating from the western countries of UK, USA, Australia, Canada and South Africa and lesser numbers from the Gulf and other Arabic countries. The organisation was Emirati owned and governed and established about 18 years prior to this research project. The language of tuition and business was English with operations functioning within the Islamic legislative framework of the country's national Shari'a law.

Social and contextual complexities existed primarily due to the disparate nature of the organisation's workforce, with ambiguities in understanding and ways of doing being directly attributed to cultural assumptions underpinning communicative practices. Despite the universality of the English language being used, the heterogeneous nature of the workforce was culturally determined, and social groupings were multilayered with religion, country of origin and native language being examples of factors which provided a 'glue' of association. Project teams within the organisation were thus socially discursive with interwoven relationships based on political, personal, position and length of stay with the organisation and country.

An initial pictorial representation of complexity existing within the research project context as perceived by the author of this dissertation is presented in Figure 3 below. However, as the research described in this dissertation progressed, a number of rich pictures were developed from reflections on own perceptions and stakeholder real-world constructs, and through comparing individual models of activity with actual and desirable system activities. The generic situational rich picture evolved and may be seen as Figure 28 in 4.2.4.2.1 and a final diagram, Figure 35, incorporating all identified elements may be found in the conclusion. 


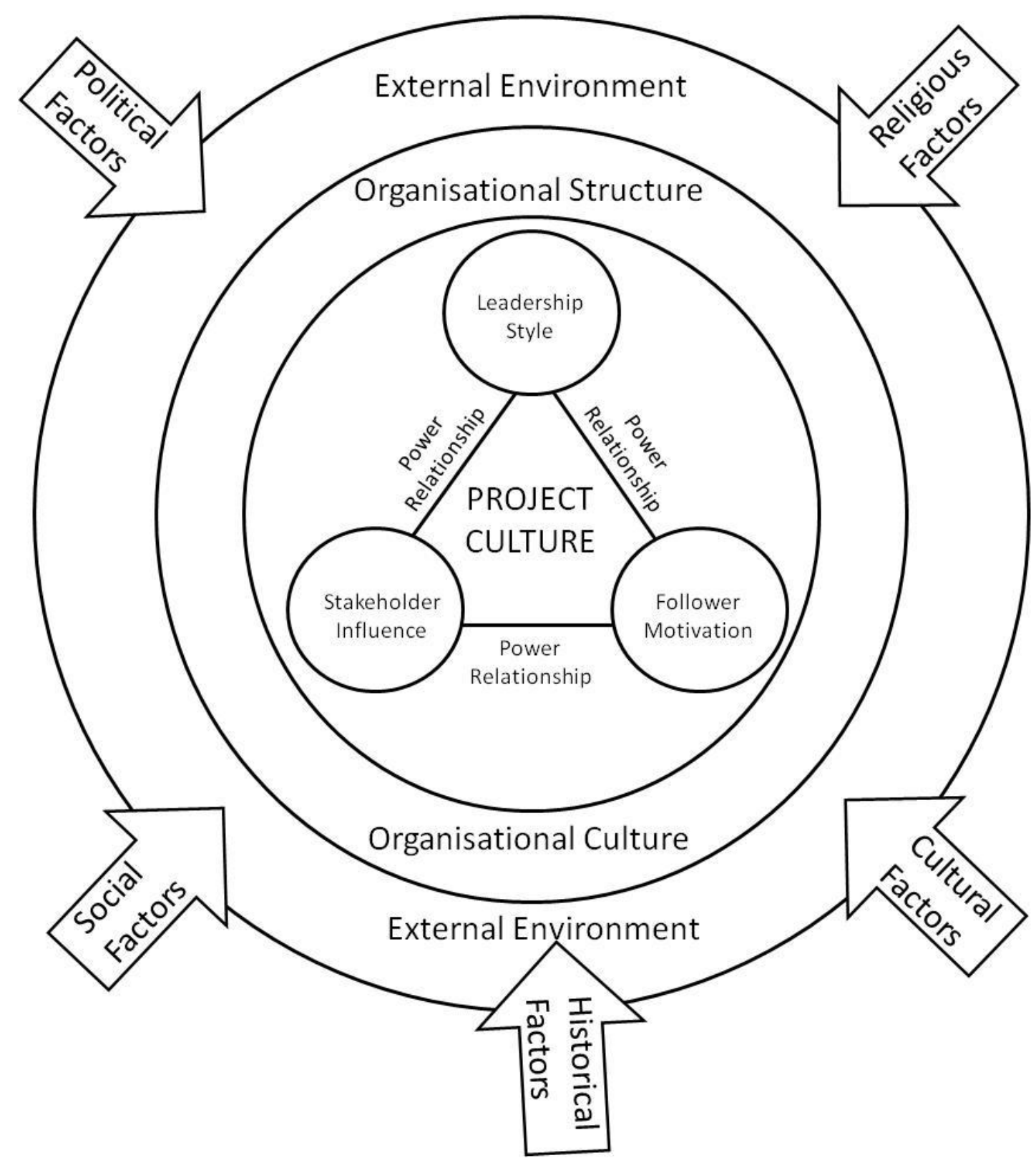

Figure 3 The project in context

\subsection{The problematical situation}

The situation underpinning the research in this dissertation, as described in more detail in sections 3.2 and 4.1.3 arose from ongoing difficulties encountered when managing a new project initiative to implement a Quality Assurance (QA) system in a higher education institute in the UAE. Increasing market competition and the pressure to demonstrate improved performance and community accountability were two of the reasons behind the Institute commencing the process to gain external international accreditation. A centralised Institutional Effectiveness Directorate was established to progress this aim, with the mission being 'dedicated to providing quality instructional, student and 
evaluation services, as well as academic resource and technical support to all campuses'. Implementation of College based QA as part of the accreditation application became the remit of individual colleges within the larger Institutional system, and it is at this level that the project was managed and the research was implemented.

The perceived problematical situation was primarily a lack of project progress, with evidence of competing stakeholder agendas, ambiguities and uncertainties in understanding and a degree of inertia to new systems approaches.

A number of 'how to' questions emerged through a process of reflection on encounters with other stakeholders and discussions as relevant to the QA project. These were:

How to:

i. Implement a new Quality Assurance system when the imperative is externally driven?

ii. Communicate to a population with divergent language patterns about the relationship between quality assurance and organizational effectiveness?

iii. Define and articulate QA processes logically in the absence of any established cyclic improvement pattern?

iv. Accommodate and work with diversity, where ways of knowing and being exist in a culturally complex organization?

v. Work with and manage the tensions that exist due to these predominantly culturally derived differences?

vi. Implement and manage a QA project in a context where planning tends to be short-term focused and expected outcomes are considered changeable, moveable and at times outside human control?

\subsection{Research aims}

These questions in 1.4 (i-vi) then evolved to focus on possible factors that may influence organizational change and how quality improvement programs may interconnect. As this organisation relied primarily upon an expatriate work force, a major challenge was in the creation and strategic management of emergent knowledge for organizational effectiveness due to the transient nature of the human capital. The distributed nature of knowledge which was apparent in the disparate cultural mix of employees, raised issues in relation to how learning could be facilitated given the embodied nature of cognition embedded in context. 
The initial aims of the research project were therefore to:

i. Contribute to organizational learning theory acknowledging cognition being central to change

ii. Determine factors necessary to strategically manage knowledge in culturally complex working environments

iii. Use the implementation of a quality improvement system as the project study and align with organizational effectiveness

iv. Explore the impact/influence of Arabic ways of being and doing on western derived project management methodologies

v. Utilize a research methodology to bridge normative and descriptive research divides to optimize organizational capacity to learn and change

During the 'finding out' stages of the research and as the social inquiry process progressed, it became obvious that a number of the above aims were most relevant to the exploratory phase of the cultural and logic based streams of investigation in the attempt to unravel the problem situation at hand. In turn, clearer more specific research aims evolved:

\section{Aim 1:}

To explore the significance of socio-cultural context, socio-technical infrastructure and human capital elements relevant to managing projects for effective organizational change

\section{Aim 2:}

To develop a project management technique or strategy which can:

a) accommodate culturally disparate human capital to,

b) facilitate communicative action and knowledge transfer to,

c) promote sense-making for purposeful activity and improved organizational capability

\subsection{Conceptual and philosophical framework}

A research conceptual and philosophical framework has been defined for the research in this dissertation, as is detailed in sections 2.2 and 3.4, and represented pictorially in Figure 4. 
As has been suggested by Cicmil and Hodgson (2006), there is a "...need to explore how the relationships between individuals and collectivities are being constituted and reproduced in the context of project management, and how asymmetrical power relations create and sustain the social reality of projects" (p.13). The assumptions underpinning the defined philosophical framework support the concept of inherent interconnected relationships that exist in project practice, noting projects as social process and as human activity systems in a non-linear state of flux and messiness. Whilst postmodernism influenced thinking in relation to focussing on the importance of individual experience and complexity in human existence as opposed to models of predictability and control, it is enactivism as a theory of cognition that dominates the thinking framework for this dissertation. The usefulness of postmodern thinking relates particularly to exploring the 'knowing-how' of players involved in situations and shares a number of assumptions with socio-ecological approaches that assume an interconnected and embodied nature of human cognition.

Conceptually, the perceived nature and structure of the world in this dissertation focuses on emergent change, evolving processes, transformation and adaptation as reflected in postmodern thinking underpinning a becoming ontology (Chia 1995); and the resilience perspective of complex adaptive systems portrayed in socio-ecological analyses (Folke 2006). The nature of knowledge is then perceived as enacted cognition, culturally situated and emergent through reflective learning, as defined by a pragmatic epistemology underpinning the concept of projects and project organisations in a state of 'being' evolving through enactment to a state of 'becoming' (Lineham and Kavanagh 2006). The critical nature of project practice and the broadening of the project management research theoretical base, supports the notion of projects as socially complex, involving discursive sense-making, socio-cultural disparities and ambiguities in language and communication (CookeDavies, Cicmil et al. 2007).

\subsection{Methodology}

Soft Systems Methodology (SSM) was selected as the action research approach to the social inquiry into what was perceived as a problematical situation (see section 3.6). SSM acts as a qualitative tool to map processes, investigate the problem and define solutions to make improvements to a situation, through facilitating learning for transformational change. SSM is ideal for messy, illdefined problems, where the desirability and feasibility of change evolves through the structured exploration into defining action for improvement (Checkland and Scholes 1990; Checkland and Poulter 2006). Such a methodology complements the ontological and epistemological assumptions framing this research dissertation, as it facilitates a shift away from the more normative, positivist approaches to project management, to support a project actuality that assumes the existence of 
"...complex social processes that go on at various levels of project working" (Cicmil, Williams et al. 2006 p.675).

\subsection{Scope of the research study}

Traditional organizational theory has inadequately explained the significance of the human element in knowledge management and project business practice and has failed to acknowledge the embedded contextual nature of human existence, the significance of power relationships and the manner in which culture affects every day organizational operations (Flyvbjerg 2001; Kakabadse, Kouzmin et al. 2001; Abernathy 2004; Clegg 2006; Marshall 2006). Organizations today exist within an increasingly complex global era, where knowledge as an emergent resource is considered pivotal to organizational survival and change is the constant (Tsoukas and Chia 2002). This research dissertation has aimed to explore the relationship between knowledge, power and language, emphasizing the imperative for those involved in project management theory and practice to acknowledge the centrality of the human element and its relationship to the actuality of projects.

This research dissertation has defined a conceptual framework to guide thinking away from the Weberian modernist rationalist view of an organization as a bureaucratic logical system, predictable in nature and as reflecting social life (Weick 1978; Cooper and Burrell 1988; Chia 1995; Townley 2002), to incorporate postmodern and enactivist discourse to view the project organization as an extension of social life, embedded in a cultural context which is complex, ambiguous and autonomous (Varela 1979; Cooper and Burrell 1988; Rosenau 1991; Tyler 2005). As we move towards a future which seems more complex and 'unknown' than decades ago, where now inconsistencies, uncertainties and multiple dilemmas are the norm, there is an imperative to facilitate sense-making within the project organization through acknowledging and strategically managing competing narratives and disparate groups of people (Barnett 2004; Boddy and Paton 2004; Cooke-Davies, Cicmil et al. 2007).

'Complexity' in project practice has traditionally focussed on the technical and structural elements of the project entity (Williams 1999; Sommer and Loch 2004), giving limited attention to projects as social process where complexity results from disparate social and human interconnections (Winter, Smith et al. 2006; Cooke-Davies, Cicmil et al. 2007). In complex project environments where there is an absence of shared history and culture, and power is socio-culturally derived, intra organizational and project sense-making becomes difficult due to the challenges arising when trying to find some sort of mutual ground for communicative action. 'Shared' understandings, despite apparent objective agreement as expressed through consensual dialogue, cannot be assumed when situations are complex and the cultural underpinnings on which language is based, differ greatly (Freeman 
1999; Derrida in Sikka 2008). The emergence and assimilation of knowledge is intertwined with language, sense-making and human dialogue (Szulanski 1996). The reality that 'being' in a world assumes an embedded nature of knowledge which cannot be separated from a context of meaning (Bhatt 2001; Boreham and Morgan 2004; Clegg 2006), benefits from 'soft' systems thinking where social theory of the organization views learning as enacted to facilitate an ontological shift from 'being' to an active state of 'becoming' (Tsoukas and Chia 2002; Cavaleri 2004; Lineham and Kavanagh 2006).

This research dissertation was implemented in a socio-cultural environment that was complex by the culturally disparate nature of an expatriate workforce. However, the additional 'complexity' that permeated all aspects of daily life, was the reality of predominantly western expatriates living and working within Arabic systems of tradition and governance. The premise that "... human tribalism drives behavior..." (Hilder 2004 p. 632) is a factor that cannot be dismissed in exploring project practice, particularly when relational power is typical of the social inequalities existing. Power as embedded in context, yet emergent, is of particular significance in relation to the tribal history of the Arab world and the sense of group consciousness (assabiyah) or collective mind which defines a particular social group (Khaldun 2005). The reality of the political dynamics which became apparent in the scope of this research (as discussed in Chapter 5), given the history of the UAE and the nature of Bedouin society, is noted as contributing to the complexity of the project environment due to multiple layers of power, history and tradition that impact on today's business practice.

The ontological 'becoming' nature of projects which have been portrayed as " ...emergent outcomes of disparate ambiguous, political practices...' (Lineham and Kavanagh 2006 p.55), reinforce the dynamic nature of projects as social process and the integral nature and relevance of project language to doing business within a global economy (Chia 1995; Nonaka and Takeuchi 1995). Exploring the problematic situation encountered in a culturally diverse socio-cultural environment as described in this dissertation, provided the opportunity to unravel some of the complexities in human interrelations that should be considered and strategically managed for successful project and organizational outcomes.

\subsection{Structure of dissertation}

This dissertation comprises five chapters. The first gives an introduction and overview of the research project, conceptual framework, socio-cultural context and scope of the research project.

The second chapter is a detailed literature review that is subdivided into four main sections, the first of which details the philosophical and conceptual underpinnings of the research as defined as the research framework. The other three sections encompass the topic areas of knowledge and learning 
for change, the changing nature of organisations and the significance of the complexity in context. Within the three latter sections, subsections have been created to explore and discuss relevant factors to the inquiry, including consideration of consciousness, cognition and sensemaking in organizations and the pragmatism of knowledge The cognitive nature of the knowledge-based organisation precedes discussions on the project organisation, organizational capacity to learn and the interconnect between quality and the effective organisation. Discussions throughout the literature review are guided by the thinking framework of enactivism and postmodernism, and are drawn from the project assumptions as presented in the conceptual framework. The centrality of the human element in organizational analyses and project implementation, together with acknowledging the interrelation and interconnectedness of all elements within a project environment, underpin discussions throughout the literature review. In particular, the final section focuses on the biological nature of social complexity, human embeddedness and the influence of language, the realities of culture and religion, power and politics to project actuality and the complex nature of projects as human activity systems.

The third chapter is the Methodology chapter, which introduces the research problem and then details the choice of paradigm and conceptual framework guiding the research. The rationale for selecting Soft Systems Methodology as the research approach to explore the problematical situation is discussed, followed by details of the iterative action learning cycles which ensued. The chapter concludes with two other sections discussing issues of rigor and the ethical considerations for the research.

The fourth chapter presents the research context in more detail and introduces the problematical situation. Four learning Cycles in Action are then described, utilising rich pictures to diagrammatically portray the evolving situation and the desirable and feasible changes identified to bring about improvement.

The fifth and fifth chapter provides a discussion on all the main issues arising from the data, drawing upon the literature review to further expand upon the theoretical base of project management. Limitations to the research are described, with recommendations as relevant to project management theory and practice preceding a final conclusion section.

\subsection{Chapter summary}

This chapter introduces the research as described in this dissertation, providing an overview of the DPM program for which this dissertation was completed. The research context and the problematical situation are presented in summary, with details of a defined conceptual and philosophical framework developed to guide thinking encompassing the research into a problematic situation as 
related to project management. The scope of the research study is summarised, with the research aiming to fill a gap in the project management and organizational theory literature, through exploring the actuality of projects as social process to move beyond the traditional models of projects as instrumental process. The Chapter concludes with an overview of the dissertation structure. 


\section{Chapter 2 Literature Review}

\subsection{Introduction}

Project management practice has tended to draw on traditional organizational analyses, decontextualising project work from the socio-cultural context to explain the perceived realities of business by projects. Human behaviour has been portrayed in rational terms (Weber 1978; Cooper and Burrell 1988; Chia 1995; Flyvbjerg 2001; Checkland and Poulter 2006; Pollack 2007), creating a dis-connect between human existence and action. This has tended to negate socio-cultural contextual factors which are involved in dynamic knowledge flows between individuals embedded in organizations involved in project work (Söderlund 2004a). Projects and the management of projects, whilst increasingly important to the ways of doing business today, have tended to look to existing organizational theory to explain practice, rather than exploring alternative conceptual frameworks which may better fit emerging and unpredictable elements in project environments (Söderlund 2004a; Cicmil, Williams et al. 2006; Winter, Smith et al. 2006). Given the complex and ambiguous nature of today's work environment, it seems timely that there appears to be a shift in thinking from a 'hard systems' paradigm of predictability and control (Winter and Checkland 2003; Atkinson, Crawford et al. 2006; Crawford, Pollack et al. 2006; Maylor, Brady et al. 2006), to one which considers the nature of projects and project management in a 'softer' emerging research light. Calls to expand the theoretical and conceptual knowledge base of project management per se have been made (Cicmil, Williams et al. 2006; Winter, Smith et al. 2006; Turner 2006a; Turner 2006b; Turner 2006c). With there being an increasing recognition of the need to consider human elements and social processes involved in project practice; “... it is people who deliver successful projects, not methods and tools, and it is people's ability to engage intelligently with the complexity of projects, that is central to the successful management of projects" (Winter, Smith et al. 2006 p. 646) .

The research described by this dissertation acknowledges the centrality and embedded nature of human agency to affect a shift in project theory for practice from the instrumental linear image of project functionality to one of social process. A conceptual framework embracing research, philosophical and methodological elements has been defined to explore the problematical situation initiating this enquiry (Figure 4). Complementary paradigms have been identified to guide 'thinking' through the investigative process, with the notion of movement, action and flow considered integral to projects as systems of human activity. The entwined nature of relationships between individuals and groups embedded in socio-political contexts is seen as integral to the emergence of 'different forms of knowing' for action and organizational transformation (Coghlan 2007b). 
Learning as embodied cognition is a continual process that is non-static, emerging from interconnectedness and engagement with others in a pluralistic world of ambiguities and discursive meanings (Vygotsky 1978; Argyris 1999; Wenger 1999; Engeström 2001; Healy 2003; Barnett 2004). Knowledge emerges and meaning arises 'through and from' sensemaking activities, with learning being the result of reflection on experience, dialogue, interpretation and application (Kolb 1984; Rowley 2003; Coghlan 2007a). Organizational learning for improved performance and outcomes may be seen as resulting in part from knowledge flow within and between interconnecting human elements and in part from the manner in which the flow is managed and facilitated (Nonaka 1994; Crossan, Lane et al. 1999). But given the embedded nature of cognition being enacted as a continual social process whereby knowledge is not so easily reified, it is in actuality the intersubjective nature of human existence within a project organization which needs to be 'managed' to connect knowing and doing. Acknowledging the social process of projects thus requires attention to the mix of social agendas and stakeholder relations which need to be identified and wisely managed to ensure that actors capacity to reflect and act for emergent change is facilitated (Winter, Smith et al. 2006). 


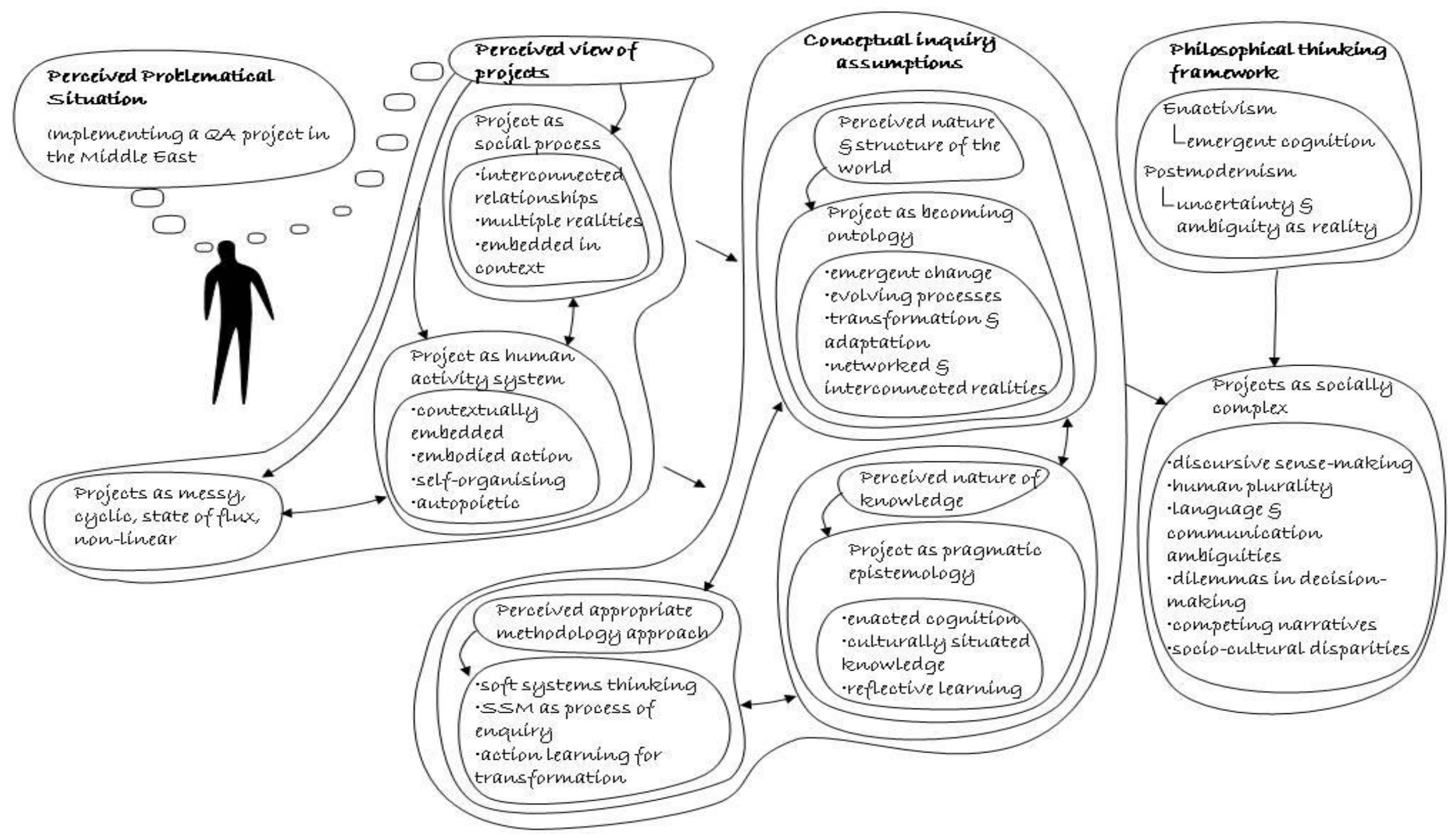

Figure 4 Research conceptual framework 
Complexity in relation to project management is noted as a key factor particularly at the front-end of project implementation (Turner 1999; Williams 2002). However, the nature of 'complexity' described in the literature reflects the origins of project management in the construction and IT industries, where structural and functional complexities, ambiguities arising from project goals and methods, multiplicity in technical elements or detailed information processing are the order of the day (Turner 1999; Williams 2002; Campos and Sanchez 2003; Söderlund 2004a; Ivory, Alderman et al. 2006). Alternatively, complexity in relation to organizational systems, tends to emphasise knowledge management processes, structural factors related to organizational learning and organizational functionality (Cilliers 2000; Engeström 2001; van Eijnatten 2004).

What is missing from the project management literature is detailed exploration into the implications for practice when 'complexity' is socio-culturally derived due to key actors originating from diverse cultural and social backgrounds. Although the significance of competing narratives in project contexts has been raised by some (Boddy and Paton 2004) and the importance of project sensemaking activities through shared discourse by others (Ivory, Alderman et al. 2006), the predominant issues of cultural differentiation in project management appear to be found in research related to objectified knowledge management and transfer in large scale multinational and cross-cultural projects (Barkema, Shenkar et al. 1997; De Long and Fahey 2000; Bhagat, Kedia et al. 2002; Glisby and Holden 2003; Javidan, Stahl et al. 2005; Marsella 2005; Javidan, Dorfman et al. 2006; Chuah, Hoffmann et al. 2007). Project complexity arising from a cultural mix of actors enactively working together within the one organization however, has received limited attention (Cooke-Davies, Cicmil et al. 2007). The tendency to sideline the essential nature of human agency through portraying 'knowledge' as an objectifiable resource that can be captured, transferred or shared, has limited the exploration of knowledge as “... a culturally situated and ongoing social activity..." (Orlikowski 2002 p.251).

The impact of such a 'complexity of context' arising socially from a diverse mix of cultures, religions and discursive languages, and the potential these elements have to exacerbate relational power and political factors in a project management environment, have received limited investigation (Watson, Kumar et al. 1993; Szulanski 1996; De Long and Fahey 2000; Gupta and Govindarajan 2000; Kakabadse, Kouzmin et al. 2001; Glisby and Holden 2003; Clegg 2006; Marshall 2006). The research as described in this dissertation is informed by Maturana and Varela's (1980) concept of ${ }^{1}$ autopoiesis and enacted cognition, noting the embedded nature but active process of 'knowing' through reflective practice where autonomy takes precedence over control. The research in this dissertation originates from difficulties encountered when attempting to implement and manage what became a 'complex project' due to an organisation's diverse socio-cultural human capital mix where language

\footnotetext{
${ }^{1}$ See glossary of terms
} 
differences reflected variations in thinking and doing. This was compounded by the transient nature of expatriate worker employment and the dichotomy between the organisation's culturally derived system of governance and the operational expectations of expatriate senior management. Application of traditional instrumental models of project management fail to accommodate for ambiguities and discursive realities that exist in socially complex project management settings. It is proposed that through viewing projects as human activity systems involving social process, an expansion to the theoretical and conceptual base of project management will be facilitated. This has the potential to improve and add value to project performance through shifting thinking about knowledge as being objectively definable or processually dispersed (Tsoukas 1996; Orlikowski 2002), to knowledge as enacted, with consciousness, cognition and sensemaking inextricably linked as a social activity.

The realities of social context interconnect with the realities of learning and project complexity. It is suggested that an increase in the culturally complex nature of a social context will directly correlate with uncertainties and ambiguities in project management settings. To accommodate for uncertainty, post-modern thinking, where certainty is not assumed to exist (Rosenau 1991), is a useful way of reflecting on the workings of such project contexts. Uncertainty underpins an ontological shift from the static concept of 'being' in a world, to an ontology of 'becoming' where the emphasis is on "... transient, ephemeral and emergent..." realities (Chia 1995 p.579). Such emerging qualities may reflect an organization's capability to not only respond to changing business demands through utilizing projects as process, but may also be evident in the organisation's capacity to actively create a 'becoming' identity to shape an environment to gain competitive advantage (Nonaka and Takeuchi 1995; Crossan, Lane et al. 1999; Jerez-Gomez, Cespedes-Lorente et al. 2005). It is the enactment of knowing in socially complex project environments that warrants further investigation. Project praxis results from the actuality of an interconnect between action and reflection, supporting the notion of projects as knowledge generating cognitive systems contextually embedded.

This chapter is structured to provide an overview of the research, philosophical and conceptual framework underpinning intellectual thought, followed by sections giving consideration to knowledge and learning for change, the changing nature of organisations and the elements and realities of contextual complexity as relevant to project actuality.

\subsection{Research philosophical \& conceptual framework}

The $20^{\text {th }}$ century has seen the rise of a 'new' knowledge era, where the value inherent in the human capital element of the organization is recognized as integral to sustainability (Moingeon 1996; Engeström 2001; Walker 2004; Cavaleri, Seivert et al. 2005; Davis 2005; Kodama 2006). This has 
created an imperative to move beyond the Weberian modernist rationalist view of bureaucratic organizational life whose functionality is beyond human control (Cooper and Burrell 1988), to acknowledge organizations as systems which may be organizationally closed, yet structurally open and autonomous, comprising individuals whom contribute to constructing a world through interpretation for improved sense-making (Varela 1979; Cooper and Burrell 1988; Weick 2001; Ivory, Alderman et al. 2006). The changing nature of organizations has evidenced a shift in managing business from relying on predictable structural and functional aspects of the work environment, to using projects as a conduit for successful outcomes through facilitating responsive, flexible and proactive ways of doing (Packendorff 1995; Turner 1999; Söderlund 2004a; Cicmil and Hodgson 2006; Crawford, Pollack et al. 2006). Pivotal to this responsiveness is the capability of an organization to embrace new ways of thinking so that creative, innovative solutions can be found to facilitate necessary change. Projects, as cognitive social systems have the potential to function with a degree of autonomy to move beyond organizational control, through promoting internal and external structurally coupled relationships with other autonomous systems in consensual domains. Such interrelationships are necessary to sense perturbations in the environment, which in turn will necessitate interpretation and knowledge creation for making changes for survival.

An integrated research and philosophical conceptual framework has been defined to portray the presupposed embedded and enacted nature of knowledge, which is continually being reconstituted by actors in a project management setting (Figure 4). The concept of 'being' in time may be traced to the Heideggarian phenomenological tradition of the nature of consciousness. However, the conceptual framework for this research is informed more by the works of Varela, Thompson et al (1991) in relation to cognition as embodied action, and earlier works of Maturana and Varela (1980) where living systems may be seen to be structurally coupled with the environment as autopoietic networks (Capra 1997). Cognition is considered as being central to "... a continual bringing forth of a world through the process of living" (Maturana and Varela 1987 p.174), with knowing being a result of being and doing (Reid 1996). Considering the social nature of projects, it is suggested that projects function as human activity systems that are autopoietic in nature as are other living systems. Organisational sustainability working through projects is dependent upon maintaining a balance of 'being' and an emerging 'becoming' through incorporating reflective learning for continual selfgeneration and self-perpetuation. Interactions within a socio-cultural environment of project actors allow for modifications being made to project structure and function, while preserving the overall project's organizational character for effective outcomes.

Enactivism, as a cognitive theory of the mind facilitates the conceptualization of project management as embodied action. Such thinking supports the complex nature of projects that involve multiple interrelationships, through linking the active components of knowing and doing. As noted by Smith 
(2005) 'embodiment' assumes "...that intelligence emerges in the interaction of an organism with an environment and as a result of sensory-motor activity" (p.279).

Thinking for this research is supported by postmodern discourse, which questions impermeable boundaries between life forms, is suspicious of reason and rationality, seeks meaning in chaos, favours difference over standardization and accommodates a multiplicity in worldviews (Cooper and Burrell 1988; Rosenau 1991; Hasselbladh and Kallinikos 2000; Marsella 2005; Sikka 2008). Such thinking complements the enactivist theory of cognition, through contributing to a philosophical conceptual framework that encourages reflection on human existence as an ontology of emergence, therein facilitating sensemaking in an era of confusion and uncertainty (Chia 1995; Höijer, Lidskog et al. 2006; Ivory, Alderman et al. 2006; Lineham and Kavanagh 2006). Philosophically, postmodernism has been influenced by existentialism, structuralism and phenomenology, and while the thinking helps us appreciate the messy realities of life, enactivism provides a theoretical framework to consider complex systems, particularly in relation to cognition, consciousness and the heterogeneous individual realities of knowledge construction and interpretation. It has been suggested that "...the knowledge of the man who acts and thinks within the world of his daily life is not homogeneous; it is (1) incoherent, (2) only partially clear, and (3) not at all free from contradictions" (Schutz 1967 p.93). It is proposed that it is the interpretations of such ambiguities that are required for sensemaking not only in organizations, projects or environments per se, but in order to facilitate an understanding in and between individuals intersubjectively connected in a world of complexity.

Projects as the 'new' and emerging way of doing business in this increasingly complex world, have traditionally been designed, implemented and analysed in the same vein as organizational theory, with thinking being cloaked in rationalism, internal control, bureaucratic logic and metanarrative discourse (Söderlund 2004a; Pollack 2007). Complexity - whether the organization, the project or the environment, has predominantly been defined in terms of 'structural complexity and uncertainty' (Ivory, Alderman et al. 2006), with there being little investigation into project complexity arising from the effects of socio-culturally derived multiple realities upon project process and outcomes. The project has instead, tended to be reified as a definable object of equal relevance and applicability despite the context (Chia 1995; Söderlund 2004a; Hodgson and Cicmil 2006). This is not unexpected if thinking has been framed in positivist modernist analysis, however, such practice is inadequate in unearthing the emerging ambiguities which exist in organization functionality and fail to address the realities being faced in project management settings in today's era of uncertainty (Barnett 2004; Höijer, Lidskog et al. 2006). Thus, a project 'becoming' ontology which reinforces the concept of emergence and shifts thinking beyond 'being' in the world, is supported by a pragmatic epistemology, where active, reflective learning facilitates knowledge creation and transfer. Conceptualizing projects as social process involving complex, non-linear, multiple realities better 
represents the messiness and context dependency of project management and the realities of projects as human activity systems.

The lack of empirical debate on complexity arising from a human plurality of cross-cultural differences creating a collage of multiple realities and interpretations on meaning of events warrants exploration. Sensemaking in situations of human plurality is at the core of communicative dialogue and when individuals from different social worlds and collective minds gather, a unified language of comprehension to reach a consensual domain is evasive. Gephart (1993) refers to sensemaking as a process from which the "...the intersubjective or cultural world is constructed or produced" (p. 1469). But when the realities of members from different cultural worlds inter-connect, it is suggested that although there may be some degree of assimilation in meaning, actual consensus of meaning due to the uniqueness of world views, is not possible. It is this 'difference' which according to Cooper and Burrell (1988) is the key to understanding postmodern discourse and underpins the assumption that unity is difference (Derrida 1992), consensus is elusive (Lyotard 1984), and "...different interpretations of complex settings are most likely to coexist" (De Paula 2005 p. 1). The enactivist view of complex systems which, as for projects, may reside in a complex setting, is that they are selfreferential, undergoing constant emergent structural change involving cognitive processes, while preserving the autopoietic nature of the project system (Reid 1996). Projects as cognitive systems or intelligent systems therefore need to be structurally coupled with other project entities, the broader organizational entity and the surrounding environment, for interaction and mutual learning through intersubjective relationships to take place (Franklin 1997; Riegler 2002).

Giving consideration to the dilemmas and tensions which inevitably arise when cultural multiplicity of human worlds collide in a project management setting where projects function as human activity systems, the challenges faced need constructive management for effective outcomes. As Chia (1995) states, "The shock and strangeness of confronting a different cultural order reveals the limitations of a particular thought style when we reach outside the familiar conventions and ordering codes that define our particular view of the world" (p.583). This research described in this dissertation acknowledges the postmodern assumption that arise from Nietzsche's concept of 'genealogy' which states that 'difference' is the driving force behind change and transformation (Emden 2005), with dissent and conflict having potential benefits if mutual agreement can be achieved. It is proposed that the challenge for project managers in organizations attempting to reconcile learning capability with knowledge transfer systems, is to facilitate consensus action despite discursive interpretations of the problem situation at hand, through focusing on aspects of learning rather than control (Thomas and Tjader 2000).

To adequately consider the human element in project implementation, the first step is to recognize projects as social process. The centrality of consciousness and cognition in relation to understanding, 
interpretation and learning then logically follows. The philosophical underpinnings of enactivism assume that we actively experience a social world intersubjectively with others while cognitively making sense of a world in which we are embedded (Schutz 1967). Sensemaking is facilitated by being 'structurally coupled' with other individuals and the environment, with the project acting as an autopoietic system which is organizationally closed but structurally open between individual entities and the surrounding environment. This structural coupling is defined as "...a history of recurrent interactions leading to the structural congruence between two or more systems" (Maturana and Varela 1998), and is crucial in determining the project entity.

The research conceptual framework is in turn supported by the methodological framework of Soft Systems Methodology (SSM), which accommodates investigations into ill-defined, 'fuzzy' situations where uncertainties and real-life human problems exist. SSM potentially facilitates hermeneutical enquiry into seeking individual meaning and understanding of a problematic situation through sensemaking and purposeful activity. Schutz's (1964) phenomenological constructivist models of human action and socially derived knowledge assume contradictions exist in and between human realities. However, for purposeful enquiry where the researcher is embedded as an active participant or 'insider' (Coghlan 2007b) in the problem situation, SSM more ably accommodates the realities of interconnected consciousness through action research. Inquiry into the problem situation presented in this research dissertation integrates three levels of research inquiry. Firstly there is investigation of the problem through individual reflection, then secondly, sharing these findings with others having mutual concerns and then thirdly, presenting the findings and action recommendations from the exploratory process to the objective reader of this dissertation. Any inquiry that incorporates perceptions of a researcher in context into situational reporting needs to ensure transparency, and such a 3-tiered approach has been noted as improving the integrity of the research (Coghlan 2007a).

The integrated research and philosophical framework presented primarily explores the active and complex human factors involved in projects as social process (Winter, Smith et al. 2006), noting that projects are emerging entities which can be considered as autopoietic human activity systems created from the generation of knowledge arising from intersubjective relationships of actors (Cicmil, Williams et al. 2006). Philosophically, a becoming ontology of projects is constituted by actions of interrelated actors, and a pragmatic epistemology fosters knowledge co-production through active, reflective learning and sensemaking. Such a conceptual framework supports praxis-based theory where projects are seen as complex, non-linear and involving multiple realities (Cicmil, Williams et al. 2006). This reflects a more contemporary way of thinking about projects as social process, moving beyond instrumentality to foster a greater appreciation of the multitude of factors involved in developing reflective practitioners for socio-culturally complex project contexts (Winter, Smith et al. 2006). 


\subsection{Knowledge \& learning for change}

\subsubsection{Consciousness and cognition for sensemaking}

The conceptual framework defined for the research described in this dissertation (Figure 4) presupposes an embedded and interwoven nature of reflection and action for knowledge creation and flow in a project environment. An 'ontology of being' assumes that 'knowing is doing', with an emergent 'ontology of becoming', made possible through reflective practice and action. Cognition is seen as the social expression of interconnected individual states of consciousness that merge in and through the social reality of projects, where the actuality of projects involves recognition that they exist as knowledge-generating human cognitive systems. It is thought that a balance is necessary in project management between promoting a state of 'being' while facilitating an emergent entity of 'becoming' which is made possible through pragmatic learning from reflective practice and interpretation. Language, as the means of communication and facilitator of social interrelatedness within projects, is the medium instrumental in ensuring the system develops inherent capabilities to mutually create and adapt to local conditions. Yet, words are only one way of explaining particular perceptions of a world as they "... can not undo the fact that we are surrounded by it" (Berger 1977 p.7). Collective consciousness therefore emerges through discourse, which supports embedded knowledge creation through sensemaking. This facilitates the balance necessary for project existence with the capacity to act being determined by the degree of autonomy a project entity of assimilated human realities is privileged. Unsatisfactory project outcomes may be directly attributable to a failure in facilitating sensemaking activities arising from complex human plurality and discursive discourse.

The following section considers the evolving philosophical nature of knowledge in relation to consciousness, cognition and sensemaking, to precede discussions on the relevance of pragmatic knowledge as actionable knowledge in socially complex project environments.

Knowledge has traditionally been perceived as an objective and measurable entity that can be controlled, codified, stored and transmitted (Nonaka and Takeuchi 1995; Nahapiet 1998; Teece 1998; Zack 1999; Fischer and Ostwald 2001; Kakabadse, Kouzmin et al. 2001; Healy 2003; Kodama 2005). Knowledge, rather than being emergent as described in the introductory section 2.1, has philosophical origins of being 'justifiable' attributed to Plato's (b. circa 426 BC) concept of 'complete

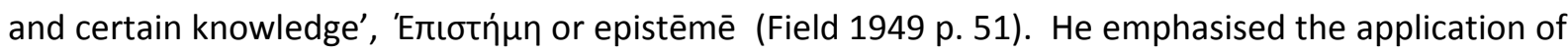
reason for knowledge acquisition, with his conceptualization of 'Form' as the ultimate expression of an object's essence, with sense-perception and memory only playing a role in knowledge development through triggering internal pre-existing metaphysical or innate knowledge (Field 1949). 
The belief that reasoning and pre-existing principles act as the source of ultimate truth and thus knowledge about the world, has remained as underpinnings to the historical scientific intellectual rationalism and cognitive realities of the social sciences (Healy 2003). These in turn have influenced approaches to managing the realities of social and business life. Yet given the increasingly complex nature of the world around us, where uncertainties and dynamic change dominate, it seems that in relation to project management practice traditional rationalistic approaches to conceptualization of knowledge are not only inadequate, but may actually be instrumental in project failure (Ivory, Alderman et al. 2006). As noted by Frans van Eijnatten (2004) "Working in the $21^{\text {st }}$ century is different from all that has come before. In the midst of complexity people increasingly are facing inconsistencies, contradictory demands, and dilemmas in decision-making" (p.430). Knowledge creation can no longer be considered a result of linear, intellectual processes, rather it is suggested that knowledge is more accurately portrayed as an emergent property of interconnecting sensory and cognitive elements that facilitate interpretation, responsiveness, adaptation and transformation.

The role of individual perceptions in knowledge development was noted some 2000 years ago by Aristotle who suggested that sensory experience facilitates understanding of the world through making impressions on an 'empty mind' rather than one with pre-programmed consciousness (Thomson 1955). However, it was not until John Locke's (1 $7^{\text {th }}$ century AD) conceptualization of the human mind as a 'blank slate' as being receptive to creating knowledge through senses, that a more autonomous theory of knowledge developed (Limone and Bastias 2006). This primarily facilitated a shift in thinking away from rational cognition to an epistemology primarily sourced by sensory and experiential means, but the separation between the 'real world' and self remained (Jashapara 2007). This separation of sensory perception from intellectual enquiry, and the subjective (as opposed to collective) nature of knowledge acquisition, has continued to dominate positivist western and eastern philosophical thought (Hasselbladh and Kallinikos 2000; Macgill and Siu 2005; Jashapara 2007; Pollack 2007; Sikka 2008). Furthermore, it has been instrumental in perpetuating mechanistic linear models of management which tend to rely on outdated premises of predictability of cause and effect (Glass 1996). Knowledge as emergent through reflection and action of interconnected realities, where sensory perception integrates with rationality, memory and interpretation, moves beyond the cognitive dualism as portrayed in the expression, 'Cogito, ergo sum' - "I think, therefore I am" of René Descartes ( 1644 in Veitch 2002). Justifiable knowledge as an absolute truth seemingly fails to accommodate for the collective and embedded nature of consciousness, in a world where increasing ambiguities, change and diversity are the norm (Barnett 2004; Höijer, Lidskog et al. 2006). Uncertainties in knowledge about 'being in a world' reflect the social messiness that exists and the crucial nature of culture and context in relation to cognition and sensemaking (Höijer, Lidskog et al. 2006). It seems apparent that "... descriptive-analytical reason ... cannot deliver certainty in a way that allows far-reaching conclusions about future developments to be drawn" (Höijer, Lidskog et al. 
2006 p.364). An 'objective ontology of reality' forms the operational foundations of rationality (Maturana 1988), negating the role of emotions and sensory perceptions in cognition. Such an approach is considered inadequate when viewing knowledge in the context of project praxis.

Although the beginnings of an interconnect between senses and cognition became apparent in Immanuel Kant's “...transcendental idealism” (late $18^{\text {th }}$ century), where the mind was considered as having receptive and conceptual capacities, knowledge beyond the empirical was still not considered possible, as perceptions were thought of as not necessarily real. The gap between the rationalism of Descartes and the empiricism of Locke began to narrow (Nonaka and Takeuchi 1995) in the time of Kant. But a real shift from mind-body dualism was not apparent until the time of Husserl's (1931) phenomenological theory, where knowledge as 'pure consciousness' presented 'Being' as absolute, beyond empirical subjectivity and 'cogito' as "... pure intuition and as an act of pure consciousness" (Scott 2003 p.3). These concepts which in turn formed the basis of Heidegger's (1927) philosophy of 'being in the world' (Dasein), emphasize the temporal nature of existence where a phenomenological ontology of 'being' takes precedence over a phenomenology of consciousness. Thus as thinking began to move away from knowledge acquisition being constructed from subjective experience of a world to embodied intelligence seen as emerging “... in the interaction of an organism with an environment and as a result of sensory-motor activity" (Smith 2005 p. 279), less emphasis was placed on the logical behaviourist notion of knowledge creation, to that of knowledge construction being experientially active but constrained by how an individual performs in an objective external world (Maturana and Varela 1980; von Glasersfeld 1990). The aligning of autopoietic systems with cognition then further shifts the learning theory foci from knowledge acquisition being constructed from subjective experience of a world, to having the learner embedded in context. Cognition is thus enacted from the human entity relating to its surroundings in an irreducible and indistinguishable manner (Varela 1987).

The historical traditions of reifying 'knowledge' as a definable entity whose source or trigger is externally derived has permeated a trajectory of epistemological and ontological history. It may be seen in the justifiable knowledge as the 'Absolute truth' of Platonism (Field 1949); in early Islamic philosophy of Abū 'Alī al-Husayn ibn 'Abd Allāh ibn Sīnā's (c980 BC) where 'self' is independent of physical substance (El-Bizri 2003); in Ibn Khuldun's writing (14 ${ }^{\text {th }}$ Century AD) on the power of thinking as influential on the soul (Khaldun 2005); in the mind/body split of the Descartian tradition (Veitch 2002); Ryle's 'knowing what' and 'knowing how' (1949); Polanyi's (1966) 'tacit knowing' which becomes explicit in consciousness through social interaction; and in the tacit/explicit distinction of Nonaka and Takeuchi's (1995) 'socialization, externalization, combination internalization' (SECI) organizational learning knowledge process spiral . 
However, despite attempts made to connect the separatist notion of the human entity as relating to the world through portraying knowledge on a continuum (Ryle 1949) or as a social learning process (Vygotsky 1986; Nonaka and Takeuchi 1995), there has remained an inadequacy in the innate/tacit, intermental/intramental or tacit/explicit knowledge conceptualizations due to an ontological vagueness of the 'unknown' (Jashapara 2007). The positivist tendency to continue to objectify knowledge into mutually independent entities perpetuates an instrumentality and logic to processes that are complex and variable in enactment. Such thinking fails to adequately cater for the realities of emerging project praxis in a world that is increasing in complexity.

The research described in this dissertation has thus defined a conceptual framework informed by enactivism, to provide a more wholistic perspective of consciousness where "...consciousness is completely material and irreducibly mental at the same time..." (Searle 1999 p.69), to shift thinking from the dualistic nature of a mind-body split in knowledge creation, to one of unity. The rationality of hard-systems thinking found in current 'taxonomic' (Tsoukas 1996) perspectives of organizational knowledge and positivist views of project practice, negate the realities of complexities found in a project environment. In contrast to the dominant rationalist and positivist approaches that have influenced traditional and historical epistemology, an enactivist conceptual framework which notes the embedded nature of cognition where boundaries of knowledge are blurred or indefinable, more accurately portrays project actuality. Projects as human activity systems, with enacted cognition resulting from human interchange and interconnected realities, underpins a pragmatic epistemology of learning through collective action. Projects as social process involve a collective consciousness through facilitating dialogue and interaction to cater for the plurality and uncertainties facing us today. The 'act of knowing' may be seen as enacted consciousness, where knowledge is not only a process of active knowing, but emerges epistemologically to create gaps, disturb the world and bring forth a new world of becoming (Barnett 2004; Cavaleri, Seivert et al. 2005) . Pragmatism moves the conceptualization of knowledge beyond Absolutist theories of an ultimate, justifiable truth and disputes the Skeptics position that we can never know the absolute truth, by emphasizing the human ability to make choices about deciding what knowledge is justifiable and what is not (Meyers 2005).

Consciousness therefore, arising from enacted cognition, may be seen as a biological phenomena (Maturana and Varela 1980; Searle 1999) where the mind/body duality is negated (Jashapara 2007). As a theory of cognition, enactivism is thus pivotal to the conceptual framework of this research, as it acknowledges the clear link between reflection and action for knowledge creation and flow. According to Reid (1996) enactivist theory is informed by the works of Merleau-Ponty (1962), Wittgenstein (1953), Bateson (1987) and Maturana and Varela (1998) with the central concepts of embodied cognition being attributed to Varela, Thompson et al (1991). The embodied nature of learning, where human cognition remains inseparable from the physical realm, encompasses all 
patterns of learning, including those arising from complex cultural systems which are self-referential and self-perpetuating (Reid 1996). Projects as a social system may be considered autopoietic, in as much as they remain in existence through ensuring a constancy in organization while adapting their structure through 'reading' the environment, reflecting on past experiences and acting whilst maintaining a degree of autonomy from the 'main organisation'. Although the autopoietic nature of social systems has been questioned (Hejl 1980; Kay 2001), with even Maturana and Varela (1980) suggesting autopoiesis is not directly transferable to social systems, for purposes of this research the central concepts of autopoietic theory are considered relevant, particularly in relation to the issues pertaining to social interrelatedness for system survival.

Projects as emergent, where emergence may be defined as "... temporary but coherent coming into existence of new forms through ongoing intrinsic processes..." (Smith 2005 p.278), align memory, knowledge and adaptation with 'being, doing and knowing' (Lave and Wenger 1991), to facilitate projects as embodied entities to evolve. Although it has been claimed that this emergence is assumed rather than explored (Hejl 1980; Kay 2001), it is proposed that the centrality of reflective practice for learning, is the critical element integral to the actuality of emergence. The essence of 'being' in a project management environment involves the embedded nature of multiple realities cognitively connected through discourse to create a shift toward a collective consensual consciousness. Project outcomes thus emerge as 'becoming' non-linear entities (Chia 1995 p. 582; Baerveldt and Verheggen 1999; Lineham and Kavanagh 2006) as sensemaking through reflexive practice facilitates the shaping of the future through human action (Giddens 1991).

The nature of social complexity in project practice assumes an inevitability in discursive dialogue due to variances in socio-cultural backgrounds and diversity in ways of being and doing in the world where human actors as observers may exist in ontologically opposing domains (Maturana 1988). Differences in language, where "... the word is more than just an instrument which makes dialogue possible... [rather it comprises ]... two dimensions, reflection and action in such radical interaction that if one is sacrificed - even in part - the other immediately suffers" (Freire 1970 p.60), add to the uncertainties and dilemmas arising from project complexity. If, as stated, we view projects as human activity systems where cognition is emergent and complex and dialogue is the facilitator of communication for connecting the human element, then language becomes the critical factor in knowledge flow (see section 2.5.2 re the influence of language). Whilst retrospection assists in making sense of a situation, there is an assumption that there is a level playing field when it comes to cognitive processes. Yet the complex nature of cognition as an embodied entity reacting to perturbations in the environment (Maturana and Varela 1980; Riegler 2002), involve individuals and groups interpreting situations uniquely according to variances in consciousness, experiences and memory. Maturana (1988) has presented these differences as two contrasting ontological domains 
where observers in a praxis of living may exist in either a transcendental ontology where existence is validated by a universal reified reality or as constitutive ontology where coherence arises through multiple legitimate realities, where "...every statement that an observer makes is valid in some domain or reality, and none is intrinsically false" (Maturana 1988 p.30) (see below)

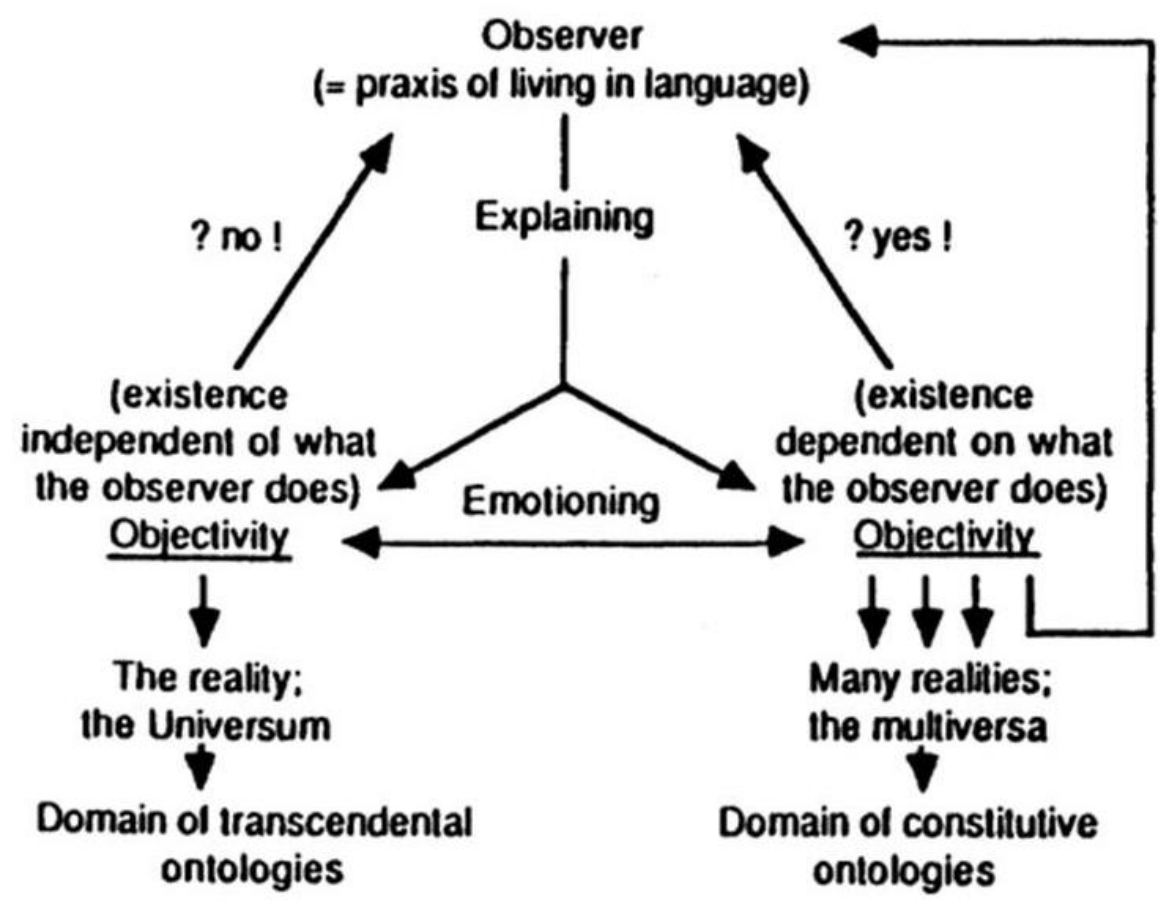

Figure 5 Maturana's reality ontological diagram (1988 p.5)

Projects as a functional entity relying on an element of consensus in action, therefore need to move beyond universalizing knowledge or validating explanations through appealing to a universal reality, to acknowledge the many realities which constitute our living praxis. Catering for the socio-cultural nature of multiple realities is the project management challenge, but it is suggested that through encouraging the difference which is found in human plurality (Healy 2003) and rejecting the idea of an absolute truth, that a closer alignment between the domain of cognition and the domain of action will be facilitated.

Although there has been "... very little detailed analysis available of the social mechanisms that support knowledge sharing, especially across projects ..." (Bresnen, Edelman et al. 2003 p.158), it is argued that social interchange through communicative dialogue reflects the individual parts of a system which can only be appreciated through considering the dynamics of the whole (Capra 1997). Aligning project practice with enactivist theory, assumes individuals are structurally coupled with each other and within a context, acting together and knowing in action. Cognition is therefore inseparable from life (Varela 1987; Reid 1996; Capra 1997) and “...knowledge is part of a dialogue 
between subject (the human world) and object (the physical world)" (Macgill and Siu 2005 p.1107). Thus, projects which are culturally complex will present a multiplicity in ways of knowing and doing as individuals from diverse backgrounds are working together. Facilitating sensemaking whilst encouraging difference and balancing competing narratives in and through situated human plurality (Healy 2003; Boddy and Paton 2004) becomes the challenge for the project manager. The intersubjective nature of human existence assumes a cultural interrelatedness, where culture is a process which facilitates shared interpretations and understandings (Schutz 1967). Sensemaking becomes the process which helps construct this intersubjective or cultural world (Gephart 1993), where the intersubjective world is "...the world known and held in common by some collectivity of persons" (Schegloff 1992 p.1296). However, when the cultural backgrounds of project actors are divergent, then the resultant complexity inevitably causes discursive ways of interpretation, making efforts at 'shared' meaning through 'sensemaking' activities challenging.

The concept of 'sensemaking' is drawn from Schutz's (1967) phenomenological writings, wherein understanding emerges and is constructed from intersubjective interactions of individuals seeking meaning in their life-worlds. Sensemaking in organizations, both conceptually and in reality has been explored in the strategic management and organizational analyses literature (Gephart 1993; Weick 2001; Ivory, Alderman et al. 2006), yet, as noted in Ivory et al (2006) there has been lesser application in project management theory and practice (Thomas and Tjader 2000). This research dissertation aims to explore some of the dynamic realities of knowledge creation where the actuality of projects entails a plurality and cultural complexity in human capital. The 'relationship asymmetries' (Inkpen 1997) and 'contextual asymmetries' (Clark and Geppert 2006) that result from plural cultural mixes in an expatriate working environment, where Western ways of doing have been imported, for example, into an Arabic system of governance, inevitably involve friction and conflict in knowledge transfer and assimilation. Organizational transformation is emergent, but management of socio-political inequalities and knowledge differences are necessary to rebalance relationships of autonomy and control, power and domination. It is suggested that through considering projects as autopoietic human activity systems, that a shift from focusing on individual sensemaking to that of emerging relational knowledge resulting from interconnected realities, will be facilitated despite disparate ways of knowing. Reconceptualising knowledge as embodied cognition within a project which is organizationally closed, yet structurally open and emergent, has the potential to embrace epistemological pluralism which is argued to be of greater relevance to project sensemaking and organizational transformation.

Knowledge creation as active, where 'knowing is doing' for emergent realities is supported by an epistemology where reflective practice and experiential learning underpins the pragmatism of knowledge. "...we discover that our experience is that we find ourselves observing talking or acting, 
and that any explanation or description of what we do is secondary to our experience of finding ourselves in the doing of what we do" (Maturana 1988 p.26). The following sub-section aims to align pragmatic knowledge with embodied cognition, noting the relevance that project realities have in working with socially messy situations.

\subsubsection{The pragmatism of knowledge}

The origins of 'pragmatic knowledge' may be traced to the early philosophical writings of Charles Peirce (1839-1914) who, as the 'founder' of pragmatism, claimed that human beliefs originate from 'habits of action' and 'truth' is defined as inquiry (Frankenberry 1996). Whilst William James (1907) extended the relationship between knowledge and action - 'if an idea works, it is true' - to a theory of truth (1975), it was Peirce and his theory of meaning through experience, reality and consciousness, who presented pragmatism as a method of clarifying ideas (1868). Given the complex and uncertain nature of today's project environment, this concept of 'truth' based on rational social and cognitive realities tends to simplify knowledge as having a cause and effect relationship, however it does reflect the beginnings of a closer interconnect between experience and conscious thought. John Dewey (1929) also advocated 'learning by doing' linking knowing with action, with his views on morality as being relative to individual experience noted as underpinning the ethical theory of instrumentalism. He extended upon Peirce's concept of clarifying ideas, claiming “... ideas are worthless except as they pass into actions which rearrange and reconstruct in some way, be it little or large, the world in which we live" (Dewey 1929 p.138). Both James and Dewey instigated a radical shift away from the absolute nature of truth and knowledge, by introducing the concept of individual choice in deciding what to believe and thus justify. Their pragmatic epistemological approach to learning moved beyond the Absolutist claims of a finite truth and away from the Skeptics who disputed that a belief could ever be true. They introduced the concept of justifiable beliefs where " ... the idea that we are justified in claiming that something is true if we have enough evidence for it, even if it is still possible for the idea to be wrong" (Meyers 2005 p.3). This reflects a shift to a more flexible approach to the meaning of knowledge, with Boisvert (1998) identifying Dewey as the first postmodernist whose learning theories move beyond the didactic notion of information exchange, to one of learner interconnect and emergent realities.

It is such thinking which is considered more relevant for our current world of flux and change, where knowledge can no longer be viewed as predictable, static and universally reified. However, there is an imperative, as noted by Healy (2003), that the centrality of knowledge per se remains integral to determining future directions, as it "... acts to shape our world, culture, institutions and actions and embroils us in complexes of both people and things, paints a complexity remote from the certainties 
conveyed by representational understandings" (p. 693). He claims that 'epistemological pluralism' enables a reconceptualisation of knowledge, with power relationships being pivotal to knowledge creation, use and dissemination. It is noted that this pluralism has been fuelled by globalization of market economies, application of expansive communicative technologies and ease of human transnational mobility, thus increasing the permeability of nation-state borders and cross-cultural information exchange. These changes rather than facilitating a uniformity or standardization of 'knowing' have instigated disparate ways of understanding and created a demand for greater tolerance for variations in individual and community socio-cultural value systems and ways of knowing and doing. This 'pluralism' of ideals underpins the need to recognise the existence and inherent nature of human multiple realities in which knowledge is created, shared, assimilated and disseminated. Sustainable future project management is dependent upon appreciation and incorporation of these realities into daily business practices utilizing pragmatic leadership to integrate a 'mix of knowledge' (Cavaleri, Seivert et al. 2005) and manage the inevitable power struggles that result from individuals attempting to make sense of their world through justifying their beliefs in the construction of knowledge.

Projects as human activity or social systems are not only able to provide a medium for facilitating the communicative exchange pivotal to knowledge creation, but they may be seen to act as a system of 'co-ordinations of actions in and through language' (Maturana 1988). Pragmatic knowledge is actionable knowledge, where individual belief and choice in deciding what a justifiable truth is, may be seen to provide a more relevant, flexible model of knowledge creation which draws on the situated nature of interconnected realities for an emerging ontology. As Cavaleri, Seivert et al (2005) have noted, "... the pragmatic approach links action learning with knowledge creation by uniting the inner and outer worlds of practitioners and by reconciling their objective and subjective perceptions" (p.177). The embedded nature of human existence where cognition is considered an outcome of social interrelationships, assumes a constitutive ontology where understanding is a result of active reflection and decision making is the result of the legitimate nature of an experience (Maturana 1988). Learning, through knowing, may therefore be seen to involve individual reflection on experience, sensory perceptions and active doing, with communicative dialogue in a world of others, facilitating a collective conscious.

However, it is proposed, that what limits the effectiveness of pragmatic knowledge and adds to the complexity of an already ambiguous praxis of living is the reality the there are actors that function within a 'transcendental' ontology (Maturana 1988), defining their reality and validating their experience against an overarching metanarrative or belief system. If individuals look to an entity to define their existence (e.g. religion, nature, tradition), this perpetuates an ontological dualism where, as a human entity, they remain independent from what defines knowledgeable truth. Aligning this 
with pragmatic knowledge where cognition is enacted and emergent, it is suggested that unless individuals function in a domain of 'constitutive' ontology (Maturana 1988) where a state of 'becoming' is facilitated through ongoing reflection, a static state of 'being' in a world remains.

Relating this to a project environment, where projects are socially constituted, the challenge for project managers working with socially-complex project teams will be to address the ontological differences that arise from human plurality. It is managing the discursive dialogue, which reflects differences in interests, goals and beliefs, which is the challenge. If individuals can be identified as belonging to the domain of constitutive ontology where knowledge is not considered finite and there are no intrinsic falsehoods, then facilitating interconnections of human realities for emergent change seems feasible. However, if individuals working together in a project environment exist in quite contrasting praxis domains, with some believing that realities which cannot be explained by a universal entity are intrinsically false, whilst other individuals are open to adapting beliefs through sharing realities and altering justifications accordingly, then the situational reality may be irreconcilable. Whilst it has been said that “... knowledge doesn't so much reflect a state of the world but acts to shape it in ways that both facilitate and constrain action" (Healy 2003 p. 690), there needs to be recognition that there are real barriers to emergent change, when the very basis on which individuals base their praxis of living differ greatly.

\subsection{The changing nature of organizations}

The concept of sense-making for organizational transformation in a project management setting necessitates consideration of the nature of 'organizations', the role projects play in organizational functionality and the positioning of the organization in relation to its environment. This section is divided into four subsections, with discussions being informed by the conceptual framework defined in section 2.2. The first subsection gives consideration to the cognitive nature of the knowledge based organization, the second then reviews the organization as project oriented, whilst the third considers the relevance of learning for an organization and its associated capacity. The fourth subsection then introduces the concept of quality and effectiveness in relation to organizational resilience in the face of complexity and change.

\subsubsection{The cognitive nature of the knowledge based organization}

The conceptual framework for this thesis (Figure 4) supports a shift in thinking about organizations as rational socially constructed entities comprising linear processes and static functionality (Cooper and Burrell 1988), to a view which appreciates the complexity, ambiguity, uncertainty of the world in which we live. The framework also encourages thinking of organizations as language, discourse, 
chaotic narratives (Hazen 2001); as instruments and products of change (Hasselbladh and Kallinikos 2000; Dambrin, Lambert et al. 2007); and as disorganized open human activity systems where cognition, the knowing how, assists in interpretation and decision-making (Höijer, Lidskog et al. 2006; Ivory, Alderman et al. 2006). An enactivist paradigm complements postmodern thinking through portraying cognition as inseparable from an embodied system, where "Embodiment is a condition sine qua non for all knowledge, including the complex patterns of cultural knowledge" (Baerveldt and Verheggen 1999 p.189) with reality emerging through a domain of constitutive ontology (Maturana 1988).

It is surmised that organizations as socially constructed entities may be seen to exist as autopoietic, organizationally closed yet structurally open through being coupled within an environment, where knowledge creation results from interrelationships of multiple realities actively seeking meaning through circular sensemaking behaviour (Weick 2001). The autonomous nature of the organization assumes it exists in a phenomenal domain, functioning as a cognitive system whereby knowledge is enacted to produce meaning rather than process information (Baerveldt and Verheggen 1999). Cognition as knowing is derived from languaging, assuming "...nothing exists in human life outside language because human life takes place in language" (Maturana 1995 p.2), with meaning being relational and circular and knowledge manifesting itself in 'effective action' (von Glasersfeld 1979). Thus sense making may be seen as a linguistic process (Weick 2001; Phillips, Lawrence et al. 2004), with interpretations for emerging adaptations taking place through individual, group and organizational multilevel learning as a result of action (Crossan, Lane et al. 1999).

Organizations have traditionally been considered as 'complex social systems' (Weick 2001; Mullins 2005), however, there has been a greater emphasis on the structural and functional elements of the entity, rather than on the interconnected and embedded nature of humans and cognitive processes within a socio-political environment. In an enactivist paradigm an autonomous system is one which is capable of maintaining its own organization whilst being structurally flexible to facilitate adaptation to environmental contexts. This structural openness involves a cognitive domain of interactions in which the system exists without losing its identity (Baerveldt and Verheggen 1997), yet the embedded relationship of 'organization in environment' suggests the presence of a vulnerability for an institution due to the influence and impact fluctuations in social and cultural factors may have on business practice.

The cybernetic concept of the organization proposed by Varela (1979), identifies autonomy and control as the two major forms of knowledge which may be reflected in organizational discourse. Models of sensemaking that overlook the intersubjective realities of knowledge flow taking place at all levels of the organization, are limiting, as they seemingly perceive information as controlled and referential. This "... restricts the point of view to fixed interactions and observational positions, it is 
'instructive'..." (Cooper and Burrell 1988 p.93) and tends to ignore the role of the observer in knowledge construction through telling the players how to act. Discourse on knowledge and information which accommodates complexity and plurality, assumes an autonomous approach, where information is considered self-referential and "... always relative to the process of interactions of the domain in which they occur, and to the observer-community that describes them..." (Varela 1979 p. 267). Postmodern discourse focuses on interpretations of the world without attempts at explaining these through an overarching metanarrative. The human element in organizations cannot be ignored, nor can thinking be controlled and objectively managed. The wealth of an organization may be found in the multiple and often competing realities of actors in an organization and it is through acknowledging the value inherent in individual interpretations of the world, that autonomy in organizational responsiveness may be facilitated.

Weber's (1978) model of organizations as bureaucracies portray the modern organization as a process which reflects objective thinking dominating positivist views of social life (Cooper and Burrell 1988). In such models, the rationality and logic embedded in functional aspects of organizational systems limit the capacity of an organization to cater for emerging changes or spontaneity. Active learning in the emerging organization will "...reveal the precarious local orchestration of material, technical and social relationships which give rise to relatively stabilized configurations..." (Chia 1995 p.601), with such knowledge based organizations being able to move beyond just accommodating for difference and diversity, to actually utilizing 'competing narratives' (Boddy and Paton 2004) for creativity, innovation and transformation.

Postmodern thinking coupled with an enactivist paradigm moves organizational perception from grand narratives and metadiscourses which attempt to explain organizational functionality in logical terms, to a thinking which advocates for "...the existence of a license to autonomous, idiosyncratic, creative and transgressing behavior in any organization" (Kreiner 1992 p.37). This subsequently provides an incredulity to dispute the legitimacy of reification of entities and notions of knowledge in preference for a plurality of small narratives that compete with each other to facilitate learning (Lyotard 1979). The rapid changes in the way we 'do' business in part created by advances in technological communication systems, ease of global travel and rise in multinational corporations, create inconsistencies, ambiguities and dilemmas in decision making. The elements of predictability and certainty which were once reflected in classical Weberian organizational theory are now missing in an era exhibiting a plurality in discourse and complexity in knowledge. Certainty has been replaced with uncertainty, stability with instability, simplicity with complexity. "Uncertainty and surprise is part of the game and you need to be prepared for it and learn to live with it" (Folke 2006 p. 255). 
It is proposed that recursive learning underpins emerging realities which in turn reveal organizations as emerging entities in an uncertain world. Although interpretations and ascription of meaning to phenomena remain idiosyncratic and in the realm of the individual (Rosenau 1991), assimilation of meaning may be facilitated through visualizing organizational knowledge flow as interconnecting spirals of interpreted information, where consensus actions may be possible despite discursive sensemaking. The organization of the future, as a reflection of the society of the future, will fall "... less within the province of a Newtonian anthropology (such as structuralism or systems theory) than a pragmatics of language particles" (Lyotard 2004 p.2). Communication assumes knowledge flow through language, with knowledge as a by-product of individual interpretation being subjectively communicated and in turn subjectively interpreted by another. Postmodern inquiry does not assume there is an ultimate truth being conveyed through communication, rather suggests that language transforms truth into a "largely linguistic convention" (Rosenau 1991) where language is primarily situated and "... can only be known to the person speaking; to his immediate private sensations ... " (Wittgenstein 1953 Part 1 p. 243). Modernists would apply some sort of metanarrative framework in the attempt to give 'shared' and objective meaning to language discourse. However, if we acknowledge the embodied nature of cognition in socio-culturally complex contexts, pure consensual meaning will always be elusive due to the unique and subjective character of human interpretation. Human conduct where the actor's psyche is embodied and inextricably linked with other actors in a social world, may be culturally patterned, yet remain individually authentic due to the uniqueness and subjective nature of human experience (Baerveldt and Verheggen 1997). It is the management of such socio-cultural complexity which creates challenges in decision making, particularly given the inevitable overt and covert power relationships which exist in socially messy project organizations grappling with constituting useful knowledge despite human plurality (Marshall 2006).

Postmodern discourse assumes no foundation for reason or rationality to underpin construction of new knowledge within an organization, as this would necessitate some sort of linguistic metanarrative. Rather, knowledge remains in the realm of uncertainty, complexity, ambiguity, selfreferral and autonomy, which creates challenges for organizations in determining their capacity to act. There appears limited literature in relation to what social structures or techniques can facilitate knowledge sharing for sensemaking in culturally complex working and project environments (Bresnen, Edelman et al. 2003). This research proposes that assumptions underpinning an enactivist paradigm particularly in relation to autonomy of living systems determining the domain of cognition (Baerveldt and Verheggen 1997), supports the embedded nature of action and understanding to facilitate sensemaking for potentially consensual worlds despite cultural differences. Clark and Geppert (2006) make reference to knowledge transfer and learning processes as underpinning organizational transformation in culturally asymmetrical contexts, comparing company relationships involving polycentric and ethnocentric partner orientations. The diversity and complexity in context 
created by divergent cultural groups has also been identified by Clark and Geppert (2006) as contributing to power relations and the precarious nature of organizational change. Whilst their research primarily focused on knowledge transfer between local post-socialist communities and western multinational corporations involved in long term contract work, there are a number of factors in relation to the impact of contextual asymmetries which are applicable to knowledge and learning processes in other 'emerging market economies', and which are relevant to this discussion.

The valuing of knowledge as an element integral to organizational effectiveness has increased in awareness parallel to a reduction in institutions depending on manual labour due to industrial mechanization. A shift in emphasis has taken place over the last few decades from the manual worker whose effectiveness is measured by accuracy of output based on instructions, to that of a 'knowledge worker' "...who puts to work what he has between his ears rather than the brawn of his muscles or the skill of his hands" (Drucker 1967 p.3). He is a worker that produces ideas and information rather than a physical product, which is quite different to the thinking underlying scientific management theory early $20^{\text {th }}$ century (Taylor 1911 ).

Despite a perpetuation of positivist approaches being applied to organizational analysis through rational models of functionality, there have been sizeable shifts in ontological thinking in relation to the nature of human existence in the actuality of organisations. A more 'humanistic' approach to organizational theory was originally introduced ten to twenty years after Taylor's theory, as a result of the Hawthorn experiments of George Elton Mayo (1933) which focused on social behavior and human motivational factors on productivity within the company. Despite criticisms of his experimental design, Mayo's work has been noted as initiating a shift away from the objective nature of scientific experimentation (Clegg and Dunkerley 1980; Crowther and Green 2004). In time however, as noted by Nonaka and Takeuchi (1995), even Mayo's theories of human relations were later objectified into more behaviourist models of learning, reinforcing the Cartesian mind-body split of the $17^{\text {th }}$ century Descartian tradition of dualism of rational knowledge (Descartes 1637). Whilst other theorists attempted to 'heal' this split and introduce a more humanistic approach into organizational functioning (Barnard 1938; Simon 1945; Cohen, March et al. 1972; Weick 2001), the "...view of knowledge in the science of strategy is similar to that of Taylorism" (Nonaka and Takeuchi 1995 p.41) and fails to adequately connect the human and cultural elements in organizational functioning to knowledge creation and flow.

The concept of the knowledge worker and knowledge society (Drucker 1967) remain pivotal to considering today's knowledge based organization as an active, transformational socially constructed entity, which has the potential to evolve and change according to individual workers sensing and interpreting the world around them. The emergent nature of cognition facilitates conceptualizing knowledge as a cognitive process, rather than being an entity which can be codified, stored and 
transferred, with the work of Varela (2000) as summarized by Limone and Bastias (2006 p.43), clarifying what cognition actually is:

- Effective action in a domain with a history of structural coupling that generates a world;

- Functioning through a network of interconnected elements capable of structural changes that experience an uninterrupted history;

- Functioning properly when it becomes part of a world of continuous and existing meaning (in ontogeny) or forms a new meaning (in phylogeny).

This cognitive process as applied to the knowledge-based organization, therefore assumes emerging knowledge, thus an emergent organization, which is continually creating, re-creating and transforming in response to contextual factors sensed in the environment. Individuals may be seen as structurally coupled with each other and the environment, exhibiting actively seeking behaviour for knowledge creation. Knowledge thus emerges as key to organizational survival given the more complex and diverse working environments of today, where "...inconsistencies, contradictory demands, and dilemmas in decision-making" (Eijnatten 2004 p. 430) dominate.

A globalized market economy, together with technological developments in communication systems and rapid pace of business, have created new challenges for organizations as they are pressured to strategically manage corporate assets to ensure competitive advantage is maintained and future sustainability is assured. It has been suggested that wise management of knowledge as an intangible resource and key asset is the lynch-pin to corporate success and survival (Nonaka and Takeuchi 1995; Teece 1998; Hansen, Nohria et al. 1999; Zack 1999; Davenport and Prusak 2000; Scheepers, Venkitachalam et al. 2004; Lin and Tseng 2005; Macpherson 2005; Jashapara 2007). Yet attempting to control this 'knowledge' given the autonomous nature of organizations facing increasingly ambiguous working environments is challenging. Strategic approaches to ways of doing business that demonstrate an appreciation of the embedded nature of individual and collective knowledge resulting from interpretation and sensemaking, are necessary for optimizing an organization's capacity to act. It has been suggested that knowledge as complex capital resides in the human element of the organization so that attempts to measure and monitor flow, given its embedded, tacit and subjective nature, add to the uncertainties of the evolving business environment today (Nonaka and Takeuchi 1995; Argyris 1999; Davenport and Prusak 2000; Hall and Paradice 2005). The resilience of an organization may therefore be seen to be dependent upon not only the recognition of knowledge as a key resource, but through ensuring relevant governance systems are developed and adequately adaptive (Folke 2006).

Exploration into 'optimizing' and 'strategically managing' knowledge as an asset has received equal attention in the literature (Zack 1999; Cavaleri and Reed 2000; Cavaleri 2004; Walker 2004; Hughes 2005; Walker and Christenson 2005; Choi, Poon et al. 2008). Whilst to a relatively lesser extent, 
research on associating knowledge flow with organizational learning and effectiveness is evident (Bass and Avolio 1994; Nahapiet 1998; Argyris 1999; Kakabadse, Kouzmin et al. 2001; Iskander 2004; Cavaleri, Seivert et al. 2005). The link between knowledge and performance is more elusive due to difficulties measuring the effectiveness and organizational capability given the intangible and embodied nature of knowledge. Empirical research still tends to favour rational exploration of reifying knowledge within a 'hard thinking' paradigm focusing on control and rationality. Whereas this dissertation proposes that through using an exploratory 'soft paradigm' approach to investigate problem situations in organizational analysis, that a shift in valuing knowledge in relation to learning, change and emerging entities may be facilitated (Checkland and Poulter 2006; Pollack 2007; van Donk and Molloy 2008).

The current era of 'globalisation' which affects knowledge flow on an international scale, underpins the social changes evident in 'being' in a world which is ontologically pluralistic. Most commonly, globalisation is associated with processes that connect individuals in an international arena where there is an "...intensification of worldwide social relations which link distant localities in such a way that local happenings are shaped by events occurring many miles away and vice versa" (Held 1991 p.9). The dominant recurring themes are political shifts in power, expansive economic practice and the cultural impact of more complex and diverse epistemology (Ladwig 1994; Morrow and Torres 2000; Healy 2003; Lingard, Rawolle et al. 2005; Chuah, Hoffmann et al. 2007). National borders are no longer impervious to the effects of global trade, with developments in communication technology facilitating the pace and breadth of information and knowledge flow. 'Globalization' is indicative of a 'world-wide interconnectedness' in all aspects of life (Held, McGrew et al. 1999) where transience in all forms of capital - human, economic, technological, organizational - has created an imperative for organisations to move beyond systems of control for sustainability, to social-ecological systems which facilitate change for resilience (Folke 2006).

In this context, knowledge is seen as the 'new' resource despite it having always been present in the human element of organizational life. It is argued that it is through the valuing and recognition of knowledge as an active and changing resource that an organization's capacity to act is ensured as it emerges and evolves in an era of chaos and unpredictability. "In all developed countries, knowledge workers have already become the centre of gravity of the labour force..." (Drucker 1986 p.35), and it is these workers who actively move beyond working with data and information, to create the future. This is the knowledge based organization. An autonomous organization with boundaries made permeable through project practice, where all workers are encouraged and supported to actively inquire and make sense of the world around them. Knowledge as an advantage and asset is not finite and static. It grows and emerges and is embodied in language, reflection and dialogue, with learning and unlearning as recursive interconnected processes underpinning any sustainable 
advantage for the knowledge organization (Cavaleri and Fearon 1996; Davenport and Prusak 2000). As suggested, globalization brings a social complexity to nation states as a result of different ways of knowing and doing being absorbed and assimilated, with a subsequent reduction in importance of a nation-state's own dominant culture to organizational functioning (Crowther and Green 2004). Organizations are now the 'becoming' cognitive systems whose resilience is dependent upon producing meaning as opposed to processing information (Baerveldt and Verheggen 1999), with value being found in project practice where knowledge as action replaces knowledge as objectively finite.

\subsubsection{The project organisation}

Throughout the $20^{\text {th }}$ century, work environments have increased in complexity, technology has altered the pace of business and the effects of globalization have created a more diverse marketplace resulting from changes in socio-economics. This in turn has seemingly affected organizational structure and workplace behavior (DiTomaso and Hooijberg 1996; Bass and Steidlmeier 1999; Osborn, Hunt et al. 2002; Avolio, Gardner et al. 2004), with traditional bureaucratic organizational structures being noted as impeding rapidity of response to current business demands. Consequently there has been evidence of a shift to more flexible flatter organizational structures with functional emphases on project based management (Turner 1999; Hodgson and Cicmil 2006). Both large and small organizations are experiencing pressures to work faster and smarter and in this context we see the increasing profile of the project manager; “... management by projects has become the new general management. The bureaucratic organization, introduced in the nineteenth century to provide efficiency through permanence can no longer respond to the competitive environment of modern business" (Turner 1999 p. xix)

In this era of complexity and constant change, business by projects or through projects have been noted as being better able to accommodate the rapidity of change as well as function in a temporary capacity to actually facilitate change (Turner 1999; Hodgson and Cicmil 2006; Hobbs, Aubry et al. 2008). Over the last ten years the nature of projects in particular has come under investigation, with there being evidence of thinking of projects as encompassing part of a systems approach to organizational analysis. Projects have been defined, as 'tools' for business (Packendorff 1995; Winter, Smith et al. 2006); as temporary organizations (Thomas, Clark et al. 1993; Lundin and Söderholm 1995; Turner and Müller 2003; Modig 2007); as emerging, initiators of organizational learning and change (Kreiner 1992; Bresnen 2006); as objects of organizational innovation (Hobbs, Aubry et al. 2008); and as language and practice (Lineham and Kavanagh 2006). Postmodern thinking also facilitates movement from viewing projects as endeavours which are goal directed, structured, 
planned within a time frame and unique (Turner 1999) to considering them in an ontology of becoming (Chia 1995) as "... an emergent outcome of disparate, ambiguous, political practices" (Lineham and Kavanagh 2006 p.55). This does not completely negate the defining characteristics in traditional systems theories of project management, but rather provides a different and perhaps more relevant framework to consider projects in relation to action, movement, knowledge flow and change.

Whilst the concept of a project as a 'temporary organization' which "... is incessantly enacted by individuals continuously learning by experience and expecting further learning" (Packendorff 1995 p.328) has facilitated movement from more static models of project practice, project management models traditionally still involve set stages of 'development, implementation and termination' (Söderlund 2004a). The temporary nature of the project has drawn on organizational theory to explain functionality, primarily due to the absence of a strong project management theoretical base (Kreiner 1992; Lundin and Söderholm 1995; Turner and Müller 2003; Söderlund 2004a; Modig 2007). Projects as 'temporary organisations' are presented as non-static temporary entities which are purposefully enacted to solve complex problems within a 'stationary' or stable permanent organization (Modig 2007). Lundin and Söderholm (1995) identify four specific characteristics of projects which distinguish them from the permanent organization - time, task, team and transition. They note that "Permanent organizations are more naturally defined by goals (rather than tasks), survival (rather than time), working organization (rather than team) and production processes and continual development (rather than transition" (p. 439). Although aligning projects with organizational theory may assist in conceptualizing the functionality of projects as 'vehicles' or 'vectors' of strategy and competency development (Bredillet 2008), epistemologically there is still a tendency to rationalize projects as temporary organizations which are in a state of 'existence' or being, rather than as enacted interconnected entities which facilitate organizational emergence and a fluid state of 'becoming'.

Turner and Müller (2003) have also noted that presenting projects simply as 'temporary organizations' which are unique, transient and utilize novel processes, does not fully explain the functionality and limitations of projects. They emphasize the active component of projects as agencies for change, resource utilization and uncertainty management, which may be seen to highlight the issue of the multi-level relationships between individual employees, the project, the organization and the environment. The human element in projects (as opposed to the technical) is considered critical given the role of knowledge flow and continuous learning which takes place, and this is what appears to be lacking in project conceptual theory to date.

Although Turner (1999) has identified project management as becoming more mainstream in organizational management, there seems to be a tendency to focus more on the processes of 
achieving set goals, rather than attending to complexities that exist particularly in the field of human relationships. Acknowledging the interconnected nature of cognition and language as being inherent to learning and change, there is a need to more comprehensively explore the functionality of the project unit and organization (Söderlund 2004a) to ascertain barriers and conduits to knowledge flow amidst complexity and ambiguities. Turner and Müller (2003) have suggested that projects as temporary organizations assist business practice through resources being assigned "...to undertake a unique, novel and transient endeavour managing the inherent uncertainty and need for integration in order to deliver beneficial objectives of change" (p.7). It is the management of this 'uncertainty' and 'need for integration' caused by a multiplicity in meanings and discursive dialogue which warrants further exploration.

Winter et al (2006) have indicated that "... the conceptual base of project management continues to attract criticism for its lack of relevance to practice..." (p.638), with there needing to be a greater emphasis on the social realities existing within specific project contexts. As a result of Winter et al's (2006) funded research, they identified five research directions which have the potential to expand the theoretical base for project management practice.

The directions encompass three areas - theory about practice, theory for practice and theory in practice. With the different theoretical bases for each of these areas being influenced by whether knowledge of the project as process is 'pre-existing' or 'given' or whether the project research process emerges as a 'becoming' entity. The future research model suggests an evolving position 'from' what exists in current research 'towards' an enhanced position. These concepts reflect post modern thinking where an ontology of movement, action and emerging processes (Chia 1995) dominate, thus facilitating an appreciation of projects as emerging activity systems working in and through complex non-static evolving environments. Winter et al's (2006) principle findings indicate that there is "... the need for new thinking in the areas of project complexity, social process, value creation project conceptualisation and practitioner development" (p. 641) and it is within this framework of thought that the following research thesis resides. The aim is to emphasise projects as social process, moving away from instrumentality of projects as linear and predictable, to highlight the complex nature of human interrelations, the movement of human actions and the real and hidden social agendas which influence project process and success. It is surmised that socio-cultural factors in project contexts impinge upon reflective learning practices which are integral to adaptive organizational change, with organizations as 'complex adaptive systems' being supported by project practice absorbing environmental perturbations and facilitating regeneration, reorganization and renewal for organizational transformation and resilience (Folke 2006).

An ontology of 'becoming' is able to support the view of learning as enacted 'in and through' projects as emerging entities, where projects may be seen as 'language' \& secondly as 'practice', with the 
active element of languaging facilitating knowledge construction and flow within organizational communities (Lineham and Kavanagh 2006). "The shifting view of language from representation to construction, from a container of knowledge to a constructor of realities, moves us from asking 'what do we know about the world?' to 'what language games do we participate in and what are the consequence and prizes attached to such games?"' (Lineham and Kavanagh 2006 p.59). The challenge for those working in culturally pluralistic project environments is to make sense of multiple realities in a multiplicity of language, to try to determine a common ground for understanding, to find rationality amongst irrationality and to facilitate the flow of knowledge for meaningful interactions.

Re-directing project theory encourages a re-think on the relationship between the individual and the organization (Kreiner 1992), raising such issues as organizational autonomy and control, selfregulation and change. "Projects act as vehicles to develop competencies (individual, team, and organizational) when modifying its environment and thus lead to competitive advantage and value creation" (Bredillet 2008 p.239). Projects can thus be used strategically to not only 'solve' problems in the organizational environment, but to facilitate change through inquiry and reflection to create knowledge for optimizing the capacity to act as a social process. The embedded nature of action in projects supports a pragmatic approach to sensemaking (Weick 2001) where enaction is pivotal to intersubjective realities connecting and acting to make things happen to promote adaptation and renewal for organizational sustainability and resilience (Folke 2006).

Projects may therefore be seen as a conduit for organizational transformation, through facilitating structural coupling within an environmental context. As represented in Figure 6 whilst the organization and project entity per se remains 'organisationally closed', the project structure is open, mutually coupled both with the organization and the environment, actively functioning in forward directed sensemaking activities whilst feeding back interpreted information to the main organization. 


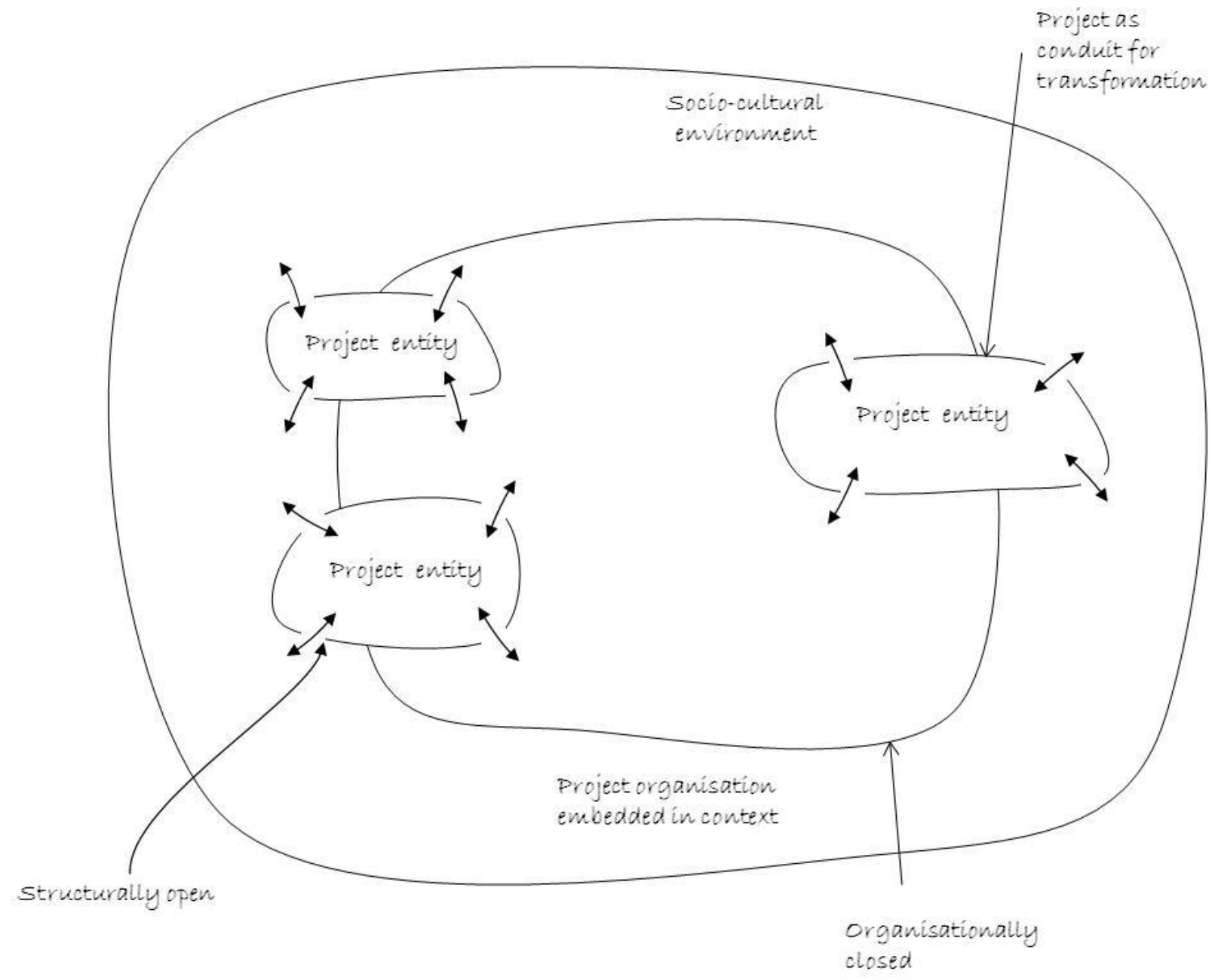

Figure 6 The Open Project Structure

Such interplay promotes a balanced and circular nature to project existence, akin to metabolic changes in living organisms, to ensure organizational equilibrium is maintained whilst incremental adaptations to contextual change is made possible. Visualising the project organization as autopoietic in nature, even if only metaphorically, can thus support the conceptualization of project existence as potentially ontologically autonomous, yet as an entity whose survival or resilience is dependent upon a self-perpetuating relationship with and within an organizational entity. The embodied nature of cognition in projects, then provides the 'stickiness' to hold relational strands together, as circular knowledge flows facilitate movement from a state of ontological resilient being to one of transformational becoming as represented in Figure 7. 


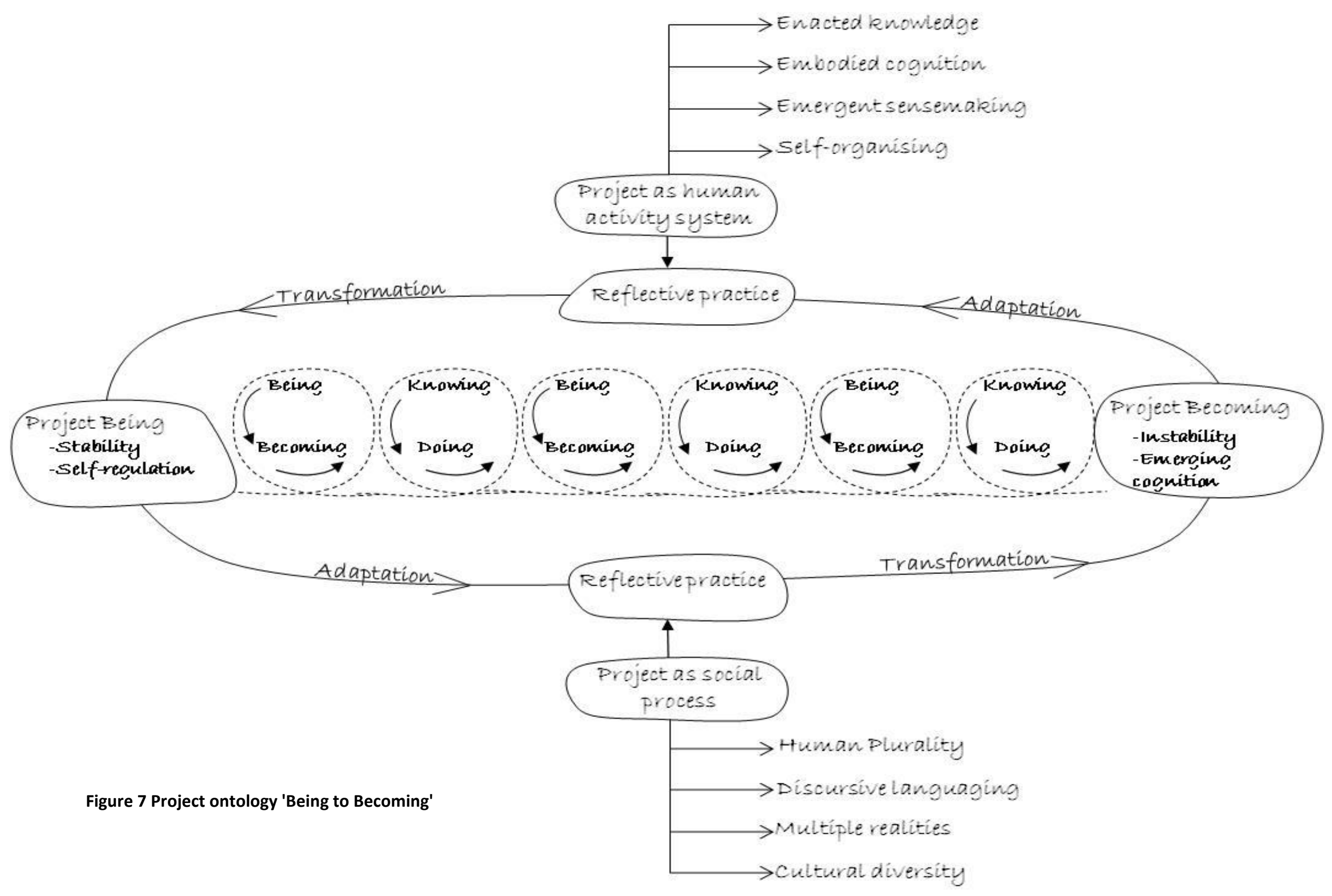




\subsubsection{Organizational capacity to learn}

Despite some disparity in the field of 'organizational learning' research (Cavaleri and Fearon 1996; Moingeon 1996; Argyris 1999; Crossan, Lane et al. 1999; Wenger 1999; Cohen 2001; van Eijnatten 2004), a consistency in definition has been identified by Boreham and Morgan (2004). “...learning as organizational ... is undertaken by members of an organization to achieve organizational purposes, takes place in teams or other small groups, is distributed widely throughout the organization and embeds its outcomes in the organization's system, structures and culture" (p. 308). The important elements here include the 'membership' of the organization, the emphasis on team work or collaborative small group experience and the assimilation of new knowledge into the ways of business practice. With knowledge being identified as the 'new' resource of the firm (Daft 1983; Wernerfelt 1984; Barney 1991), a plethora of research on defining what knowledge actually is, how it can be created, exploited, codified, transferred, all for the benefit of the company has dominated organizational analyses over the past couple of decades (Winter 1987; Prahalad and Hamel 1990; Nonaka and Takeuchi 1995; Spender 1996; Teece 1998; Cohen 2001).

The phrase 'learning organization' was introduced by Senge (1990) to identify an organization "...where people continually expand their capacity to create the results they truly desire, where new and expansive patterns of thinking are nurtured, where collective aspiration is set free and where people are continually learning how to learn together" (p. 4). This learning assumes the presence and utilization of multi-level knowledge for individual, group and organizational growth (Nonaka and Takeuchi 1995; Crossan, Lane et al. 1999), with knowledge flow within and between these interconnecting intraorganizational elements existing within an external socio-political environment. The quality of the interconnections and the strategic management of the creation, flow and storage of knowledge, supposedly reflect an organization's capabilities in responding to changing business demands. That is, organizational learning is seen as "... the capability of an organization to process knowledge... to create, acquire, transfer, and integrate knowledge, and to modify its behavior to reflect the new cognitive situation, with a view to improving its performance" (Jerez-Gomez, Cespedes-Lorente et al. 2005 p.716). However, what seems to be missing from these definitions is recognition of the embedded nature of organizational cognition as pivotal in accommodating for socio-contextual complexities. Emerging realities and organizational resilience necessitate systems of management and governance which have the capacity to renew, create and be innovative in an era of uncertainty and dynamic change (Folke 2006).

Most organizational learning theory models follow a systems theory approach (von Bertalanaffy 1968), where the 'organisation' as a learning entity is objectified as a recognizable system and 
knowledge is presented as definable and controllable. Predominant learning models are of a cyclic or spiral nature, reinforcing learning processes that involve observation or scanning, interpretation or assessment, action or implementation, followed by evaluation or repeated observation (Kim 1993; Nonaka and Takeuchi 1995; Argyris and Schön 1996; Crossan, Lane et al. 1999; Daft and Weick 2001). In turn, elements of these circular learning frameworks have been combined to create and represent more detailed processes of organizational learning (Hall 2005; Järvinen and Poikela 2005) yet the complexities of context resulting from mixes of human socio-cultural plurality, remain challenging to incorporate. "Factors such as beliefs, politics, goals and perceptions may complicate the organisational learning cycle..." (Weick 2001 p. 245), with it being suggested that the variances in learning styles and cultural typologies which positively correlate (Yamazaki 2005) need to be accommodated for when managing project organizations as emerging social-ecological systems (Folke 2006).

Through application of an enactivist theory of cognition, it is possible to move closer to reconceptualizing organizations as being complex adaptive learning systems embedded in context. Parboteeah and Jackson (2007) have created an autopoietic organizational learning model expanding upon Kim's (1993) Observe, Assess, Design, Implement (OADI) learning cycle, which in turn drew upon existing theory incorporating the Lewinian experiential model (Kolb 1984), single-loop and double-loop learning (Argyris and Schön 1996), Johnson-Laird's (1983) theory of mental models and March and Olsen's (1975) 'Organisational Learning under Ambiguity' model. Parboteeah and Jackson's (2007) model applies the concept of autopoietic systems to individual and organizational learning, particularly noting the circularity in process which is characteristic of such autonomous systems (Figure 8).

Varela, Maturana et al (1974) identified six characteristics that complex systems must exhibit to be considered autopoietic or autonomous systems, as paraphrased by Hall (2005 p.170) as directly quoted:

1) The system is discriminable from its environment. Components belonging to the system can be discriminated from environmental components by the system and external observers.

2) The components of the system are determined by the system. Components belonging to the system are identified as such by processes intrinsic to the system (self-reference).

3) The system is dynamic. Interactions and transformation of components belonging to the system are determined by the system.

4) The system dynamically maintains its identity. System processes work to maintain the integrity of the system. 
5) The system intrinsically produces its own components. Components from the internal or external environment are altered or transformed by system processes to make them functionally and identifiably parts of the system.

6) The components produced by and forming the system are necessary and sufficient to produce the system. The system's self-production is autonomous.

Giving the nature of organizations as socially constructed entities comprising humans involved in social interactive relationships, it is felt that presenting organizations as autopoietic 'living systems' as defined by these listed characteristics (Hall 2005; Limone and Bastias 2006) supports a socialecological view of institutions and people (Folke 2006) to underpin a framework which notes the “...dynamic complexity of organizational change" (Hobbs, Aubry et al. 2008 p.550).

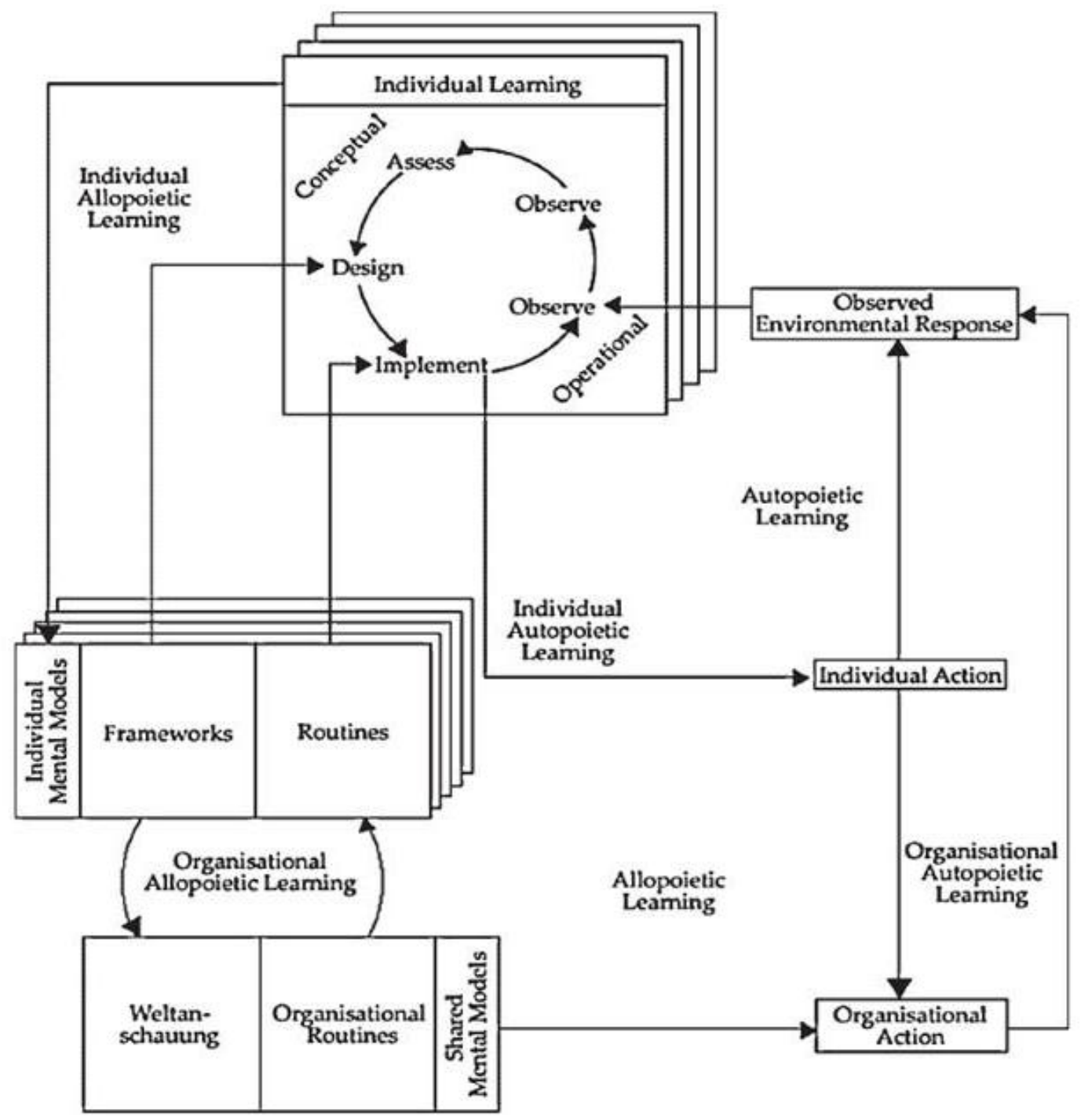

Figure 8 Autopoietic organisational learning model (sourced Parboteeah \& Jackson, 2007 p257) 
Although the nature of social organizations as autopoietic has been disputed (Hejl 1980), with Varela (1979) and Maturana (2002) challenging the transference of their biological theory of self-producing and re-generating living systems to institutional organizational systems, other authors have preferred to highlight the theoretical similarities in relation to the nature of cognition, self-perpetuation and system autonomy (Baerveldt and Verheggen 1997; Baerveldt and Verheggen 1999). Rather than becoming entangled in debate as to whether organizations function autonomously without input or output, there are others who find the metaphorical perspective valuable even if all elements of autopoietic theory are not transferable to organizational analyses (Kay 2001). Enactivist theory supports the concept of organizations as operationally closed autonomous systems operating within a phenomenal domain distinct from its background (Baerveldt and Verheggen 1999); with autopoiesis representing the organizational circularity of living systems as a unit of interactions where order is maintained through a domain of interactions. Organisations as social systems as 'living cognitive systems' where "...living as a process is a process of cognition" (Maturana 1980 p.13), serves to provide a useful model for organizational transformation through presenting cognition as an emergent process.

It is in this context that Parboteeah and Jackson (2007) designed their Autopoietic organizational learning model, distinguishing between allopoietic and autopoietic individual learning and processes, suggesting that people are first order autopoietic entities and organizations second order. This model is useful in conceptualizing the complexity of learning processes, particularly in the context of organizational learning. However, although reference to an observed environmental response is aligned with shared mental models and allopoietic learning is alluded to, with individual learning linked with action, it is not clear in their paper as to how the actual 'sharing' of mental models may be facilitated, nor how learning through reflective practice would function in socially complex working environments. Furthermore, given the rapidity of change in today's increasingly changing business world, although the model is aimed at assisting companies understand the workings of their organization, it is not obvious how emergent change for organizational resilience as opposed to survival through this learning model, would be achieved.

It is proposed that 'learning' both individually and in turn organizationally, may be envisaged more simply as a process of transformation facilitated by 'gestures of becoming aware' (Scharmer and Varela 2000), enacted at different levels of the organization, individual, group and system. Learning as emergent and transformational is based more on interconnecting cyclic learning processes as opposed to 'stand alone' closed models, with learning being more accurately portrayed as inseparable from context where "... each individual can actually access his or her experience" (Scharmer and Varela 2000 p. 4). Such a model of awareness developed by Depraz, Varela and Vermersch (1999), incorporates a triangulated approach to explore experience as an individual 
subjective process which cannot be 'observed' due to emergent actions only being definable by 'being in them'. Elements of suspension, redirection and letting-go (Figure 9) interconnect as informed by methodologies of introspection, phenomenology and contemplative traditions, to create an epochè where existence and action in the world is momentarily suspended to facilitate a blank slate in which to consider the world. This model, which Varela has noted as being an awareness process, is embedded in individual experience. However, it necessitates social mediation, is essential for language and social learning, and is mediated by context (Scharmer and Varela 2000).

\section{epochè}

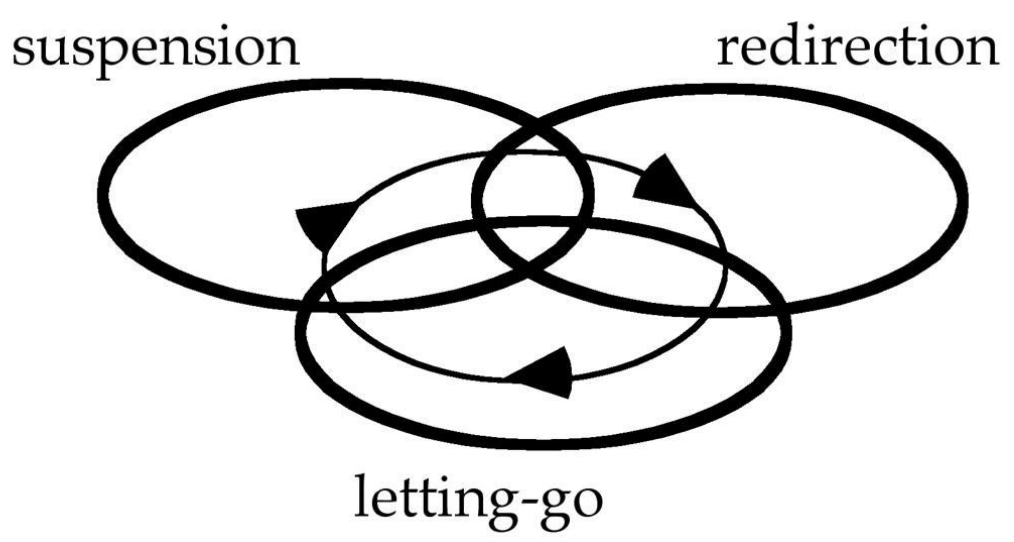

Figure 9 The core process of becoming aware (sourced Scharmer \& Varela 2000 p.4)

The 'three gestures of becoming aware' assume an embodied view of cognition, with learning being individual yet intertwined with multiple realities, moving beyond an observer definition of knowledge being 'out there'. Individuals may be perceived as structurally coupled with one another and with an environment thus more accurately portraying the interconnectedness of the human element in social environments. Cognition then acts as the modus operandi of organizational survival or resilience (Holling 1973) to support "...homeostatic processes within the system responding to perturbations in order to maintain the entity's capacity for self-maintenance and self-sustainment" (Hall 2005 p.171). This 'awareness' model seems a better fit when considering projects as human activity systems, whereby projects as praxis could be seen to facilitate the social mediation necessary for learning as emergent cognition in ambiguous, socially messy working environments.

Given the complex and dynamic nature of social processes and the realities of human knowledge as embedded organizational capital, continuing to view learning in linear, cyclic or unidirectional terms 
seemingly limits the comprehension of an organization's transformational capacity. The emergent nature of individual and collective consciousness needs to be acknowledged, with cognition as the definable characteristic of living systems. Resilience of an organization will then directly correlate with a system's ability to self-organize noting the inseparable nature of cognition from context. "...all knowledge may be emergent from, embedded in, distributed across, and inseparable from real time processes of perceiving, remembering, attending and acting" (Smith 2005 p.279). The foundations of project management lie in large scale engineering, construction and IT projects (Turner 1999), where resilience may be seen to focus on efficiency, system constancy and predictability to resist change and maintain a balanced state for sustainability (Folke 2006). This linear approach is considered inadequate given the realities of projects as socially complex systems, where disturbance and sensing change in the environment necessitates adaptive responses that involve a different form of resilience based on capacity for flexibility, change and renewal.

The organizational capacity for change through learning has also been associated with an organization's capability “... to process knowledge ... to create, acquire, transfer and integrate knowledge and to modify its behavior to reflect the new cognitive situation, with a view to improving its performance" (Jerez-Gomez, Cespedes-Lorente et al. 2005 p. 716). Although 'knowledge' in such a definition remains objectified, the link between knowledge and change continues to be useful. JerezGomez, Cespedes-Lorente et al (2005) propose a measurement scale for organizational learning capability, drawing upon a number of other authors who have generated works on organizational learning, leadership and systems thinking. Managerial commitment, systems perspective, openness, experimentation and knowledge transfer and integration are the four characteristics identified as ascertaining an organization's 'sustainable competitive advantage' through linking performance and learning. It has been suggested, however, that using the term 'learning' in relation to organizations, create pedagogical difficulties in attempts to provide empirical evidence about specific business practices that contribute to growth and sustainability (Boreham and Morgan 2004). Boreham and Morgan (2004) suggest that use of a socio-cultural framework where collective activity is considered a social construct and the imperative for 'relational practices' exists, will more accurately measure capability if "...opening space for the creation of shared meaning, reconstituting power relationships and providing cultural tools to mediate learning" (Boreham and Morgan 2004 p.321) are incorporated into organizational practice.

Other capability measurement models, such as the information and communication technology (ICT) model of organizational innovation and individual creativity (Brennan 2005) or the business innovative capacity (BIC) instrument linking Total Quality Management with innovation (PerdomoOrtiz 2006), also aim at measuring a concept which is slippery and messy. Knowledge as the key resource for organizational functioning, where cognition is embedded in practice residing in 
individual and collective consciousness, underpins an organization's capability or 'knowledge advantage' or K-Adv (Walker, Maqsood et al. 2005). Organizational value as advantage in this model, resides in the people infrastructure (human and process capital), the ICT enabling infrastructure and the nature and role of leadership, with competitive advantage being determined by an organisation's ability to provide an "...environment that supports and harnesses knowledge to deliver innovation" (Walker, Wilson et al. 2004 p.3). Knowledge in this sense has been defined as 'latent creative energy' and as advantage is similar to Kodama's (2005) concept of 'dynamic capability' where innovation underpins the concept of strategic knowledge creation incorporating 'networked knowledge communities'. Future corporate sustainability will supposedly depend upon dynamic positioning and capability upgrades through knowledge creation, integration and assimilation, and whilst these strategic approaches will involve processes to integrate internal and external company knowledge for product and service output (Kodama 2006) the challenge is to accurately measure capacity and capability, given the embedded, tacit, subjective, ambiguous nature of knowledge per se.

Resilience of an organization as a socially complex living system assumes a capacity to detect perturbations or changes in the environment, with adaptive processes in place to facilitate embodied cognition for learning (Folke 2006). A resilient organization can sense, interpret and survive major environmental change, can adjust and change practice and creatively utilize opportunities through active social learning involving mutual processes of becoming aware (Wenger 1999; Scharmer and Varela 2000; Folke 2006). The project organization whereby projects as human activity systems act as conduits for organizational change, assume the embedded nature of cognition as inseparable from context. Communal interactions provide an opportunity for 'assimilated meaning' (Freeman 1999) and learning takes place through communities of players intersubjectively connected or coupled with one another within a context (Wenger 1999; Fischer and Ostwald 2001; Barber 2002). Becoming aware, sensemaking and interpretation reside in the individual realm of consciousness, yet collective consciousness as socially mitigated has the potential to support innovation and diversity in organizational response and will have more favourable outcomes when relationships are based on trust (Meyer 1997; Weick 2001; Peansupap 2005; Renzl 2008).

Organisational capacity to adapt, utilising project practice to facilitate structural change, will determine the longevity of system survival. If changes or perturbations in the environment outweigh the response capabilities necessary for self-regulation, then the outcome will be project failure from inability to constrain the organisational 'internal disturbances' and system 'dis-integration' (Hall 2005). The following subsection gives consideration to how 'Quality' both as a concept and a process relate to how a project organization may respond to disturbances in social contexts for system sustainability (Bass and Avolio 1994; Cavaleri, Seivert et al. 2005; Montes 2005; Perdomo-Ortiz 
2006), through utilizing knowledge as the key resource for moving beyond survival (De Geus 1997; Bhatt 2000; Lee 2005) to an emergent, resilient entity.

\subsubsection{Quality, effectiveness and the resilient organization}

This research thesis was instigated from challenges faced in attempting to implement an organizational Quality Assurance (QA) program. The following subsection gives consideration to quality and innovation for effectiveness and emergent organizational change, acknowledging the centrality and embedded nature of cognition, where knowledge as pragmatic may be seen to underpin the flow of action for organizational resilience.

The resilience of complex adaptive systems has been informed by the work of Holling (1973), and as a characteristic of living systems is defined as "...the capacity of a system to absorb disturbance and re-organize while undergoing change so as to still retain essentially the same function, structure, identity and feedbacks" (Folke 2006 p.259). Organizational resilience in a social-ecological context acknowledges the autonomous nature of the organization within a distinct domain and assumes sensemaking activities detect perturbations in the environment with feedback mechanisms facilitating organizational adaptation. An enactivist view of projects as social process, supports the concept of projects as providing the structural openness for organizationally closed systems to selforganize in a state of 'resilient being' whilst incorporating change through learning to facilitate a state of 'transformational becoming'. This transitory state where 'being' is balanced with 'becoming' involves constant emergence necessitating embedded cognition as actionable knowledge being the enabling force. The capacity to tolerate change may be aligned with the resilience of the organization, with effectiveness in functionality correlating with the complementary processes of innovation and continuous improvement. The relationship between organizational cognition and quality processes has been inadequately explored (Choo, Linderman et al. 2007), particularly given the tendency to reify the organizational entity taxonomically with knowledge being objectified as measurable, codifiable, convertible and transferable (Orlikowski 2002). It is suggested that business by and through projects is the innovative actuality of organizations which have the potential to adapt their forms and structures to more ably accommodate for changes in increasingly socially complex environments (Hobbs, Aubry et al. 2008).

Sustainability of an organization in an era of change and complexity necessitates adaptability and sensitivity to shifting environmental dynamics, with variability being underpinned by learning and inquiry. 'Learning to manage by change' has been identified by Folke (2006) as integral for systems moving away from governance by control, with the relationship between organizational learning, 
innovation and effectiveness being identified by Montes (2005) as "...a reflection of an organizations' capacity to fit to their environment" (p. 1160). However, it is felt that resilience is more than "being able to fit' rather, an enactivist view considers that individual members of an organization exist as interconnected embodied beings, whose actions mutually create and respond to environmental factors in a constant cycle of emergent change and adaptation (Orton 1996). As noted by Zeleny (1977), it is the autopoietic nature of the organization that draws upon inputs from the environment for sustainability, then transforms a number of these inputs as outputs, which in turn will impact or influence on the environmental context. This assumes a vulnerability for the organizational entity, with Weick (2001) suggesting resilience to counteract rapid changes comes from at least four sources:

- Improvisation and bricolage - where individuals under pressure can create order from chaos;

- Virtual role systems - where individuals have the ability and permitted space to invent solutions;

- The attitude of wisdom - where individuals have a curiosity and openness to make sense of complex situations, through knowing they don't know or understand everything;

- Respectful interaction - social interaction based on intersubjective relationships where meaning is synthesized through collective consciousness.

Resilience according to Weick (2001) is assumed to exist given the presence of these four factors but may be corrupted in the absence of leadership, clear role delineation, no routines, lack of communication, or most commonly a destabilized structure. 'Structure' in Weick's (2001) model supposes two patterns, the informal shared province of meaning, and the formal structures which constrain such as roles, rules, procedures (p. 116). Enactivist thinking would see these informal patterns in a project organization as involving two or more individuals recursively structurally coupled in language in a project entity, which ably functions to provide the structural openness for the organizationally closed autopoietic institutional entity. Changes in context detected or created recursively, are fed back to this main institution to facilitate any adaptations necessary in the formal patterns of roles and rules for organizational stability. Resilience of a project organization may therefore be seen to depend on acknowledging and managing the complex nature of human interactions both in the project entity and in the organization at large. Multiple interconnecting elements of consciousness resulting from human plurality in an autonomous organization inevitably result in discursive dialogue which adds to the chaos of organizational learning systems (Glass 1996; Cilliers 2000; van Eijnatten 2004). A system's capability of sustaining environmental disturbances, where humans exist in systems of co-ordinations of action, therefore depends upon the cybernetic qualities of homeostatic self-regulation for stability and organizational resilience (Beer 1981). 
Cognition as embodied and autonomous arises from the process of structural coupling, with emergent organizational responses and adaptability being a reflection of a system's competence in its environment (Riegler 2002). This assumes however, an ability of an organization as a complex autonomous entity embedded in context, to self-organise and emerge in a coherent manner (Smith 2005). Consensus domains result from participants being "...involved in an ongoing mutual process of triggering compensating perturbations in one another" (Riegler 2002 p.347). Noting the increasingly heterogeneous nature and complexity of social contexts, this creates challenges for implementing and managing quality improvement programs when ways of knowing and doing may be dissimilar and discursive.

Attempting to manage environmental and contextual disturbances detected does not necessarily fall in the realm of organizational control for maintenance of status quo, rather as noted by Folke (2006), resilient systems utilize disorder as an opportunity for creation and trying new ways of doing. Organizational transformation therefore necessitates knowledge transfer and learning through innovative approaches to practice, with successful change being absorbed into different ways of organizational functionality (Cohen 2001; Clark and Geppert 2006). Quality improvement should be seen to foster enacted cognition, with learning and the creation of knowledge being related "... to how an organization manages the cognitive processes of its members" (Choo, Linderman et al. 2007 p.918). Yet traditionally, quality assurance has been seen as a system of quality control to maintain a certain level of production excellence based on statistical data and logical consequences to meet a variety of different political agendas (Vroeijenstijn 1990; Edgeman and Fraley 2008; Karwatka 2008; Stewart and Waddell 2008). Although Total Quality Management (TQM) has been identified as a “...potential mechanism for managing change..." (Love, Li et al. 2000 p.322), its approach, particularly when overlaid on existing practices, can be rigid, especially with systematic methods of problem solving are applied as in the Shewart's (1931) Plan, Do, Check, Act (PDCA) continuous improvement cycle. Alternatively, viewing a quality assurance project as organizational innovation embedded in context, supports a praxis of emergence where creativity and an increased capacity for flexibility can support organizational adaptation through incremental change (Hobbs, Aubry et al. 2008).

Quality as a system or process may therefore be seen as knowing-in-practice due to its ongoing cyclic nature of embodied action for knowledge creation. The recursive characteristic of organizational change through inquiry, assumes accuracy in interpretation and relevant reciprocal action for effectiveness. Co-emergence of a complex organizational system and its context through structurally coupled interactions, is facilitated by the embedded nature of cognition, where continual structural change may be defined by learning (Maturana and Varela 1998). The enactivist paradigm notes the autopoietic nature of living systems in their ability to adapt and adjust to local conditions (Reid 1996), yet when analysing an organisation's resilience and ability to transform, effectiveness is 
difficult to measure. Mapping of processes, codifying knowledge, studying policies, all serve to present one form of social reality as interpreted by an observer. It is more challenging to map a cognized environment due to the dynamic nature created from multiple and varied interests, values and unique ways of knowing (Aligica 2006).

'Fuzzy cognitive mapping' incorporating a Soft Systems Approach to understanding issues in complex social systems for driving change and promoting innovation has been suggested by Dodouras and James (2007) as having the potential to "...enhance communication, improve the decision-making process and promote sustainable development" (p.850). Whilst other authors have suggested that identifying vested interests and individual priorities involved in innovative, organizational improvement projects may be facilitated by managing risk through the application of stakeholder mapping techniques at the front-end of project implementation (Morgan and Taschereau 1996 June; Brugha and Varvasovszky 2000; Aligica 2006; Walker, Bourne et al. 2008); or via the creation of virtuous circles for bringing about viable change from within (Hilder 2004). The challenge for organisations superimposing structured approaches to process functionality for quality, is balancing systems of measurement with creating the space for creativity and innovation. TQM is a monitoring system that has been designed as a loop to improve performance, but in reality it seems to reinforce instrumentality and impose logic and structure to what should be a creative and risk taking culture if learning for effective change is to be achieved.

As noted by Stewart and Waddell (2008), over the past fifty years of so, Quality Systems have moved from Quality Control of products, to Quality Assurance (QA) of products and processes, to TQM as products, processes and the functionality of the whole organization with Quality Improvement (QI) programs being seen as a process of change that comes from within (Sachs 1994). It is suggested here that 'adding' innovation and creativity to these models will facilitate a social context which recognizes and values the embodied nature of cognition for learning and adaptation so as to move away from more rigid quality systems approaches. Challenges, however, exist in contexts which lack the leadership, resources, motivational work and trust (Amabile 1998; Choo, Linderman et al. 2007) to foster the necessary supportive working environment for creativity and innovation. Organizations that continue to operate with a mind-set of control, may superimpose quality improvement frameworks on operations in the hope for better outcomes, yet these are least likely to facilitate change or improvement due to the lack of stakeholder buy-in and the failure in systems of governance to recognize the importance of cognition as embedded, social situated and enacted. That is, the context per se needs to be 'enabling' for effective knowledge creation and organizational change (Nonaka and Takeuchi 1995; Amabile 1998; von Krogh, Ichijo et al. 2000) if system resilience is to be achieved. 
Despite the difficulties in measuring value-added through innovative practice, there is a recurring theme in the literature linking quality and innovation. Innovation has been noted as a strategy that can make an organization more competitive (Montes 2005); as a dynamic capability which helps an organization modify its behaviour for improved effectiveness (Zollo and Winter 2002); and as the construct of a project organization entity (Hobbs, Aubry et al. 2008). TQM has been identified as a prerequisite to a firm's capability (Perdomo-Ortiz 2006); as the means to create a learning organization (Love, Li et al. 2000); as a mechanism for managing change (CIDA 1995); as being least successful when superimposed on more rigid environments (Jaafari 1996); as a stimulus for discussion and action in striving for a common goal of continuous quality improvement (BurdenLeahy 2005); as a philosophy or mindset (Nichols 2002); and as being most effectively implemented in an organization which fosters learning and knowledge creation through a quality management program (Choo, Linderman et al. 2007). In turn, organisational change has been associated with 'continuous improvement', 'quality improvement', 'quality assurance', 'institutional effectiveness' and 'organisational growth', with 'learning' being identified as the essential factor (Prahalad and Hamel 1990; Sachs 1994; Moingeon 1996; Argyris 1999; Crossan, Lane et al. 1999; Järvinen and Poikela 2005). The nature of this change has been noted as being ontologically prior to the organization, with change being embedded in human action as underpinning emergent states of organizational becoming (Tsoukas and Chia 2002). However, one of the greatest shortfalls that can be seen in attempts to implement TQM systems as a means for creative organisational change, is that "...organizing innovation in a sequential manner lends itself to a generally homogeneous composition of project team members" (Bass and Avolio 1994 p.89). Problems are likely to arise in socially complex environments or when the project team itself comprises a socio-cultural mix of members. Working on stages of TQM rather than implementing quality systems as continual processes that involve all players throughout all stages, creates a system that is likely to stall or head off on different paths, due to miscommunication and misunderstandings between those involved.

Knowledge as being embedded in individuals situated in context, assumes a collective consciousness arising from interrelated realities. Knowledge therefore is changeable yet situation-specific or relevant in context. This has been defined as 'pragmatic knowledge', the "... ultimate knowledge for action - because it is continually being customized and upgraded based on the effectiveness of actions taken in producing the expected results" (Cavaleri, Seivert et al. 2005 p.231). Organizational knowledge may be seen as embodied in and through actions from individuals becoming aware and through social mitigation in context for improved performance and adaptation. This is what underpins innovative practice in organizations utilizing projects as praxis where a pragmatic epistemology assumes enacted cognition. Organizational survival as an evolving state through uncertainty may be seen to depend on projects as innovative processes that facilitate the resilience 
of organisations in the face of socially complex unpatterned events. Although innovation in relation to quality enhanced performance is usually presented as either the exploitation of a company's existing capabilities or the creation of new capabilities (Ambos and Schlegelmilch 2008), it is suggested here that transformational organizational change may be facilitated through project practice as an organizational innovation to facilitate collective consciousness for consensus action. The sensing of perturbations in the social context as an active group seeking behaviour activity (Vygotsky 1978; Argyris 1999; Hall and Paradice 2005; Jerez-Gomez, Cespedes-Lorente et al. 2005) is coupled with innovative creative behaviour that contributes to social change. The project entity provides the structural openness for organizationally closed institutions, to change their form, shift from a period of instability to stability, in a perpetual state of being balanced with becoming through a pragmatism of collective action (Hargrave and Van de Ven 2006).

It is therefore surmised that pragmatic knowledge underpins effective and rapid decision making to move the organizational entity beyond being in a state of learning to that of inquiry for organizational transformation and resilience. Factors that delineate the 'inquiring organization' from the 'learning organization' assume active ways of knowing infiltrate the whole organization via structural artefacts through sensing, interpreting and creating the environment (Courtney, Chae et al. 2000; Cavaleri and Reed 2001; Cavaleri, Seivert et al. 2005; Hall and Paradice 2005), as opposed to the more introspective processes associated with learning organisations. Inquiry, therefore, whilst still being inherent to learning, does add another dimension to the concept of 'productive learning' resulting from single and double-loop learning (Argyris and Schön 1996). The 'inquiring' organisation doesn't only 'modify' its behavior to suit a changing context, rather it contributes to the change through innovative proactive approaches and 'triple-loop' learning (Hall and Paradice 2005). The project organisation may be seen to be the 'inquiring' organization, enabling an emergent flexible approach to business practice through balancing sensing and transformational action for change and adaptation. Such organizations can move beyond the tendency to routinize innovative practice which can cause organizational stagnation (Bartunek, Trullen et al. 2007), to being in a constant state of adaptation and transformation for long term resilience, where the project may be seen as the object of organizational innovation (Hobbs, Aubry et al. 2008).

Although the portrayal of organizations as having the ability to inquire helps in the visualization of movement and change, enactivism moves thinking away from reifying the organization as a conscious entity, to focus more on the embedded nature of cognition and associated consciousness of individual actors within an organization. Deming (1994) presented his System of Profound Knowledge (SPK), with four interconnecting parts:

1. Appreciation for a system - a network of components working together to achieve a common aim; 
2. Knowledge about variation - different approaches to achieve better results;

3. Steps for increasing knowledge - plan, do, check, act (PDCA);

4. Knowledge of psychology - to understand how and why people act and interact.

Whilst these are relevant to organizational transformation, the emphasis in application of quality management systems has tended to be in the use of the third element (PDCA), without giving consideration to the other equally as important and interrelated elements. Deming made reference to a system not being able to understand itself due to 'it' lacking consciousness, rather noting that awareness resided in individual consciousness and the interconnect of the same. Yet the PDCA cycle as a model of quality assurance has been applied at random to organizations globally, without due attention to appreciating the complexity of systems, the impact variations in approaches may have, or in understanding the critical human factor in implementation of quality systems projects. In an attempt to expand on the pivotal nature of intersubjective relationships in organizational transformation, Edgeman and Fraley (2008) have expanded upon Deming's SPK theory to propose a System of Profound Consciousness (SPC). This theory emphasises the benefits of a collective consciousness for the emergence of "...profoundly conscious organizations" which in turn interconnect to create a complex world as a "consciousness supply chain" (p. 686). Through leveraging the diversity in human plurality where interconnected individual realities form a complex multiple whole, 'profound consciousness' may be enabled for transforming organizations into effective thus resilient organizations. System entropy as the 'degree of system disorder or uncertainty' (Edgeman and Fraley 2008) may be counteracted through inquiry for sensemaking, knowledge creation and innovative practice, incorporating collaborative and co-operative behaviour as collective action for emergent states (Van de Ven 2005; Hargrave and Van de Ven 2006).

The enacted organizational environment, where cognition is embedded in context, may therefore be seen as a constant flux of action (Orton 1996), where maturity in technical process merges with maturity in human practitioners to create an organizational "... culture that either promotes good project management practice, or that inhibits it" (Cooke-Davies and Arzymanow 2003 p. 472). The evolution of the resilient organization is one which is able to adapt and change whilst balancing stability and instability in the face of increasing global complexity. Project practice as embedded in organizational actuality creates the structural openness for closed organizational systems to work with rapid change with productivity, quality and innovation connecting in a spirals of performance improvement activities as a means to achieve organization transformation (Dervitsiotis 2008). Organisational adaptation needs to move beyond cyclic improvement processes that leave little space for innovation and creativity, to valuing the benefits from collective consciousness and the role of human agency in ensuring resilience of the organizational entity. 


\subsection{The complexity of context}

Aligning social systems with nature where disorder and uncertainty prevail, is increasingly being considered as a more useful model of social analysis than traditional mechanistic scientific modes that attempt to rationalize and predict behaviour (von Glasersfeld 1979; Varela, Thompson et al. 1991; Reid 1996; 1997; Maturana 1999; Kay 2001; Riegler 2002; Smith 2005; Limone and Bastias 2006; Parboteeah and Jackson 2007). "The world ...appears as a complicated tissue of events, in which connections of different kinds alternate or overlap or combine and thereby determine the texture of the whole" (Heisenberg in Capra 1997 p.30). Over the past ten years or so, 'complexity' in the literature has been increasingly applied in the operational analyses of formal and informal organizations. It has been suggested that through application of complexity theory, organizations may be viewed as Complex Adaptive Systems (CAS), where innovative practice can balance organizational stability with chaos (Law and Urry 2004; Carlisle and McMillan 2006); methods of management can be more effective and relevant (Glass 1996; Cilliers 2000; Richardson 2008); organizations can be better understood structurally (Cilliers 2000; Allen 2006); or functionally (Stackman, Henderson et al. 2006); and through applying chaordic systems thinking (CST) to the connect between chaos and order, structures and patterns which shape human interactions in organizational performance can be comprehended (van Eijnatten 2004).

The follow sub-sections give consideration to the biological nature of social complexity and the relevance to project organization complexity, through exploring:

i. the embedded nature of human cognition and the influence of language

ii. the realities of culture and religion contributing to human plurality

iii. the interconnect of knowledge with politics and power, and

iv. the nature of projects as complex human activity systems

As noted by Boddy and Paton (2004), the three elements of culture, structure and power distribution have been identified as critical factors in relation to project management, and it is suggested that acknowledging the heterogeneous nature of human capital residing in today's organizations is the first step towards successful project implementation.

\subsubsection{The biological nature of social complexity}

Although characteristics of Complex Adaptive Systems (CAS) have been identified by several authors, it is John Holland from the Santa Fe Institute in the US who has been attributed with defining such 
systems. He has referred to CAS as being a dynamic network of many agents interacting jointly and in parallel, where the control is dispersed and decentralized and the system's behaviour is determined by individual decision making and coherence a result of competition and cooperation (Waldrop 1992). Kevin Dooley (1996) also has suggested that CAS comprise agents as evolving semiautonomous units who scan, interpret and respond to an unpredictable and changing environment in a competitive manner for survival. These agents are able to both instigate schematic change and interact with other agents exchanging information in nonlinear flows, having determined rules of interaction both internal and external to the system.

These definitions have been informed by earlier biological cybernetics works of Maturana and Varela (1980), whose autopoietic theory includes descriptions about complex behaviours and relationships that autonomous organisms have both internally and externally through structural coupling of their systems with environments in which dynamic relationships underpin a multitude of interactions. The theory of autopoiesis as a form of analysis in relation to the complexity of socially constructed organizations and their functionality, has been identified as being pertinent to organizational knowledge creation and management, particularly in relation to the biological concepts of enacted consciousness and embodied cognition (Baerveldt and Verheggen 1999; Kay 2001; Riegler 2002; Hall 2005; Buchinger 2006; Limone and Bastias 2006; Parboteeah and Jackson 2007). Evolutionary change as a biological process as applied to organizational adaptation has its origins also in the works of Kauffman and Levin (1987), who note the element of conflict in and between components of complex systems.

“... many parts and processes must become coordinated to achieve some measure of overall success, but conflicting requirements due to alternative simultaneous optimization goals, or conflicting constraints due to the natures of the different parts and processes to be co-coordinated, limit the end result achieved" (p.12).

Adaptation here is seen as more than predicting elements of future change, rather is seen as incremental responses and mutations to previous environmental contexts balanced with 'local search' procedures seeking contextual perturbations (Kauffman and Levin 1987).

CAS are renowned for their instability, emergent and self-organizing properties, with the 'whole' being irreducible to parts and multiple elements of time and space existing. They involve non-linear systems with unpredictable outcomes, irreversible processes and positive feedback loops to reinforce change (Cilliers 2000; Law and Urry 2004; van Eijnatten 2004). Such 'chaos', in which certainty can only be found in uncertainty, is the social reality of an enacted globally complex world, where individual realities are more accurately portrayed as existing in a domain of constitutive ontology entailing multiple realities of probable validity. CAS "...constantly seek to adapt to the 
environmental circumstances in which they find themselves" (Carlisle and McMillan 2006 p.4) with interrelationships facilitating a reciprocity of creation and adaptation through active innovative behavior to move learning beyond maintaining stability to shaping change through inquiry for resilience.

It is the network of interrelationships of a system's components which are integral to both CAS and autopoiesis. CAS entail “...complex structures and patterns of interaction [which] arise from disorder through simple but powerful rules that guide change" (Folke 2006 p.257) whilst autopoiesis is an organization of processes which “... a) recursively participate through their interactions in the generation and realization of the network of processes of production of components which produced them; and b) constitute this network of processes of production of components as a unity in the space in which they (the components) exist by realizing its boundaries" (Maturana 1999 p.153). The constancy of maintaining their own organization through homeostatic systems in essence means such organizations are considered 'organizationally closed', yet it is implicit in this theory that the autopoietic entity exists in a medium with which it interacts and which the interchange of elements with the environment can be observed (Maturana 1999). This is the structural openness that has metaphorically been applied to social organizations as autopoietic entities embedded in environmental contexts where cognition as the 'knowing' is seen as enacted and emerging through engagement with a world. The circular nature of autonomous living systems defined by the nature of structurally coupled interactions as embedded in context is essential for its survival (Maturana 1980).

The resilience of an organization therefore as a CAS may be measured by the capacity to absorb change and shape change through sensing and adapting to disturbances in the environment (Folke 2006) through relationships involving co-emergence and mutual reciprocity. This biological representation of cognition where the 'knower' is a "... complex agent that is organizing, selfmaintaining and structurally determined" (Proulx 2008 p.12) portrays knowledge as enacted at an 'interobjective' juncture where meaning is brought forth through constant evolving interactions. It is through recognizing cognition as a process of the living that organizational responsiveness as a CAS may be seen to reflect the system's capacity to adapt as an emergent entity for transformation and resilience, as opposed to a more constructivist view of system stability involving simpler feedback mechanisms where a state of equilibrium is maintained after a temporary disturbance (Holling 1973).

The main factors which have been noted therefore as characterizing CAS as autopoietic entities include, self-organizing behaviour, structural openness, fractals of other entities, agents interconnected in dynamic interdependent relationships, non-linear processes, patterned dissipative behaviour, emergent entities, positive feedback loops, instability, the whole being irreducible to parts and behaviour which is unpredictable and spontaneous in an environment equally uncertain 
(Law and Urry 2004; Bloch 2005; Stackman, Henderson et al. 2006). Complexity pertaining to organizational systems has been referred to as 'chaotic' with the environmental context being so unpredictable that traditional linear systems of management are considered inadequate in sustaining the organisation (Glass 1996). Van Eijnatten (2004), has suggested that this chaos which goes hand in hand with complexity, can be more ably managed by applying 'chaordic systems thinking' to organizational functioning by utilising five inherent organizational elements of consciousness, connectivity, indeterminacy, dissipation and emergence to create 'sustainable' becoming systems. Whilst the chaotic nature of business practice has been disputed (Cilliers 2000), with it being suggested that "...chaos theory implies that events have a life and logic of their own; there is limited room for intervention" (Tyler 2005 p.569), it is the concept of non-random emergence where the organization moves to higher levels of complexity and coherence evolving collective vision for shared action which Glass (1996) notes as providing the semblance of order amongst chaos.

Therefore, this emerging, evolving nature of complex adaptive systems, implies novel, unpredictable situations which will necessitate new approaches to organizational theory (Richardson 2008) to help in managing or adapting to unforeseeable uncertainty and complexity that typifies today's dynamic business environment (Sommer and Loch 2004). An emergent ontology of becoming is supported by an enactivist paradigm where postmodern thinking substitutes the concept of organizations as static entities with the view of them being cognitive, transient, non-static and proximal and as "...effects of complex social processes" (Chia 1995 p.581). Parallel to calls for a re-think on traditional models of organizational analyses, is an increase in managing business through projects. This in turn has created an imperative to broaden the conceptual framework for project management research (Söderlund 2004a; Whitty 2005; Winter, Smith et al. 2006) and project management theory (Shenhar 1998; Turner 1999; Clegg, Pitsis et al. 2006), to facilitate the management of project complexity in an era of uncertainty (Sommer and Loch 2004; Atkinson, Crawford et al. 2006; Ivory, Alderman et al. 2006; Cooke-Davies, Cicmil et al. 2007; Pundir, Ganapathy et al. 2007; Whitty and Maylor 2007).

Discussions on 'complexity' in project management have predominantly been in relation to solving of organizational problems which are messy structurally and technically, where goals and project specifications are unclear and elements of performance are convoluted (Williams 2002; Atkinson, Crawford et al. 2006; Pundir, Ganapathy et al. 2007). The lifecycle model of projects and project management has dominated project management theory, with the need to increase consideration of the complexity in projects to inform practice, being identified by Winter, Smith et al (2006). The literature seems to indicate that there has been little exploration of project 'complexity' resulting from heterogeneity caused by human plurality both within project teams and project organizations (Cooke-Davies, Cicmil et al. 2007). More recently, it has been suggested that projects (as organizations) may be considered as complex adaptive systems (Cooke-Davies, Cicmil et al. 2007; 
Whitty and Maylor 2007). If we then presume project actuality as a complex social process, then the phenomena of 'unorganized complexity' of this entity will entail outcomes which can only be patterned predictions rather than precise (von Hayek 1974). Divergent individual interactions from differences in language, culture and cognition contribute to the inherent messiness and ambiguity of today's socio-economic environment (Campos and Sanchez 2003), creating a futility when attempting to prescribe specific strategic management approaches to emergent unknown entities.

\subsubsection{Human embeddedness and the influence of language}

The embedded nature of human cognition moves beyond the scientific explanations that are proffered to explain the world from an observer's standpoint, to a view of human social systems as complex systems "...constituted as systems of co-ordinations of actions in language" (Maturana 1988 p.78). The enactivist view of cognition therefore supports a reciprocal interconnect between the knower and the known, where emergent realities may be seen to reside in a domain of constitutive ontology with meaning arising from 'bringing forth of a world' (Maturana 1988; Capra 1997; Proulx 2008) and human action is influential in changing the world. Patterns of meaning from embodied knowledge replace any concept of an 'ultimate' objective truth, so that knowledge is seen as embedded in individuals, emergent in context, uncertain, imperfect and unpredictable (von Hayek 1974). Cognition is therefore a process inseparable from system embodiment, producing meaning through mutual structurally coupled relationships between humans as individual autonomous higher order cognitive entities and their environmental contexts. Enactivism assumes therefore that social interaction can only take place between autonomous systems (Baerveldt and Verheggen 1999) with human embeddedness as a social phenomena being inseparable from the experience of individual participants (Maturana 1980).

Enactivism puts this 'experience' at the centre of cognition, with language facilitating the social interaction which is necessary for accessing the experience of others. Language may be seen as a biological phenomena, where the embedded nature of humans in language determine the praxis of living with 'languaging' evolving from structural recursive interactions of living systems (Maturana 1988). If we assume that culture is symbolically represented in and through language where humans exist in language (Maturana 1995), then the understanding of each other in meaningful relationships, will necessitate a domain of consensus interactions which are a co-ordination of behaviour rather than a system of information transmission. Humans may therefore be seen as immersed in language as a process of making sense of the world in which they are embedded, where cognition as the knowing how is enacted as a complex dynamic system which reacts in a reciprocal manner in changing contexts (Smith 2005). As suggested by Reid (1996), 'knowing is being is doing', with 
meaningful interpretation of the world in which we exist, being an emergent entity as knowledge is continually enacted and co-created through networks of social interactions (Orlikowski 2002; Proulx 2008).

Knowledge as emergent from participation in a world, moves beyond the constructivist tendency to reify knowledge as 'out there' where discourse is portrayed as 'structured collections of meaningful texts' which can be stored and referred to for sensemaking (Phillips, Lawrence et al. 2004). Language as a social 'sensemaking process' involves mutual retrospective interpretation of actions together with proactive and reactive behaviour in relation to the environment (Weick 2001), to conceptually move the human entity and context closer to enacted knowing. Cognition as the 'knowing' for sensemaking through embodied action, is more accurately portrayed as process where co-emerging realities are facilitated by dialogue comprising inseparable elements of emotioning and language in networks of conversations (Maturana 1998). Given our current era of increasing social complexity where diverse social groups interconnect on a global and local scale, these 'networks' could be seen to represent a perpetual reality of intertwined order and chaos. This state has been referred to as 'liquid modernity' due to its unpredictable and irreversible nature (Law and Urry 2004), as a 'constellation of people and things' (Healy 2003), as an 'ensemble of autopoietic systems' of actors and society (Buchinger 2006), which together create an "...insecurity and ambiguity of social life where no fixed solutions exist" (Höijer, Lidskog et al. 2006 p.354).

Organisational human plurality is the new reality where ambiguities result from a clash of internal and external social realities, where emergent entities comprise coupled relationships of individuals from diverse cultural backgrounds. Multidimensional dilemmas are created from the discursive mix of knowledge resulting from the embedded nature of cultural and structural elements at individual, group and institutional levels (Crossan, Lane et al. 1999; Höijer, Lidskog et al. 2006). Such 'epistemological pluralism' (Healy 2003) supports the notion of human cognition as an emerging complex system (Smith 2005) through acknowledging the dynamics of difference that are embodied in systems we may never accurately be able to understand (Riegler 2002).

Reality therefore is relational "...produced and stabilized in interaction that is simultaneously material and social..." (Law and Urry 2004 p.395), not objectively coherent, rather exists as an planation of experience through language (Maturana 1988). This domain is where conflicts arise, due to differences in interpretation of experiences that have been constituted through reflections as an 'observer'.

'Becoming Aware' through a process of suspension, re-direction and letting-go as noted earlier in Figure 9 (Scharmer and Varela 2000) is an individual experience where understanding emerges from subsequent social mediation. Language or dialogue as 'communicative action' moves understanding 
beyond the individual consciousness to an emerging collective consciousness which has been represented as involving stages of reception, interpretation, assumption, conclusion (van Eijnatten 2004) or information, utterance, understanding (Luhmann 1995) or as a stream relating 'psychic' acts to concrete objects (Husserl 1992 in Buchinger 2006). As Varela in his conversation with Scharmer (2000) states, the "...know-what is only used to get started, but it is the know-how that counts and that is going to be transformative" (p.9). It is therefore dialogue through human exchange which may be seen to facilitate collective consciousness and understanding to form the foundation of emergent sensemaking activities. Whilst it has been suggested that this 'understanding' is a result of an intersubjective mutuality (Habermas 1981) which facilitates communicative action through using language and democratic processes to reach agreement, this pragmatic approach tends to reinforce the concept of knowledge as rational and does not easily account for discursive dialogue which is consequential to socially complex situations. Enactivism assumes context is inseparable from cognition as embedded in language. Living systems through being structurally coupled with other living systems are in turn seen to be recursively structurally coupled with language, with selfconsciousness and awareness preceding socially mitigated collective consciousness (Maturana 1999). This creates a 'consensual domain' based on homeostatic activities that are perpetuated through interconnected structural elements.

Language as an emergent social reality thus becomes more complex as a means of processing information when individuals from a melting-pot of cultures comprise a project team, project organization or environmental context. Challenges result when there is divergent 'mental maps' as represented by language, co-existing within organizations (Whitty 2005). Language, in turn, shapes these culturally derived mental maps influencing the flow of consciousness and complicating the understanding process. The uncertainties that exist in a business world of flux and change are made even more ambiguous when stakeholders arrive at the project table with divergent views and understandings. Culturally derived differences will add to the 'competing narratives' (Boddy and Paton 2004) or 'multilogues' (van Eijnatten 2004) that can contribute to project complexity, yet as noted by Healy (2003), encouraging difference through acknowledging epistemological pluralism “...all relevant knowledge, perspectives and viewpoints are employed" (p.700) to facilitate institutional change through reconceptualising knowledge as being multifaceted and emergent.

Consensus in understanding is a concept that is based on the modernist views of an 'ultimate truth', where reasoning and objectivity in knowledge underpin the concepts of transportable information with systems defined according to human projections (Cooper and Burrell 1988). On the other hand, post modern discourse presupposes that consensus through dialogue for mutual understanding is an elusive concept, "... a horizon that is never reached" (Lyotard 1984 p.61), where difference is the glue which is intrinsic to all social action (Derrida 1973). Enactivism as a theory of cognition sits at the 
postmodern end of the philosophical spectrum, supporting the concept of organizations being selfreferential and autonomous complex systems which cannot be reified as entities comprising unified human rationalities. Reciprocity in communication may be seen to facilitate understanding which is emergent, but given the divergent nature of individual lifeworlds in a socially complex environment, it is inevitable that discursive dialogue, misunderstandings and ambiguities in interpretation will result. The embedded nature of difference, as reproduced through culture (DiTomaso and Hooijberg 1996), reinforces the uncertainty of knowledge, where consensus should always be prepared for other interpretations and understanding will involve infinite dialogue (Sikka 2008).

Co-emerging systems, where the social and the environment mutually create and adapt, rely on enacted cognition and communication as elemental processes in reproduction and system resilience. Social complexity assumes discursive relationships which if culturally derived, will also exhibit interrelated issues of control through power and authority as determinants of the nature and legitimacy in patterns of dialogue (Baerveldt and Verheggen 1997; Bourdieu 1998; Sikka 2008). “Discourse transmits and produces power: it reinforces it but also undermines and exposes it, renders it fragile and makes it possible to thwart it" (Foucalt as cited by Edkins 1999 p. 54).

The following two sections therefore give consideration to the realities of culture and religion and the interconnect of knowledge with power and politics as contributing to the social complexity in a project environment. The impact these factors have on communicative processes will inevitably affect emergent understanding, with it being suggested that dialogue may facilitate a collective consciousness, yet consensual understanding will remain elusive.

\subsubsection{The realities of culture and religion}

\section{Culture}

'Culture' has been defined as "...a way of life, a network of meanings, or a system of values and beliefs" (Clarke and Otaky 2006 p. 112); “... a collective programming of the mind which distinguishes the members of one group or category of people from another" (Hofstede 2003 p.5); “... a closed network of conversations which is learned as well as conserved by the children that live in it” (Maturana 1998 Part I p.4); “... shared learned behaviour and meanings that are socially transferred in various life-activity settings for purposes of individual and collective adjustment and adaptation" (Marsella 2005 p.657); and as a form of capital where accumulation of knowledge is associated with the conferring of power and status (Bourdieu 1996). These definitions are all variations upon a theme of some form of identifiable group collectivity, supposing a cultural relativism which determines cultural differences where collectives differentiate from each other in 
meaningful ways (House, Javidan et al. 2002). In various social entities within nation states, Hofstede (2001) has identified two levels of cultural differences. The first level involves values, rituals, heroes and symbols which exist at the social group level and may be considered as subcultures; and the second level overlays cultural differentiation at the national level as measurable dimensions of power distance, individualism and collectivism, masculinity and femininity, uncertainty avoidance and future orientations (House, Javidan et al. 2002; Hofstede 2003). Defining of these dimensions was subsequent to original research by Hofstede (1980) investigating cultural differences amongst various nations, using IBM employees working globally as the main data source. Whilst such cultural variable factors are and have been extremely useful in facilitating an understanding of possible behavioural patterns of particular cultural groups throughout the world, caution is needed in applying rigid models to pre-empt or predict behaviour given that the original study was performed nearly three decades ago. This factor together with the unique nature of emergent cognition in an increasingly global and transient era where cross-cultural interactions are increasingly evident, raises a new reality for business practice.

Cultural relativism assumes that judgment of other human groups based on some form of ethnocentric opinion should be avoided due to the subjective nature of interpreting another's behaviour. "One should think twice before applying the norms of one person, group or society to another" (Hofstede 2003 p.7). Cultural patterning at its worst can contribute to unfounded intolerance of behavioural differences, together with blocking empathetic communication and hindering emergent knowledge for adaptation in an era of uncertainty. "Human survival and well being is now embedded in a complex and interdependent global web of economic, political, social, technical, and environmental events, forces and changes" (Marsella 1998 p.1289). This creates new cultural challenges in an increasingly complex global world where very few if any, nation-states are untouched by forces associated with technological advancements in communication systems, ease of travel, and spread of international business (Robbins 2000; Clarke and Otaky 2006). Societies today are facing situations with an array of complex problems which call for new and emergent solutions which may be at odds with traditional ways of knowing and doing (Höijer, Lidskog et al. 2006).

Given these realities together with recognizing the social nature of human cognition, there is an imperative to move away from viewing 'culture' as a conglomerate of irreconcilable characteristics or as a defined context for human behaviour (Baerveldt and Verheggen 1997). Culture is better presented as an evolving cognitive process where acknowledging difference is the starting point for mutual understanding. Cultural relativism should support a greater tolerance of differences internationally where judgement (and action) is withheld about behavioural differences between nations, and at the extreme, this can mean a non-interventionist approach even if human life issues are involved (Bonney 2004). This is based on the premise that no individual or group should 
condemn another culturally derived behaviour, as there are not ultimate 'transcultural values' that can be used as a benchmark to hold another culture in judgement (Windschuttle 2002).

On the other hand, the concept of cultural differences and diversity (Lingard, Rawolle et al. 2005; Bottery 2006a; Watson 2007) runs parallel to the suggestions that globalisation is creating a greater uniformity in social landscapes caused by the spread of influence from transnational companies creating imperatives which are parochial and universalistic in attitude and predominantly Western business world driven (Bilgin 2006; Clark and Geppert 2006). This together with the spread of English as a universal language of business and highly mobile expatriate workforce, are supposedly making the world a smaller place within an increasingly level socio-cultural playing field (Watson 2007). Growing global similarities in behavioural habits of dress, housing, food, sport and use of technology are examples of superficial changes reflecting a level of international homogeneity, yet cultural differences in nation-states run deeper than the more obvious symbols, rituals and heroes prescribed to by individuals across societies (Hofstede 2003).

Stability or otherwise of cultures may be dependent upon the permeability of a nation to outside influences, with institutions (i.e. social structures, family patterns, educational systems, political systems) varying in degrees of susceptibility (Hofstede 1980) according to levels of maturity and the strength of structurally coupled relationships between elements of the organization and the external environment (Cooke-Davies and Arzymanow 2003). As autonomous systems, these institutions are structured to sense perturbations in the environment which act as triggers to instigate organizational change through cognitive processes finding meaning from a world of experience. Incremental mutual adaptation is the necessary consequence for resilience, with it being possible that shifts in national values and social norms may emerge through individual experiences connected with elements of new and different lifeworlds. Organizational culture can no longer be assumed to represent or mirror a country's national culture despite it being a social system in context. Being born into a specific national culture does not involve individual choice, but belonging to a business organization is usually a consequence of individual decision making, with the subsequent social 'mix' of workers often being in contrast to the external environment (Hofstede 2003).

Culture may therefore be better conceptualized as an emergent cognitive process with social interaction and experience facilitating knowledge development and flow. Communication is integral to emergent understanding, with it being suggested by Han-Georg Gadamer (1975 in Sikka 2008) that agreement to engage in dialogue needs to precede mutuality in understanding. If this agreement takes place, the end product is supposedly 'consensus', yet given the uniqueness of individual experience, together with social role expectations, resultant behavior may appear consensual in nature, but may in actuality be a result of social expectations to conform (Michelfelder and Palmer 
1989; Sikka 2008). Consensual domains rely on a system which orients actors together cognitively, with an 'objective' reality emerging from consensual experience within language (Maturana 1988; Baerveldt and Verheggen 1999). Through acknowledging the centrality of experience in autonomous systems where cognition is embedded and enacted in a world of unique interpretation, we can move closer to the notion that social interaction is the means for cognitive systems to access each other's experience through relationships based on mutual reciprocity (Baerveldt and Verheggen 1999). Organizations embedded in a cultural context, may be seen to be socially constructed with individuals structurally coupled with each other as autonomous entities, whilst also being structurally coupled with an external environment. Cognition is central to the living system, with flow of information emerging from interconnected individuals experiencing the world.

An organization may therefore be seen as a socially cultural cognitive process which exists as an embodied entity in a wider cultural context. Organizations are frequently portrayed as homogeneous entities comprising particular behaviours and attributes which reflect and are moderated by a nation-state's dominant cultural forces and 'national identity' (House, Javidan et al. 2002; Chuah, Hoffmann et al. 2007). Whilst it would be difficult to deny the existence of a nationstate's dominant cultural collectivity, the forces of globalization seem to be generating more heterogeneous systems of different rationalities, with individuals from disparate cultures now working together in countries far-flung from their country of origin. The resultant pattern of discursive discourse which connect individuals in workplace teams, organizations and communities, is the critical factor determining and underpinning emergent social transformation (Clark and Geppert 2006). "Organizations do not first pre-exist and then create their relationships; they occur in existential gaps which lie beyond knowledgeable discourse" (Cooper and Burrell 1988 p.108); that is they are not reified in an objective sense, rather they are emergent, embedded in context and defined through human discourse.

Enacted knowing may be seen to facilitate a collective organizational consciousness, through perceiving the 'knowing-how' as an enabling process where there are mutual efforts to help others 'know in practice' through emergent and enacted cognition to move beyond the impression that knowledge is embedded and 'stuck' in situations (Orlikowski 2002). The challenge is to facilitate the necessary processes of dialogue for meaning to arise through the flow of conversational networks which constitute culture in organizations when individuals from divergent environmental backgrounds are working together.

If nation-state cultural identities are in a constant state of flux due to the affects of a globalised world, then culture may be better perceived as " ... a never-finished site of competing historical and social discourses, rather than as a received set of beliefs and values" (Clarke and Otaky 2006 p.120). 
The concept of 'cross-cultural' knowledge transfer signifies the acknowledgement that there are cognitive differences determined by social mores, traditions and values in and between nation-states which can impact on business and economic practices. These 'knowledge transfer' models have been discussed in relation to 'stickiness' of knowledge (Szulanski 2003); absorptive capacity of a company (Cohen 2001); cross-border knowledge transfer (Bhagat, Kedia et al. 2002; Javidan, Stahl et al. 2005); cross-cultural interactions in economic practice (Chuah, Hoffmann et al. 2007); transnational institutional building (Clark and Geppert 2006); international joint ventures (Barkema, Shenkar et al. 1997); leading teams in different countries (Javidan, Dorfman et al. 2006); cross-cultural knowledgemanagement (Glisby and Holden 2003); and as affecting cultural configurations of organizational accountability (Gelfand 2004). Research into the 'cross-cultural transfer' of knowledge has identified patterns and dimensions of culturally determined behaviour of individuals, social institutions, nationstates and clusters of nations.

The strength of the literature has been in portraying comparative cultural diversity differentiating national populations on an international scale and the difficulties faced when working in unfamiliar contexts. However, the representations tend to reinforce nation-state cultural homogeneity with linear, unilateral processes of knowledge transfer perpetuating the modernist view of knowledge as a transportable commodity in a predictable world. As noted by Hofstede (2003) research into organizational culture has only partly benefited from applying national cultural dimensions to improve our understanding of organizational practice. Perceiving culture as a cognitive process perhaps more ably shifts such symmetrical thinking to a more accurate view of the world as complex and emerging, with social transformation being emergent from individuals embedded in a heterogeneous, increasingly complex world. Human consciousness as embedded in “..people's brains and mental activities simultaneously..." (Jashapara 2007 p.762) supports the concept of individuals, locally, organizationally, nationally and internationally interacting and co-emerging to mutually create context and culture as a collective activity. Increased global human mobility will necessitate empathetic appreciation of national cultural differences particularly when being immersed in contexts of divergent characteristics. But it is suggested that the collective consciousness that arises from relationships of mutual reciprocity will move beyond demands for 'foreign' or expatriate works to adapt to local culture (Gelfand 2004), to situations of co-creation and transformation of cultural contexts, where the domain of language facilitates emergent consensual co-ordinations of action.

Dialogue, as language, involving words, texts and other forms of communication, will inevitably influence how we perceive and understand the world around us (Cooke-Davies, Cicmil et al. 2007). Differences in native language, where language is seen as action and inseparable from cognition, adds another layer of complexity to organizational practice when social interactions of individuals 
involve culturally diverse interpretations of meaningful events. If collective consciousness is situated in context, and that context assumes a degree of historicism and sharing of symbols, values and rituals, then it is to be expected that ambiguity and discursive understanding will result when individuals from disparate backgrounds work and interact. As suggested by Maturana (1998), it is not reason that determines our cultural domain, rather it is our emotions that determine the course of our living domain and thus cultural history. Humans as participating in different communities constituting different emotions and networks of conversations thus all affect "...each other through the intersection of their realizations in our body hoods..." (Maturana 1988 p.25). Epistemological plurality is today's cultural reality (Healy 2003) with diversity in viewpoints and shifting cultures being suggested as more influential in social change today than scientific rationality (Saha 2000) - the challenge is to facilitate a 'meeting of minds' through mutual reciprocity in relationships, for sensemaking to emerge despite barriers that arise from social disparities.

\section{Religion}

Social analyses pertaining to emerging 'multicultural' and diverse socially mixed workforces is found to be lacking in the literature. Rather, the majority of cross-cultural research originating from the Western world tends to focus on knowledge transfer systems and leadership styles which are based upon experiences in reasonably static and homogenous societies (Moghadam and Assar 2008; Muczyk and Holt 2008) or focus on implications for western (usually American) executives working with employees from different cultures (Javidan, Dorfman et al. 2006). Eastern and Middle Eastern derived literature is certainly less prolific, leaving the field of organizational analyses open to cultural appraisals that may not reflect the accuracies of social contexts which are increasingly diverse and subject to forms of contrasting governance (Orlikowski 2002; Bilgin 2006). Although differences in culture have been associated with variations in gender, region, generation, class and religion (Hofstede 2003), it is the latter issue of religion which will be raised briefly in this section due to the presupposed significance that belief systems have on communication and understanding in socially complex working environments. According to Kriger and Seng (2005), who proposed a leadership contingency model based on five major world religions traditions (Islam, Christianity, Judaism, Hinduism and Buddhism), $82 \%$ of the global population believe or follow some form of religion or spiritual tradition. Whilst their research is focused on expanding the theoretical base of organizational leadership, they do note the importance of the social construction of reality and the emerging realities that exist in organizational contexts where potential conflicts arise from discontinuity between organizational values and individual belief systems.

Given the gaps in the literature on the influence of religion in the workplace, drawing upon the literature on organizational behaviour and leadership involving cultural dimensions can provide some distinctions. The Global Leadership and Organizational Behavior Effectiveness (GLOBE) research 
program for example, (House, Javidan et al. 2002) builds upon Hofstede's (1980) work to explore cultural differences between nations and organisations. Despite religion being raised as a 'cultural characteristic' relevant in a number of Hofstede's 'cultural dimensions', the GLOBE study involving 61 nations, does not highlight religion as specifically relevant to organizational practice. The influence of religion in the workplace has been identified as an area under-researched and missing from most organisational literature (Kriger and Seng 2005), despite the complex ontological and epistemological issues beliefs systems can raise particularly in the work place.

Cultural traditions are frequently confused with religious practices, and whilst there is usually some connect between religious beliefs and a country's national political practices and systems of governance, a clear delineation between religion and culture exists. World religions are recognized as being globally heterogeneous and not geographically confined to local contexts, bound by nationstate boundaries or political borders (Hofstede 2003; Bonney 2004; Kriger and Seng 2005). National cultures comprise diverse sub-cultures where meaning ascribed to human existence is disparate. Religions however, or at least the three main western derived religions of Judaism, Christianity and Islam, speak of an ultimate truth or road to salvation with members of the different 'groups' believing that they are the only ones who have the answer to finding that truth (Hofstede 2003). "Religion helps in acceptance of the uncertainties one cannot defend oneself against, and some religions offer the ultimate certainty of a life after death or a victory over one's opponents" (Hofstede 2003 p.110). Hofstede's research notes a positive correlation between individuals in countries exhibiting strong uncertainty avoidance behaviour, with those who identified an affiliation with one of the three more dominant religions (Hofstede 2003); whilst in contrast, individuals belonging to Eastern religions (or Spiritualities) such as Buddhism or Hinduism, were found to be less concerned with avoiding uncertainty than they were in working on self-improvement and dealing with virtue.

Given the increasing 'unknowns' now and into the future, where complexity and non-linear patterns of knowing dominate, religion as a differential value between cultural groups appears to be one behavioural means of reducing anxiety. Religion as a value or ritual may be seen to promote group collectivity to provide a sense of belonging thus improving coping mechanisms to deal with rapid global change. Nations however, who have scored high on the uncertainty avoidance index (UAI) have been associated with cultural groups exhibiting behaviours reflecting a religious fundamentalism indicating an extreme intolerance towards ambiguity and unpredictability (Hofstede 2003; Marsella 2005). A large percentage of these countries also have large power distance ratings, where power distance is defined as "...the extent to which the least powerful members of institutions and organizations within a country expect and accept that power is distributed unequally" (Hofstede 2003 p.28). Power has been suggested as the integrating factor which combines truth, rationality 
and knowledge (Flyvbjerg 2001), as able to free our imagination (Clegg 2006) and as a producer of knowledge (Foucault 1977). Recognising the influence that culture has as a cognitive process in constructing our realities and influencing our interpretation of the world, there is a need to acknowledge the increasingly global presence of discursive plurality involving religious and political differences, to move beyond a tolerance of 'otherness' to a world that utilizes power for integrative and unified solutions through difference.

The religious philosophical roots of Judaism, Christianity and Islam have all been associated with originating in the Middle East region (Hofstede 2003), yet it is Islam which dominates the Arab world of today. This region primarily encompasses the Arabian Peninsula, Northern and Western Africa, Iran and areas of Eastern Africa, and in collective areas within the regions of Central and South Asia, India, Malay Archipelago and other populations in the Balkans, Russia, Europe and China. Followers of the Judeo-Christian religion are also found worldwide, but have been identified as being concentrated mainly in the 'Western world' of the United States of America, Australia, United Kingdom, Europe and Canada. In the project GLOBE research study which aimed to identify cultural differences between international communities while working towards finding explanations and leadership qualities that may be effective (House, Javidan et al. 2002), clusters of countries sharing similar characteristics such as religion, were grouped for investigation, analysis and comparison with other clusters.

The countries of Egypt, Morocco, Turkey, Kuwait and Qatar were selected to represent a Middle Eastern 'Arabic cultural cluster'. However, although the majority of individuals in these populations are Muslim, with the Quran being identified as the 'unifying force' that guides societal practices and creates a commonality in culture (Kabasakal and Bodur 2002), there are historical disparities between each of these countries, particularly in relation to economic performance and source of wealth (Iannaccone 1998; Kuran 2004; Noland 2005). In contrast, the Western world presented as representative 'Anglo-cluster' involving the countries of Australia, Canada, England, Ireland, New Zealand, South Africa (white sample) and United States of America are more geographically dispersed and racially diversified, yet share Judeo-Christian values balanced with a secularity supporting an individualistic performance orientation (Ashkanasy, Trevor-Roberts et al. 2002). Arabic identity is associated both with Islam and with the Arabic language in the Middle eastern cluster (Kabasakal and Bodur 2002). Whereas the Anglo-cluster of countries all share English as their national language but no reference is made specifically about religion as being a defining characteristic of the cluster, rather their pluralistic democracy and egalitarian nature seems to be the glue that binds (Ashkanasy, Trevor-Roberts et al. 2002). 
Religion in the Western or Anglo world may be better perceived at the sub-cultural level of symbols and rituals, where despite political and judicial systems perhaps reflecting generalized understandings of rights and freedoms, the separation of church and state in their systems of governance assume a social religious plurality based on democratic ideals. Current political systems in the Arabic cluster differ according to systems of governance with their binding social factor being Islam. Approximately $90 \%$ of the populations in this cluster are Muslims of the Sunni belief (Kabasakal and Bodur 2002) who base their life on Prophet Muhammad's words and deeds, which in turn forms the basis of Islamic or Shari'a law. As noted by Lawrence (2005) "There are four elements without which law could not be law in an Islamic key: the Qur'an, the Sunnah, consensus and analogy" (p. xiv). The Qur'an has over 6600 verses and 500 of those approximately are noted as having legal content from which 'fatwas' as authoritative legal opinions are drawn. Religion, 'Din', defines the group collectivity, which in turn determines the political and judicial systems, the law (as Shari'a) being unchangeable (Kabasakal and Bodur 2002). With globalization pressuring people in authority in the Arab world to adapt to more democratic and individualistic ways of thinking (Kirat 2005), social and cultural conflicts will inevitably result given the nature of the Islamic legal system. Despite the evolving interpretative arm (figh) of Arabic law and legislation, Shari'a as the dominant system of law draws upon the unchangeable texts of the Qur'an and the Sunnah.

With existing social science theories and research predominantly being developed based on 'Western' experiences involving Western ways of knowing, it has been suggested that in application in 'non-Western' contexts such as the Middle East, theoretical assumptions tend to be parochial and of limited relevance (Marsella 2005; Bilgin 2006; Clegg 2006). The research of Hofstede (Hofstede 1980; Hofstede 2001; Hofstede 2003) and the work from the project GLOBE (House, Javidan et al. 2002; Javidan, Stahl et al. 2005; Javidan, Dorfman et al. 2006) together with numerous other studies drawing upon Hofstede's defined cultural dimensions, has progressed thought in relation to identifying cultural differences of national groups or clusters of countries throughout the world. Yet what is missing is the recognition of the emerging and changing cognitive nature of national cultures in an era of increasing complexity and uncertainty. It is an era where the contemporary merges with traditions of the past, where human plurality in belief systems is at odds with political systems of governance and where parochial superiority in research limits the true global understanding that is needed for new cultures emerging as epistemologically pluralistic (Healy 2003). With culture being portrayed as an emergent cognitive process, where realities have the potential to evolve through mutual interactions, there is a need for an increasing recognition of the influence of culture and religion in determining how we perceive and cope in a non-static dynamic world. Change is inevitable in our global society and learning to accept, move and live with uncertainty and doubt is the new social and political reality which cannot be marginalized (Marsella 2005). 


\subsubsection{The interconnect of knowledge with politics and power}

Epistemological pluralism assumes there are divergent ways of knowing that inevitably create a complexity in context where culture is a cognitive reality, difference is encouraged, and power relations are a political ontology comprising multiple choices (Healy 2003). Social complexity in organizations involving project practice where projects are seen as the source of the complexity rather than solution (Turner 1999; Söderlund 2004a; Ivory, Alderman et al. 2006), moves thinking beyond project uncertainties being defined in terms of estimations, stakeholders and lifecycle problems (Atkinson, Crawford et al. 2006). Perceiving organizations as emergent social cognitive entities where project practice is integral to resilient functionality, highlights the complex interrelationships projects have as autonomous entities coupled with both organizational and environmental contexts. Noting the effect of increasing global mobility on the nature of the human element in organizations, together with the spread of multinational, trans-national business corporations, a new 'complexity of a human kind' comprising a coalescence of multiple realities, discursive cultures and divergent beliefs systems are dominating the actuality of project and organisational praxis. As a socially complex mix, where knowledge emerges through language, change is a constant and sensemaking is culturally conditioned, it seems inevitable that issues of autonomy and control involving power-conflicts will appear both at the institutional and national levels as nation-states are pressured to reposition themselves for an unknown future.

Inequalities in social systems are a reality given the complex nature of interacting components involving discursive relationships and communicative patterns both on a national and international scale. It has been suggested that survival and resilience of these systems as complex and adaptive, may involve tribal behaviour which aims to protect the familiar through the wielding of power as domination (Hilder 2004). Tribalism traditionally served to provide security and social solidarity based on kinship (Issawi 1987) and although elements of such models may still play a major role in how we think and behave today, perpetuating the instrumentality of power as involving relational domination, may in actuality be counterproductive and detrimental to social and thus organizational viability (Hilder 2004). Tribalism assumes there are some in authority, others who follow and a 'glue' which binds, whether that be language, religion or other values, rituals or traditions.

Power as assumed domination of one over another, has influenced traditional social and thus organizational analyses. But given the increasing complexity of today's globalised and interconnected world, calls to reconsider 'power' in relation to globalization suggest patterns of redistribution, emergent relations and disembedding of power relations from local contexts. Where 'maturity' of nations evolve from exposure to a breadth of new realities previously not considered nor experienced. We are experiencing a world which is enacted through a web of connections that link 
local communities from one region in such a way that behavior shaping the local environment can be sensed as perturbations or trigger factors for adaptive change in communities on the other side of the globe (Held, McGrew et al. 1999). Politics has become globalised, with borders of nation-states becoming increasing permeable. State power has not necessarily reduced, nor has tribal behavior to protect national identity. What is being evidenced however, is co-emergent transformations of political systems as adaptive responses to triggers in the global economic environment, with behavior balancing what shapes and contributes to change. We are experiencing a "...growing enmeshment in regional and global orders and the proliferation of transborder problems has created a plurality of diverse and overlapping collectives" (Held, McGrew et al. 1999 p.6). This interconnected complexity implies a more globally dispersed and flattened view of the world, where 'power as influence' as opposed to 'power as domination' seems a more relevant model to support the conceptualization of an emerging complex world (Capra 1997). This world of dynamically related and interconnected lifeworlds, where ontological difference implies a multiplicity which cannot be defined as a single static 'world' (Law and Urry 2004), necessitates power as an enabling force to enmesh individual consciousness within a collective.

Implicit to both the influence of one over another and the domination of one over another is the flow of force involving compliance where power is perceived as a commodity which can be possessed. The difference between the two forces may be an assumed legitimacy in authority of the one holding the power of influence, and perhaps a degree of choice of the one being at the receiving end, with commitment, compliance or resistance being the result (Yukl 2002). However, both aspire to a relational view of power reflecting an emphasis on 'power over' another and serve to maintain an agency-structure divide (Marshall 2006). Various models of power typically present 'power' as a definable entity which can be possessed and is observable in behaviour. French and Raven's (1959) taxonomy of coercive, reward, legitimate, expert, referent power bases (Yukl 2002; Marshall 2006), is one such model which has informed research into power relationships in social contexts and leadership behaviour. Yukl (2002), for instance has incorporated French and Raven's five identified sources of power with work completed by Bass (1960) and Etzioni (1961) who divide power into 'position' or 'personal', to provide a new model of power in organizations. Legitimate, reward, coercive, information and ecological power are presented as derivatives of position power, whilst referent and expert power is associated with personal power. The complex nature of such models assume interactions between the different factors, however objectifying power as an entity of control not only negates the realities of autonomy, but omits the interconnect and variable factors associated within a socio-cultural context. In models where power is associated with a particular source, such as personal or position, the limitations perceived in reifying 'power' as a definable entity, reduces the importance of complexes and interplay of numerous social factors operating 
locally and globally. Today, with nation boundaries between social and economic being considered 'fuzzy' and blurred, and relationships being non-linear, reciprocal and self-perpetuating, predicting the impact of local dynamics on a globalised world and vice versa is an unknown entity (Law and Urry 2004).

Attempts to 'connect' sources of power within organizations for a more integrated approach to analyse some of these global uncertainties are evidenced in the literature. For example, 'episodic' power involving the interplay of numerous stakeholders vying for control within organizations is identified as needing to be balanced with 'systemic' power diffused through social systems embedded in context (Pfeffer 1981; Lawrence, Mauws et al. 2005). Influence and force have been associated with episodic power, and domination and discipline with systemic power (Lawrence, Winn et al. 2001); systemic power has then been divided into 'dispositional' power (rules and norms of social membership) and 'facilitative' power (organizational processes) to link with episodic power to create a power circuit (Clegg 1989). Although such models demonstrate an appreciation of the complexity in organizational functionality and structure, there remains a reliance on the objectification of power thus perpetuating the agency-structure divide.

Chaos and ambiguity intertwine in a complex adaptive world in which social inequalities result from an assumed unequal dispersal of power - whether that is by domination or influence or as an emergent force. It is a reality that is culturally defined, is reflected in values, traditions and behavior, its causation being attributed to economics and a variety of other cultural factors that determine difference and maturity of a nation (Hofstede 1980; Fukuyama 2000; Gelfand 2004; Clegg 2006). Human dominance of one over another involves power. Tribalism reflects one form of social cohesion that may in essence have faded in a pluralistic secularized society, but the interconnect of individuals embedded in an array of socio-political contexts remains a reality, with unequal distribution of power acting as a means to avoid entropy (Hofstede 1980).

Power, also has been defined as a 'measurable dimension' existing in all cultures to a greater or lesser degree as a result of global research investigating cultural differences between nations and cultural groups (Hofstede 1980). A Power Distance Index (PDI) which scales countries from large PDIs to low PDIs has been developed, with power and dependency relationships presented as a reflection of social inequalities. National and organizational PDIs have been investigated, with results suggesting a spectrum of power relationships existing within countries and organizations. Social groups of large power distance ratings having individuals exhibit submissive dependent behavior towards superiors, rarely challenging those in power and in actuality, tend to indicate a preference for autocratic or paternalistic behavior to be demonstrated by those in 'control' (Hofstede 2003). At the other end of the power distance spectrum, low PDI social group individuals are not afraid of their 
superiors, they prefer consultative work styles and indicate they would have difficulties working in an environment which is rigidly bureaucratic with a top-down approach to management (Hofstede 2003). The research concludes that these power distance differences not only reflect social inequalities found to correlate with social class, education and occupation, but are evident of national political systems and ways of thinking and communicating. Culture as 'collective programming of the mind' underpins power as one of several identified dimensions and assumes that the non-random nature of human action provides a framework of social system predictability that is culturally patterned (Hofstede 1980).

This patterning, which may also be seen as a collective consciousness, is similar to Pierre Bourdieu's (1977) 'habitus' which represents a system of transferrable tendencies. His relational approach to social class conceptualization however, defines social inequalities and thus social class distinction, as being reflected in agents competing for control of elements within a 'field' through using their economic, cultural, social and symbolic capital, which is overlaid by a field of power. Bourdieu's cultural-structuralist approach is embedded in social context with relationships between subjects being noted as interconnected, with individual dispositions of social space being relational and constructed from a young age (Bourdieu 1977). The benefits of his work may be seen in encouraging a shift in thought from cultural patterning of social inequalities according to pre-determined socially defined classes which reside within nation-state boundaries as noted in Hofstede's (1980) work, to noting power struggles that may exist between social spaces or fields that are determined by individuals perceiving where they fit in these spaces. The defining elements of the power relationships in both Hofstede's (1980) and Bourdieu's (1977) models are ascribed by the 'less powerful'. However, Bourdieu's social analyses involves social forms of capital which are legitimated through symbolic systems of meaning, thus presenting a framework which can be compared on a global scale. For example, symbolic capital of the 'culturally perceived elite' as a recognized power source, may vary in characteristics globally, but social ascriptions of meaning may be consistent.

The increasingly complex nature of a globalised world has contributed to nation-state identities not always reflecting the gradual differentiation of social institutions, with conflict within the field of power creating new and emerging alliances which may destabilize the social order (Lingard, Rawolle et al. 2005). Power as a defined reality is constructed by the individual perceiving the reality, and this being inseparable from a cultural context, underpins the suggested inequalities existing in relational power definitions. The realities facing nation-states, particularly those who have gained political independence in the last few decades, comprise a need to balance a wealth of available ideas which are as diverse as the world itself, with the need to maintain legitimate power and authority on a national level. Knowledge as an emergent form of capital, is shaping and changing socio-cultural contexts globally, and while this "...knowledge ... embroils us in complexes of both 
people and things..." (Healy 2003 p.693), it needs to be seen as a reciprocal emergent reality influenced by the power of communication. Nietzche's genealogical concept of 'will to know' about the world is based on the postmodern assumption that there is a compelling force which is enacted due to an intolerance of not knowing (Cooper and Burrell 1988).

Discourse needs to become the new reality to step outside preconceived ideas created through conceptualizing power as domination, to facilitate more relevant interpretations of a 'power to' strategically facilitate a 'become aware' of the emergent nature of cognition (Foucault 1977; Cooper and Burrell 1988; Capra 1997; Scharmer 2001). Through suspending self from usual ways of thinking and redirecting reflection to new and emerging realities relevant in the actuality of organizations as networked complex adaptive systems, a more relevant interpretative model which reflects the interconnect between agency and structure as non-static entities will be possible.

Hofstede (2001) in his relational and logic PDI taxonomy, has predicted that despite an increasingly interconnected global world, power distance factors between nations may increase, as small countries become increasingly influenced and dominated by larger transnational companies and supranational organizations. Weakening of the power of nation-states has been presented as a possible consequence of globalization, despite fluctuations in population size and improved access to technology and education, but this 'hyperglobalist' perspective of nation-state fragmentation (Held 1991) assumes a race for global economic sovereignty in a quest for power that may not exist. The premise that smaller countries or those economically poorer will not be able to compete in an era dominated by multinational corporations and regions of free trade (Hofstede 2001), perpetuates a rationalist view of international power relations, rather than considering the transformational influence economic globalization may have on nation-states (Held, McGrew et al. 1999). The perceived limitations that some countries may face in an international market need to be balanced with the realities of an interconnect between economic environments, politics and socio-cultural values (Whitty and Schulz 2007). Nation state politics and national identities are evolving due to the embedded nature of individual countries within a global entity where the flow and dynamic mix of cultures are contributing to the transformation of organizational and national social systems (Held, McGrew et al. 1999). The complexity of power relations globally is making such realities more challenging, with even the most 'powerful' nation-states facing difficulties when attempting to 'control' situations which are changing on a daily basis (Bottery 2006a). Economic globalization is supposedly driving the changing nature of business, with attempts to commodify and market 'knowledge' on a global scale, rather than recognizing the dynamism of cognition as a changing emerging entity which evolves in context. The attempt to manage and control ideas for use has been associated with 'power' of a nation (Bottery 2006a) yet this perspective reinforces a model of human rationality which assumes the world of today is predictable. Universalizing national identities is not 
the reality, rather ambiguity in ways of knowing will escalate, with attempts to hold on to what is familiar being evidence of a tribalism which needs to be accommodated for (Naisbitt 1998) as new and emergent lifeworlds are enacted.

\section{Organizational power}

Discussions on power in relation to organizational theory have traditionally followed positivist models of instrumentality, with linear and causal consequences involving the 'wielding' and receipt of power. Minimal attention has been given to issues of power and politics in organizational management control systems, organizational learning, or as explicit to project organizations or emergent patterned organizational ontologies (Abernathy 2004; Lawrence, Mauws et al. 2005; Clegg 2006; Marshall 2006). The embedded nature of power as relevant to organizational existence, both as an inextricable reality in and between human interrelations, together with systems of governance and the realities of culture, cannot be ignored. Given the centrality of communication, where language as a cognitive process underpins meaningful interpretation, acknowledging the realities of power and politics as situated or embedded within complex projects supports the notion that "...political aspects of the interactions in an organization are not something extraneous to the workings of that organization" (Cilliers 2000 p.30).

As we enter an era where organizational resilience is being recognized as dependent upon acknowledging the centrality of human action in responding to and creating change both within social and environmental contexts, it is crucial that issues of adaptive governance are explored (Folke 2006). As noted by Trevor Hilder (2004), despite innovative and creative practice being encouraged for emergent change and improvements, if such behaviour is perceived as threatening to the 'power elite" within an organization we may find ourselves "...greeted with hostility, which can threaten [our] wealth, health and life" (p. 634).

Social inequalities that exist in organisations as institutions in today's globalised world, may be seen to be perpetuated by systems of control which act in specific ways in defining and determining reality (Hasselbladh and Kallinikos 2000; Phillips, Lawrence et al. 2004). The concept of an organization as emergent and 'spontaneous', moves beyond the reification of an organization as a predictable entity reflecting the social context in which it resides, to seeing organizational systems as having a life of their own that is beyond human definition. Cognition is the human reality in constructing interpretations and contributing to changes in the world around us (Cooper and Burrell 1988), with the embedded nature of knowledge in individuals, in interactions, in context, as enacted through language as discourse, better reflecting the cultural realities of today's dynamic, complex world (Campos and Sanchez 2003). It is language which mutually reflects and determines the social nature of discourse, which may be emergent but socially conditioned by dominant ways of thinking, 
knowing and doing (Chia 1995; Edkins 1999). Discursive practices reflect a complexity in factors influencing communication, where power is implicated through a 'power-knowledge' nexus that serves to sustain control at the expense of autonomy (Foucault 1982). "Discourse transmits and produces power: it reinforces it but also undermines and exposes it, renders it fragile and makes it possible to thwart it" (Foucalt in Edkins 1999 p.54).

\section{Leadership}

Conceptualizing knowledge as truth where "...the power elite within the organization usually determine whose truths count as true" (Tyler 2005 p.568) may be a historical reality but as a model is no longer relevant in an era of dynamic change. Power relationships within organizations as expressed through discourse can pre-determine answers that are based on recognition of those in authority, but these do not necessarily reflect consensus in understanding (Foucault 1982). Normative rational explanations of power in organizations reflect an instrumental control model of the organization where human rationality is preconceived and defined. Whereas an enactivist view of an organization facilitates visualizing an emergent model which is self-perpetuating and autonomous, where governance is non-hierarchical and co-ordinating, and power as a strategic force produces new realities rather than control (Cooper and Burrell 1988; Reid 1996; Capra 1997; Maturana 1999; Buchinger 2006).

Organisations, as are cultures, are better perceived as cognitive processes involving human activity which are mutually reactive and defensive (Cooper and Burrell 1988). The active component is the reciprocal force that contributes to change and adaptation involving patterns of human intervention at local levels (Chia 1995). The conceptualization of power in organizations, as mentioned, has predominantly been in the Weberian tradition of one wielding power over another (Weber 1978), with assumed co-dependent relationships existing, so that those without power behave in a subordinate manner. The conveying of power as an instrument of control is culturally and socially derived, varying in nature according to variations in social values and traditions. Power has also been portrayed in a pluralist sense, residing in conflicts of sectional interests where the most powerful are those who are most successful in achieving their desired ends (Dahl 1957). Power in most definitions is relational and usually dyadic (Baerveldt and Verheggen 1999), whether it be the disempowered being dominated (Tyler 2005), the followers being led (Atwater and Bass 1994; Antonakis 2003; Avolio, Gardner et al. 2004; Cavaleri, Seivert et al. 2005; Gardner, Avolio et al. 2005) or the individual being influenced by another (Shamir and Howell 1999; Krause 2004; Kennedy-McColl and Anderson 2005; Montes 2005).

This is the point of discussion where the role of the leader has entered. The realities of today, moving towards an unknown future, will require leaders as facilitators - to "sort out what's tribal and what's 
universal" (Naisbitt 1998 p.221). Relational models of power have dominated and informed theories of organizational leadership, with definitions presenting leadership as a concept, "...the influential increment over and above mechanical compliance with the routine directives of the organization" (Katz and Kahn 1978 p. 528); as a process, "... the accomplishment of a goal through the direction of human assistants" (Prentice 1961 p.103); as a behaviour, where a person "...commits people to action, who converts followers into leaders, and who may convert leaders into agents of change" (Bennis and Nanus 2003 p.3). Central to each of these definitions is the presence of 'influence' of one party over another to effect change or an outcome. Influence assumes the presence of power, a dynamic energy source which has the potential to be used or abused. The pivotal nature of leadership in organizational change through learning has been associated with legitimacy of influence and authority of a leader to effect change, based on power as ascribed by followers or other stakeholders (Yukl 2002). The inequalities that exist in such relationships are defined by those being 'controlled' rather than those 'wielding' the power, as described by Hofstede's (1980) PDI cultural dimension.

Acknowledging the embedded nature of organizational cognition where the uniqueness of learning arises from experience and interconnections, it is suggested that those in leadership positions are not able to 'manage' experiences or individuals due to the embodied nature of mental models (Campos and Sanchez 2003). Envisaging leadership as 'emergent' as opposed to the 'one who wields power' better reflects the flexibility of roles and the complex responsive patterns of behaviour that are more relevant for new organizational realities. Organizations as complex adaptive systems have dynamic change as a constant, necessitating models of leadership that are flexible, " ... not allocated to a single person, but ... taken up by different persons in a group, initiated at their own discretion" (van Eijnatten 2004 p.442). Knowledge as the cognitive element of organizations may therefore be seen to shape interrelationships for power to be effected (Healy 2003), with autonomy of leadership facilitating the creation of an environment as opposed to control (Waddell 1998).

Power needs to be seen as a producer of knowledge involving different individuals with different rationalities (Foucault 1977), embedded in a context inseparable from sensemaking activities enacted on a global scale. Organisations as comprising socially discursive networks of individuals interpreting the world around them create a multiplicity of social constructions, which can be enabled through the medium of language as discourse. Resilience of an organization as a complex adaptive system will be guided by emergent leadership which can balance individual historical reflection, with a future orientation through establishing power which transcends restraints of control in the pursuit of autonomous institutional change (Healy 2003; Clegg 2006; Folke 2006). 


\subsubsection{Projects as complex human activity systems}

Project management has been viewed using complexity-inspired metaphors (Ivory, Alderman et al. 2006; Cooke-Davies, Cicmil et al. 2007; Pundir, Ganapathy et al. 2007; Richardson 2008) to facilitate a shift in thinking from positivist inspired models of instrumentality and predictability, to conceptualize project practice as entailing dynamic patterned behavior which is self-organising and co-emergent. Multiple interconnections in the project as a social process, involve mutuality in creation and adaptation in environmental contexts. As presented in section of 2.5.1, aligning social systems with characteristics of complexity theory has the potential to focus on the realities of the social interrelatedness in organizational and project practice to unravel some of the unknowns impacting upon project trajectories. It has been identified that project management research is in need of new models involving ontological and epistemological theories to "...illuminate the complexity of projects" (Winter, Smith et al. 2006). The lack of available management tools to 'control' or manage such complexity (Cooke-Davies, Cicmil et al. 2007; Whitty and Maylor 2007) lie alongside calls for further research into social arrangements and patterns of interactions between people, and for further enquiry into power and identity relationships in projects as social processes (Winter, Smith et al. 2006; Cooke-Davies, Cicmil et al. 2007). Conceptual frameworks attempting to explain changes in organizational functionality need to focus more on the individual as the unit of analysis and progress to collective models of activity systems that are networked, community driven and in a constant state of flux (Engeström 2000). Such thinking will contribute to a better understanding of how loosely coupled entities co-emerge and dissolve relationships throughout incremental transformation processes.

The workplace of today is embedded in a globalised emerging context which is culturally diverse and pluralistic in nature. Changes in organizational dynamics where blurring of cultural, national and international borders dominate, necessitates greater consideration be given to the connectedness of action and context for indeterminate emergence of cognition in complex environments (Pettigrew, Woodman et al. 2001b; Orlikowski 2002). The resilience of a project organization may be seen in its ability to move beyond adaptation in context, to create and shape the environment through collective activity, with systems of adaptive governance accommodating for and working with complex and dynamic situations (Folke 2006).

Project management models which align project complexity with structural uncertainty (Williams 1999; Sommer and Loch 2004; Atkinson, Crawford et al. 2006) without giving consideration to the ongoing social interactions creating the dynamism in projects as complex adaptive systems (CAS) (Whitty and Maylor 2007), only in part contribute to understanding the impact an array of discursive social agendas may have on project outcomes. Organizations and teams as communities of practice, 
have been identified as CAS (Carlisle and McMillan 2006; Stackman, Henderson et al. 2006), so that viewing projects in the same light appears a logical step. Dooley's (1996) nominal definition of CAS emphasizes the semi-autonomous nature of complex systems comprising agents who interact with and interpret their environment within rational bounded schema, to change for a 'better fit' with the environment. Flows of information in and between agents and schema are non-linear and may be determined by external agents and the nature of the interconnectedness. If we view projects as complex adaptive systems, the instrumental lifecycle model of projects as tools to manage organizational complexity is replaced by an ecological representation of projects where actors are interconnected with each other and the environment (Ekinsmyth 2002; Söderlund 2004a); numerous influential variables and numbers of interactions are involved (Sommer and Loch 2004); and competing narratives arise from the very nature of complex project praxis (Boddy and Paton 2004).

It is therefore the human element as inherent to projects as social activity systems which implies a complexity due to the individual nature of cognitive elements interacting in numerous and variable ways (Waldrop 1992). The complexity need not assume chaos and disorder, rather should acknowledge the realities of human life as patterned yet discursive and culturally pluralistic. That is, 'organised complexity' where "...the character of the structures showing it depends not only on the properties of the individual elements of which they are composed, and the relative frequency with which they occur, but also on the manner in which the individual elements are connected with each other" (von Hayek 1974 p.3).

Dynamic systems theory, as a mathematical theory, has informed social portrayals of connectivity, where non-linear processes, indeterminate consciousness and 'language as cognition' arise in consensual domains for sensemaking and transformational change. Complexity, uncertainty, contradictions, dilemmas and divergent views dominate late modernity (Höijer, Lidskog et al. 2006). Traditional models of social theory are inadequate in explaining new realities and 'controlling' the consequences, due to complexity resulting from our 'human-made artefacts' and objects of work (Kelly 1998; Engeström 2006). Relationships have now replaced these objects in priority, with the concept of chaotic behavior as unpredictable, yet deterministic and patterned, being drawn from chaos theory involving such entities as strange attractors, fractals and complex trajectories existing in abstract or phase space (Capra 1997).

Living systems as autonomous, self-organising, autopoietic entities recursively regenerating are markedly similar to Henri Poincaré's (1963) mathematical topology of continuous transformations of geometric figures whose properties are constant. Examples in application and the relevance of Poincaré's (1963) topology to chaotic systems theory, are found in the 2-dimensional 'Ueda' strange attractor developed by Yoshisuke Ueda (1993) where a fixed point at the centre of 2-D phase space 
metaphorically 'attracts' the trajectory; and in the 3-D Lorenz attractor designed by Edward Lorenz's mathematical discovery (1963), where each point in the phase space may be seen as complexly connected with the trajectory of the strange attractors which have an ordered pattern which is unpredictable (Figure 10 and Figure 11). The non-linear processes in trajectories which are complex, in spaces where all variables are interconnected, are metaphorically similar to chaotic but orderly human behavior, where the structure of living systems may be seen as the physical embodiment of its pattern of organization and cognition as the process of life being the distinguishing characteristic (Capra 1997).

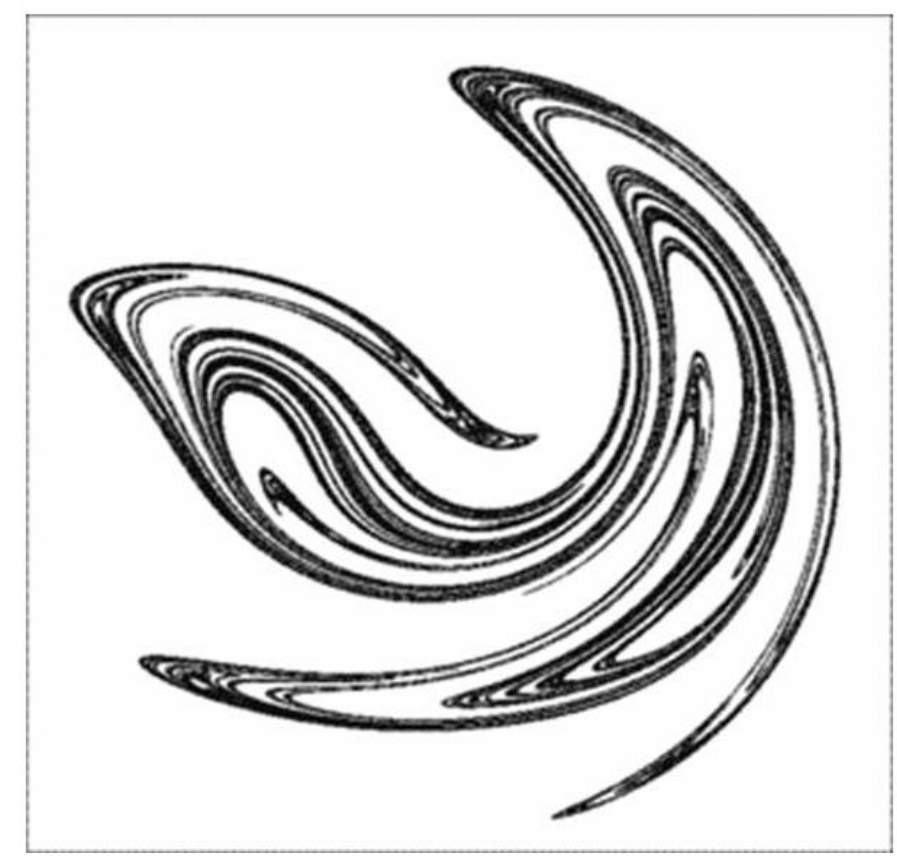

Figure 10 Ueda attractor (Ueda, 1993)

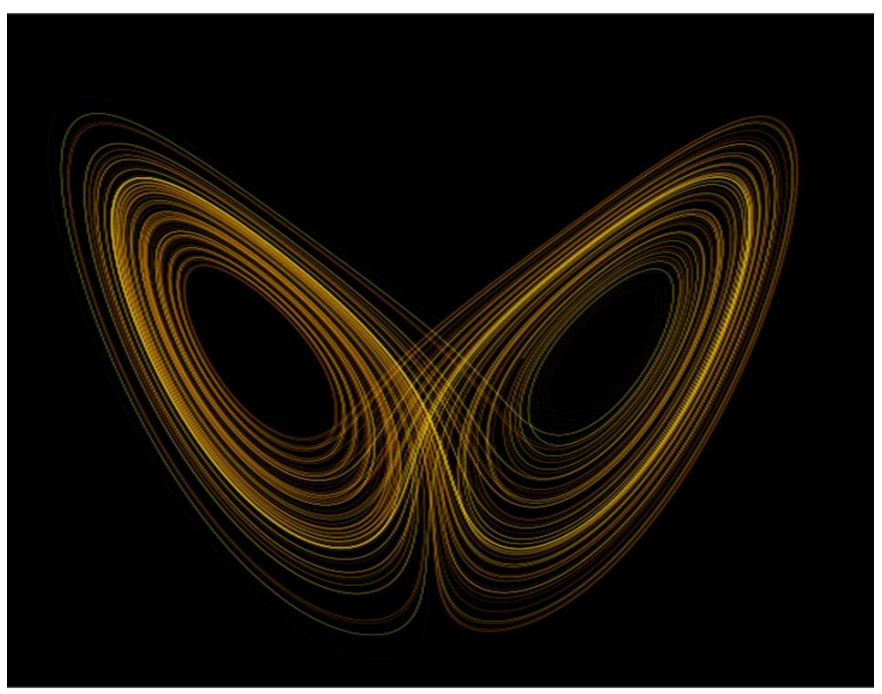

Figure 11 Lorenz strange attractor (Lorenz 1963) 
Self-similarity, as characteristic patterns representing a whole comprising many parts which are a copy of the whole, have been named as 'fractals' in geometric theory by Benoît Mandelbrot (1983), and were initially independent of chaos theory but later used to describe and analyse complexity of irregular shapes in the natural world. Although fractals in their purest sense are comprised of components exactly the same but at different scales, the concept of many parts being connected to make a whole has been applied in relation to complex living systems. The fractal entity, in as much as it is both a whole comprising parts, are alike 'holons' described in chaordic systems theory, where “...structures that are simultaneously autonomous and dependent...evolve to higher orders of 'whole/partness' by virtue of four fundamental capacities possessed by each" (Eijnatten 2004 p.438). Agency, membership, self-transcendence and self-dissolution are the four distinguishing characteristics of holons with emerging levels of complexity being directly attributed to the degree of individual connectedness and resultant emergent collective consciousness. Discontinuous patterns of growth in chaordic systems alternate between periods of stability through gradual change, and periods of chaotic instability when non-linear transformative change takes place as holonic growth increases and multiplies in complexity (Eijnatten 2004). This is also similar to the 'dominant punctuated equilibrium' model of transformational change which is described by Engeström et al (2007b), where organizational change is portrayed as a constant gradual process which reaches a point of eruption resulting in "...intense reorganization ... over a relatively short period of turmoil" (р.320).

The crucial point at which comparing fractals to holons or human social groupings breaks down, is trying to equate the identical nature of fractal parts making up a whole, with the uniqueness of each individual player in a social context. Social processes emerge from truly unpredictable behaviour of disparate heterogeneous groups of interconnected cognitive beings embedded in socio-cultural contexts which are dynamic and evolving. Language as a living system phenomenon emerges from mutual structural coupling between individual elements and the environment, with selfconsciousness arising as a unique reality. Complexity in project practice as for other social entities, results from a mix of patterned and unpatterned, predictable and unpredictable behaviours, with states of being evolving into an ontology of becoming. Parts make up a whole, but they are unique in identity and dynamic in existence.

As noted, central to complexity as a theory or concept is the web of multiple elements interconnected and dynamically related. As has been summarized in Table 1, the autonomous, selforganising, adaptive nature of systems, as living, complex adaptive and chaordic, have been informed by chaos theory, with elements of interconnectedness, emergent consciousness and transformative potentiality being associated with activity systems and social-ecological theoretical frameworks. However, it is the multiplicity and changing nature of heterogeneous elements in social systems, 
which contribute to the chaos arise from complexity, with even the prediction of patterned responses being made difficult due to unique combinations, shifts in power relationships and changes in identity. Projects as social process are complex not only due to the number of component parts, but due to the disparate nature of the parts, together with shifting alliances and emergent forms of cognition. Projects may therefore be better perceived as human activity systems involving cognitive processes where "...a changing mosaic of interconnected activity systems ... are energized by their own inner contradictions" (Engeström 2001 p.140).

Engeström's (2000) activity theory has been informed by Vygotsky's (1978) cultural-historical activity theory which emphasizes the mediating nature of culture in human acts and Leont'ev's (1981) concept of differentiating between individual and collective action. As noted by Engeström (2001), although interrelationships between an individual and their community in these first 'two generations' of activity theory were noted as being complex, with internal contradictions being suggested as a driving force of change by Il'enkov (1977), it was not until globalization contributed to world-wide connectedness (Held, McGrew et al. 1999) that issues of cultural diversity and discursive dialogue acted as the impetus for a 'third generation' of activity theory to emerge. The five principles underpinning Engeström's (2001) conceptualisation of activity systems encompass concepts of networked relations, collectivity and self-reproduction, multi-layered, multi-voiced communities, historicity, contradictions and conflicts as instruments of change, and collective change through expansive transformations.

Engeström's (2006) has proposed that his activity theory of expansive learning involving concepts of 'knotworking', 'swarming' and 'runaway objects' potentially “...lead to future-oriented questions that seek the contours of emerging collaborative organizational forms" (p.1787) to better facilitate organizational transformation through the application of multiple as opposed to singular epistemic tools to accommodate for the new workplace complexity. 'Knotworking' represents the complexity of interactions between actors and activity systems which develop, dissolve then re-form in other temporary configurations, to facilitate collaborative relationships in evolving working environments attempting to grapple with and accommodate for rapid and persistent change (Engeström 2000). 
Table 1 Comparing complex theoretical assumptions

\begin{tabular}{|c|c|c|c|c|c|c|}
\hline Living Systems & $\begin{array}{c}\text { Complex Adaptive } \\
\text { Systems }\end{array}$ & Chaordic Systems & Complexity Theory & Activity Systems & Project Complexity & Social - ecological \\
\hline (Maturana 1999) & (van Eijnatten 2004) & $\begin{array}{l}\text { (Glass 1996; Capra } \\
\text { 1997; Cilliers 2000; } \\
\text { Richardson 2008) }\end{array}$ & (Engeström 2001) & $\begin{array}{l}\text { (Ivory, Alderman et al. } \\
\text { 2006; Cooke-Davies, } \\
\text { Cicmil et al. 2007) }\end{array}$ & $\begin{array}{l}\text { (Law and Urry 2004; } \\
\text { Buchinger 2006; Folke } \\
\text { 2006) }\end{array}$ & \\
\hline Autonomous & $\begin{array}{l}\text { Autonomous } \\
\text { Heterogeneous individual } \\
\text { parts defined by dynamics } \\
\text { of whole }\end{array}$ & Holonic & $\begin{array}{l}\text { Strange attractors } \\
\text { Fractals }\end{array}$ & $\begin{array}{l}\text { Multivoiced } \\
\text { Multiple layers }\end{array}$ & $\begin{array}{l}\text { Autonomous } \\
\text { Socially constructed } \\
\text { Multiple perspectives }\end{array}$ & $\begin{array}{l}\text { Autonomous } \\
\text { Complex whole } \\
\text { irreducible to parts } \\
\text { Self-referential }\end{array}$ \\
\hline $\begin{array}{l}\text { Autopoietic } \\
\text { Network of processes } \\
\text { Self-producing } \\
\text { Recursively- } \\
\text { regenerating }\end{array}$ & $\begin{array}{l}\text { Autopoietic } \\
\text { Self-organising } \\
\text { Control \& order Emergent }\end{array}$ & $\begin{array}{l}\text { Self-organising } \\
\text { Discontinuous growth } \\
\text { Dissipation } \\
\text { Emergent }\end{array}$ & $\begin{array}{l}\text { Self-organising } \\
\text { Dissipative } \\
\text { structures } \\
\text { Fit to environment }\end{array}$ & $\begin{array}{l}\text { Systems take shape \& } \\
\text { transform }\end{array}$ & Self-organising & $\begin{array}{l}\text { Autopoietic } \\
\text { Self-organising } \\
\text { Adaptive, resilient } \\
\text { governance } \\
\text { Self-generating }\end{array}$ \\
\hline $\begin{array}{l}\text { Organizationally closed } \\
\text { unity with boundaries }\end{array}$ & Non-linear & Non-linear & $\begin{array}{l}\text { Non-linear } \\
\text { Boundary objects } \\
\text { Incompressible }\end{array}$ & $\begin{array}{l}\text { Collective object- } \\
\text { oriented }\end{array}$ & $\begin{array}{l}\text { Non-linear } \\
\text { Boundary objects }\end{array}$ & $\begin{array}{l}\text { Multi-level governance } \\
\text { systems } \\
\text { Non-linear } \\
\text { relationships }\end{array}$ \\
\hline $\begin{array}{l}\text { Structurally opened } \\
\text { Reciprocally } \\
\text { structurally coupled in } \\
\text { environment }\end{array}$ & $\begin{array}{l}\text { Entities are open part of } \\
\text { other networks } \\
\text { Co-emergence } \\
\text { Interdependence } \\
\text { Interconnected }\end{array}$ & Connectivity & $\begin{array}{l}\text { Interconnections in } \\
\text { phase space } \\
\text { Pluralistic }\end{array}$ & $\begin{array}{l}\text { Open systems } \\
\text { Contradictions between } \\
\text { systems generate change } \\
\text { Network relations }\end{array}$ & $\begin{array}{l}\text { Interlinked social } \\
\text { worlds } \\
\text { Interdependent } \\
\text { elements }\end{array}$ & $\begin{array}{l}\text { Collective action } \\
\text { Social networks } \\
\text { Global networks } \\
\text { Fuzzy boundaries } \\
\text { Unstable }\end{array}$ \\
\hline $\begin{array}{l}\text { Language as cognition } \\
\text { arises in consensual } \\
\text { domains } \\
\text { Mutual reciprocity }\end{array}$ & $\begin{array}{l}\text { Phase transitions } \\
\text { Flows } \\
\text { Emerging forms } \\
\text { Ability to learn \& adapt } \\
\text { Generate new responses }\end{array}$ & $\begin{array}{l}\text { Consciousness } \\
\text { Indeterminate }\end{array}$ & $\begin{array}{l}\text { Behaviour } \\
\text { deterministic \& } \\
\text { patterned } \\
\text { Evolving }\end{array}$ & $\begin{array}{l}\text { Expansive qualitative } \\
\text { transformations } \\
\text { Deliberate collective } \\
\text { change through } \\
\text { transformations }\end{array}$ & $\begin{array}{l}\text { Interplay of discourse } \\
\text { Social interaction } \\
\text { Social processes for } \\
\text { sense making }\end{array}$ & $\begin{array}{l}\text { Knowledge systems } \\
\text { respond to \& shape } \\
\text { ecosystems } \\
\text { Transform } \\
\text { communication } \\
\text { Emergent } \\
\text { consciousness } \\
\text { Mutual reciprocity }\end{array}$ \\
\hline Dynamic & Dynamic & Dynamic & Edge of chaos & Unstable activity & Unpredictable & $\begin{array}{l}\text { Dynamic } \\
\text { Unpredictable }\end{array}$ \\
\hline
\end{tabular}


Project praxis in this sense may be seen as a continuum of human activity systems involving shortlived, multiple objects of orientedness, where collective activity emerges from a balance of continuity and discontinuity involving enacted cognition through 'negotiated knotworking' as a social process. Project actuality also reveals adaptive flexibility to accommodate for 'run away objects' (Engeström 2006) which as a concept informed by the works of Anthony Giddens (1990; 2000), are by-products resulting from a rapidly changing complex world in which uncontrollable objects arise, akin to environmental perturbations which may serve as threats or opportunities to system resilience. Cycles of expansive learning therefore reflect the transformational activity arising from contradictions, conflicts or disparate understandings in and through networking activities. This is supported by assuming there is mutual engagement for the production of "...culturally new patterns of activity...[and]...new forms of work activity" (Engeström 2001 p.139).

Projects as complex human activity systems refocus the theoretical lens on the individual as the primary element embedded in context, who through being structurally coupled with other individuals together with the environment, co-emerge and change through dynamic knotted interactions. Enactivism as a theory and methodology for learning complements Engeström's activity conceptual framework, emphasizing learning through language as cognition, with self-organisation modifying behavior reciprocally creating change in its world (Reid 1996). Enactivist theory views “...learning and knowing as complex, emergent processes by which dynamic agents maintain fitness with one another and within dynamic contexts ...[and] ...the knowing agency emerges from, and is nested in other complex systems" (Begg 2002 p.4). Projects as human activity systems where individuals are intertwined, are a microcosm of complex social systems, whose mutual survival is codependent on their interactions (Luhmann 1995; Cicmil and Hodgson 2006). Behaviour which contributes to project team survival relies on collective activity which is persistent, creative and sensitive to environmental triggers. Project practice if then conceptualized as an emergent human activity, may be seen to involve co-ordinated collaborative 'swarming' movements to combine outward 'action and pursuit' with inward regathering and reflective practice (Engeström 2006).

The actuality of projects as complex human activity systems, where expansive learning as emergent cognition underpins organizational transformation, involves language as dialogue in a consensual domain of interactions. The project entity is able to balance continuity with discontinuity, as an ontology of being and becoming, necessitating recursive descriptions through interactions and interconnections for emergent self-consciousness (Maturana 1999). Project praxis is situated in a web of organizations horizontally networked with other organizations on an international scale, where strategic alliances are alike symbiotic relationships (Kelly 1998). Project actuality has the potential to create connectivity on a global scale through having players working on more than one project at a time or being a member of more than one project at a time; projects with work sub 
contracted and subcontracted again. The realities of the social processes involved in such interconnected human activity systems will inevitably entail socio-cultural differences originating from disparate historical roots and discursive language underpinnings. Project management in this new era creates challenges where the solution is unlikely to be found in traditional control models of instrumentality. The complexes that exist which are seeing "...an unfolding interplay between language, action and structure has produced a model of managing projects that bears no resemblance to the sanitized world of the project management literature" (Green 2006 p.249)

Management of projects will need to move beyond the controlling paradigm to new and emergent forms of leadership where envisioning and anticipating the future for organizational adaptation will need to be tempered with the reality that complexity assumes it is impossible to model or predict a world whose ontology is no longer singular (Law and Urry 2004). Both context-determined and global factors are contributing to the increasingly complex project environment and although these elements have been instrumental in driving an increase in project-based management (Hobbs, Aubry et al. 2008), the traditional project management models incorrectly assume environmental sensemaking can be based on positivist control (Whitty 2005). An enactivist view more ably complements expansive learning through visualising projects as human activity systems to support emergent forms of project management. "The social systems of project management comprise a myriad of microworlds populated by actors who continuously engage in a process of sense-making" (Green 2006 p.235). Facilitating individual interpretation in complex human activity systems when working towards collective consciousness may require creative 'knotworking' and guided swarming, particularly when social realities are discursive. The competing narratives and discursive practices that exist as power plays in project practice (Boddy and Paton 2004; Green 2006) need to be realized. Project praxis as organizational functionality "...may emerge through conversation, but they do not emerge for the sake of conversation" (Engeström 1999a p.170). Acknowledging the dynamics of learning through dialogue embedded in context, has greater potential to optimize benefits from heterogeneous networks involved in projects practice, with emergent forms of leadership supporting autonomous human activity through relinquishing instruments of control.

\subsection{Chapter Summary}

This chapter has presented cognition as the process of knowing as being inextricably linked with consciousness which in turn assumes a self-awareness and ability to 'know what we know' (Freire 1970). The communication of what we know, how we know, how we interact with others, how we 'learn' and how knowledge is created and transmitted, necessitates linguistic communication that has developed to the point of 'languaging' (Bloom, Engelhart et al. 1956; Skinner 1974; Maturana 
1995); where languaging is " ... as a manner of flowing in recursive consensual coordinations of behavior ... is a manner of living in coordinations of doings" (Maturana 1995 p.1) and 'words' are constituted through reflection and action for praxis (Piaget 1937/1954). It is then discourse as a cognitive human function which can facilitate an emergent ontological shift from 'being' in a world to 'becoming' through action and doing (Berger and Luckman 1967; Vygotsky 1978). Cognition as key to 'becoming' is considered "... not an individual process but instead involves the individual, the social collectivity to which he/she belongs and the socially legitimated objects of inquiry... a socially conditioned response to a pre-established ordering of things" (Chia 1995 p.582).

It is this social nature of learning through interconnections which is of relevance to project research and practice. Active sharing of experience and redefining of realities that align pragmatic forms of inquiry with holistic approaches to cognition move beyond reductionist thought and passive concepts of learning. The discursive nature of communicative dialogue in culturally complex project environments is considered to reflect the essence of human cognition as collective consciousness, where the "... locus of knowledge is no longer taken to be the individual mind but rather to inhere in patterns of social relatedness" (Gergen 1985 p.471).

The complex nature of today's working environment, where rapid decision making is necessary in an era of ambiguity and uncertainty, benefits from thinking about project practice within an enactivist framework. The embodied nature of cognition where learning through inquiry is situated in a domain of constitutive ontologies, assumes collective consciousness is an outcome of individuals intersubjectively connected with each other. Such a contextual reality has the potential to facilitate organizational transformation from a state of being in the world to a becoming entity. Projects may be seen to provide the structural openness necessary for organizationally closed systems to adapt to perturbations in the environment, with sensemaking activities being supported by interpretive processes involving 'becoming aware'.

Project complexity has traditionally been associated with technical and structural elements of project management practice, with systems of operational planning and control dominating (Atkinson, Crawford et al. 2006). Yet given the rapidity of change in a globalised world, where technological developments in communication systems and ease of international travel have contributed to increasingly permeable nation-state borders, a new social plurality is entering national landscapes. This creates new 'multi-dimensional' dilemmas in the workplace, which are both socio-culturally and cognitive-emotionally derived (Höijer, Lidskog et al. 2006). Perceiving social systems as complex adaptive systems, unstable, emergent and self-organising, reflect the dynamism of today's organisational life. Webs of human interrelationships which are inseparable, discontinuous and nonlinear are inherent to complex social systems comprising heterogeneous socio-cultural mixes. This 
human plurality demands exploration into relevant sensemaking activities through acknowledging language as cognition in emergent living systems.

Enactivism supports the concept of emergent entities, with cognition being pivotal to structural change for adaptive learning and organizational resilience (Reid 1996; Maturana 1999; Folke 2006). Project management practice embedded in context, is enacted through recursive interactions between individuals coupled with each other and the environment. As suggested, "knowing is being is doing' (Reid 1996), reality is relational, and coherence arises in and through language to facilitate a collective consciousness (Maturana 1988). Social complexity occurs from cultural and language disparities, with divergent ways of knowing underpinning a diversity of assumptions as being informed by individual experience and environmental influences. The resultant discursive dialogue and competing narratives (Boddy and Paton 2004; Green 2006) reflect a plurality in understanding despite apparent superficial social homogeneity in dominant language used in communication.

As J Rodney Turner (1999) has noted, "Project management really is a social science... which will be applied differently by different cultures" (p. 493). When uncertainty and/or ambiguity exist within an organizational setting and project goal clarity is 'fuzzy', the 'one size fits all' approach to project management is bound to falter (Söderlund 2004a; Pollack 2007). The real challenge therefore for project managers is to know how to 'manage' knowledge flows and action when the organization is culturally complex and an essence of mutual understanding is elusive. Dialogue may facilitate movement toward mutual action, but it is questionable whether 'shared understandings', particularly with culturally diverse actors, is possible (Derrida 1992). Knowledge creation has been presented as being a result of co-ordinations of behaviour in consensual domains (Maturana 1995), interrelationships in co-operative domains (Whitaker 1995) or as a result of assimilated meanings (Freeman 1999). Yet given the subjective nature and cultural specificity of individual experience, seeking consensus in meaning per se is a questionable construct. Acknowledging the uniqueness of individual understanding and the existence of different social agendas and power relations that exist “...rooted deep in the social nexus..." (Foucault 1982 p.208) in a project environment, may be pivotal to 'consensus in action' as opposed to 'consensus in meaning' for improved project outcomes. Project complexity arising from human plurality warrants exploration for its implications to projects as social process.

Gaps in the literature indicate a need to explore: relationships between projects and their environment (Söderlund 2004a); projects as complex social arrangements (Cooke-Davies, Cicmil et al. 2007); project contexts as complex with multiple understandings (Ivory, Alderman et al. 2006); new project trajectories as social phenomena (Cicmil and Hodgson 2006); and projects as involving complex social processes (Winter, Smith et al. 2006). This shift in focus from more structured, instrumental approaches to project management parallel discussions for new approaches to social 
analyses to move away from modernist frameworks focusing on structural control systems of inquiry (Law and Urry 2004). Models of management utilizing socio-ecological frameworks (Riegler 2002; Buchinger 2006; Folke 2006; Limone and Bastias 2006; Parboteeah and Jackson 2007), emphasizing the inseparable nature of context and action in an increasingly diverse and pluralistic world (Pettigrew, Woodman et al. 2001b; Orlikowski 2002; Boddy and Paton 2004; Höijer, Lidskog et al. 2006), are being called for. The research in this dissertation thus aims to explore project complexity as socio-culturally derived, to contribute towards widening the theoretical base for improved project outcomes. 


\section{Chapter 3 Methodology}

\subsection{Introduction}

The directions for future project management research for practice have been identified as encompassing three broad practice areas: theory about practice, theory for practice and theory in practice (Winter, Smith et al. 2006) Table 2. Different theoretical bases for each of these areas have been influenced by whether knowledge of the projects as process is 'pre-existing' or 'given' or whether the project research process emerges as a 'becoming' entity. The Winter, Smith et al's (2006) 'future' research model suggests an evolving position 'from' what exists in current research 'towards' an enhanced position, as is reflected in postmodern thinking where an ontology of “...action, movement, process and emergence" (Chia 1995 p. 597) dominates. Projects as emerging processes working in and through complex non-static evolving environments are informed by Winter, Smith et al's (2006) principal findings which indicate there is "... the need for new thinking in the areas of project complexity, social process, value creation project conceptualisation and practitioner development" (p. 641). The research project described in this dissertation fits into the 'theory for practice' camp, with an aim to shift thinking 'from' projects as instrumental processes 'towards' considering them as social processes involving people interacting with others (Direction 2). Such potential complexity demands new ontological and epistemological approaches to challenge the preexisting rationalist models of project management (Winter, Smith et al. 2006) to bring forth new ideas and alternative directions (Dodouras and James 2007) .

Conventional project management research and practice has been heavily influenced by positivism, rationalism and modernist thought within a 'hard' systems paradigm (Winter and Checkland 2003; Söderlund 2004a; Cicmil, Williams et al. 2006; Crawford, Pollack et al. 2006; Lineham and Kavanagh 2006; Winter, Smith et al. 2006; Pollack 2007). While this does not negate the value added to existing project management knowledge, the viewing of projects as standardised, objective goal directed endeavours fails to adequately address the realities of social complexities, ambiguous working environments and social interactions which are critical to project success. Calls to broaden the base of project management research have been made, and although it is noted that one encompassing theory is unrealistic, a paradigm that accommodates the actualities of 'messy' projects and uncertain 'fuzzy' problems is needed (Williams 1999; Winter and Checkland 2003; Söderlund 2004a; Cicmil 2006; Cicmil, Williams et al. 2006; Winter 2006). 
Rethinking Project Management (EPSRC Network 2004-2006)

Directions for Future Research

IMPORTANT NOTE: the word 'Towards' means to enhance the 'from' position rather than to discard it.

\section{Theory ABOUT Practice}

The Lifecycle Model of Projects and PM

From: the simple lifecycle-based models of projects, as the dominant model of projects and project management.

And from: the (often unexamined) assumption that the lifecycle model is (assumed to be) the actual 'terrain' (i.e. the actual reality 'out there' in the world).

\section{Direction 1}

Theories of the Complexity of Projects and PM

Towards: the development of new models and theories which recognise and illuminate the complexity of projects and project management, at all levels.

And towards: new models \& theories which are explicitly presented as only partial theories of the complex 'terrain'.

\section{Implication}

The need for multiple images to inform and guide action at all levels in the management of projects, rather than just the classical lifecycle model of project management, as the main guide to action, (with all its codified knowledge and techniques). Note: theories ABOUT practice can also be used as theories FOR practice.

\section{Theory FOR Practice}

\section{Direction 2}

Projects as Instrumental Processes

\section{Projects as Social Processes}

From: the instrumental lifecycle image of projects as a linear sequence of tasks to be performed on an objective entity 'out there', using codified knowledge, procedures and techniques, and based on an image of projects as temporary apolitical production processes.
Towards: concepts and images which focus on social interaction among people, illuminating: the flux of events and human action, and the framing of projects (and the profession) within an array of social agenda, practices, stakeholder relations, politics and power.

\section{Direction 3}

\begin{tabular}{|c|c|}
\hline Product Creation as the Prime Focus & $\Rightarrow$ Value Creation as the Prime Focus \\
\hline $\begin{array}{l}\text { From: concepts and methodologies which focus on: } \\
\text { product creation - the temporary production, development, } \\
\text { or improvement of a physical product, system or facility } \\
\text { etc - and monitored and controlled against specification } \\
\text { (quality), cost and time. }\end{array}$ & $\begin{array}{l}\text { Towards: concepts and frameworks which focus on: } \\
\text { value creation as the prime focus of projects, programmes } \\
\text { and portfolios. Note however: 'value' and 'benefit' as } \\
\text { having multiple meanings linked to different purposes: } \\
\text { organisational and individual. }\end{array}$ \\
\hline
\end{tabular}

\section{Direction 4}

\section{Narrow Conceptualisation of Projects $\Rightarrow$ Broader Conceptualisation of Projects}

From: concepts and methodologies which are based on: the narrow conceptualisation that projects start from a well-defined objective 'given' at the start, and are named and framed around single disciplines, eg. IT projects, construction projects, HR projects etc.
Towards: concepts and approaches which facilitate: broader and ongoing conceptualisation of projects as being multidisciplinary, having multiple purposes, not always pre-defined, but permeable, contestable and open to renegotiation throughout.

\section{Theory IN Practice}

\section{Direction 5}

\section{Practitioners as Trained Technicians}

From: training and development which produces: practitioners who can follow detailed procedures and techniques, prescribed by project management methods and tools, which embody some or all of the ideas and assumptions of the 'from' parts of 1 to 4 .

\section{Practitioners as Reflective Practitioners}

Towards: learning and development which facilitates: the development of reflective practitioners who can learn, operate and adapt effectively in complex project environments, through experience, intuition and the pragmatic application of theory in practice. 
The research project described by this dissertation is guided by a philosophical conceptual framework (Figure 4) which incorporates epistemological and ontological assumptions drawing upon enactivist theory and postmodern thinking within a soft systems paradigm. Viewing projects as social process introduces the notion of emergent cognition being enacted through a web of interconnected human elements. Knowledge emerges through language from individuals being embedded in a socio-cultural environmental context with thinking being underpinned by a methodology that is informed by enactivist descriptions of the nature of reality and the nature of cognition. As suggested by Richardson (2008) a researcher's choice of a particular methodology is "...underpinned by particular views of how the world we observe is constructed and how it should respond to our actions upon it" (p.12).

A conceptual framework has therefore been designed to explore what was a messy problematic situation in a state of 'flux' and continual movement. A shift from traditional positivist methodological approaches in project management research offers insight into the issues of communication and knowledge creation in culturally disparate social groups involved in project practice. A pragmatic epistemology and a becoming ontology form the philosophical conceptual base of the scientific enquiry. An action research strategy supporting a pragmatic epistemology, presupposes that in the social world the interaction of two types of experience - "knowledge of acquaintance' and 'knowledge about' - are connected through action and reflection for theory building and knowledge generation (Calori 2002). After considering the research problem and the related issues as detailed in Section 3.2, Soft Systems Methodology (SSM) was selected as the research methodology since its design bridges the divide between normative and descriptive research. This was considered better able to address the socio-cultural aspects inherent to project practice through emphasising 'learning' rather than 'solving' (Winter and Checkland 2003).

The sections in this methodology chapter provide an overview of the research problem, then a description of the thinking behind the choice of paradigm and research approach. This is followed by an overview of the conceptual framework designed to explore the research problem. The choice of research strategy is discussed in the context of a number of other research approach options, prior to expanding upon the rationale for selecting SSM and the method implemented for the research process. Issues of rigour in relation to reliability and validity of the research process are then considered, with a review of relevant ethical issues concluding the chapter. 


\subsection{The Problematical Situation}

The research project described in this dissertation was instigated as a result of difficulties encountered in the start-up and execution stages of a Quality Assurance (QA) project in a higher educational institution in the United Arab Emirates (UAE). The problematical situation involved a situation that needed to have something done about it - i.e. not a problem to solve, rather a situation that could benefit from improvement (Checkland and Poulter 2006). The QA process was part of a larger institutional accreditation initiative in a system comprising 12 colleges, two in each Emirate, plus centralised academic and administrative service departments. The research project described by this dissertation is limited to one of the 12 colleges, although as a member of the overall institutional QA committee, I as researcher had an appreciation of system wide issues being raised across colleges. In the college which gave approval for this research project as described, a number of factors which are not uncommon to new initiative projects, such as poor stakeholder buyin, obstruction of process, inertia to change, inconsistent resource allocation and miscommunication in and between colleges, were problems which seemed to indicate more complex underlying problems. The institute is staffed by a globally sourced expatriate community who are on short term employment contracts working within the institute's Arabic system of governance. Project management and attempts to empower individuals and teams with QA processes as improvement tools did not progress in the direction or at the rate needed to achieve desired and expected outcomes. Despite these concerns being raised with senior management, and strategies to improve the situation discussed and trialled, momentum at the project level never seemed to increase.

While some of these difficulties are not unfamiliar in other project contexts, from my perspective as project manager three questions seemed to succinctly articulate the problem situation:

1. What factors influence organizational learning in a pluralistic socio-cultural context which is diverse and complex, and how can they be strategically managed for advantage?

2. How can organizations with transient human capital utilize quality improvement programs to capture, create and exploit knowledge to ensure sustainable capability?

3. How can project management accommodate embedded socio-cultural factors acting as barriers to organizational change?

This research project originally aimed to gain an understanding of factors which influence organizational change and how these factors related to the implementation of a quality improvement program. The pluralistic socio-cultural diversity in expatriate employees and the dichotomy between the Arabic governance system and expatriate organisational management contributed to a number of communication issues, but the problem seemed to be more complex and 
messy than simple misunderstandings. Therefore a number of 'initial' aims in the research project (see 1.5 Introduction section) were raised in the exploratory stage when inquiry commenced, with these evolving and being refined, as follows:

\section{Aim 1:}

To explore the significance of socio-cultural context, socio-technical infrastructure and human capital elements relevant to managing projects for effective organizational change

\section{Aim 2:}

To develop a project management technique or strategy which can:

a) accommodate culturally disparate human capital to,

b) facilitate communicative action and knowledge transfer to,

c) promote sense-making for purposeful activity and improved organizational capability

\subsection{The choice of paradigm}

A 'paradigm' represents a way of thinking and viewing reality, and is commonly used to frame or organize theory and research (Neuman 2006). It is a term that was introduced by Kuhn (1962) to describe a set of assumptions or techniques that a particular scientific community may adopt to explain systems of enquiry. As a construct a 'paradigm' "...specifies a general set of philosophical assumptions covering...ontology (what is assumed to exist), epistemology (the nature of valid knowledge), ethics or axiology (what is valued or considered right) and methodology" (Mingers 2001 p.242). As represented in Figure 12, a research activity draws upon theoretical traditions in selecting a suitable methodology to frame the enquiry process. The problematic situation described in this dissertation acted as the impetus for the research, beginning with the experiencing of a situation, reflecting upon purposeful actions that were or were not working in project implementation, followed by defining of an issue or area of study worthy of research. Theoretical traditions of project practice in relation to the situation were explored, with subsequent assumptions about a relevant conceptual framework to underpin the realities of project praxis preceding selection of inquiry, data collection and interpretive methodologies.

Whilst dominant assumptions framing the research described in this dissertation are drawn from what is perceived as the 'soft' paradigm, the integrated conceptual framework designed also draws upon enactivism as a cognitive theory (which may be viewed as a paradigm itself) and postmodern thinking. These combine to support the notion of a becoming ontology and a pragmatic epistemology to guide the problem structuring approach utilising Soft Systems Methodology. Such a pluralistic approach is seen to reflect the complexity of the situation under investigation and the pluralism 
which dominates today's 'multi-dimensional' world, and has been designed to enrich the project management theoretical and practice base. This 'multiparadigm' approach has the potential to establish a "...more rich, holistic and contextualized purview" (Lewis and Grimes 1999), and supports the call to develop 'alternative theoretical frameworks' from which new combinations of methodologies may be trialled (Mingers 2001).

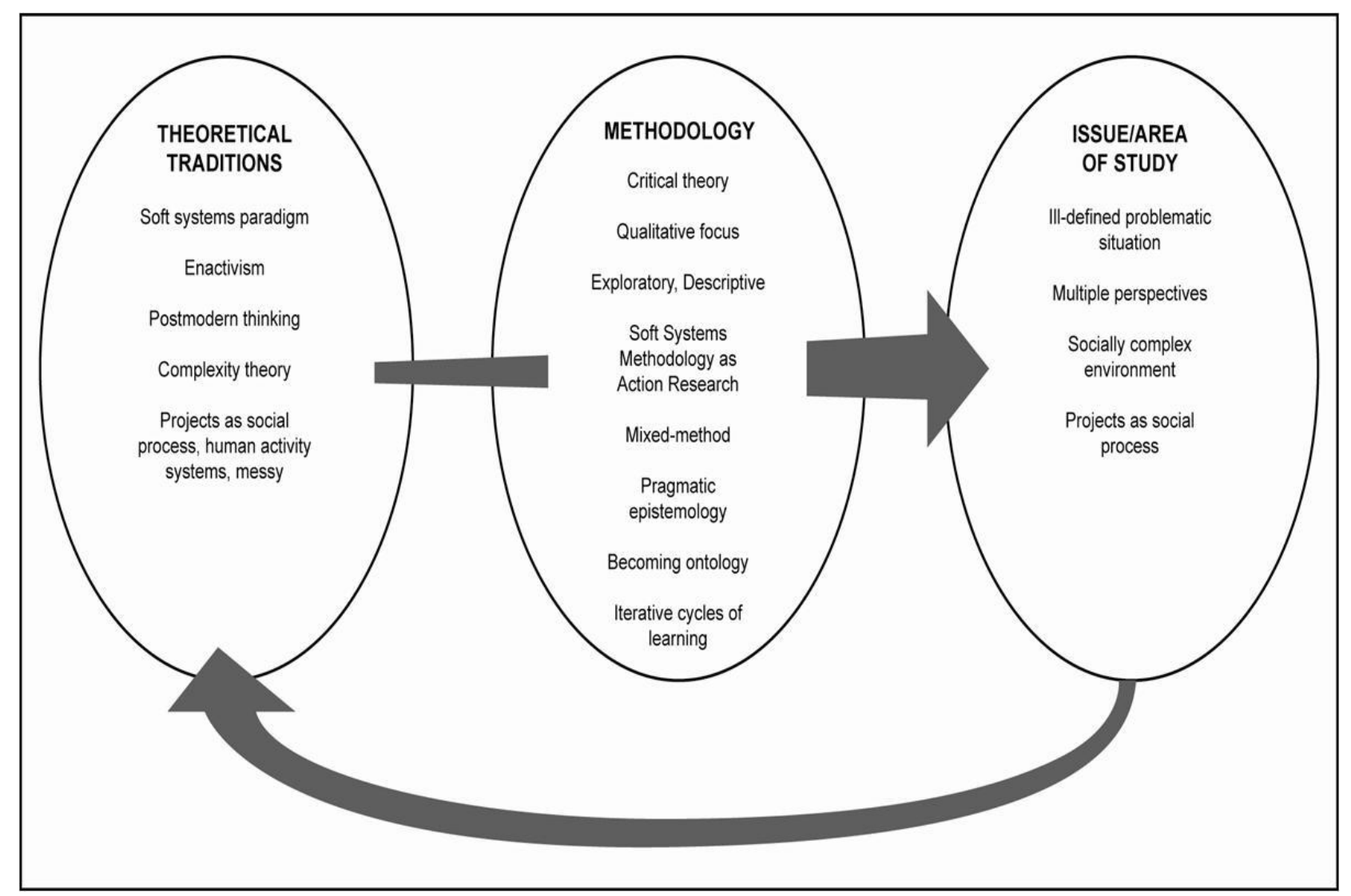

Figure 12 The research activity as a knowledge creation process (adapted from Cicmil, 2006 p.29)

\subsubsection{Hard Systems or Soft Systems?}

It has been suggested that project management per se embodies a 'hard systems' paradigm or world view, where positivist, reductionist, rational and normative thinking dominates (Cooke-Davies, Cicmil et al. 2007; Pollack 2007). With paradigms not only framing enquiry, but also influencing enquiry through portraying a particular image and determining ways of thinking (Winter and Checkland 2003; Reisman and Oral 2005; Pollack 2007), it is not surprising that given the historical traditions of project management lying in the construction, information and service sectors (Crawford, Pollack et al. 2006), that normative, prescriptive, rational, positivist research dominates the field of project management enquiry (Cooke-Davies, Cicmil et al. 2007; Pundir, Ganapathy et al. 2007). 
Project management practice and research have been noted as having two main sources of intellectual tradition - one lying in the engineering or mathematical sciences with emphases on method and planning techniques to solve reasonably well-defined problems, and another associated with the social sciences where the focus is on organisational behaviour in project organisations (Söderlund 2004a). What appears to be evidence of an increase in project complexity, both in practice and in environment, has instigated a realisation about the inadequacy of traditional rational approaches to project management theory, with claims that the relevance of the current conceptual base is lacking (Winter, Smith et al. 2006).

Although new project research directions have been sought to address this apparent emerging 'complexity' in projects, no singular definition of 'project complexity' seems to draw agreement (Williams 1999). The literature however, does seem to indicate a consistency in focusing on systems thinking and complexity theory in attempts to conceptualise and address the problem situations appearing in project practice (Williams 1999; Williams 2002; Cicmil 2006; Cicmil, Williams et al. 2006; Cooke-Davies, Cicmil et al. 2007; Pundir, Ganapathy et al. 2007; Whitty and Maylor 2007). Central to these explorations is the recognition of the emergent cognitive nature of project praxis that sits outside models of instrumentality and control which typify the Cartesian mechanistic paradigm. The search to find conceptual models that support a becoming ontology where knowledge arises through individuals connected with one another embedded in context, suggests a reality where projects as a social system rely on circular organized communication for survival (Buchinger 2006).

The research presented in this dissertation has noted the social nature of complexity in the actuality of projects and has turned to sociological theories that move beyond positivist assumptions of organizational behavior, to consider research with philosophical and methodological assumptions that better address the realities of emergent cognition for project practice. It is felt that systems thinking, where the world is seen as an integrated whole whose properties cannot be seen in isolation from the whole (Capra 1983) serves as a useful starting point for the conceptual approach. For purposes of inquiry into project complexity the organic or 'soft' concept of a system where the focus is on the processes rather than the project as an objectifiable entity, or on 'Problem Structuring' rather than 'Problem Solving' (Pollack 2007), is considered more relevant given the assumed 'living' and cognitive nature of the project system.

The origins of systems thinking lie in early $20^{\text {th }}$ century organismic biology, where a system came “...to mean an integrated whole whose essential properties arise from the relationships between its parts, and 'systems thinking' the understanding of a phenomenon within the context of a larger whole" (Capra 1997 p.27). Systems thinking was applied to quantum physics, Gestalt psychology, and ecology of living systems, but until the time of von Bertalanaffy's (1968) General Systems theory, the focus was on 'connectedness, relationships and context' rather than processes involving 
feedback loops and dynamic connections (Capra 1997). Systems were traditionally defined and recognised by an observer as a reified entity, with this thinking still dominating positivist science today where analytical approaches to studying a system involve reducing the whole to disconnected parts. Reality and epistemology separate the observer from the process of knowing, with such methodology being typical of 'Hard Systems Thinking' (HST) which has guided the solving of welldefined problems in situations which are frequently assumed as given (Checkland and Scholes 1990).

The notion of living systems as 'open systems' was introduced by von Bertalanaffy (1968) through applying biological thinking to the physical sciences in an attempt to expand the theoretical base of scientific thought. His concept of system entropy as being reducible through processes and interconnections of a system with an external context, introduced the idea of self-regulation which has become associated with patterns of interconnection and concepts of circular causality typical of 'new systems' thinking. The focus in this 'new' paradigm is on the process of networks and the inseparable nature of reality where epistemology is indivisible from the object of enquiry, and knowledge is considered approximate rather than certain (Capra 1997). Entropy, as increasing disorder and complexity in living systems is 'stabilised' by autopoietic processes to balance change, the self-organising nature of the system being described in the theory of 'dissipative structures' by Prigogine (1967). These structures are comparable to the concept of a 'being' ontology co-existing with a 'becoming' ontology, where emergent change facilitated by structural openness of an organisational system is balanced with stability of the organisationally closed system to promote resilience and survival.

Adaptation of a system applying HST relies on the ability of a system to sense an environmental change which is conducive to the overall goals of the system, which in turn necessitates whole system changes for survival (Reisman and Oral 2005). Problem solving, analytical deduction and logico-rational approaches all fit within this paradigm and are reflected in traditional models of project management, where the project is a reified entity and a 'being' ontology is dominated by an environment of control and certainty (Lineham and Kavanagh 2006; Pollack 2007).

'New' systems thinking emphasising process moves away from traditional mechanistic approaches dominating Cartesian thought. Ecological and biological thinking has extended the concept of living systems as self-regulating 'open systems' comprising a web of interconnected and interdependent elements. Paradigmatic assumptions in this other 'half' of systems thinking has become known as 'Soft Systems Thinking' (SST) with a focus on communication and control processes which assure adaptive responses of whole entities, with or without layers or sub-sections, but which demonstrate emergent properties as a whole (Checkland and Poulter 2006). According to SST, it is the presence of the human element in a system which makes the system open and dynamic (Reisman and Oral 2005). In turn, it is cognition which determines the living status of a system, where cognition as a process of 
knowing involves perception, emotion and action together with language, conceptual thinking and human consciousness (Capra 1997). Projects when viewed as complex adaptive systems, involve human activity that responds to and creates change in context through recursive processes. Adaptations through processes of learning are enacted as a result of interconnections between individual system components and the environment for self-organization. The autonomous nature of systems in SST (as opposed to control in HST) are informed by the biological concept of autopoiesis, where dynamics and autonomy exist as the two key features of living systems (Maturana and Varela 1980; Buchinger 2006) and processes of communication act as the social process ensuring system survival (Luhmann 1995).

Both HST and SST investigate dynamics of problematic situations, and in some cases both forms of thinking are combined in methodologies as integrated approaches in addressing a problem (Reisman and Oral 2005). HST and SST as defined in paradigmatic terms, are also typically aligned with philosophies that share similar assumptions, as well as being identified with particular research methodologies influencing both perceptions and actions (Pollack 2007). The primary distinguishing feature between the two paradigms as being significant to project management is that HST may be seen to focus on project design and structure to improve functionality of systems, whereas SST supports the view of projects as human activity systems involving social processes with realities being determined by individual perceptions and ascription of meaning.

The research project described in this dissertation involves applied social research, drawing upon theoretical assumptions that help explain and organise knowledge about the social world of project practice. The philosophical conceptual framework underpinning the dissertation views projects as social process (Pettigrew 1987; Green 2006; Winter, Smith et al. 2006), as human activity systems (Checkland and Scholes 1990; Engeström 1999a), as socially complex (Cooke-Davies, Cicmil et al. 2007) and as messy, cyclic, in a state of flux (Winter and Checkland 2003). The selection of a soft systems approach in this research dissertation asserts that difficulties faced in project implementation exist in a complex social world comprising a mosaic of interconnected realities inseparable from the environmental context. The social organisation of 'projects as systems', creates an inherent complexity which results in the 'whole' project entity exhibiting properties which transcend individual components. Cognition emerges from this project system of multiple realities, with interdependent relationships being integral for processes of adaptation, creation and transformational change.

SSM as a process of social inquiry is drawn from SST, representing a shift in thinking about the world as comprising interacting systems which can be 'engineered to work better'(HST), to seeing the 'process of inquiry' or the process of dealing with the world, being the system (Checkland and Scholes 1990). Although this thinking underpins a structured approach to making improvements in 
the world, it negates the assumed embedded nature of humans as cognitive beings in the world. Checkland (1995) refers to human activity systems as being devices to explore the world rather than as existing in a world, with such constructive assumptions portraying the 'system' as being perceived - thus separating the knower from the known. For this primary reason, enactivism as a cognitive theory which extends upon radical constructivism, was seen as a more relevant theory to drawn upon when defining the conceptual framework designed for this research. The complex nature of all living systems where the nature of reality and knowledge assumes the "...elimination of the dichotomy between subject and object" (Proulx 2008 p.24) facilitates the bringing forth of a world to accommodate for adaptation and resilience. Figure 13 is drawn from Checkland and Scholes (1990 p.A11) to portray how viewing the world from an enactivist perspective supports and extends upon SST in relation the nature of reality. 

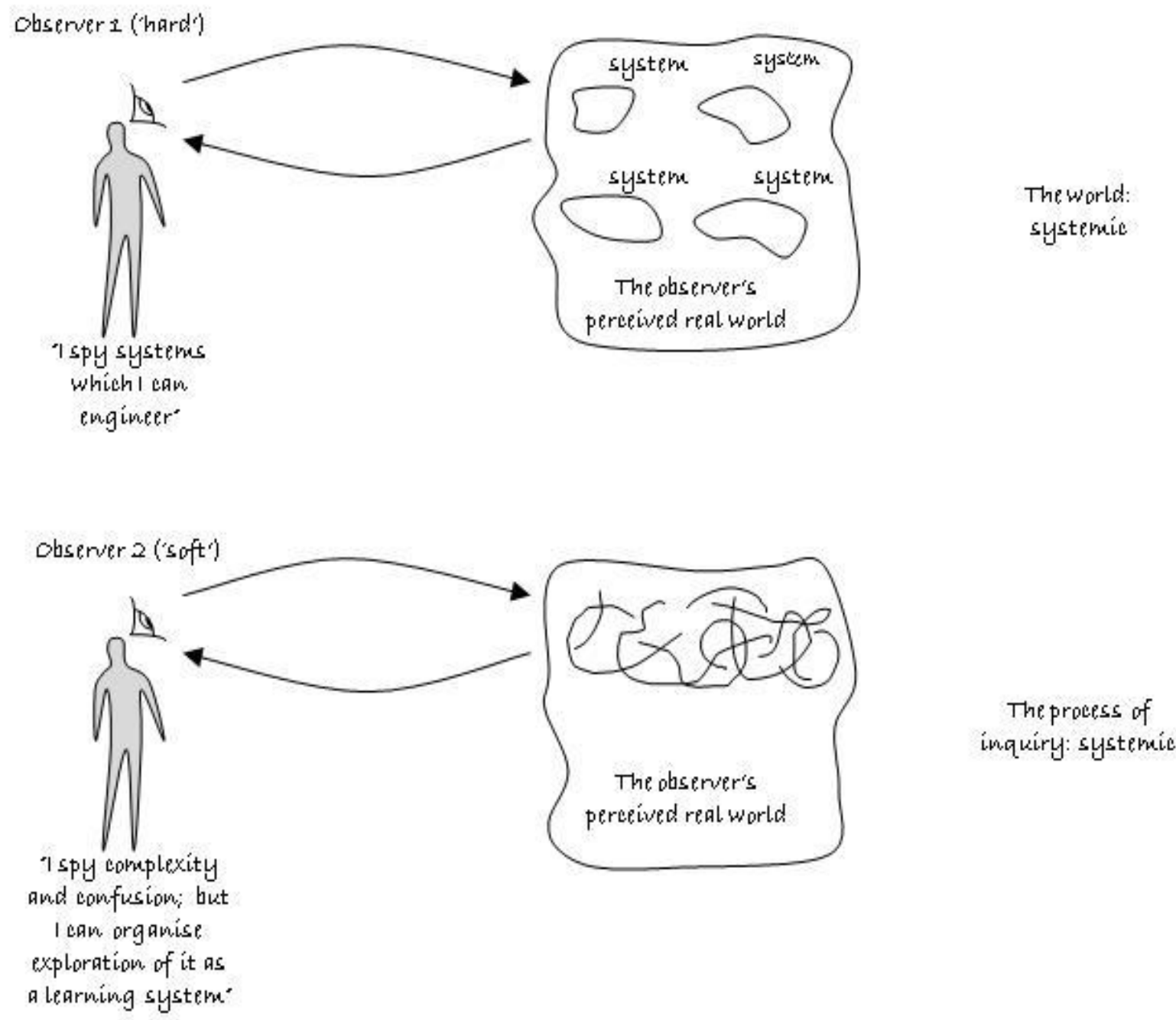

$$
\begin{aligned}
& \text { observer } 3 \text { ('Enactivist') } \\
& \text { 'Ispy a real-world of } \\
& \text { imherent complexity } \\
& \text { from being embedded in } \\
& \text { context inextricably } \\
& \text { eonnected with others to } \\
& \text { bring forth a world } \\
& \text { where egnition is } \\
& \text { emergent' }
\end{aligned}
$$

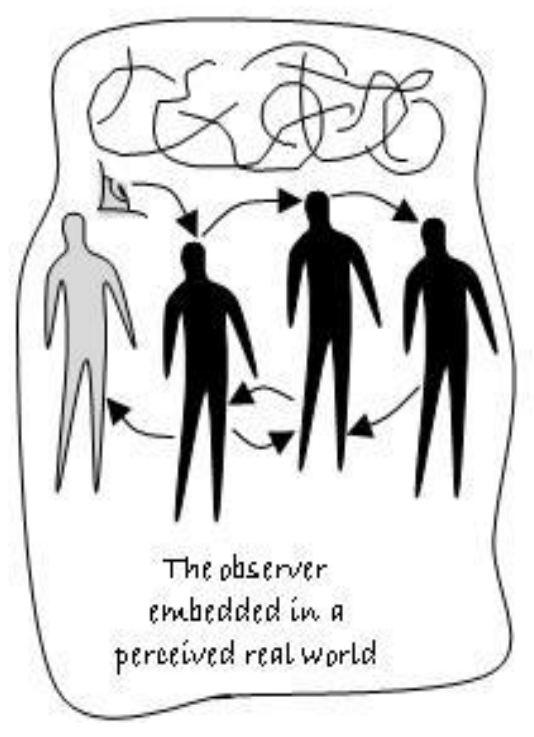

Theworld: an intereoninected social process

Figure 13 Viewing the world (adapted Checkland and Scholes, 1990 p.A11) 


\subsubsection{Positivist, Interpretivist or Critical Thinking?}

Research approaches are usually aligned with the concept of paradigms, as both frame the manner in which the world is perceived and reflect assumptions made about the research process to ultimately influence the methods of inquiry (Neuman 2006). Approaches in social research vary according to theoretical traditions, yet it is common for a combination of different elements to be used to conceptualize the problem at hand. The three main identified approaches, that of positivist social science, interpretive social science and critical social science differ in reason and nature of research (Neuman 2006) (seeTable 2), and when selected to guide methodology, will both frame how the research is represented and influence the perception of the phenomena under study (Cicmil 2006).

As noted, project management research and theory has predominantly been framed by HST typical of the traditions traceable to the engineering, construction and IT industries (Morris 2002; Winter and Checkland 2003; Crawford, Pollack et al. 2006). The focus on rational, predictable and linear models of project management (Lundin and Söderholm 1995; Winter, Smith et al. 2006; CookeDavies, Cicmil et al. 2007), “...has developed as an essentially purposeful, functionalist activity, aligning with the hard paradigm in terms of tendencies towards positivist and realist philosophies, an emphasis on objectivity, and a focus on reductionist techniques and control" (Pollack 2007 p.268) (Figure 14).

As project practice is moving across different professional and industry boundaries and an increase in complexity is being evidenced in environments that involve webs of dynamic and changing interrelationships, the certainty which traditional positivist approaches in project management research adequately catered for, is now dissipating. Calls for new paradigmatic approaches to move beyond the mechanistic and normative as dominated by HST are emerging in the literature (Williams 1999; Cooke-Davies, Cicmil et al. 2007; Pollack 2007; Whitty and Maylor 2007). There is a "...need to challenge the assumption that the rational deterministic model is an all-encompassing model of projects and project management....and ....the assumption that the deterministic model is the actual reality..." (Winter, Smith et al. 2006 p.643). The complex nature of projects, where projects are portrayed as a social process involving human activity, demand alternate paradigms and different research approaches encompassing sociological frameworks that facilitate interpretive and critical approaches to viewing the nature of social reality as supported by SST (Cicmil 2006; Cicmil, Williams et al. 2006; Pundir, Ganapathy et al. 2007).

Critical social theory, which defines social science as "...a critical process of inquiry that goes beyond surface illusions to uncover the real structures in the material world in order to help people change conditions and build a better world for themselves" (Neuman 2006 p.95), may be used as a stand- 
alone approach or combined with elements of interpretive social science, to challenge logiconormative ways of viewing and accommodating the complex world of project practice. A number of different approaches or styles of critical science are suggested, such as found in postmodernist or feminist thinking. However, perhaps the characteristics which make critical science theory most useful in transformational research, is its potential to "...smash myths and empower people to change society" and supply "...people with tools needed to change the world" (Neuman 2006 p.105). Habermas's (1981) theory of communicative action exemplifies such thinking, presenting knowledge as an emancipatory enabler affected through active self-reflection, and introducing critical knowledge as a paradigm. His hermeneutical epistemology helped close the gap between literary and social critical theory, with social ecology theory as have postmodernism and feminism, moving beyond the positivist and interpretivist approaches to understand the world.
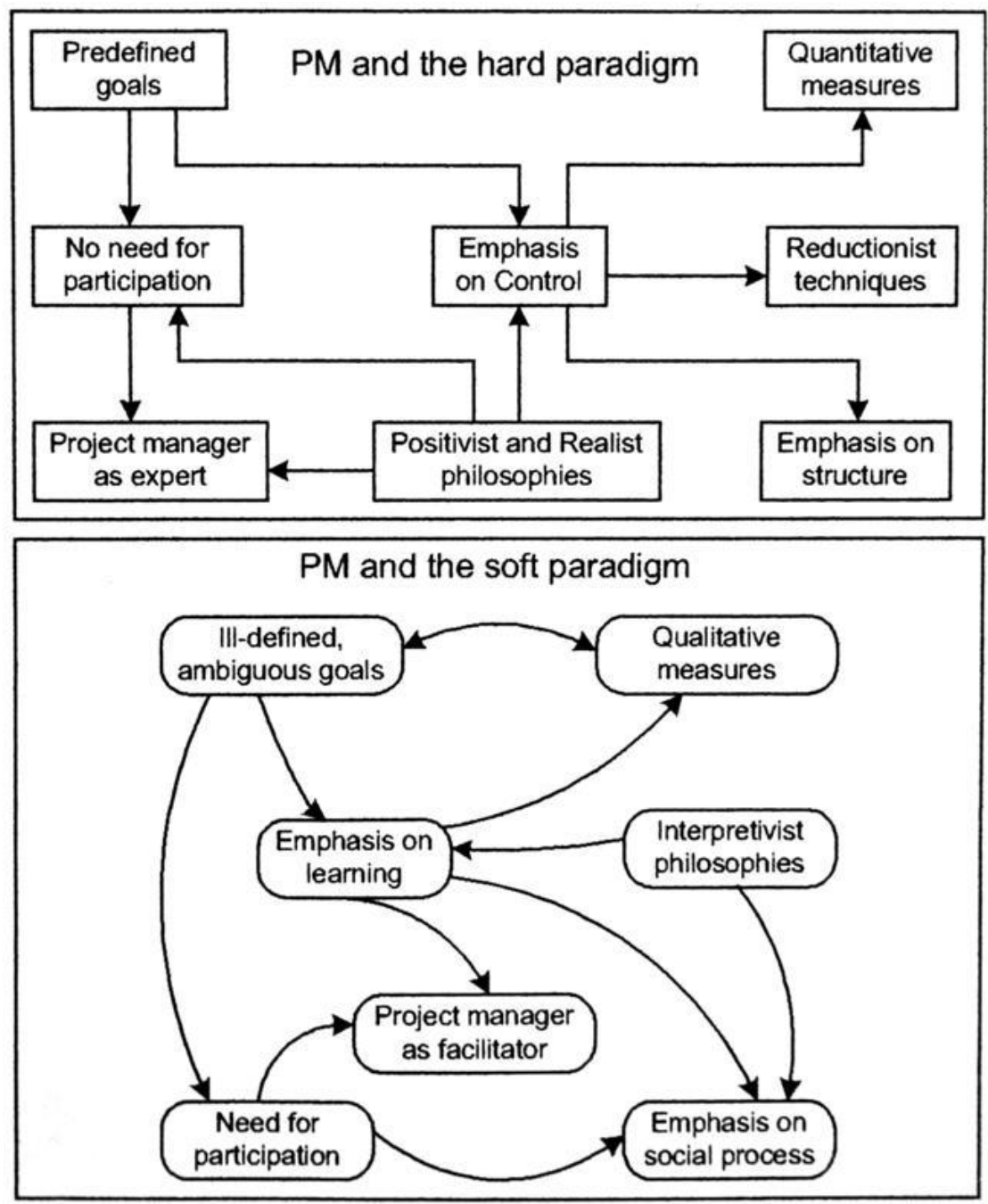

Figure 14 Project Management and paradigms (sourced Pollack, 2007 p.267) 
Table 3 Comparing Enactivism with other dominant approaches to social research (adapted Neuman, 2006 p.105)

\begin{tabular}{|c|c|c|c|c|c|c|}
\hline & & Positivism & $\begin{array}{l}\text { Interpretive Social } \\
\text { Science }\end{array}$ & $\begin{array}{l}\text { Critical Social } \\
\text { Science }\end{array}$ & Postmodernism & Enactivism \\
\hline 1. & $\begin{array}{l}\text { Reason for } \\
\text { research }\end{array}$ & $\begin{array}{l}\text { To discover natural } \\
\text { laws so people can } \\
\text { predict and control } \\
\text { events }\end{array}$ & $\begin{array}{l}\text { To understand \& } \\
\text { describe meaningful } \\
\text { social action }\end{array}$ & $\begin{array}{l}\text { To smash myths } \\
\text { \& empower } \\
\text { people to change } \\
\text { society }\end{array}$ & $\begin{array}{l}\text { To express the } \\
\text { subjective self, to } \\
\text { be playful, \& to } \\
\text { entertain \& } \\
\text { stimulate }\end{array}$ & $\begin{array}{l}\text { Learning through } \\
\text { agreed action to } \\
\text { improve a situation }\end{array}$ \\
\hline 2. & $\begin{array}{l}\text { Nature of } \\
\text { social reality }\end{array}$ & $\begin{array}{l}\text { Stable pre-existing } \\
\text { patterns or order } \\
\text { that can be } \\
\text { discovered }\end{array}$ & $\begin{array}{l}\text { Fluid definitions of a } \\
\text { situation created by } \\
\text { human interaction }\end{array}$ & $\begin{array}{l}\text { Multiple layers \& } \\
\text { governed by } \\
\text { hidden, } \\
\text { underlying } \\
\text { structures }\end{array}$ & $\begin{array}{l}\text { Chaotic \& fluid } \\
\text { without real } \\
\text { patterns or master } \\
\text { plan }\end{array}$ & $\begin{array}{l}\text { World of meaning } \\
\text { brought forth; } \\
\text { elimination of } \\
\text { subject/object } \\
\text { dichotomy }\end{array}$ \\
\hline 3. & $\begin{array}{l}\text { Human } \\
\text { nature }\end{array}$ & $\begin{array}{l}\text { Self-interested and } \\
\text { rational individuals } \\
\text { who are shaped by } \\
\text { external forces }\end{array}$ & $\begin{array}{l}\text { Social beings who } \\
\text { create meaning \& who } \\
\text { constantly make sense } \\
\text { of their worlds }\end{array}$ & $\begin{array}{l}\text { Creative, } \\
\text { adaptive people } \\
\text { with unrealized } \\
\text { potential, } \\
\text { trapped by } \\
\text { illusion }\end{array}$ & $\begin{array}{l}\text { Creative, dynamic } \\
\text { beings with } \\
\text { unrealized } \\
\text { potential }\end{array}$ & $\begin{array}{l}\text { Interconnected, } \\
\text { co-emergent, } \\
\text { embodied, } \\
\text { culturally } \\
\text { patterned }\end{array}$ \\
\hline 4. & $\begin{array}{l}\text { Human } \\
\text { agency }\end{array}$ & $\begin{array}{l}\text { Powerful external } \\
\text { social pressures } \\
\text { shape people's } \\
\text { actions; free will is } \\
\text { largely illusion }\end{array}$ & $\begin{array}{l}\text { People have significant } \\
\text { volition; they develop } \\
\text { meanings \& have } \\
\text { freedom to make } \\
\text { choices }\end{array}$ & $\begin{array}{l}\text { Bounded } \\
\text { autonomy \& free } \\
\text { choice } \\
\text { structurally } \\
\text { limited, but the } \\
\text { limits can be } \\
\text { moved }\end{array}$ & $\begin{array}{l}\text { People have great } \\
\text { volition, \& all } \\
\text { structures are } \\
\text { illusionary }\end{array}$ & $\begin{array}{l}\text { Knowing agency } \\
\text { emerges from \& is } \\
\text { nested in other } \\
\text { complex systems; } \\
\text { coupled } \\
\text { relationships }\end{array}$ \\
\hline 5. & $\begin{array}{l}\text { Role of } \\
\text { common } \\
\text { sense }\end{array}$ & $\begin{array}{l}\text { Clearly distinct from } \\
\& \text { less valid than } \\
\text { science }\end{array}$ & $\begin{array}{l}\text { Powerful everyday } \\
\text { theories used by } \\
\text { ordinary people }\end{array}$ & $\begin{array}{l}\text { False beliefs that } \\
\text { hide power \& } \\
\text { objective } \\
\text { conditions }\end{array}$ & $\begin{array}{l}\text { The essence of } \\
\text { social reality that } \\
\text { is superior to } \\
\text { scientific or } \\
\text { bureaucratic } \\
\text { forms of reasoning }\end{array}$ & $\begin{array}{l}\text { Sense-making thru } \\
\text { interactions } \\
\text { modifying \& } \\
\text { influencing } \\
\text { perceptions \& } \\
\text { understanding }\end{array}$ \\
\hline 6. & $\begin{array}{l}\text { Theory looks } \\
\text { like }\end{array}$ & $\begin{array}{l}\text { A logical, deductive } \\
\text { system of } \\
\text { interconnected } \\
\text { definitions, axioms, \& } \\
\text { laws }\end{array}$ & $\begin{array}{l}\text { A description of how a } \\
\text { group's meaning } \\
\text { system is generated \& } \\
\text { sustained }\end{array}$ & $\begin{array}{l}\text { A critique that } \\
\text { reveals true } \\
\text { conditions \& } \\
\text { helps people see } \\
\text { the way to a } \\
\text { better world }\end{array}$ & $\begin{array}{l}\text { A performance or } \\
\text { work of artistic } \\
\text { expression that } \\
\text { can amuse, shock, } \\
\text { or stimulate } \\
\text { others }\end{array}$ & $\begin{array}{l}\text { Biological/cognitive } \\
\text { theory, autopoietic } \\
\text { systems interacting } \\
\text { with environment }\end{array}$ \\
\hline 7. & $\begin{array}{l}\text { An } \\
\text { explanation } \\
\text { that is true }\end{array}$ & $\begin{array}{l}\text { Is logically connected } \\
\text { to laws \& based on } \\
\text { facts }\end{array}$ & $\begin{array}{l}\text { Resonates or feels right } \\
\text { to those who are being } \\
\text { studied }\end{array}$ & $\begin{array}{l}\text { Supplies people } \\
\text { with tools } \\
\text { needed to } \\
\text { change the world }\end{array}$ & $\begin{array}{l}\text { No one } \\
\text { explanation is } \\
\text { more true; all are } \\
\text { true for those who } \\
\text { accept them }\end{array}$ & $\begin{array}{l}\text { No absolute truth. } \\
\text { Locus of } \\
\text { knowledge inheres } \\
\text { in social } \\
\text { relatedness }\end{array}$ \\
\hline 8. & $\begin{array}{l}\text { Good } \\
\text { evidence }\end{array}$ & $\begin{array}{l}\text { Is based on precise } \\
\text { observations that } \\
\text { others can repeat }\end{array}$ & $\begin{array}{l}\text { Is embedded in the } \\
\text { context of fluid social } \\
\text { interactions }\end{array}$ & $\begin{array}{l}\text { Is informed by a } \\
\text { theory that } \\
\text { penetrates the } \\
\text { surface level }\end{array}$ & $\begin{array}{l}\text { Has aesthetic } \\
\text { properties \& } \\
\text { resonates with } \\
\text { people's inner } \\
\text { feelings }\end{array}$ & $\begin{array}{l}\text { Is experience - at } \\
\text { centre of social \& } \\
\text { cognitive sciences }\end{array}$ \\
\hline 9. & $\begin{array}{l}\text { Relevance of } \\
\text { knowledge }\end{array}$ & $\begin{array}{l}\text { An instrumental } \\
\text { orientation is used; } \\
\text { knowledge enables } \\
\text { people to master \& } \\
\text { control events }\end{array}$ & $\begin{array}{l}\text { A practical orientation } \\
\text { is used; knowledge } \\
\text { helps us } \\
\text { embrace/share } \\
\text { empathetically others' } \\
\text { life worlds \& } \\
\text { experiences }\end{array}$ & $\begin{array}{l}\text { A dialectical } \\
\text { orientation is } \\
\text { used; knowledge } \\
\text { lets people see \& } \\
\text { alter deeper } \\
\text { structures }\end{array}$ & $\begin{array}{l}\text { Former knowledge } \\
\text { has no special } \\
\text { value; it can } \\
\text { amuse or bring } \\
\text { personal } \\
\text { enjoyment }\end{array}$ & $\begin{array}{l}\text { Knowledge is the } \\
\text { space of } \\
\text { emergence where } \\
\text { knower \& known } \\
\text { meet; pragmatic } \\
\text { epistemology }\end{array}$ \\
\hline 10. & $\begin{array}{l}\text { Place for } \\
\text { values }\end{array}$ & $\begin{array}{l}\text { Science is value free } \\
\& \text { values have no } \\
\text { place except when } \\
\text { choosing a topic }\end{array}$ & $\begin{array}{l}\text { Values are an integral } \\
\text { part of social life: no } \\
\text { group's values are } \\
\text { wrong, only different }\end{array}$ & $\begin{array}{l}\text { All science must } \\
\text { begin with a } \\
\text { value position; } \\
\text { some positions } \\
\text { are right, some } \\
\text { are wrong }\end{array}$ & $\begin{array}{l}\text { Values are integral } \\
\text { to research but all } \\
\text { value positions are } \\
\text { equal }\end{array}$ & $\begin{array}{l}\text { Value is from } \\
\text { researcher being } \\
\text { attentive, } \\
\text { intelligent, } \\
\text { reasonable, } \\
\text { responsible }\end{array}$ \\
\hline
\end{tabular}


Enactivism as a theory of cognition also critically challenges positivist approaches to knowledge creation and maybe seen as a paradigm which having informed the conceptual framework of this research dissertation, is supported by a pragmatic epistemology and becoming ontology to represent a particular view of the world. An enactivist approach supports the autopoietic nature of the project as a living human activity system involving a web of interrelationships, with cognition as an emergent process inseparable from embodiment of the system (Varela, Thompson et al. 1991; Baerveldt and Verheggen 1999). As suggested by Mingers (1990a), “...critical realism is perhaps the most useful interpretation of autopoietic theory" (Kay 2001 p.469). Critical realism has also been identified by Coghlan (2007a) as underpinning authentic praxis-reflection methodology as developed by Bernard Lonergan, where action is taken through experiencing a situation and questioning what and why, and deciding what best action to take.

Enactivism, as for postmodernism, opposes positivism and builds upon both interpretive and critical social science to create a view of knowledge and the world that more ably accommodates for complexity. Postmodernism attempts to 'dismantle' social science through disputing the presence of a particular metanarrative to define reality, thus is useful for the interpretation of chaotic human realities, the power-plays which exist in social contexts, the positioning of the researcher in the research process and the questioning of all things familiar. But the tendency for postmodernism to present "...research results in a detached and neutral way" (Neuman 2006 p.104) will tend to raise, rather than resolve specific questions at hand thus is less useful in problem structuring. Enactivism has not been defined as a research methodology per se in the literature, rather is portrayed as a 'theory of the mind' drawing upon elements of Maturana and Varela's theory of biological cognition (1980), Vygotsky's (1986) Activity Theory and distributed cognition, and Wittgenstein's (1953) philosophy of language. Enactivism therefore as a theory of meaning is considered a useful research paradigmatic approach to explain consciousness, communication and cognition, to move beyond the radical constructivist view of cognition to one where cognition as embodied is inseparable from a system (Baerveldt and Verheggen 1999). Enactivist thinking when integrated with a critical theory approach to research supports active enquiry into a problem situation. The assumptions that underpin enactivism have been identified and incorporated into Table 3 adapted from Neuman (2006), to provide a comparison between dominant social research approaches and enactivist thought applied in this research dissertation.

Critical social science therefore is seen as a research approach that is potentially transformative, accommodating for a social-ecological or enactivist view of projects as autopoietic living systems, where cognition is an emergent process. Although support for critical social science approaches have been predominantly in the realm of community activist or political-social activist groups, if the solutions to new project management challenges lie in new and evolving conceptual frameworks, a 
critical approach combined with enactivist theory and postmodern thinking can offer new insight into project practice and research. This 'critical pluralism' supports Mingers' (2001) suggestion encompassing the "...need to develop new paradigms, with their own assumptions and commitments, which draw on the strengths and weaknesses of the current ones, recognizing the plurality and diversity of the world" (p.243).

\subsection{The research conceptual framework}

A research-philosophical conceptual framework has been defined for this research dissertation to incorporate postmodern thinking and enactivist theory which builds upon critical social science, to privilege a becoming ontology and a pragmatic epistemology for project practice and research, with SSM selected as the research approach. (Figure 4)

Enactivism as a theory of the mind and/or cognition has it primary assumptions being drawn from: Merleau-Ponty's (1962) phenomenological philosophical perspective of 'double embodiment'; Bateson's (1979) concept of 'mental process' as involving interconnections, external energy and circular, non-linear patterns of causality; Maturana and Varela's (1980) systems theory of cognition where living beings are identified as inherently being autopoietic; and most importantly, Varela, Thompson et al's (1991) neuro-phenomenological approach to consciousness where 'enaction' or 'embodied action' results from a continual bringing forth of a world. These theories present the mind as a process associated with living systems, as opposed to a 'thing' capable of independent thought and it is these assumptions, amongst others, which underpin the broader philosophical theory of enactivism. As a cognitive theory, enactivism draws on both radical constructivist and socio-cultural learning theories, and although scholars associated with these latter two theoretical dimensions made inroads in recognizing the importance of the context of individual learning, they fall short in explicating the embedded and inseparable nature of all life-elements within interconnected ecosystems. Enactivism as a learning theory and methodology has the potential to do this, as it "...views learning and knowing as complex, emergent processes by which dynamic agents maintain fitness with one another and within dynamic contexts...the knowing agency emerges from and is nested in other complex systems" (Begg 2002 p.4). As Reid (1996) states, "...knowing is being is doing..." (p. 208) and although radical constructivism and socio-cultural theories make reference to these different elements of learning, it is enactivism which seems to provide a more relevant theoretical framework in which to consider the instability and uncertainty of future knowing (Barnett 2004).

Enactivism assumes cognition is inseparable from embodiment, with self-reference determining perceptions of reality and environmental factors acting as triggers for system changes (Varela, 
Thompson et al. 1991). Such thinking expands upon the sociology of Husserl and Schutz which Checkland and Scholes (1990) suggest lie in harmony with SSM, through considering the nature of reality as being an embodied experience rather than one external to self. SSM is based on the premise that social reality is a result of individuals perceiving and interpreting a world 'outside' themselves (Checkland and Scholes 1990). Whilst SSM provides a useful methodology for social inquiry to bring about improvement and change, the research described in this dissertation takes a more critical enactivist approach to the nature of cognition and human existence, noting the inseparable relationships knower and known, researcher and researched, have within an environmental context.

Postmodern thinking lies in contrast to the logical reasoning of modernism and allows for the researcher to be an obvious presence throughout the research process. Postmodernism is also a useful visual tool to appreciate the dynamics of human interrelationships whose complexity is beyond the realm of control, supporting the notion of chaotic social realities sitting outside positivist scientific explanations of reasoning (Neuman 2006). Such thinking, which Pettigrew (2001a) has called - the era 'after Modernism' - contributes to the conceptual framework through reflecting "...the more complex, dynamic and internationally conscious world we live in" with there being "... a recognition that any search for general patterns should give much greater significance to temporal and spatial context" (S66). Pettigrew promotes the concepts of 'embeddedness' which is a cognitive concept pivotal to enactivist theory and 'user engagement' which is integral to Soft Systems Methodology (SSM). His call for new scholarly routes for the realities of contextualizing the creation of 'knowledge' is supported by Johannisson's (2008a) definition of 'local knowledge' in relation to enacted research as a "...physical space where the mental and social worlds are interactively produced" (p.4). The assumed embedded nature of cognition therefore involves interpersonal interconnections in a dynamic environment where an adaptive balance between an ontology of being (stability) and an ontology of becoming (change) co-exist (Johannisson, Ramírez et al. 2002; Lineham and Kavanagh 2006), much alike the evolving nature of dissipative structures in our complex universe (Prigogine 1967).

The philosophical framework designed supports praxis-based theory where projects are seen as complex, non-linear and involving multiple realities (Cicmil, Williams et al. 2006) moving away from project instrumentality to reflect a more contemporary way of thinking about projects as social process (Winter, Smith et al. 2006). As the selected research methodology, SSM is able to accommodate the generation of pragmatic knowledge by facilitating a process of social enquiry into a messy situation in a state of 'flux' and continual movement. SSM facilitates problem structuring, particularly at the front-end of projects (Winter 2006), with iterative cycles of action learning working through social complexity to facilitate sense-making for organisational transformation and resilience. 
The imperative to embrace different approaches to project management research to consider the human element and the role actors play in context, parallel the calls for more pluralistic research methods to meet the 'plurality and diversity of the world' (Mingers 2001). As Cicmil et al (2006) also have noted, "...philosophically oriented research and writing in project management... is a good way to build a more pluralist community of researchers..." (p. 677).

Viewing projects as complex living systems or as human activity systems helps move project conceptualisation closer to the non-linear and non-static actuality of project practice. As Pettigrew (2001a) has identified, there is a need for management research to move beyond the modernist assumptions and 'bi-polar' thinking dominating mechanistic, positivist frameworks. "...the complexity and uncertainty of the knowledge production process demand of us the exploration of many different types of knowledge production, user engagement and mechanisms of impact" (p.S62). The defined conceptual framework and epistemology aims to explore multiple world views to make sense or bring forth a world of meaning to facilitate emergent cognition in projects. SSM as a process of social inquiry bridges the divide identified by Pettigrew, through involving the researcher in the change process, where a series of learning cycles involving purposeful action act as the enabler of social process (Checkland and Scholes 1990). Enactivism as a framing theory, then extends upon the constructivist view of an world external, to present the notion of embodied cognition emergent through interactions.

A pragmatic epistemology acknowledges the interconnections between action and knowing, supporting an ontology of movement and flow. The philosophical traditions of pragmatism lie in the works of Charles Peirce (1868), William James (1975) and John Dewey (1929) where the concept of 'knowledge for action' is based on the assumption that "...knowledge rests upon an active will of individual agency that is constantly exercised" (Meyers 2005 p.3) (see Section 2.3.2). A pragmatic epistemology assumes the life worlds of individuals entail an interconnect with phronesis or practical knowledge coalescing with reflective practice or 'knowledge about' (Spender 1996b), to support a moral appreciation of a local context and associated realities (Flyvbjerg 2001). Human existence is seen as embedded in context with knowledge emerging from social interrelationships coupled with each other and the environment, where learning is a social activity arising from interactions and active sharing of experience involving mutual reciprocity (Maturana and Varela 1980; Lave and Wenger 1991; Varela, Thompson et al. 1991; Capra 1997). A pragmatic epistemology aligns pragmatic forms of inquiry with holistic approaches to cognition, as opposed to reductionist thought and passive concepts of learning, through emphasising the social nature of learning and the active sharing of experience (Courtney, Chae et al. 2000). Such an approach moves beyond the Absolutist claims of a finite truth and the Skeptics who dispute that a belief could ever be true, to justifiable beliefs which may be considered 'true' (Cavaleri, Seivert et al. 2005). 
Pragmatic knowledge as emergent through action and reflection informs various applications of action learning. As suggested by DeFillippi (2001), these encompass: interactions between individual reflective interpretations of experience and the explicit instruction and imparting of 'knowledge' in more formal educational and business settings; a science which investigates underlying assumptions which affect learning, particularly in groups or organisational settings (Argyris and Schön 1996); a research approach, introduced by Kurt Lewin in the 1930s where it was suggested that the researcher becomes actively involved in the situation to increase the plausibility and understanding of social situations (Checkland and Poulter 2006); and project-based learning involving the social context in and through communities of practice (Wenger 1999). It is the science and research approach in particular which are relevant to the conceptual framework described in this dissertation. The assumed interconnect between researcher and context, where space and time are shared and action-reflection underpins emergent knowledge, reflect a pragmatic epistemology where individuals are engaged with one another in context to bring forth a world of meaning (Husserl 1931; MerlauPonty 1962; Schutz 1967; Maturana and Varela 1980; Calori 2002 ; Checkland and Poulter 2006).

An ontology of becoming is the new reality as reflected in the emergent nature of cognition, where meaning is created through circular processes involving 'knower and known', relationships involve mutual reciprocity and "...knowledge is ever-evolving and changing..." (Proulx 2008). The significance of causality, structure and the properties of entities begin to fade, as a world of action, interrelationships and processes shift thinking from an ontology of being to an ontology of becoming (Chia 1995). Such thinking privileges a world viewed as patterned and complex, non-static, nonlinear and evolving; one that aims for resilience through processes involving adaptive responses to environmental triggers. Consciousness arises through a world comprising patterned interconnections, with living entities being dynamic systems capable of self-regulation and autonomous reproduction (Varela, Maturana et al. 1974; Maturana 1999). Organizations as complex adaptive systems involve cognitive processes which facilitate change through learning arising from dialogue (Hall 2003). Projects as social process involving human activity, are better perceived as emergent, involving interactions to better cater for the complex nature of today's business world. Post modern thinking supports the concept of "...action, movement, process and emergence...", catering for "...paradox, uncertainty and the not yet known..." (Chia 1995 p.597). A becoming ontology involving co-adaption and co-emergence better accommodates for challenges being faced in an era grappling with social complexity as reflected in project practice (Cooke-Davies, Cicmil et al. 2007); whilst enactivism as a theory of cognition providing the socio-philosophical assumptions that support the conceptual framework guiding this research exploration. 


\subsection{The choice of research approach}

Although it has been suggested by Neuman (2006) that an 'approach' and a 'paradigm' are similar due to representing particular assumptions about how the world is perceived, it is suggested that a paradigm provides the broader overarching 'thinking' framework into which a research approach 'fits', so that suitable data collection techniques and methodological approaches can be selected to complement.

\subsubsection{Evaluation, Action or Impact assessment?}

Applied social research aims to find solutions to a particular social problem, with evaluation, action and impact assessments being identified as the three main types of research commonly used (Neuman 2006). Evaluation research typically assesses both ongoing progress and final outcomes of a program or policy and is useful if the goals are well-defined and standards of measurement are clear; whereas social impact assessment research usually involves evaluating a program which may have had clear goals and predictions in the outset, but the consequences of the implementation were uncertain. In both of these approaches, assessment or evaluations may provide information that initiates change in strategies next time round, but the overall initial research aims are directed at assessment rather than social change.

Action research, however, is "...applied research in which the primary goal is to facilitate social change or bring about a value-oriented political-social goal" (Neuman 2006 p.28). Problematic situations in socially complex situations which involve disparate cultural orientations within a project organisation, require action that moves beyond identifying and analysing problems, to facilitate knowledge creation for organisational transformation. Social science enquiry lies in the human domain of research practice. The certainty of repeatable findings underpinning 'scientific knowledge' is not practical or realistic in situations involving the presence of human realities in constantly changing environments and social complexes (Checkland and Scholes 1990). Involvement of the researcher in context where the "...researcher and researched 'walk the path together'..." (Calori 2002 p.879), necessitates a methodology where action and reflection are interconnected through streams of consciousness being entwined in intersubjective relationships sharing a community space in everyday life (Husserl 1931; Schutz 1967). Experience becomes the 'social reality' and communication occurs in consensual domains where language as the phenomenon arises from individuals being structurally coupled with one another (Maturana 1999).

Action research has also been identified as one of three main research strategies involving the researcher embedded in context, with ethnographic studies and enactive research being two others 
which involve action-reflection (Calori 2002). Researchers involved in ethnographic studies interpret or describe cultural aspects of social groups as they perceive the social context in which they are embedded as an observer (Neuman 2006; Johannisson 2008b). Qualitative rich descriptions are usually used to facilitate multiple interpretations, but in essence the aim is for the reader to feel they are there, experiencing the same realities (Geertz 1973). Enactive research also involves the writer or researcher, sometimes retrospectively reporting or describing a phenomenon in which they have been dynamically involved over a long period of time (Calori 2002). This appears as a form of field research where the researcher "...examines social meanings and grasps multiple perspectives in natural social settings" (Neuman 2006 p.383). Both ethnographic studies and enactive research involve subjective interpretation of social contexts, limiting readers understanding to a subjective interpretation to portray events or happenings. Experience is primarily the researcher's experience, and although the researcher's pre-conceptions need to be identified, the purposes of ethnographies and enactive research are usually to describe or report on situations which have taken place from an insider observer's perspective.

Enactive research as proposed by Johannisson (2008b) also involves the researcher initiating a change in a context, recognizing reality as structured and institutionalized, adopting a social constructionist view, orchestrating and making emergence intelligible, being involved in construction of new realities, and completing multiple writings (p. 7-8). This approach shares similar thinking with SSM, with enactive research having both pragmatism and phenomenology underpinning views of reality. The enactive methodology moves beyond self-ethnographic representations of "...processes, existing structures or external events produce" to facilitating the "...birth of a new project but accepting its emergence as a partially uncontrolled outcomes of interactive processes, incorporating existing structures as well as external events" (Johannisson 2008b p.8). The enactive research methodology is a type of 'practice-theory' approach that has been used in relation to entrepreneurship research to investigate different phenomenon. Whilst not as 'established' as SSM, the concepts underpinning an 'organizing context' as interlinked with an enacted social environment reflect the assumed interconnected and interactive nature of human realities and the centrality of experience to emergent entities.

The research described in this dissertation however, selected SSM as the research methodology, with a number of purposes in mind. The rationale will be further discussed in section 3.6, but one benefit SSM shares with ethnography and enactive research, is that it supports an action-reflection relationship of 'researcher and researched' immersed in context. SSM however, moves beyond these research strategies to construct a learning system as a social process to facilitate feasible and desirable change through actively seeking solutions to messy, ill-structured problems (Checkland and Scholes 1990; Yurtseven 1999). The interventionist nature of action research facilitates 'actionable 
knowledge' through cognition emerging as a process of learning, integrating action with reflection. "Action research requires involvement in a problem situation and a readiness to use the experience itself as a research object about which lessons can be learned by conscious reflection" (Checkland and Scholes 1990 p.16). Action research has been accused of aspiring to 'democracy in research' (Johannisson 2008b) through attempting to facilitate mutual understandings in practice, which phenomenologically would be challenging. However, SSM is more accurately portrayed as using the theory of action research within a declared language framework to explain how knowledge will be defined (Checkland and Poulter 2006). SSM has the researcher taking part in the action, with the research being participative, qualitative and involving iterative cycles of planning, action, observation and reflection for mutual learning (Argyris and Schön 1996; Raelin 2000; Levin 2003; Checkland and Poulter 2006; Coghlan 2007b; Sankaran, Hou et al. 2009).

SSM is a structured methodological approach accommodates for phronesis as "practical wisdom" (Flyvbjerg 2001) within a framework of soft systems thinking to merge normative and descriptive research strands in order to more comprehensively address complex contextualised problems (Checkland and Scholes 1990). Action research involves diagnosing, planning, taking action and evaluating, plus a 'pre-stage' step which focuses on the context and purpose of the research intervention (Coghlan and Brannick 2001). This research dissertation has used SSM for its action research approach to bring about improvement in a human problematical situation, with an emphasis on learning through acknowledging the realities of emergent cognition as a social process. Enactivism as the paradigm and language guiding this research, moves beyond social constructivist and radical constructivist theories (as influencing enactive research and informing SSM) to support the view of cognition as embodied and emergent, with researcher and researched sharing a relational reciprocity for making sense of the world.

\subsubsection{Qualitative or quantitative?}

The empirical evidence required to confirm, measure or explain the social world involves differing approaches to data collection. Noting the calls to expand the project management theoretical base, together with the challenges faced in socially complex environments, a qualitative approach which acknowledges the researcher's presence of self, was considered more likely to allow for new and emergent knowledge creation than would a quantitative approach (Checkland and Scholes 1990; Neuman 2006; Coghlan 2007a). The social nature of the research problem required a suitable strategy to not only decipher the situation, but to bring about change necessary to improve processes in project implementation. Having aligned thinking with soft systems within an enactivist paradigm for reasons as discussed in 3.2 , and noting the interactive nature of projects as social 
process, the action methodology required a complementary approach for collecting and analysing the data. The nature of the data was primarily 'soft data' being drawn from interviews, discussions and meetings, involving pictorial representations as 'rich pictures' and analyses which involved reflections upon the social, political and culture context. However, although a qualitative approach (which is inherent to SSM) was the dominant orientation chosen so data could be easily extracted despite situational constraints and social complexities, elements of quantitative analysis were incorporated to gain a richer understanding of the processes involved in the problematic situation.

Quantitative methods applied as a singular strategy in data collection and analysis, tend to atomise knowledge by measuring objective facts and removing the subjective elements in enquiry through separating the problem from the context. This is typical of the broader concept of "hard systems thinking' (HST) or hard paradigm, where epistemology is aligned with positivism and linear models of goal directed behavior dominate enquiry. In contrast, a soft paradigmatic approach involving 'soft systems thinking' (SST), is more commonly associated with interpretive or critical social science thinking, where the contextual nature of exploratory research seeks to find the processes of knowledge as opposed to a universal truth (Winter and Checkland 2003; Reisman and Oral 2005; Cicmil 2006; Pollack 2007). Given the social nature of the problematic situation where the embeddedness of project-in-context was considered pivotal to the enquiry, together with my central role as researcher to the investigation, a qualitative approach incorporating a pragmatic epistemology, was considered more able to explore the social realities and cultural meanings.

A qualitative (or quantitative) approach involves assumptions which influence research orientation, research design, methods of measurement, sampling and analysis (Neuman 2006). The research in this dissertation takes a critical approach and non-linear research path to investigate an ill-defined complex problem, applying 'logic-in-practice' to draw upon multiple interpretations of contextual issues. A purely quantitative approach would necessitate more 'known' factors so that logic could be reconstructured, testable and objectively quantified. Despite the researcher's experience and knowledge of the situation, open-ended, vague, unstructured questioning was utilized, particularly at the onset, to uncover new realities and keep options open for the direction of the research. $A$ quantitative approach would have been more appropriate if there were clear questions which needed to be answered, with a pre-planned focus rather than emergent (Neuman 2006). Nevertheless, whilst narrative and rich pictures dominate data presentation and exploration, it was found useful to sort interview data in thematic sequence to quantify main areas of concern to help focus discussions. This combined approach portrayed individual and group perceptions as models of activity, whilst clustering similar ideas (App. 7). “...different research methods (especially from different paradigms) focus on different aspects of reality and therefore a richer understanding of a research topic will be gained by combining several methods together..." (Mingers 2001 p.241) 
SSM involves a research methodological design which clarifies the 'fuzz' through rich picture 'descriptions' of individually perceived contextual problems. SSM could be said to involve the skills of a qualitative 'bricoleur', through compiling pictorial representations of individual and group perceptions of a problem situation to compare conceptual models with real-world models. The interpretative process involves using these pictures to explore the lifeworlds of individuals and build conceptual models to make sense of the data and gain a sense of how others perceive the world and ascribe meaning to the situation.

Quantitative research would propose causal hypotheses and relations, with an aim to 'solve' the problem situation through focussing on converting definable variables into action and measuring data, usually numerically and separate from analysis. Qualitative research involves ongoing analysis through the data collection process, where data more commonly involves narrative, description, pictures and symbols rather than numbers. "The qualitative researcher reexamines and reflects on the data and concepts simultaneously and interactively" (Neuman 2006p.181). SSM is a "systemic learning process' building upon Vicker's (1965) work, cited in Checkland and Sholes (1990) on 'appreciative systems'. The approach moves beyond goal-directed enquiry into human systems, to qualitative enquiry valuing individual perceptions and judgments about facts and values of a world which influence decisions about relationships we establish, maintain or dissolve over time (Checkland and Scholes 1990).

SSM seeks change which is 'systemically desirable' and 'culturally feasible'. Selecting a qualitative or quantitative approach may be driven not only on the data or evidence being pursued, but may reflect the underpinning philosophical assumptions which frame the research. As Yin (2003) has suggested, "...regardless of whether one favours qualitative or quantitative research, there is a strong essential common ground between the two" (p.15). The conceptual framework described in this dissertation favours a soft systems approach to the research problem for reasons as discussed, with a qualitative approach dominating the 'measuring' of data throughout the collection process. Reflection and reexamination of concepts take place through the whole research process, generating ideas for varying treatment and directions for the research. At some stages in the iterative cycles of action in SSM, treatment of the data has benefitted from sorting in a quantifiable manner to present to stakeholders, in part due to their conceptual preferences and in part as it was perceived as useful to the research process. Also, when modelling relevant systems in the logic-driven stream, comparing conceptual models with a real-world model may warrant different treatment in the form of a matrix for conceptual purposes to lie alongside rich picture descriptions. Comparison does not aim to 'improve the models', which would be more alike HST, rather "...it is to find an accommodation between different interests in the situation, an accommodation which can be argued to constitute an improvement of the initial problem situation" (Checkland and Scholes 1990 p.44). 
What is assumed in qualitative research as guided by SSM, is that there is opportunity for open participatory debate. There is a need however, to be open to using different techniques according to stakeholder characteristics, with Jackson (1991) even suggesting that such debate "... is impossible to obtain in problem situations where there is fundamental conflict between interest groups which have access to unequal power resources" (p.133). SSM in essence is a 'combination of methods' or in actuality a methodology in itself, which is suitable for implementation in a variety of research settings and in research practice. As a 'structured set of guidelines or activities' the principles guiding SSM are able to support the generation of valid and reliable results (Mingers 2001). Given the complex nature of the research context, rather than focusing on a quantitative-qualitative divide, perhaps what may be a more useful in envisaging the approach, is to suggest that SSM provided a 'systemic' 'approach to understanding the interaction of variables' rather than an 'analytic' 'approach to understanding a few controlled variables' (Miles and Huberman 1994 p.41).

\subsubsection{The purpose - exploratory, descriptive or explanatory?}

The purpose of the research is commonly defined as being either exploratory, descriptive or explanatory with differences reflecting the focus of the problem and what the researcher hopes to achieve (Babbie 1998; Neuman 2006; Richey and Klein 2007). Again, as for research strategies and approaches, more than one purpose may exist, however one usually dominates. The identified differences are summarised in Table 4, drawing upon both Richey and Klein (2007 p.44) and Neuman (2006 p.34) to compare the main underlying factors influencing choice, with the related focus being added as of relevance to 'examining the problem'.

Explanatory research focuses on the 'why' of situations, aiming to develop or extend upon existing theory. The research commonly involves hypotheses testing to explain reasons behind a situation or event and fits comfortably within a HST paradigm. In comparison, descriptive and exploratory research share a number of similarities, with exploratory focussing on the 'what' of a situation and descriptive leaning more towards the 'how' and 'who'. As noted by Pettigrew (2001a), "management research which delivers both what-is and how-to knowledge is much more likely to meet the challenge of scholarly quality and relevance" (p.S66). Both descriptive and exploratory research frequently merge in practice to provide a more comprehensive approach, with descriptive research 'painting a picture' of the situation and exploratory research seeking to go beyond this picture to gain an understanding of what is happening. Enactivism views "... description to be constitutive of reality rather than it being a representation of pregiven world..." (Baerveldt and Verheggen 1999 p.191), with 'how' things are perceived taking precedence over 'why'. 
Investigating the relationship of projects in context as 'project ecologies' has been identified as critical to understanding the impact project practice has on shaping and changing today's business world (Söderlund 2004b). The descriptive qualities of SSM in painting a picture of a social phenomena or 'fuzzy' situation (Dodouras and James 2007) through drawing upon multiple interpretations, is combined with exploratory analyses of underlying social factors. The Logic-Based stream of enquiry found in SSM may be seen as the descriptive research strand, where the worldviews of stakeholders are used to describe particular issues and factors through ascertaining their perceptions of the problem and the 'model of purposeful activity' they describe as their reality of the situation. Exploration of the related social issues or the 'what' processes which may be involved are accommodated for by SSM in the normative or Cultural-Based analysis stream. SSM therefore bridges the normative and descriptive divides which commonly exist in organisational learning research, to provide a more holistic approach that enables organizational and project processes to inform intervention and practice (Robinson 2001).

As can also be seen in Table 4 different purposes of research have a selection of associated research methods. Case study, in-depth interviews, mixed method and content analysis are four identified methods that are identified for both exploratory and descriptive research. Given the nature of the problematic situation described in the dissertation, neither content analysis, which examines the 'content of a communication' for recording and analysis purposes, nor in-depth interviews in their traditional form involving detailed transcription for analysis, were necessarily useful. 
Table 4 Purpose and Methods of Research (adapted Neuman 2006 p34 \& Richey \& Klein 2007 p44)

\begin{tabular}{|c|c|c|}
\hline Purpose of research & Common Research Methods & Examining the problem \\
\hline \multicolumn{3}{|l|}{ Exploratory } \\
\hline $\begin{array}{l}\text { *Relates to topics about which very little is known } \\
\text { *Research design less structured } \\
\text { *Improves familiarity with basic facts, setting, and concerns } \\
\text { *Creates a general mental picture of conditions } \\
\text { *Helps formulate and focus questions for future research } \\
\text { *Generates new ideas, conjectures or hypotheses } \\
\text { *Determines the feasibility of conducting research } \\
\text { *Develops techniques for measuring and locating future } \\
\text { data } \\
\text { *Usually qualitative research } \\
\text { *Usually not connected to a specific theory or research } \\
\text { question }\end{array}$ & $\begin{array}{l}\text { Case study } \\
\text { Content Analysis } \\
\text { Field Observations } \\
\text { In-depth interview } \\
\text { Mixed method } \\
\text { Multiple methods } \\
\text { Literature review and analysis } \\
\text { Think aloud methods } \\
\text { (SSM) }\end{array}$ & Focuses on the 'what' \\
\hline \multicolumn{3}{|l|}{ Descriptive } \\
\hline $\begin{array}{l}\text { *'Fact finding' } \\
\text { * Focus on relatively few dimensions of well-defined entity } \\
\text { *Provides a detailed, highly accurate picture of a situation } \\
\text { *Assists in locating new data that contradicts past data } \\
\text { *Creates a set of categories or classification types } \\
\text { *Clarifies a sequence of steps or stages } \\
\text { *Documents a causal process or mechanism } \\
\text { *Reports on the background or context of a situation } \\
\text { *May be quantitative or qualitative }\end{array}$ & $\begin{array}{l}\text { Content analysis } \\
\text { In-depth interviews } \\
\text { Survey } \\
\text { Mixed methods } \\
\text { Case study } \\
\text { Evaluation } \\
\text { Expert review } \\
\text { (SSM) }\end{array}$ & $\begin{array}{l}\text { Focuses on the 'how' and } \\
\text { 'who' }\end{array}$ \\
\hline \multicolumn{3}{|l|}{ Explanatory } \\
\hline $\begin{array}{l}\text { *To describe phenomena \& to test relationships between } \\
\text { elements of problem } \\
\text { *Tests a theory's predictions or principles } \\
\text { *Elaborates and enriches a theory's explanation } \\
\text { *Extends a theory to new issues or topics } \\
\text { *Supports or refutes and explanation or prediction } \\
\text { *Links issues or topics with a general principle } \\
\text { *Determines which of several explanations is best } \\
\text { *Usually quantitative }\end{array}$ & $\begin{array}{l}\text { Experimental } \\
\text { Evaluation } \\
\text { Survey }\end{array}$ & $\begin{array}{l}\text { Focuses on the 'why' and } \\
\text { to a lesser extent the } \\
\text { 'how' - the 'what' is } \\
\text { usually already defined }\end{array}$ \\
\hline
\end{tabular}


Case study research as a precursor to action research may have been used to initially retrospectively study the situation, but its approach is more beneficial when data can be collected over a long period of time, which was not possible in this project. Such an approach is an "...empirical inquiry that, investigates a contemporary phenomena within its real-life context; especially when the boundaries between phenomenon and context are not clearly evident.." (Yin 2003 p.13). Case study inquiry also 'copes with technically distinctive situations with multiple variables, relies on multiple sources of evidence requiring triangulation for analyses and can benefit from prior development of theoretical propositions to guide data collection and analysis' (Yin 2003). Such research may involve the investigation of one case in-depth or several cases to compare and may be used to develop new theories through applying a grounded theory approach to case analysis (Glaser and Strauss 1967). The approach may also be applied to explain, describe illustrate, explore or meta-evaluate situations (Yin 2003). But given that long-term case writing from one perspective did not have the potential to unravel an ill-defined 'phenomena' in this research, and noting the multiple socio-cultural elements involved, SSM with its own structured yet flexible methodology was considered to be a more relevant approach to explore the complexities and bring about improvement.

A mixed method approach, which is more commonly associated with research whose purpose may be exploratory or descriptive, has the potential to focus on the 'what', 'how' and 'who' of a problem situation. Incorporating more formal inquiry processes such as surveys or formal interviews to investigate a more clearly defined problem or research question can be useful and may precede an action research approach to improve the situation. SSM as an action research approach may even be seen as incorporating elements of 'mixed method' approaches through utilising interviewing, reflective journals, large group meetings and pictorial representations of the problem at hand. SSM may also include in-depth interviews using open-ended questions to explore individual perceptions of a problematic situation, but rather than audio-taping and transcription as in phenomenological or ground theory research for example, resultant data is presented pictorially. As noted by Cicmil, Williams et al. (2006 in Sankaran and Tay 2007), given that "Real projects are increasingly tending to be complex social settings, unpredictable (ambiguous and uncertain) with control and collaborative interactions between diverse actors" (p. 12), approaches to research problems should be adaptable. SSM was seen to comprehensively incorporate descriptive logic-based analyses and normative sociocultural analyses, with qualitative descriptions being interspersed with more structured purposeful action planning. Flyvbjerg (2006) also has suggested that “...good social science is problem driven and not methodology driven in the sense that it employs those methods that for a given problematic, best help answer the research questions at hand" (p.242). It was the messy, ill-defined problem combined with the need for improvement, that underpinned selecting SSM as the methodological approach to find out 'what' was going on, 'who' could contribute to the change process and 'how' it 
all might be brought about. "SSM's value lies in identifying the problem situation in an organized manner" (Reisman and Oral 2005 p.165) and as a methodology, it moves beyond exploration and description, to facilitate action for change to improve a situation through using conceptual models which may be seen as 'hows', in turn determining the way the 'what' is expressed (Mingers 1990b; Checkland and Tsouvalis 1997).

\subsection{The research methodology}

\subsubsection{Section introduction}

In selecting or designing a methodology, Mingers (2001) has argued that “...consideration should be given to the different dimensions of a real situation, material, social and personal; to the tasks involved in the different stages of a research study; and to the research context (including the capabilities and characteristics) of the researcher(s)" (p.256). This process was followed in preparation for this research dissertation through giving detailed consideration to the real-life problematic situation being faced in context where consensus on the nature of the problem was elusive, project goals were ill-defined and a socially complex project environment involving a human plurality existed.

SSM is the methodology selected for the research described in this dissertation, with various reasons and justifications already been woven through discussions in the previous sections on choice of paradigm, conceptual framework and research strategy. The next section, 3.6.2 outlines SSM as a research methodology, summarising the value of SSM in relation to this research dissertation in project practice, 3.6.3. This is followed by 3.6 .4 which details the research method pursued.

\subsubsection{Soft Systems Methodology (SSM) - The methodology and rationale}

SSM was originally developed in response to difficulties being encountered in 'solving' more illdefined socially derived problems using traditional Systems Engineering and Systems Analysis approaches. Such methodologies were based on traditional hard-systems thinking which relied on having the 'what to do' already defined. SSM was designed as “...a methodology that provides a set of principles for intervening in human problem situations in order to bring about what will be judged to be 'improvement'" (Checkland and Winter 2006 p.1435). The historical roots of this style of systems approach have been traced to the 1960's work done by Jenkins and Optner (cited in Checkland and Scholes, 1990) where a system was defined as “...a complex grouping of human beings and machines for which there is an overall objective" (Yurtseven 1999 p.229). 
The impetus for developing a different or new approach to addressing complex and 'difficult to solve' problems in the social sciences was the failure of rigid approaches utilising HST to cater for problems that were ill-defined. Checkland is attributed with the development of SSM as an alternative methodology to address complex social science problems, basing his response on the premise that systems engineering approaches failed "...to cope with anything other than well-structured problem situations" (Checkland and Scholes 1990 p.18). His new 'soft' systems approach acknowledged the systems concept of a 'whole being greater than the sum of its parts', the emergent properties of system components, and the importance of the processes of communication and control in survival and adaptation to changing environmental contexts. Purposeful actions are seen to take place in emergent 'holons' or human activity systems, with the process of enquiry being the system, rather than the system being the object of enquiry as in HST (Checkland and Scholes 1990). SSM as a process of enquiry has two contextual components integral to the approach. One is action research with individuals utilising experience and reflection in determining intentions and purposeful action; and the other is the social process of appreciative systems based on Vicker's (1965) work where perceptions of the world are dependent upon judgements made about the world and what is valued.

The sociological assumptions underpinning SSM are based upon Husserl's essential phenomenology (1931) and Schutz's lifeworld phenomenology (1967). Husserlian phenomenology studies the human phenomena without looking at causality or objective realities, to explore how individuals construct a lifeworld of meaning through being intersubjectively connected with others in and through social activity. Schutz further expanded upon Husserl's main assumptions, to create a 'phenomenology of the social world', suggesting that in relation to social science research, "People are engaged in an ongoing process of making sense of the world in interaction with their fellows and we, as scientists, are seeking to make sense of their sense-making" (Wilson 2002 p.2).

In particular, it is the phenomenological concept of the 'meaningful construction of the social world' through these interconnections, together with 'models of human action for making sense of the world' that underpin thinking behind SSM. Knowledge, according to Schutz, develops from people experiencing a world of meaning, with "...the knowledge of the man who acts and thinks within the world of his daily life ... not [being] homogeneous; it is (1) incoherent, (2) only partially clear, and (3) not at all free from contradictions" (Schutz 1964 p.93). Socially acquired knowledge is of four types, eyewitness, the insider, the analyst and the commentator (Schutz 1964). Whilst Checkland and Scholes' (1990) SSM involves model building and purposeful human action, the underlying construct particularly at the beginning of the research, is of an observer (or 'eyewitness') perceiving a complex problem which is worthy of exploration through a process of inquiry which is the system. The researcher later becomes involved in model building and analysis but the position tends to be based on metaphysical realism and "...ontological subjectivism of [a] constructivist" (Christis 2005 p.14). 
Even the 'insider' role as identified by Schutz, is somewhat different from the 'insider action research' position, with the former being someone who can more accurately 'report' happenings within a context due to participation inside, where as insider action role connects the researcher and the researched in a position based on interventionism (Coghlan 2007a). Whilst the researcher becomes involved in the action of SSM, the multiple roles $\mathrm{s} /$ he may have as project manager, researcher and driver of action, need to leave room for multiple emergent interpretations to ensure a subjective stance does not dominate or determine project directions.

Thus, the phenomenological underpinnings of SSM are considered useful in relation to conceptualising the derivation of knowledge through human experience and interpretation. But the inclusion of enactivism in the research conceptual framework extends upon this thinking to portray knowledge as emerging through social interaction, with experience being inseparable from human activity systems which are also emergent phenomena arising from these interactions. SSM draws upon phenomenology to describe the nature of reality as comprising a world of meanings intersubjectively created. But the world is primarily viewed as being 'observable' rather than a reality as described by enactivist theory where subject and object merge as one. Phenomenology as a research method is useful in discovering the world as is experienced by others with individual meaning ascribed to a problem situation adding to the researcher's understanding or perception of the world. However, to make this knowledge actionable to instigate improvement through learning, SSM more ably facilitates change, whilst enactivism assists in emergent cognition for resilience and adaptation.

\subsubsection{SSM and project practice}

SSM finds its usefulness in project practice as its active research process enables enquiry for transformation. The capacity of SSM to be embedded in a continually evolving project context helps unravel some of the mess that exists in endeavours involving disparate human elements (Dodouras and James 2007). SSM uses a problem structuring approach as a means of social inquiry, and is particularly useful at the front-end of projects through drawing upon multiple stakeholder views to ascertain their perception of the problem at hand (Moores and Gregory 2000; Winter 2006). In selecting SSM as the research approach for this dissertation, consideration was given to the centrality of the human element in project implementation, the non-static nature of human interactions and the conflicting worldviews which exist in 'real-life' situations (Kakabadse, Kouzmin et al. 2001; Barnett 2004; Checkland and Poulter 2006). SSM was considered to have the potential capacity to investigate the complex systems involved, as the problem appeared ill defined and some improvement was deemed necessary to bring about change through project practice for 
organisational transformation. SSM as an active process, necessitates using the experiences of researcher and other stakeholders as the research object and assumes there is active involvement in a problematical situation (Checkland and Scholes 1990). Identifying projects as a social process and therefore as human activity systems, supports the notion that they serve as a 'device' to explore the world, rather than exist in the world as a rational entity (Checkland 1995).

The flexibility of SSM as a social learning process was therefore considered potentially beneficial in helping sift through project 'messes' to accommodate for complexity, through focussing on "...the process of 'managing', rather than the life-cycle process of 'project management'" (Winter and Checkland 2003 p.192). SSM has been used in this research to facilitate identification of individual stakeholders, instigate mapping processes and assist in making sense of the entire situation rather than isolated components. Given the complex and ambiguous socio-cultural research context, together with the "...fuzzy ill-defined situations involving human beings ..." (Checkland and Scholes 1990 p. 10) SSM was the most useful methodology to employ due to its cyclic nature actively seeking improvement for real-life human problems (Checkland and Poulter 2006). Dynamic environments necessitate dynamic solutions to keep pace with the adaptive responses needed to survive in today's project management environment. SSM moves beyond just describing messy situations, to facilitating consensus goal identification and action, whilst being flexible enough to accommodate for emergent change and redirection (Checkland and Poulter 2006; Dodouras and James 2007).

\subsubsection{Soft Systems Methodology - methodology or method?}

SSM is an action research approach based on social inquiry involving seven principles and five actions (Checkland and Poulter 2006), utilising experience of involvement with a real-life problem as the research object (Checkland and Scholes 1990). The principles assume identification of a 'real-world' problem, where different 'worldviews' of the problem exist together with people 'acting purposefully' and 'discussion and debate' being structured to accommodate for differences in these worldviews. These five principles underpin the ongoing learning cycle (principle six), which is complemented by the seventh principle of conscious reflective practice (Checkland and Poulter 2006). The four main actions that are defined in SSM, involve finding out about a problem situation, making models to explore the problem as informed by different world views, encouraging the questioning of the situation to find 'desirable and feasible' change, to then define and take action. Reflective practice forms the fifth action, but takes place at a different level to ensure that emergent change encompasses all four other actions and may be seen in actuality to be a concurrent process (Checkland and Poulter 2006). 
The principles underlying SSM provide a flexible framework in which to pursue action and research to move beyond more static approaches that assume a linearity in enquiry. As suggested by Checkland and Poulter (2006) SSM is a methodology rather than a 'method', as it is guided by principles as opposed to specific steps in a process. Thus although there is a framework for the research (i.e. a methodology) the approach is flexible enough to accommodate for the ebb and flow of contextual changes which may appear at random throughout the social inquiry process. This action cycles in the research described in this dissertation involved reflective practice throughout, but in particular was combined with 'taking action', having separated somewhat the defining and taking of action.

The original detailed research methodology of SSM incorporates two streams of enquiry and analyses, the logic-based and cultural streams as portrayed in Figure 15. 


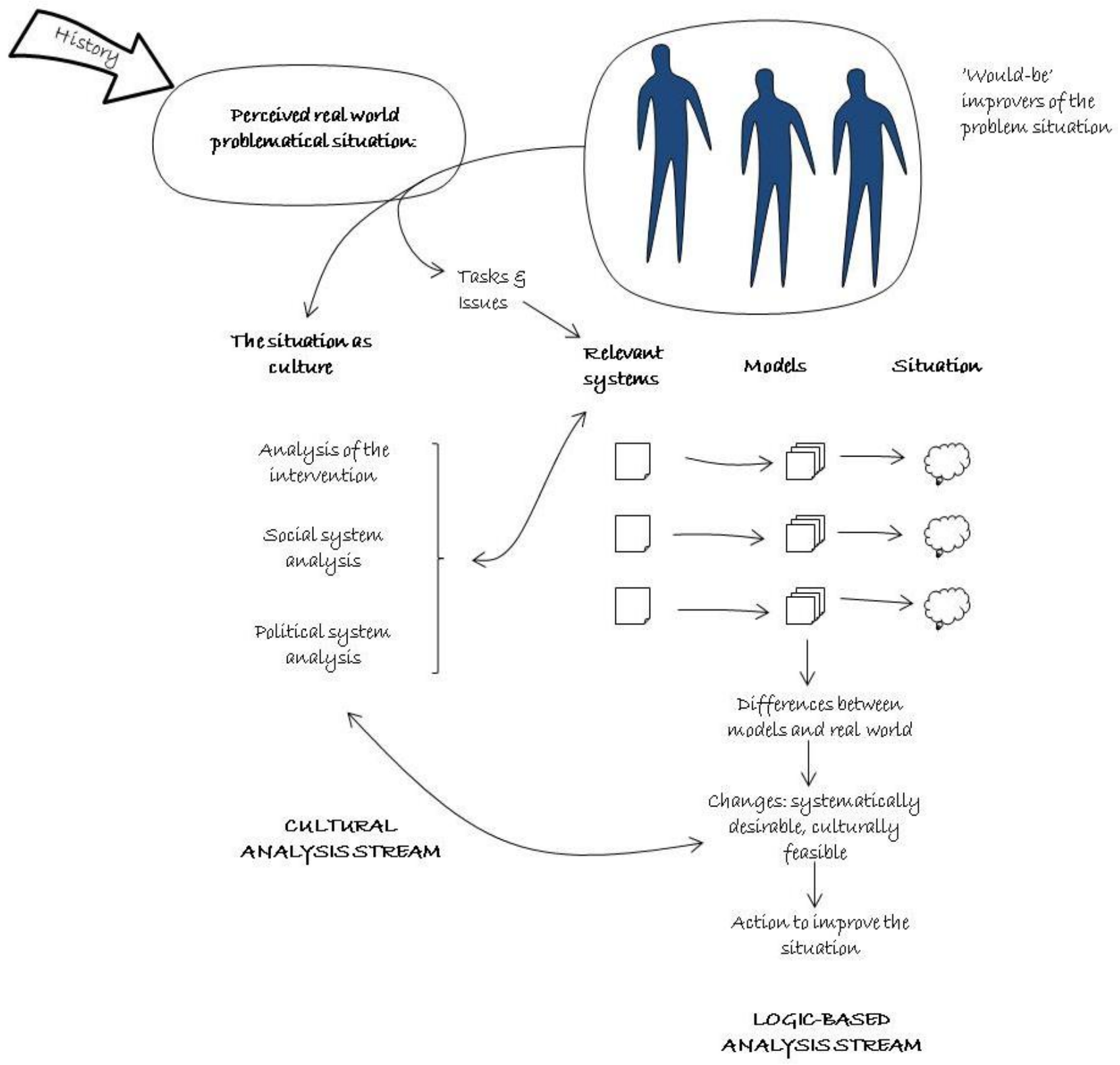

Figure 15 SSM - the methodological process. Adapted from Checkland and Scholes (1990) p.29

These streams of inquiry appear in parallel, however, all elements are interconnected within cyclic processes, so that the two strands merge as one. In a later publication, Checkland and Poulter (2006 p.12) portray the basic process of SSM in a simplified manner, which is seen in Figure 16 incorporating the problematic situation underpinning this research dissertation. 


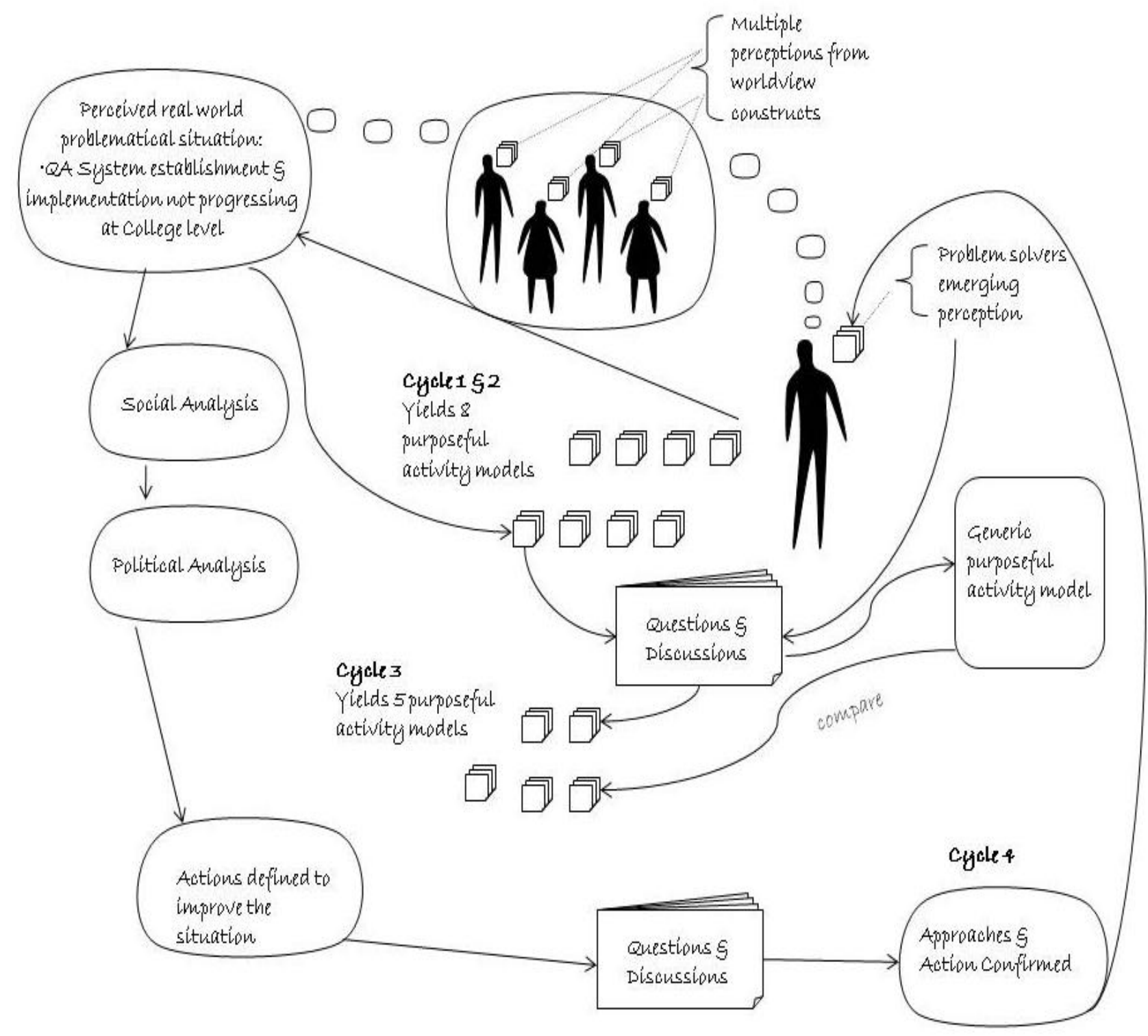

Figure 16 Incorporating current problem situation into SSM's basic process

\subsubsection{Cycle One and Two (SSMp) - The beginnings of the inquiry process}

The perceived problematical situation as described in 3.2 emerged through the academic year of August 2005 - July 2006. These first two cycles of enquiry (Table 5 in section 4.2) commenced more informally in my role as QA co-ordinator, as new systems were being implemented. Challenges being faced seemed to indicate (from my perception) that the problematical situation originated from inadequate knowledge management systems in association with immature organisational capacity planning, both of which were impacting on the effectiveness of the organisation. In talking with other stakeholders both informally and in management meetings to 'find out' what others perceived 
about the situation, it became apparent however, that the problematical situation was more complex and unclear. Factors that arose in this initial finding out seemed to warrant both descriptive and more normative social analyses, with the principles and actions of SSM catering for this form of inquiry. A couple of cycles of action had therefore taken place on receipt of College approval (September 2006) prior to the actual 'formal' exploration into the 'ill-defined' problematic situation, which began subsequent to RMIT ethics approval in December 2006.

As suggested by Checkland and Winter (2006), when using SSM as an interventionist approach, there are in principle two uses when enquiring into a human problematical situation. One use is in relation to addressing the content of the problematic situation, ' $\operatorname{SSM}(c)$ ' and the other is using SSM in planning the approach and the intellectual process of the intervention 'SSM ( $p$ )' (Checkland and Winter 2006). The 'Finding Out' is not an 'initial step' as would be seen in other research methodologies, rather it is part of an ongoing process of inquiry that evolves through the learning cycles of action. The research in this dissertation may be seen to have involved at least two cycles (SSMp) which involved planning the approach prior to including outcomes from the total number of formal interviews which were not completed until end of December 2006 - January 2007. My role as researcher 'practitioner' utilised these first couple of cycles as an 'intellectual process' to ascertain how best to proceed with the intervention based on other perceptions of the situation, what activities were possible, what actions were feasible and reflections on what was and wasn't working.

\subsubsection{Cycle One and Two - The Finding Out through making 'Rich Pictures'}

This initial 'finding out' about the problematical situation benefited from drawing 'rich pictures' to incorporate the apparent multiple factors and issues in the situation. Information and data were collected through making notes of my own perceptions and keeping a record of formal and informal interactions with stakeholders in different roles and situations. The 'rich picture' therefore commenced as a subjective representation of the problematical world as perceived by myself as researcher, then later evolved to incorporate my interpretations of other 'issue or ' ${ }^{2}$ roblem owner' constructs together with their perceptions as described by themselves.

Developing 'rich pictures' of the situation was influenced by identifying stakeholders, exploring 'problem owners' perceptions, reflecting on elements arising from different actions of analysis, reflecting on elements in the first two cycles of inquiry (SSMp), making 'purposeful activity models', comparing these with real-world perceptions and taking action, with the cycle or interactive process continuing. Details are presented in Chapter 4.

\footnotetext{
${ }^{2}$ See glossary of terms
} 
The initial 'rich picture' development commencing with SSMp, helped frame the research proposal and ethics application developed and submitted for approval to RMIT in September 2006. Permission was granted by the College Director to pursue the project investigation within the higher educational institute at this time, so initial interviews and data collection for the project commenced. Limited applied research had been undertaken by other employees within individual colleges at the time of project initiation, with this being reflected in there being no formal institutional research application or ethics approval system in existence. Sign off was the remit of the Director, which at a later stage most likely contributed to the conflicts that arose, due to the centralised Arabic administration finding out by default about the research being pursued. The apparent misinterpretation by the Institute's Central Services administration about the aims of this Doctoral thesis was possibly influenced by socio-cultural and political dimensions of the context, with the consequences and outcomes being explored and discussed in Chapter 5 .

\subsubsection{Cycle Three (SSMc)}

\subsection{The finding out through rich pictures}

Development of the 'Rich Pictures' continued through the third action cycle to address the content of the problem situation, with 'problem owner' perceptions from more formal interviews being incorporated into the evolving emergent activity models created in SSMp. Permission needed to be received by RMIT before 'owners' of the issues in the problematic situation could be identified and approached with the research proposal. However a number of meetings and discussions had taken place as part of the College approved inquiry. Once RMIT approval was gained, fifteen members of the Institute's management team were interviewed over a period of a couple of months between October 2006 and January 2007. More than 23 managers or administrators comprised the College's management team and all were invited to participate. Time was allocated by the Director at a number of management meetings for this research project, with lay statements, consent forms and background information being tabled prior to project implementation. A number of managers declined to be involved, others did not respond to any communication and others initially agreed, then changed their minds and withdrew.

The 'finding out' in the formal interviews involved exploring individual perceptions on organisational effectiveness and the interconnect with QA. The interview activity aimed to be 'open' and nonprescriptive, aiming to encourage 'problem owners' to express their perceptions of the problematical situation. Therefore three very general questions were used to prompt or initiate the discussions:

i. What factors do you consider are critical to the effectiveness of an organisation?

ii. How do you see Quality Assurance systems correlating with organisational effectiveness? 
iii. Giving consideration to both of these questions, where do you see the college we are working in 'fitting' in relation to effectiveness and quality systems?

Data was recorded using hand-written notes, interlinked lines/arrows to connect factors and diagrams to portray what was said. These notes were 'tidied' up in a readable fashion and returned to each individual interviewed to confirm if their perceptions of the situation had been recorded accurately or if there was anything noted that they felt misrepresented their opinion. Subsequent changes were made (if necessary) and these were confirmed again with those interviewed. Singular 'rich pictures' were drawn to reflect perceptions from each interview, as well as another picture which was compiled gradually through reflections after the interviews. This overall 'rich picture' (see Figure 34) emerged and evolved through the total inquiry process, never really being finished, with the flux in relationships and changes in focus being reflected as snapshots in time according to perceptions of the situation at hand. Any 'new' or emergent ideas that were indirectly mentioned or raised formally in conversations with those concerned, were incorporated. The benefits in creating and using 'rich pictures' “...to capture, informally, the main entities, structures and viewpoints in the situation, the processes going on, the current recognized issues and any potential ones" (Checkland and Poulter 2006 p.25), included a more accurate representation of the complex nature of social relationships existing within the context.

\subsection{The Finding Out - focussing on the intervention itself (Analysis One)}

Further development of the initial rich picture resulted from the first Analysis in the SSM cultural stream of inquiry. This involved reflection upon those who held 'key roles' in the problematical situation. These three key roles encompassed - the 'client', those who had caused the intervention to happen, the 'owners', the people who are affected or concerned about the situation, and the 'practitioner' or 'problem solver', who is the researcher or person conducting the investigation (Checkland and Poulter 2006). In the research described by this dissertation, the 'client' was seen to be associated with the Arabic system of governance comprising Chancellor, Vice Chancellor and Arabic Board of governors. Although the College Directors all were involved in discussions about the system's accreditation process, the decision to move forward with a western derived accreditation and quality assurance system was the remit of those holding ultimate power. As researcher in this dissertation, I was the 'participant' or 'problem owner', although I also held several other roles, including Departmental Chair, QA co-ordinator and project manager. Therefore my role as Doctoral student and practitioner carrying out the study as 'purposeful activity' involved another role duality from also being a member of the management team and responsible for an academic department. 
Factors associated with this embedded role are discussed in 3.7 in relation to issues of rigor, and although SSM assumes the researcher is 'in-context', the interrelationships and potentially conflicting interests need to be considered.

All employees were directly or indirectly affected by the new QA system, so in essence all could be seen as 'owners' of the problem or related issues. However, given the responsibilities departmental managers held in relation to QA implementation, it was these individuals who were identified as the 'owners' for purposes of this study. They are thus referred to as 'problem owners' in the data presentation and discussion sections in this research dissertation. Analysis One of the 'finding out' therefore involved reflecting on these three different 'roles' and making notes about perceived 'agendas' behind the intervention. Elements emerging from the exploration of the worldviews of the 'owners' of the problematical situation were then incorporated into rich pictures and summarised thematic records, to contribute to the understanding and perception of the situation.

\subsection{The Finding Out - through Cultural Analysis (Analysis Two)}

Analysis Two (the social analysis) and Analysis Three (the political analysis) were also implemented concurrently. Although Checkland and Poulter (2006) make reference to analysis two as questioning the 'culture' that exists, an enactivist view assumes the nature of cognition is inseparable from context, with social, political and interventional factors being intertwined determinants of the situational 'culture'. One of the particular challenges for this research was to portray or represent what the 'culture' was. The socio-cultural heterogeneous nature of the expatriate 'problem owners' created a mosaic of worldviews, so that determining what action was 'culturally feasible' became one of the main hurdles. In creating models representing the social make-up of the situation, the roles, norms and values of individual problem owners were explored. However, the political analysis in Analysis Three was integral to identifying influences that affected behaviour. Such influences apparently resulting in owner behaviour involving 'informal roles' which at times seemed in conflict to values implied or expressed. That is, the roles within the organisation had associated norms of behaviour which reflected politically derived values originating from the Arabic system of governance. These issues will be presented in more detail in Chapter 4, with discussion as relevant to the aims of this research being pursued in Chapter 5 . Consideration of socio-cultural issues actually preceded any formal interviews.

Perceptions were incorporated in the initial rich picture development prior to official commencement of the project. These were then further developed in Analysis Two, through a process of ongoing reflection and note taking subsequent to the numerous interactions that took place both internally and externally to the work environment. Trying to unravel 'what was going on' 
to understand the dynamics of the situation involved perceptions that evolved through interconnecting with others and their lifeworlds. This process began around August 2005, contributing to the focus and application of the research dissertation, and continued for a number of months even after my employment with the organisation ceased at the end of December 2006.

\subsection{The Finding Out - through Political Analysis (Analysis Three)}

As noted by Checkland and Poulter (2006) and mentioned briefly under Analysis Two, the political dimension of a context will influence the cultural feasibility of action as interventions. The perceived political issues were not raised in the larger forum of management meetings, but the consistency in which 'problem owners' raised the power of politics and the tribal nature of behaviour permeating the organisation was notable. Thus, in reflecting on informal and formal discussions, the presence of powerful vested interests was notably a force that needed to be accommodated for due to its potential destructive nature as perceived by the 'problem owners' of the situation. Again, data and record of situations, formal and informal were kept throughout the research process, with reflections on interactions and outcomes of interventions noted. Elements of this analysis, as for the Analysis One and Two, were incorporated into the overall rich pictures, but sensitivity to portrayal of power relationships, particularly in relation to tribal behaviour, influenced the detail planned for public viewing.

\subsection{Making purposeful activity models}

'Purposeful activity models' are based on the assumption that in all situations people act in a purposeful way for a variety of reasons unique to each participant (Checkland and Poulter 2006). These models should be seen as concepts or 'intellectual constructs' as representing 'ideal types' and represent a subjective view as expressed from an individual's life world (Checkland and Poulter

2006). Pictorial representations of the models of purposeful activity helped to work through the complexity of the situation in a structured manner as forming the basis for inquiry. Each individual perspective incorporated into the research process, represents a description of the real world, i.e. they model "...one way of looking at complex reality..." (Checkland and Poulter 2006 p.38).

The Logic-Based stream of enquiry or descriptive research strand therefore involved comparing the worldviews of stakeholders about particular issues and factors, as drawn from interviews, meetings and discussions, to more specifically ascertain a variety of perceptions of the problem situation and the 'models of purposeful activity' described as individual realities of the situation. These models were then compared with 'Root definitions' which were developed through considering the 'what?', 
'how?' and 'why?', which in SSM is referred to as the PQR formula, where the ' $Q$ ' or 'how' reflect the transformational process(es) necessary to achieve desired improvement. The transformational process in actuality defines the purposeful activity, containing elements represented by the mnemonic CATWOE where:

$\mathrm{C}=\quad$ Customers affected by $\mathrm{T}$ as victims or beneficiaries

A $=\quad$ Actors who would do the activities which make up T

$\mathrm{T}=\quad$ Transforming process (based on $\mathrm{W}$ )

$\mathrm{W}=$ Worldview

$0=\quad=\quad$ Owners who could stop (or *hinder) the T process

$\mathrm{E}=\quad$ Environmental constraints which are taken as given in doing (or *hindering) $\mathrm{T}$

(* Italics authors addition based on data collection and analysis)

A primary Root Definition and generic model of activity were formulated after reflecting upon models of purposeful activity as perceived by me as 'problem solver', through working through Analysis 2 and 3 to define a Transformation process. Further details about the elements in the process are presented and discussed in Chapter 4, but for purposes of clarification, the generic Root Definition formulated was:

'An Emirati owned educational system staffed by a diverse cultural mix of expatriates and nationals who work together to deliver tertiary educational programs at credible international standards, incorporating ongoing evaluation and review of business practice to improve effectiveness to ensure organizational competitiveness and sustainability'.

In discussions both with individuals, in small groups and in management meetings when QA implementation as a model of purposeful activity was on the agenda, the desired Transformation process required a number of agreed criteria to determine if improvement in relation to expected outcomes ( $E_{1}$ efficacy), utilising available resources ( $E_{2}$ efficiency) or longer term change $\left(E_{3}\right.$ effectiveness), were being met. Whilst efficiency was reasonably well defined, the perceived lack of resources was one bane of contention, conflicting perceptions on expected outcomes were another, whilst agreement on what comprises 'effectiveness' and appreciating the value in envisaging 'longterm' change were additional challenges encountered.

In all, five models of purposeful activity were developed to structure discussions, all being seen to share a defined 'primary task system' stated as: 'the College is a system that provides tertiary education to national Emirati women to a standard aimed at benchmarking against 'western' best- 
practice education'. The 'issue-based system' related to the different transformations represented by the five main activity models as described in Chapter 4 .

\subsection{Using the models for questioning and discussions}

Thus through analysis and reflection upon perceptions and defined realities of individuals and groups involved in this research project, five models of purposeful activity were developed and compared with the generic model to facilitate the evolution of a more effective organisation and move the QA process forward. These models were not designed as the 'answers' to any perceived problem, rather were aimed at initiating and structuring further discussion on the way forward. "...purposeful activity models simply enable our organized discussion to take place" (Checkland and Poulter 2006 p.50).

Whilst questioning the situation would have been most beneficial at management meetings between July 2006 and December 2006, some major organisational restructuring saw a new College Director appointed. Despite this Director also agreeing to the project continuation, there was limited opportunity to move through the enquiry process at formalised meetings due to new and different Directorial priorities. Furthermore, whilst the previous College Director had given approval for the project, a number of management team members subsequently indicated they did not want to be involved, despite potential benefits for the whole organisation. Therefore a more formal chart matrix of activities, themes and relationships was compiled to form the basis of small group and individual discussions (Table 8), given that the opportunity to present the evolving rich picture descriptions to the management team as a whole was now restricted.

\subsection{Defining - as opposed to taking - action}

The aim of the discussions was 'to find an accommodation' amongst the culturally pluralistic management and ultimately, faculty teams. As noted in previous sections of this dissertation, true consensus is an elusive concept given the unique nature of emergent cognition. SSM ably assisted in creating these 'accommodations' to enable the defining of actions to improve, as opposed to attempting to seek 'consensus'. The presence of a diversity in world views, together with a complex socio-political environment created a challenging context in which to initiate a social inquiry process to bring about improvement in a problematical situation. Through individual and small group meetings a number of recommendations were made to move forward (i.e. defining the action). Yet, whilst a number of structural, IT and administrative systems approaches saw the beginnings of minor changes, implementation of the research project was cut short with the defining of actions near the end of Cycle 3 prior to significant changes in processes and behaviours were evidenced. 


\subsection{Reflecting upon the situation - rather than action (SSMc - SSMp)}

Enabling action was necessary due to the complexity in context and social nature of the project context. Although at least two preliminary action cycles (SSMp) of learning preceded the formal interviews (SSMC), the more comprehensive actions planned only reached the definition stage, rather than moving through a whole new 4th learning content cycle. Limited opportunity to implement the recommendations was due to the researcher's ('problem solver's') contract being terminated suddenly in December 2006 at the point of action implementation. As noted by Checkland and Poulter (2006) "Some studies will be ended after defining the action, some after implementing it" (p. 14). This study, for reasons which will be discussed in more detail in Chapter 5 , did not proceed to implementing the defined actions, however further small group meetings, and communication via email continued outside the workplace for another six months as a 4th cycle of process action (SSMp), with further conscious critical reflections and analysis contributing to research conclusions.

\subsection{The issues of rigour}

The concept of rigor in relation to qualitative research does not need to assume data is quantifiable, generalisable and measurable as is the case in quantitative research. Rather the terminology is associated with setting a particularly standard, which in qualitative research may be benchmarked against the 'measures' of reliability, validity and ultimately, defensibility. As noted by Neuman (2006) reliability and validity as concepts in social research help "... establish the truthfulness, credibility or believability of findings" (p.188). The relational notion of reliability and validity usually sees these two research elements as complementary, yet at times may conflict according to the constructs involved (Babbie 1998). With reliability being associated more with 'dependability' or 'consistency' and validity more closely aligned with being 'truthful' or 'authentic' (Neuman 2006), perhaps it is the constructs underpinning validity which need to be emphasised in qualitative research.

The focus of qualitative research involves exploration of the human condition in socially complex contexts, with the inherent dynamism warranting approaches that are flexible and non-static, yet consistent and authentic (Neuman 2006). Coghlan (2007a) also suggests 'authenticity' as being “... at the heart of being human, as being human means to be experiencing, understanding, judging and deciding" (p.338a). He draws upon Lonergan's (1972) philosophy on the structure of knowing, to suggest that praxis-reflection methodology as underpinning action research necessitates an authenticity which can be assured by: being open to experience (being attentive); asking questions, seeking understanding (being intelligent); critically questioning, being curious, promoting discussion, 
allowing dissent (being reasonable); choose what we think we ought to do (being responsible) (р.338).

It may be seen that associating reliability and validity with measures of certainty and predictability fits better in a positivist framework, as qualitative research particularly when employing image-based descriptive data is "...better served by questions about power and influence, adequacy and efficacy, suitability and accountability" (Watling 1995 p.5). Authenticity, therefore, as a legitimate 'measure' of research quality complements Lincoln and Guba's (1981) value of 'trustworthiness' in relation to naturalistic inquiry involving qualitative approaches to the study of groups of people in their 'natural setting. Lincoln and Guba's criteria of credibility, transferability, dependability and confirmability are designed to reflect interpretative and exploratory approaches to enquiry into the nature of social contexts, as is relevant to the research described in this dissertation. These criteria have in turn been expanded upon by numerous authors to include other elements constituting quality and rigour in qualitative research. For example, Simco and Warin's (1997) criteria of completeness, adequacy of interpretation, transparency, self-reflection and the aggregation of conflicting interpretations; Cicmil's (2006) focus on credibility, trustworthiness and relevance, involving an 'objectivity' in interpretative approaches to encompass honesty, ethics and high moral standards; Neuman's (2006) measures of validity based on plausibility of statements, diversity and dense connectivity of empirical data; and the 'recoverability' of the research facilitated through open declaration as to the language or intellectual framework such as is found in SSM (Checkland and Scholes 1990).

Authenticity thus demands a degree of integrity in different forms of qualitative research, but in particular, is pivotal to action methodological approaches where the embedded nature of 'researcher-in-context' is integral to the enquiry (Checkland and Poulter 2006; Coghlan 2007a). Systems thinking within an enactivist paradigm, privileges the view of emergent perceptions and understandings. Authenticity and truthfulness originates with the self, for potential transformation of self as opposed to transformation of a context. As noted by Floyd (2008), the knowledge which is generated or emergent is emancipatory and “...involves one's ability to critically reflect upon and examine established commitments, assumptions and biases" (p.143). SSM draws upon mental models of 'others' who are embedded in context inextricably linked to the situation which is identified as problematical, with their perceptions defining the problem as the first step towards finding a solution (Checkland 1981). The researcher-in-context uses SSM as a process to facilitate debate to reach a 'shared' accommodation in understanding about a problem and action necessary, drawing upon Habermasian and Schutzian assumptions of an intersubjective world. Validity of knowledge is dependent upon what stakeholders perceive 'as real', with the methodology of SSM facilitating an 'insider's' contribution to knowledge development through participation in a world as constructed through interconnected domains of subjectivity, intersubjectivity, objectivity and 
interobjectivity (Maturana 1988; Habermas 1992; Floyd 2008). Criteria as relevant to this concept of knowledge validity are drawn from Habermas's claims of 'truth, sincerity and justness', with SSM as a systems methodology being seen to unravel some of the complexities found in human problems (Checkland and Scholes 1990; Floyd 2008).

SSM as underpinned by action research theory, can facilitate authentic enquiry through incorporating Reason and Torbert's (2001) 'three voices' of action research. First person inquiry involves individual reflection into own actions and the world around them. Second person inquiry takes place when an individual through dialogue communicates with others about an issue of mutual concern. And third person inquiry is an extension from first and second person inquiry, where learning is informed from the first two means of inquiry, from which decisions about what is actionable takes place. According to Coghlan (2007b), a researcher will demonstrate the integrity that a research doctorate requires through integrating these three forms of inquiry into the research methodology. As described in 3.6.2, the process of enquiry in SSM may be seen to involve such 'three voices' of action in an ongoing cyclic, looking-forwards, looking-back reflective manner, with the researcher-in-context holding an 'insider' role. This together with the criterion of 'recoverability' combine to move beyond the measure of 'plausability' (Checkland and Holwell 1998), with it being suggested that the utilisation of SSM provides the structure to recover the enquiry process, despite changes in context and stakeholder involvement. Such recoverability as assuring validity however, does not necessarily mean that the identification and perceptions of a problematical situation will have any semblance of similarity, as 'repeatability' is not a measure of qualitative research, particularly given the uniqueness of human mental models as individually emergent. Rather, 'truthfulness' of the enquiry involves pragmatic exploration and description into the historical, cultural, social and psychological aspects of the context to critically understand the multiple perspectives of the problematical situation (Yu, Moon et al. 2008). Openness to evolving and shifting research project goals as the problem situation unfolds is necessary to avoid 'setting an agenda' too early on in the problem structuring approach. Ongoing goal interpretation and re-interpretation assists in emergent knowledge, counteracting some of the criticisms of SSM if goal identification is rigidly applied at the project onset without allowing for changing circumstances (Pollack 2007).

Dual or multiple researcher roles can raise issues of loyalties (organisational and academic), potential bias and a subjectiveness in interpretation of data. Such potential conflicts can derive from situational 'pre-understandings', 'role dualities' and involvement in 'organizational politics' (Coghlan 2007a). These 'dual loyalties' need to be declared and addressed in the research to avoid the 'orthodox academic majority' being supported in their perceptions of action research as being nonscientific (Calori 2002). Pre-understandings need to be explored and articulated, role duality needs identification and acknowledgement, and awareness of organisational politics necessitates an 
astuteness and sensitivity to appropriateness of actions (Coghlan 2007a). SSM may be seen as a methodology which facilitates social enquiry into human problematic situations that entail a “...blooming and buzzing confusion and complexity..." (Checkland and Winter 2006 p.1436). As researcher, I held multiple roles in the organisational context - both as project manager, departmental manager, doctoral research student and College management team member, which all added to the contextual mayhem. However, the rigorous nature of SSM as described, not only accommodated for the embedded nature of researcher-in-context through taking "...seriously the subjectivity which is the crucial characteristic of human affairs and to 'treat this subjectivity, if not exactly scientifically, at least in a way characterized by intellectual rigour'" (Bergvall-Kåreborn 2002 p. 28 quoting Checkland 1981 p.30); but SSM also provided for exploration into organisational and social politics and a rigorous reflective process into positioning of self.

Quality should therefore be seen as dependent upon the appropriateness of the research design, the nature of rigor and 'truthfulness' in the process of inquiry and the relevance or usefulness the research may have to relevant professional and academic communities (Agostinho 2004). Transparency in process, together with ongoing confirmation about data validity from 'problem owners' as identified in the research, were pivotal to this enquiry. Information was returned to those interviewed to ensure accuracy in recording of perceptions, with these then being compared with notes made, formal reflections, accurate diary logs and minutes of meetings. All these actions contributed to addressing the issues of authenticity, attentiveness and responsibility with the utilisation of SSM as a structured approach to the process of social inquiry also facilitating the truthfulness and recoverability of the project. Martinsuo (2001) has suggested that qualitative research can tend to be too descriptive and lack analytical rigor. However, it is felt that through using SSM within a defined conceptual framework to clarify beliefs about knowledge and its creation, that the validity and quality of the findings can be promoted. SSM has been criticised as failing to adequately address the powerful interplays that exist in complex human situations, where possible tensions between disparate groups of individuals, potential conflicts between researcher and researched, together with other power plays of vested interests, systems of governance and so forth all take place (Flood in Floyd 2008). However, through transparently acknowledging the researcher role and possible biases, and through logically analysing the political and social elements of the context as perceived by self and others, it is felt that these potential shortfalls can be accommodated for.

The research described in this dissertation is in partial fulfilment of the Doctorate of Project Management. Pettigrew (2001a) has suggested that quality and quantity of management research from doctoral programs in various fields of management has been in question due to a tendency to “...disengage from related theoretical and empirical development in social science disciplines such as 
economics, sociology, psychology, anthropology and political science" (p.S64). This research dissertation has made an attempt to incorporate sociological theory into the framework to address such issues in question, drawing upon enactivism as a cognitive theory to incorporate a pragmatic epistemology and becoming ontology as supported by SSM in a process of social enquiry. Actionable knowledge from iterative cycles as evolving and in constant comparison with, and through the process of cultural analysis stream, not only supports the emergent nature of knowledge useful for both professional and research communities (Coghlan 2007b), but add to the rigorous nature of the research approach. The actionable nature of emergent knowledge and the integrative approach that SSM supports through first, second and third person inquiry add to the integrity and ultimately the quality of the research outcomes.

According to Sankaran, Hou et al (2009) SSM provides opportunity for theory development which action research in general is missing, due to SSM's rigorous methodological approach accommodating for enquiry into the imprecise, dynamic, non-linear nature of human affairs. SSM has been portrayed as a relatively mature and established methodology (Checkland and Winter 2006; Dodouras and James 2007), moving beyond other subjectivist approaches influenced by soft systems thinking, to enable respondents as stakeholders to articulate the construction of their individual social realities (Bergvall-Kåreborn 2002). Modernist research has tended to perpetuate the separation of theory from practice (Pettigrew 2001a) and fails to accommodate for an assumed continual creation of social realities. Consequently a conscious decision was taken to incorporate postmodern thinking in the research approach, together with applying enactivist theory and SSM to close this theory-practice gap to better address the social complexity in the problem situation. Such an approach demonstrates a "...greater sensitivity towards practical complexity [and] will prompt a more comprehensive notion of rigour" (Whittington, Pettigrew et al. 2001 p.486), with the heuristic methodological approach of SSM providing a structured set of guidelines which supports the validity and reliability of results generated (Mingers 2001; Schwaninger 2006). It is concluded that ensuring transparency in conceptual assumptions and enquiry processes together with disciplined thinking and an openness to different ways of thinking, facilitated the scientific rigor required for validity in the research process (Schwaninger 2006).

\subsection{Ethical considerations}

As noted by Cicmil (2006) "...ethical and moral considerations are seen as part of action in complex project environments" (p.33). Transformational change projects involve a social complexity which is dynamic and unpredictable, involving messy human situations where conflicts and power struggles result from disparate understandings (Cooke-Davies, Cicmil et al. 2007; Engeström, Kerosuo et al. 2007b). Tensions that result from such real-world problems will inevitably produce ethical dilemmas 
for the social researcher and even more so for the researcher embedded in context. Ethical standards and the morality of research intertwine, with the researcher being the one needing to determine the costs and benefits of the social enquiry process (Neuman 2006). SSM as a process of social enquiry will inevitably involve moral and ethical issues due to exploring and comparing individual perceptions of a social situation, and ultimately deciding on when and how to take action (Yu, Moon et al. 2008). Moral reasoning and research integrity is dependent upon "...the understanding and formation of the participants-driven approach to build ethics for critical systems practice" (Yu, Moon et al. 2008 p.293). SSM assumes that purposeful action is a constant and that individuals differ in meaningful attribution given to a particular event or situation. SSM not only has the potential to 'map the terrain of thinking about a situation' but can also 'reveal the nature of relations among people' within a specific contextual setting (Córdoba and Farquharson 2008). Ethical research is therefore dependent upon what the researcher thinks 'ought' to be done, the values which drive the enquiry and the overall integrity of research and process (Neuman 2006).

The research described in this dissertation has used SSM as the transformative approach for the enquiry, moving beyond constructivist theory through incorporating enactivism as the critical approach to interpretation. Ethical issues and values in such a research conceptual framework involve the inclusion of formative values and intrinsic ethics, where a 'moral tilt toward revelation' takes precedence over a 'process tilt' (Guba and Lincoln 1994). As noted in Section 3.5.3, the purpose of the inquiry involved a combined exploratory and descriptive approach to not only understand and reconstruct a problematical situation, but to critique and transform the social context through direct engagement. Noting the intrinsic nature of ethics in such an enquiry process, there is a demand for the researcher to be 'revelatory' particularly in relation to consent being informed (i.e. moral tilt) and given the interconnected 'research - researched' relationship, the issues of confidentiality and anonymity are paramount, together with exposing the researcher's intent to protect against deception (i.e. process tilt) (Guba and Lincoln 1994). Guba and Lincoln (1994) also note the importance of values in both critical theory and constructivist paradigms as being "...ineluctable in shaping inquiry outcomes" (p.114). This issue when considered with the interaction between action and reflection in SSM, demands the 'researcher-in-context' appreciates their position of responsibility as involving dynamic understanding. As suggested by Calori (2002), such a relationship however, particularly if acknowledged and transparent, will contribute to the quality of research theory through contributing to knowledge of relationships, context and processes. This has the potential to add to the understanding of moral motives, emotions and feelings of actors, and provide insight into stakeholder agendas and political dynamics (Calori 2002). Research ethics have been identified as involving five main principles - protection from harm, right to privacy, avoidance of deception, informed consent and debriefing (Vaughan and Hogg 2005). 
However, the balance between the subjective nature of a researcher's interpretation and perceptions, and the models constructed through other individual and shared realities should be seen to lie at the centre of ethical consideration in qualitative research. This project has incorporated a critical approach to enquiry and although ethical issues need to be considered as in any research, many of the "... ethical debates, codes of ethics and review boards [may be seen as] inadequate and rooted in positivist assumptions about individual rationality" (Neuman 2006 p.146). Enactivism assumes an embedded emergent nature of cognition bringing with this thinking, a more humanist and ecological approach to ethical thinking. The complex and patterned nature of human existence demands an 'expanded pragmatic' approach to ethical consideration involving self, other and the environment; where "...those who are to be at the receiving end of a decision should be party to the decision-making process or at least very well represented ..." (McIntyre-Mills 2008 p.194). An ethics of emergence is supported by non-linear patterns of cognitive transformation, where temporality and norms of action facilitate adaptive response to move beyond the circularity of closed systems as we move into the future (Bausch 2008a).

Noting the issues of quality and validity in research, it is suggested that in qualitative research where the complexity of human relations dominate the most obvious ethical issues emerging from context, will be those pertaining to power and trust and how these interface with dialogue. Given the nature of the research described in this dissertation, the relationship between me as researcher and other management team members interviewed needs to be acknowledged. These relationships were based on collegiality due to functionally assuming the same role level of departmental managers. However, the making of notes from more informal encounters with faculty and assuming the position of Quality Assurance co-ordinator, inevitably brought with them issues of trust and avoidance of abuse of power, which was addressed primarily through transparent communication and open progress reporting at formal monthly management meetings and at more frequent informal meetings.

In relation to processes followed to address real and potential ethical issues, such transparency in approach was paramount. Permission was sought and given from the College directorship and presentation of the proposed project was made at a senior management meeting. Whilst it was obvious that the Director had approved the project, there was no obvious persuasive support, other than providing time on the meeting agenda. This actually was a disadvantage in some ways, as gaining buy-in from stakeholders was one of the challenges faced as the project progressed, and having directorship 'drive' may have assisted.

The Arabic Central administration was not directly or formally informed by me as to the research project, primarily due to the degree of autonomy of individual colleges but also the lack of any centralised research process subsequently meant there was no imperative to do so. The Director 
however said the proposed research was informally raised with the Vice Chancellor (VC) but other than a request to not identify the organisation by name, no further action was required. It was foreseen by me as researcher, that outcomes of the research process may be beneficial and transferable to other colleges. A different centralised approach however, to informing relevant stakeholders about the research would need to have ensued if the scope was to extend beyond the one College.

The usual Ethics approval for research involving human participants was sought from the RMIT Human research Ethics Committee and permission granted in December 2006 after 'sample questions' and a description of 'rich pictures' were forwarded to the committee. This in itself was unusual given the qualitative and exploratory nature of the research. Requiring and assuming that there would be 'set' questions and the unfamiliarity with SSM as a research approach, appears to be reflective of a persistent positivist research paradigm that dominates intellectual and academic inquiry. Broad indicative thematic questions were submitted, but the themes or research aims should have sufficed in the ethics approval process. The College had given approval for an exploratory project into QA implementation in September 2006, thus a number of interviews to move this forward commenced, but data not fully incorporated until RMIT approval and signed consent forms received in December 2006.

Informed consent was sought from management team members identified as 'owners' of the issue or problem. This was subsequent to provision of a lay statement explaining the research aims and process and brief discussion at a management meeting. A number of participants returned their consent forms signed within the next couple of days, whilst others did so in response to follow-up emails and face-to-face communication. A number of team members did not return the consent forms and did not respond to email communication, so I assumed they had chosen to not participate and this was respected. This did create challenges further into the research process, as presenting data for discussion at management meetings became increasingly difficult, as all members had not agreed to participate. Thus smaller group meetings and individual follow-up became necessary.

None of the management team members were in a vulnerable position in relation to me as researcher, however, the issue of possible identification of respondent data became the critical concern of most. The fear of disclosure will be discussed further in Chapter 5, but measures to ensure anonymity included a numbering system on interview data and pictures, with identity correlations only being available to myself as researcher and this information being left at home securely stored. Although potential for physical harm was absent, the potential for psychological harm was a real concern given the tenous nature of expatriate employment contracts and the systems of operandi that tended to cease employ suddenly if there were signs of dis-content. One problem owner expressed such fear for her position the day subsequent to her being interviewed, 
that she insisted her consent was to remain verbal, that she would not reply to anything in email and she requested re-confirmation that data could not be traced to individual respondents. There was no undue pressure in relation to consent for involvement, nor deception about the aims or research process being followed. As mentioned, consent was informed and agreement to participate voluntary. The College Director did not play any part in endorsing or encouraging participation, which if present, may have introduced particular issues of power and coercion.

Confidentiality was also assured by data being returned for validation in hard copy only. Email was used for general correspondence, but soft copy interview and meeting data was not circulated in a format that could be forwarded on to others. Anonymity was also supported by the SSM process itself, where pictorial representations of worldview constructs removed any identifying features of individual participants. Statements made by the 'problem owners' also have been compiled as a whole rather than as participant $1,2,3$ etc to reduce the likelihood of identifying individual management team members by their responses. The overall rich pictures of the problematical situation are a compilation of different perspectives, and there was no discussion with participants about who else had been interviewed or was involved.

It should be noted that researcher notes were made on reflection of informal conversations and meetings, both College and system-wide. These notes added to the cultural analysis stream with the emphasis being on researcher perception of interactions in context, rather than on behaviour of specific individuals. Autonomy of these 'others' within the organisation were respected with no identifying notes being made. The impracticality of seeking informed consent for the reflective notes and subjective interpretations from numerous encounters, was balanced with respecting the privacy of individuals.

The focus was on social enquiry into a problematical situation for transformational change. Given the complex nature of the social and political context, all efforts were made to respect the rights of participants to remain anonymous. Confidentiality in data was striven for, together with a transparency in process and a structured research approach to the problem at hand. Ethical thinking was contextualised through enabling a participatory decision-making process involving "...systemic cycles of contextual dialogue and questioning by the stakeholders who are to be affected by decisions" (McIntyre-Mills 2008 p.206). Using SSM to guide the social inquiry process not only aided the exploration into the problematical situation, but provided a framework of iterative cycles of reflection and dialogue to facilitate the emergence of contextual ethical decision making through involvement of all stakeholders. 


\subsection{Chapter Summary}

The research described in this dissertation is guided by a defined philosophical conceptual framework which assumes a pragmatic epistemology and becoming ontology to support the actuality of projects as social process and human activity systems in a state of flux. Soft Systems Methodology (SSM) has been selected as the structured approach to use in the enquiry as its process facilitates connecting normative and descriptive research strands to better explore messy ill-defined problems. The enquiry into the perceived real-world problematical situation in the implementation of a QA system in a middle eastern educational institution, involved four cycles of action learning, two initial cycles involving the planning of the approach and the intellectual process, one working through the content of the problem situation and a fourth which involved further discussion and planning for future endeavours.

The choice of methodology drawn from soft systems thinking has been informed by the reported inadequacies of traditional rational approaches to project management research and practice. 'Future' directions of project research (Winter, Smith et al. 2006) have noted the increasing complexity of the project environment, where cognition as emergent sits outside systems of 'control and predictability' typifying positivist thinking. The social nature of project praxis demands a methodology which can accommodate for discursive socio-cultural elements, where the whole of the project entity entails properties which are greater than the individual parts. An emphasis on the process of enquiry as being the system actuality, moves thinking beyond efforts to measure and define specific outcomes. Enactivism as a theory of cognition supports the emergent yet embodied view of cognition arising from interconnected human elements coupled with an environmental context.

Project practice is now moving beyond traditional professional boundaries with complexity from illdefined situations being better explored through employing interpretative and critical approaches to enquiry. Postmodern thinking supports the dismantling of metanarratives that fail to adequately explain today's ambiguous and ever-changing realities. Cognition as embodied and inseparable from a system where learning emerges through action and reflection is the enactivist view which complements postmodern and critical thinking approaches to social enquiry through identifying a multiplicity of perceived realities in seeking to make improvements to problematical situations. SSM as an action methodology adequately enquires into messy human situations, working within a qualitative framework to incorporate pictorial representations of perceived realities to assist with the 'finding out'. The learning process facilitates transformational change, at individual, organisational and social levels, with projects being perceived as ecological systems better symbolising activities which shape today's business practice. 
Exploratory and descriptive research when incorporated into the research approach assists to focus on the 'what', the 'how' and the 'who' of a problematical situation. SSM moves beyond such enquiry to facilitate action for change and transformation, through acknowledging the centrality of the human element in project practice. As a structured approach SSM seeks to achieve accommodations between discursive human perceptions for desirable and feasible change. The research described in this dissertation has built upon purposeful models of activity representing real-world constructs of reality, to define actions for improvement. Given the embedded nature of researcher in context, transparency in approach and situational descriptions has been instrumental in ensuring authenticity and researcher integrity. This research has employed a rigorous methodology to seek improvement into a problematical situation, with a number of resultant desirable and feasible actions being defined to potentially offer effective solutions in socially complex project environments. 


\section{Chapter 4 The SSM Learning Cycle in Action}

\subsection{The Situation}

\subsubsection{Introduction}

As noted in section 3.2 the research described in this dissertation is based on a Doctoral level research project undertaken in a Higher Educational Institute in the United Arab Emirates (UAE) (being referred to as The Gazelle Institute for purposes of anonymity). It was the implementation of a Quality Assurance (QA) project which presented as problematical, with difficulties faced appearing to warrant exploration to unravel what was a complex and messy situation.

Soft Systems Methodology (SSM) was selected to investigate the problematical situation, as the active process of inquiry has the potential to bring about improvement through engaging with socially derived project complexity (Checkland and Poulter 2006). The research aim was to work towards active transformational practice involving cyclic learning processes drawing upon different world views to reach some semblance of 'consensus' in action as the enabling force. Although true consensus in human affairs is really an elusive concept due to differences in cultural understanding as reflected in discursive discourses (Gadamer 1981; Derrida 1992; Checkland and Poulter 2006), meaningful accommodations may be possible despite powerful interplays existing in disparate social groups (Foucault 1982; Edkins 1999). SSM acknowledges these differences and works towards defining action to improve upon a situation identified as problematical. This methodology moves beyond the Habermasian concept of 'communicative action' which is based on ideal situations involving "...intersubjective mutuality, trust and shared knowledge" (Sikka 2008 p.228) to dealing with cognitively 'fuzzy' situations where the emphasis is on exploration of a problem from the multiple perspectives of different lifeworlds to promote unified action that is 'desirable and feasible' by all players concerned (Checkland and Winter 2006).

Within the project organization, complexity arising from disparities in stakeholder social backgrounds became increasingly apparent as a significant factor affecting project process. As noted by Bausch (2008b ) system scientists have been devising "...ways around the problems which bedevil unstructured democracy in complex situations in which people hold divergent views ..." (p.278) for decades. SSM as a never-ending learning cycle and as a methodological approach to facilitating change through reflective action supports the actuality of projects as ontologically 'becoming' and epistemologically 'pragmatic' emergent processes to deal with some of this complexity. Organizational resilience is dependent upon adaptive responses and co-creation, and SSM facilitates 
engagement with complexity to enable the emergent cognition that is deemed necessary for this process.

\subsubsection{The Context}

\section{The country}

This research project was implemented in the United Arab Emirates (UAE), a 'middle-eastern young' country existing in its present political form for only 37 years. The UAE lies south east of the Arabian Peninsula, on the Arabian Gulf, with bordering countries being Oman to the east and Saudi Arabia to the south/south west. Prior to 1971 the UAE was known as the Trucial States or Trucial Oman, comprising independent states ruled by different Sheikhs. The latter half of the 1960's saw the withdrawal of Britain from the region and political independence being established within a number of countries, one of which was the UAE. The dramatic changes that have taken place politically and socially since the discovery of huge oil and gas reserves and the establishment of the UAE as a federated state in 1971 comprising 7 Emirates, has been notably rapid. Nevertheless, the true cultural essence of the Arabic ways of knowing and doing remain strong and permeate all social and institutional contexts.

As noted by Heard-Bey (2004):

"If one compares the environment, both materials and socio-political, of the Trucial states in the earlier decades of the last century with that of the United Arab Emirates in the first few years of the 21st millennium, it may seem as though one is comparing two entirely different worlds. Yet the most important strand in the fabric of the UAE, the society of the native population, has itself changed remarkably little. Attitudes, values, behaviour and customs which were formed under quite different circumstances continue to be essential to the family's life; they are equally essential ingredients in the interaction of today's multinational society and the newly created State" (p.2).

Prior to 1965 the UAE was a tribal society with a homogenous culture in terms of similarities in ethnic background, Muslim faith, and agreement on morality, behaviour and law (Heard-Bey 2004). However, with the influx of expatriates sourced primarily to contribute to the rapid infrastructure developments over the last few decades, a new social heterogeneity has emerged comprising an ethnic pluralism and cultural diversity that is at odds with traditional Bedouin life. Advances in technology and communication systems, together with the ease of international travel, have increased the permeability of the country's national borders, with subsequent transformational 
economic, political and institutional changes being financed primarily by estimated oil reserves lying under the sands. The issues resulting from access, availability and creation of all things 'modern', together with the pace of change which is possible, have created socio-political complexities which the Arabic systems of governance grapple with on a daily basis. The UAE, as for many other developing countries experiencing rapid socio-economic growth, struggles to keep pace with necessary national and institutional policy formation, relying on the import of external consultants, contractors and operational systems to facilitate the nation's transformation (Rust 2000). Yet politically, the appointment of Rulers remain tribal in nature, with family and kinship ties determining the collective nature of decision making as guided by the selected or appointed leaders, whilst the leader is expected to be visionary and competent. "Leaders need to manage the tension between expectations of efficiency, improvement and performance on the one hand, and the more traditional paternalistic values on the other" (Kabasakal and Bodur 2002 p.51)

Members of the UAE national population are predominantly Islamic, of Sunni Muslim faith, where "... social and ethical obligations of a Muslim are based on the belief that the Islamic community is a brotherhood where equality in personal worth, regardless of status and wealth prevail" (Gibb in Kabasakal and Bodur 2002 p.44). The Quran is the unifying force underpinning traditional moral behaviours and social practices bound by Islamic law as represented in Shari'a legal and administrative systems (Kabasakal and Bodur 2002). Tribal Sheikhs as rulers, are expected to exercise their authority within the law, and whilst expertise for the UAE's emergent identity may be sourced from overseas, systems of governance and the power of authority remain the remit of the Emirati population. The cultural heterogeneity evidenced in social life remains distinct from the homogeneous nature of both the nation's political and institutional life, with control of the country's future remaining firmly in the hands of the Emirati nationals who are a minority in number, not wealth or power.

According to 2003 reports, demographic statistics as of 1995 (FDI 2008) recorded the UAE population at approximately 2.5 million or which 1.5 million were non-nationals. A more specific break down of ethnic groups indicated that Emirati nationals comprised only $19 \%$ of the total population, with other groups being of Arab and Iranian origin (23\%), South Asian (50\%) and Western and East Asians (8\%). More recently however, the population has now been estimated at closer to 5 million in total, with the proportion of UAE nationals remaining reasonably constant at 20\% (CIA 2008). The economy has been recorded as having a GDP of $\$ 71$ billion, GDP per Capita $\$ 29000$ and GDP composition of Agriculture (3\%), Industry (46\%) and Services (51\%) (UAE Ministry of Planning 2003), with $40 \%$ of the GDP being sourced from current and estimated future oil reserves (CIA 2008). It is unlikely that the dramatic socio-political transformation of the UAE would have taken place without the discovery of these huge oil and gas reserves in the region over 30 years ago. But with the value of crude oil 
resources being estimated in 2001 at Dh69.6bn and non-oil resources at Dh178.7bn (UAE Ministry of Planning 2003), the real challenge for the country today is to ensure that there is sustained economic growth with a reduced dependency on oil revenues in the future.

\section{The project organisation}

All sectors of the UAE have been influenced by the dramatic socio-political changes over the past few decades, of which education is just one. The College in which the Doctoral level research project was conducted, was one of 12 Colleges in a larger educational system being referred to as the Gazelle Institute for reasons of anonymity. The institute system and the individual colleges were bureaucratically structured with administrative systems being typical of an Arabic owned and governed institute in the UAE, where ultimate power of authority and veto remained the remit of the Emiratis nationals (Figure 17). The Gazelle Institute had a Central Services (CS) Administration division located separately from all the Colleges, staffed by the Chancellor as a member of the ruling family, an Emirati Vice Chancellor, Academic Program Deans for the whole system and a centralized Institutional Effectiveness unit. A policy committee comprising the Directors from the 12 Colleges worked closely with CS, meeting monthly to discuss strategic issues and directions for the whole system. Directorship and senior management at the College level was drawn from a diverse expatriate workforce responsible for College operations. At the time of the research project there were two colleges (one male and one female) in six out of the seven Emirates and total student numbers at the College involved in this research were approximately 2,500 females compared with system-wide numbers approximating 16,500. The College's management structure included the Director and two Academic Deans as the senior management team', and a College management team on which all Academic Chairs and Support Services managers sat together with the senior management team.

The aim for educational delivery was at western standard 'best practice', which is a huge shift from what was on offer prior to the federation of the UAE. The 'traditional' understanding of education prior to 1971 was predominantly that of religious education, with behavioural norms and values being instilled in children through adult mentoring and articulation of expectations from a young age. Formal education per se was quite expensive and many Emirati families needed their children to assist in family fishing and farming businesses, with lessons in the Quran sometimes being the only form of 'formal' education. The role of teacher was usually assumed or delegated to the Imam and it was not unusual for teaching of mathematics to be interspersed with lessons from the Holy Quran. It was not until 1953 that 'modern' schooling appeared with the assistance of the British and later Kuwaitis, with wealthier nationals being given the opportunity to study overseas with all expenses paid by the UAE government. 


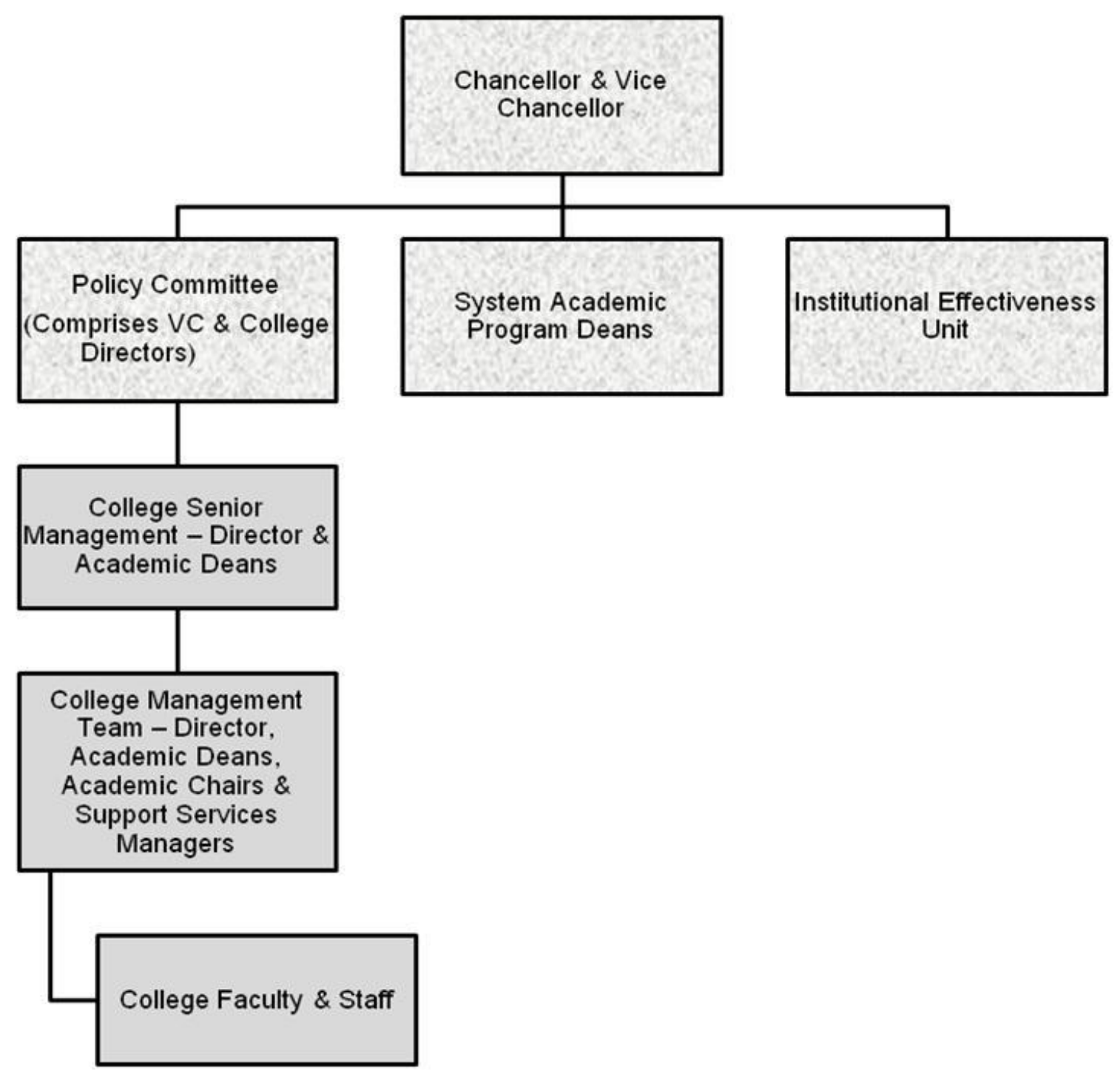

Figure 17 Organisational structure

The Gazelle Institute was established 20 years ago to provide higher educational programs for the country's national Emirati students. Whilst the organization faced similar challenges to educational institutes in the 'west' where change is constant, rapid and complex, additional challenges in the UAE include the nation's dependency on an unstable and mobile expatriate workforce drawn from diverse socio-cultural environments. Tensions from the 'meeting' of different cultural minds in the workplace are a constant. Expatriate teachers educated in countries with their own particular cultural traditions arrive to work with national students who are connected with a quite disparate 
cultural history. Respecting students' heritage whilst helping them juggle the realities of a rapidly evolving, complex and ambiguous social world, creates ongoing challenges. This is compounded by the fact that many of the national students were the first generation in their family to receive higher education, with family conflicts and educational expectations frequently being at odds with the realities of western-driven education.

Students and the prevailing Arabic system of governance at the Gazelle Institute strongly influenced and defined work practices in relation to family, with the concept of self being difficult to separate from significant others within their 'group', whether that be family, friends, relatives or region. The Quran is purported to reinforce the strength of family connections within a collectivist society, with patriarchal dominance and authority of the male figure being the norm. This extends to social and organizational settings where high power distance and acceptance of the leader's authority is unquestioned due to Islamic advocation that "...people should not be critical of decisions and application of their superiors...[rather they should] ...obey them without questions" (Kabasakal and Bodur 2002 p.47). Thus inequalities of power distribution together with family collectivism are strong determinants of social relations and practices, potentially creating tensions within an educational environment attempting to benchmark at western standards which encourages critical enquiry, challenging of authority and independent thought. In addition, western expatriate teachers tend to base their practice on individualist (as opposed to collectivist) performance orientations, and whilst management is still predominantly male, the valuing of gender equality is aspired to more so than patriarchal dominance. Organisational learning through inquiry assumes future focused and goal directed behaviour. This created another challenge at the Gazelle Institute due to Arabic cultures scoring low in future orientation behaviour with planning for the future being considered insignificant (Hofstede 2001; Kabasakal and Bodur 2002). This is in part due to the Islamic belief in God-determined fate and the collectivist reliance on significant others and interdependent relationships to make group rather than individual decisions.

Schooling in the UAE for the national population over the past 40 years has predominantly been imported, with all educational curriculum, textbooks and even building design being drawn from overseas (Heard-Bey 2004). While junior and secondary schools have imported the majority of their teachers from other Arab countries, in the last 15 or so years the Gazelle Institute as for other higher education institutes has sought to develop and implement western-modelled education, encouraging Anglo originated expatriate teachers to educate Emirati nationals using the medium of English for all tuition. Increasingly national Emiratis are being equipped with the knowledge and skills for the workforce due to "...an aggressive tertiary education policy..." (Heard-Bey 2004 p.xxxii). However, two of the challenges for those responsible for education systems in the UAE have been to ensure that educational policy is dovetailed with political and economic policies so graduates are greeted 
with sufficient and appropriate employment opportunities, together with ensuring that students are receiving education at standards equivalent to their student counterparts in the western world.

One attempt at benchmarking educational standards was the seeking of international accreditation both for the Institution and for educational programs developed within the system. Integral to these processes was the establishment and implementation of College Quality Assurance systems to improve organizational effectiveness and measure teaching and learning delivery.

\subsubsection{The Problem}

As described in Section 3.2 the directive to implement a Quality Assurance (QA) program in each of the twelve colleges, was imposed by the Central Administrative core of the organization as integral to a broader institutional accreditation initiative. The standards and key criteria were pre-determined with Colleges being responsible for establishing QA policies and procedures to reflect an operational QA system and compliance with standards. Allocation of the human resources necessary for QA was the remit of the individual colleges, with it being assumed that once systems were up and running, the effectiveness of the organization could be measured.

As the Academic Chair of a smaller department, I was appointed as project manager and QA coordinator for the College in addition to my normal duties for the Academic Year (AY) of $2005-2006$ (SeeTable 5) which includes research timeline. In the year prior 2004-2005 one of the College Academic Deans was responsible for College QA, attending system-wide meetings and communicating requirements to the College's management team. As I had been involved in program QA for the first college audit under the new system in the 2003-2004 AY, I was frequently called upon to give advice and participate in any QA meetings at the College in 2004-2005 AY. In addition I represented the College at system-wide meetings when the Dean was unable to attend. As mentioned, in the outset additional funding had not been allocated for QA system establishment in the Colleges so that work break down structures needed to be aligned with organizational break down structures within the functional hierarchy. Existing staff therefore assumed responsibility for necessary work in addition to normal duties and responsibilities. Throughout 2004-2005 there appeared no clear pathway for QA within the College. It was not a key issue at the management meetings with many assumptions being made about what was happening. From my perspective as an Academic Chair, I was confused as to QA requirements at the College level, and this was despite being more involved than other management team members in system-wide QA meetings. Information conveyed at College management meetings conflicted with system-wide QA meetings I had attended. Other information seemingly was being withheld or not shared, either due to lack of appreciation for the importance or failure to articulate priorities. 
College management team members were responsible for ensuring implementation of the $Q A$ system within their departments, including all data collection for provision of evidence, analysis and reporting. My role as QA co-ordinator involved developing a timeline in accordance with CS directives, overseeing consistency in standards, identifying system and College process gaps, developing a centralized College knowledge storage system and acting as a reference point for QA communication between CS and the College. In addition I had the responsibilities of QA for my own department, and as the College representative was on a number of system-wide committees where members from other Colleges were also grappling with processes necessary to ensure Key Criteria policy and procedural consistency.

There were approximately 23 management team members and 140 academic faculty at the College involved in this research project, with employees originating from 13 predominant countries (Canada, Australia, New Zealand, USA, UK, Ireland, South Africa, Egypt, India, Sudan, Pakistan, Philippines and Sri Lanka), plus smaller numbers from other Gulf states, Lebanon, Syria, Jordan and Palestine. In addition, non-academic staff totalled approximately another 60 staff, with their country of origin on a system-wide basis varying across 42 other countries.

Between August 2005 and January 2006 QA was a consistent agenda item on management and academic management meetings. Despite the overall 'awareness' of QA within the College, there appeared to be little progress in the establishment of college processes, nor support for coordinated implementation. Some departments continued doing their 'own thing', others tried a piece-meal attempt at QA, other departments seemed to make a concerted effort (particularly those that were due for initial audit), whilst other departments, despite sitting through management meetings and discussing the issues, seemingly did nothing. In discussing the situation with numerous stakeholders, both within the college and within the college system, some of my frustrations as QA co-ordinator were shared by many. I had my own particular perceptions of the problematical situation, but conflicting accounts of what the 'problem' was perceived to be warranted further exploration if constructive change and progress was to be made possible.

The methodological process comprised four cyclic iterations, with two utilizing SSM to unravel the process of inquiry (SSMp), one learning cycle working through the content of the problematical situation (SSMc) and a $4^{\text {th }}$ cycle that returned to a planning approach (SSMp) as portrayed inTable 5. The third cycle was the most 'complete' with discussions resulting in accommodated desirable and feasible change being defined (Table 9). I was unable to oversee implementation of suggested changes due to the sudden termination of my employment contract prior to implementation, reasons for which will be discussed in Chapter 5. Cycle 4 comprised continuation of the process outside the organization, with further evolution of the rich picture. Three predominant areas of concern were shared by most remaining 'problem owners' as identified through informal meetings 
and discussions. Further recommendations for desirable and feasible change were defined as possible actions to be implemented in another organization (Table 10). 
Table 5 The Learning Cycles in Action

\begin{tabular}{|c|c|c|c|c|c|}
\hline \multirow[t]{2}{*}{$\begin{array}{l}\text { Learning Cycle } \\
\text { Actions }\end{array}$} & Timeline & $\begin{array}{r}\text { Aug '05 - Jan'06 } \\
\rightarrow \rightarrow \rightarrow \rightarrow \rightarrow \rightarrow \rightarrow \rightarrow\end{array}$ & $\begin{array}{r}\text { Jan '06-Nov '06 } \\
\rightarrow \rightarrow \rightarrow \rightarrow \rightarrow \rightarrow \rightarrow \rightarrow\end{array}$ & $\begin{array}{c}\text { Dec '06-Jan '07 } \\
\rightarrow \rightarrow \rightarrow \rightarrow \rightarrow \rightarrow \rightarrow \rightarrow\end{array}$ & $\begin{array}{r}\text { Jan'07 - Jun '07 } \\
\rightarrow \rightarrow \rightarrow \rightarrow \rightarrow \rightarrow \rightarrow\end{array}$ \\
\hline & Academic Year & 2005-2006 Sem I & $\begin{array}{l}\text { 2005-2006 Sem II } \\
\text { 2006-2007 Sem I }\end{array}$ & 2006-2007 Sem I & 2006-2007 Sem II \\
\hline $\begin{array}{l}1-4=\text { Learning Cycle } 5 \\
=\text { Action for change }\end{array}$ & Stage & $\begin{array}{c}\text { Cycle } 1 \text { SSMp } \\
\text { Planning the approach }\end{array}$ & $\begin{array}{c}\text { Cycle } 2 \text { SSMp } \\
\text { Planning the intellectual process }\end{array}$ & $\begin{array}{l}\text { Cycle } 3 \text { SSMc } \\
\text { Addressing the content of the problem } \\
\text { situation }\end{array}$ & $\begin{array}{c}\text { Cycle } 4 \text { SSMp } \\
\text { Planning the approach }\end{array}$ \\
\hline $\begin{array}{c}\text { Action } 1 \\
\text { The perceived } \\
\text { situation }\end{array}$ & 1. $\quad$ Finding out & $\begin{array}{l}\text { QA system and priorities presented } \\
\text { at management meeting }\end{array}$ & $\begin{array}{l}\text { Rich picture of situation. Informal } \\
\text { \& formal discussions, individual } \\
\text { conversations, meetings }\end{array}$ & $\begin{array}{l}\text { Evolving rich picture. Analysis 1, Formal } \\
\text { interviews con't. Analysis } 2 \& 3 \\
\text { concurrent }\end{array}$ & $\begin{array}{l}\text { Evolving rich picture. Continued } \\
\text { reflection, incorporated further } \\
\text { Analyses } 2 \& 3\end{array}$ \\
\hline \multirow[t]{3}{*}{$\begin{array}{l}\text { Action } 2 \\
\text { Purposeful } \\
\text { activity }\end{array}$} & $\begin{array}{l}\text { 2. } \begin{array}{l}\text { Expressing the } \\
\text { problem situation }\end{array}\end{array}$ & Discussion of situational needs & $\begin{array}{l}\text { Stakeholders identified. Problem } \\
\text { situation discussed. Initial social } \\
\& \text { political analyses }\end{array}$ & $\begin{array}{l}\text { Individual models of purposeful activity } \\
\text { identified as transformational statements; } \\
\text { Generic model updated }\end{array}$ & $\begin{array}{l}\text { Three predominant problem areas of } \\
\text { concern identified; questions emailed } \\
\text { to owners }\end{array}$ \\
\hline & $\begin{array}{l}\text { 3. Formulating the } \\
\text { root definition }\end{array}$ & Statement about activity required & $\begin{array}{l}\text { Statement about activity } \\
\text { required. Root definition (RD) } \\
\text { developed }\end{array}$ & PQR, RD, CATWOE, 3 Es & Responses contributed to evolving RD \\
\hline & $\begin{array}{l}\text { 4. Building conceptual } \\
\text { models }\end{array}$ & $\begin{array}{l}\text { Action plan developed based on } \\
\text { statement }\end{array}$ & $\begin{array}{l}\text { Models of action developed } \\
\text { based on initial RD }\end{array}$ & $\begin{array}{l}\text { Five models of purposeful activity being } \\
\text { modelled }\end{array}$ & $\begin{array}{l}\text { Informal meetings and discussions } \\
\text { outside workplace. Models revised }\end{array}$ \\
\hline $\begin{array}{c}\text { Action } 3 \\
\text { Comparison - } \\
\text { structured discussion }\end{array}$ & $\begin{array}{l}\text { 5. Comparing models } \\
\text { and perceived real } \\
\text { world }\end{array}$ & $\begin{array}{l}\text { Meeting involved discussion \& } \\
\text { identification of challenges in } \\
\text { implementation compared with } \\
\text { planned action }\end{array}$ & $\begin{array}{l}\text { Matrix of real situation with } \\
\text { desirable \& feasible changes } \\
\text { based on initial interviews }\end{array}$ & $\begin{array}{l}\text { Structured discussions using models to } \\
\text { compare with generic model; reflection } \\
\text { up on real, perceived situation }\end{array}$ & $\begin{array}{l}\text { Compared and made changes to } \\
\text { recommendations }\end{array}$ \\
\hline $\begin{array}{l}\text { Action } 4 \\
\text { Defining action to } \\
\text { improve }\end{array}$ & $\begin{array}{l}\text { 6. Debating, defining } \\
\text { changes }\end{array}$ & Reprioritized action & Reprioritized action defined & $\begin{array}{l}\text { Recommendations developed for action } \\
\text { based on discussions of what changes } \\
\text { were desirable, feasible. Defined actions. } \\
\text { Employ ceased }\end{array}$ & $\begin{array}{l}\text { Discussed proposed action with } \\
\text { workers in different work setting }\end{array}$ \\
\hline $\begin{array}{l}\text { Action } 5 \\
\text { action \& Reflection }\end{array}$ & 7. Taking action & $\begin{array}{l}\text { Development and implementation } \\
\text { commenced }\end{array}$ & Action taken & Reflect on defined actions & $\begin{array}{l}\text { Recommended trial implementation in } \\
\text { similar work environment }\end{array}$ \\
\hline
\end{tabular}




\subsection{The Learning Cycles in Action}

\subsubsection{Introduction}

The following section presents the process of inquiry working through the different cycles of action as described above (Table 5). The first two cycles followed a pattern of action learning, but were not as structured or as formal as Cycle 3, which used SSM to inquire into the content of the problematical situation to seek accommodations for making improvement. Therefore the first two cycles (SSMp) will be described and discussed more briefly, supported by diagrammatic portrayals of the perceived situation. The third cycle (SSMc) is described as involving the defined action, and the fourth cycle (SSMp) provides an overview of the enquiry process subsequent to leaving the College.

\subsubsection{Cycle 1}

The Academic Year (AY) runs from August of one calendar year until June the following. Prior to the 2003 - 2004 AY, Quality Assurance (QA) within the Gazelle Institute was completed by Academic Programs across the system rather than by Colleges. The first attempts at College based QA was in alignment with the move toward system-wide Institute Accreditation and began in the 2003-2004 AY. Two program departments were selected to benchmark their program delivery against system wide Key Criteria. I was Academic Chair of one of the departments required to provide evidence and a report by end of 2003-2004 AY. The first round of QA involved a large amount of administrative time and numerous frustrations through the year, primarily due to the lack of system wide processes to support College action and implementation.

Throughout the following 2004-2005 AY, two other program areas were to be audited. Despite one of the College's Academic Deans being responsible for College QA, I found other Academic Chairs continually sought guidance from me about QA processes based on my experience the year prior. I was also asked to attend numerous College and system-wide QA meetings to provide advice and represent the College when the Academic Dean was unavailable.

In the 2005-2006 AY I was appointed as College QA co-ordinator in addition to my functional role as Academic Chair. Integral to this position of College QA co-ordinator was attendance and participation at system-wide meetings and working parties. Other members of these committees were full-time appointed QA co-ordinators - I was the only College being represented that did not have a dedicated QA staff member. 
The first cycle of project learning action began in August 2005 with the beginning of the new AY (see Table 5. The 'Finding out' stage about the perceived problematical situation involved reflections on my experience the previous AY, discussions with the other Academic Chairs about the challenges they faced in submitting their program report, informal discussions with faculty and other management team members about their perceptions of QA within the College, and more formal discussions at Management and Academic team meetings. (Figure 18)

I had agreed to act as the College QA representative for a number of reasons. Firstly because I enjoyed working through systems to make improvements, secondly because I felt there had been little progress over the past couple of years and thirdly because I wanted to see what could be done to make a difference.

On my appointment as College QA coordinator, I reviewed previous audit reports and existing college systems, identified gaps and then presented my findings to the College Management team in October 2005. The summarized report recommendations were given to the senior management team (Director and Academic Deans) (see App 1), together with a summarized system QA update report (see App 2). A Gantt chart of a proposed action timeline from October 2005 to June 2006 was tabled at the next full College management meeting (App 3) for discussion. 


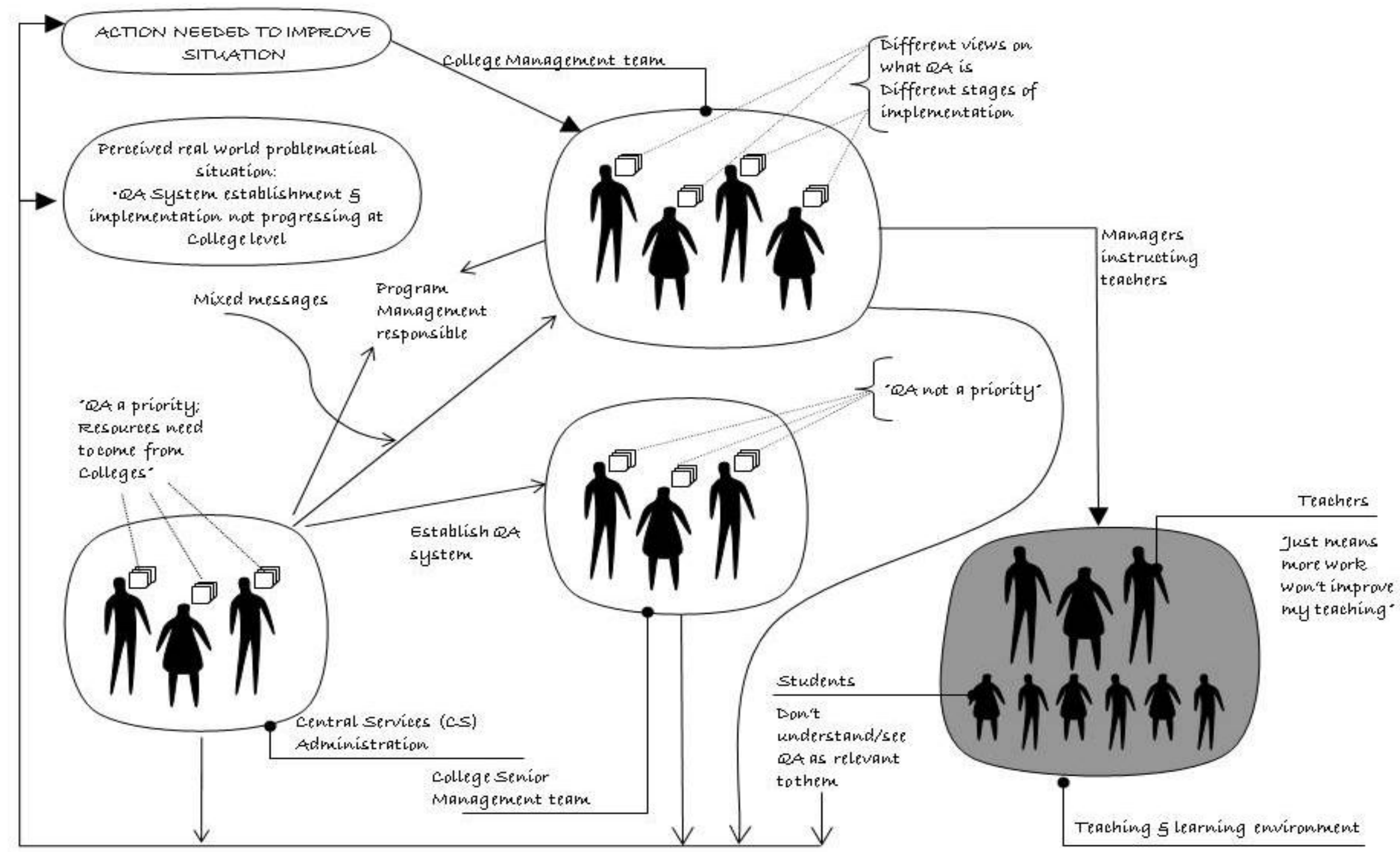

Figure 18 Cycle One - The Finding Out (A) 
The main issues identified and agreed upon actions from this College management team meeting included:

- QA coordinator to review and confirm College QA processes at next management team meeting;

- QA coordinator to review QA processes to extract administrative;

- Academic Chairs to identify and forward to QA coordinator any processes used at Program level currently not included in College QA process list;

- QA coordinator to reformat College QA processes into CS template (indicating mechanism, process, evidence of process and staff responsible);

- Management team members to review CS 'web-look' version once available.

These issues generated discussion about situational needs and possible activities required, providing new perspectives on the issues to incorporate into my perception of the problem situation to date (Figure 19).

Meetings between November 2005 and early 2006 involved encompassed:

- Working through data checklists developed from a CS template (App 4);

- Guiding Academic Chairs in QA processes and assisting in identifying staff responsibilities (App 5);

- Discussing IT Data Management system and data storage needs and responsibilities (App 6).

Outcomes from these meetings were compared with action plans and priorities identified in October, with amendments being made after reflecting on emergent needs and issues throughout the remainder of the 2005-2006 AY.

Identified actions were implemented, adding to the development of a more detailed and 'richer' picture of the problematical situation (Figure 20). Seven Program Quality Assurance (PQA) Key Criteria (KC) had being established system wide but there seemed to be some differences in understanding about processes required or how autonomous Colleges could be. Work that was being implemented one week frequently became 'undone' the next as new directives were issied externally and internally to the College. This created frustrations for Academic Chairs and other management team members who were already having difficulties finding time to implement any new changes over and above their usual duties. I also felt I was going round in non-productive circles despite what appeared to be realistic action plans which had been developed through extensive discussions with stakeholders concerned. The rudiments of an 'action research' project seemed to lie in front of me, with my enrolled status in the Doctorate in Project Management program appearing to be a logical vehicle for trying to bring about improvements to the situation (Table 6). 


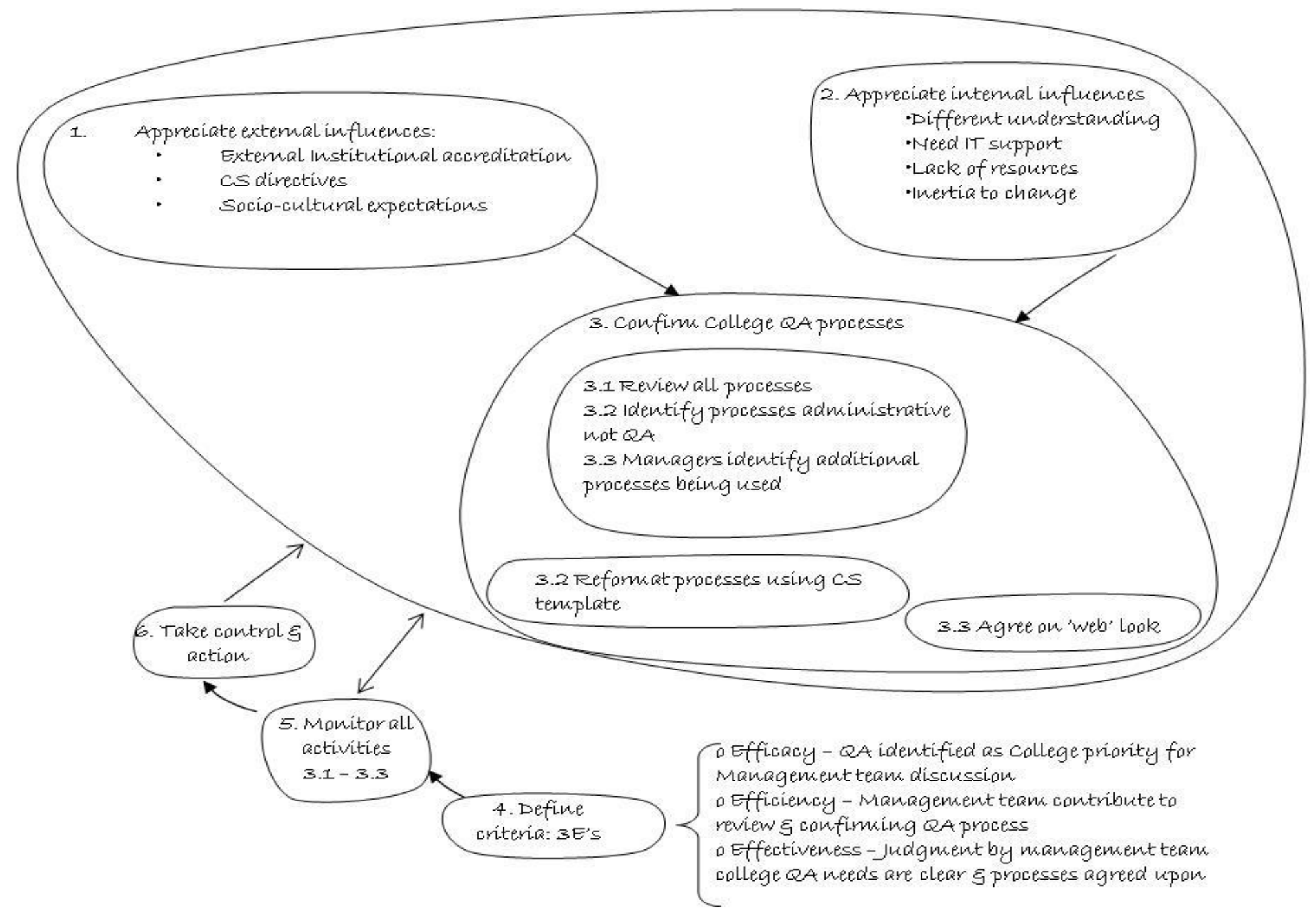

Figure 19 Cycle One - The Finding Out (B) 
Table 6 Cycle One Action Matrix (structure modelled on Checkland \& Scholes 1990)

\begin{tabular}{|c|c|c|c|c|c|}
\hline $\begin{array}{l}\text { Activities from the } \\
\text { model }\end{array}$ & How the activity is done & $\begin{array}{l}\text { Measures of } \\
\text { performance }\end{array}$ & $\begin{array}{l}\text { Information } \\
\text { needed }\end{array}$ & $\begin{array}{l}\text { Information } \\
\text { support } \\
\text { provided by: }\end{array}$ & $\begin{array}{l}\text { Information gaps and } \\
\text { opportunities }\end{array}$ \\
\hline $\begin{array}{l}\text { Develop and distribute } \\
\text { data checklist } \\
\text { responsibilities to relevant } \\
\text { stakeholders }\end{array}$ & $\begin{array}{l}\text { QA coordinator uses CS template to } \\
\text { develop checklist using KCs } \\
\text { QA coordinator to distribute and discuss } \\
\text { checklist to all Academic Chairs }\end{array}$ & $\begin{array}{l}\text { Checklist developed and } \\
\text { distributed }\end{array}$ & $\begin{array}{l}\text { CS Key Criteria } \\
\text { System wide agreed } \\
\text { process list } \\
\text { College agreed } \\
\text { processes }\end{array}$ & QA coordinator & $\begin{array}{l}\text { Liaise with system wide } \\
\text { committees for useful } \\
\text { processes }\end{array}$ \\
\hline $\begin{array}{l}\text { Review program } \\
\text { responsibilities for data } \\
\text { management by program }\end{array}$ & $\begin{array}{l}\text { QA coordinator to develop and } \\
\text { distribute responsibility checklists to } \\
\text { College management team } \\
\text { Academic Chairs to discuss with } \\
\text { program faculty perceptions of data } \\
\text { needs }\end{array}$ & $\begin{array}{l}\text { Checklist developed and } \\
\text { distributed } \\
\text { Academic Chairs identified } \\
\text { program responsibilities } \\
\text { Academic Chairs report } \\
\text { faculty data needs }\end{array}$ & $\begin{array}{l}\text { Developed checklist } \\
\text { College agreed } \\
\text { processes } \\
\text { Data storage } \\
\text { system }\end{array}$ & $\begin{array}{l}\text { QA coordinator } \\
\text { Management team } \\
\text { IT \& Ed Tech } \\
\text { departments }\end{array}$ & $\begin{array}{l}\text { Liaise with other Colleges to } \\
\text { investigate data storage } \\
\text { systems }\end{array}$ \\
\hline $\begin{array}{l}\text { Allocate data management } \\
\text { responsibilities to program } \\
\text { staff }\end{array}$ & $\begin{array}{l}\text { Academic Chairs to identify and } \\
\text { delegate data collection, analysis and } \\
\text { entry responsibilities to program faculty }\end{array}$ & $\begin{array}{l}\text { Faculty aware of } \\
\text { responsibilities } \\
\text { Completed checklists with } \\
\text { staff responsible returned to } \\
\text { QA coordinator }\end{array}$ & $\begin{array}{l}\text { Developed checklist } \\
\text { Faculty allocation }\end{array}$ & $\begin{array}{l}\text { Academic Chairs } \\
\text { QA coordinator }\end{array}$ & $\begin{array}{l}\text { Methodology to be discussed } \\
\text { at management team } \\
\text { meetings for sharing of ideas, } \\
\text { solutions, problems and } \\
\text { progress }\end{array}$ \\
\hline $\begin{array}{l}\text { Identify QA data storage } \\
\text { needs }\end{array}$ & $\begin{array}{l}\text { QA coordinator to investigate other } \\
\text { college systems } \\
\text { QA coordinator to liaise with Academic } \\
\text { Chairs to identify data storage needs } \\
\text { QA coordinator to liaise with IT \& Ed } \\
\text { Tech departments to advise on data } \\
\text { storage needs \& responsibilities }\end{array}$ & $\begin{array}{l}\text { Data storage system } \\
\text { established, used and } \\
\text { communicated to } \\
\text { management team }\end{array}$ & $\begin{array}{l}\text { Estimated output } \\
\text { from data analyses } \\
\text { Range of IT systems } \\
\text { which can support } \\
\text { QA } \\
\text { Gaps in IT systems } \\
\text { that need filling } \\
\text { Resources available }\end{array}$ & $\begin{array}{l}\text { Academic Chairs \& } \\
\text { QA coordinator } \\
\text { IT Department } \\
\text { IT Department } \\
\text { College Director }\end{array}$ & $\begin{array}{l}\text { Opportunity to use system } \\
\text { wide data storage system for } \\
\text { consistency and better } \\
\text { utilization of resources }\end{array}$ \\
\hline $\begin{array}{l}\text { Develop Knowledge } \\
\text { Management strategies }\end{array}$ & $\begin{array}{l}\text { QA coordinator, management team and } \\
\text { IT department discuss and identify } \\
\text { factors affecting KM systems; needs \& } \\
\text { limitations to be identified }\end{array}$ & $\begin{array}{l}\text { KM system established and } \\
\text { new processes implemented } \\
\text { All relevant stakeholders } \\
\text { informed of new systems }\end{array}$ & $\begin{array}{l}\text { IT capacity } \\
\text { Available software } \\
\text { User needs }\end{array}$ & $\begin{array}{l}\text { IT department } \\
\text { Ed Tech } \\
\text { Department } \\
\text { QA coordinator }\end{array}$ & $\begin{array}{l}\text { Draw upon other Colleges } \\
\text { within the system which may } \\
\text { have functional KM strategies } \\
\text { and systems established }\end{array}$ \\
\hline
\end{tabular}




\subsubsection{Cycle 2}

The second cycle commenced in January/February 2006, $2^{\text {nd }}$ semester of the 2005-2006 AY. Figure 20 indicates the evolving rich picture description of the problematical situation.

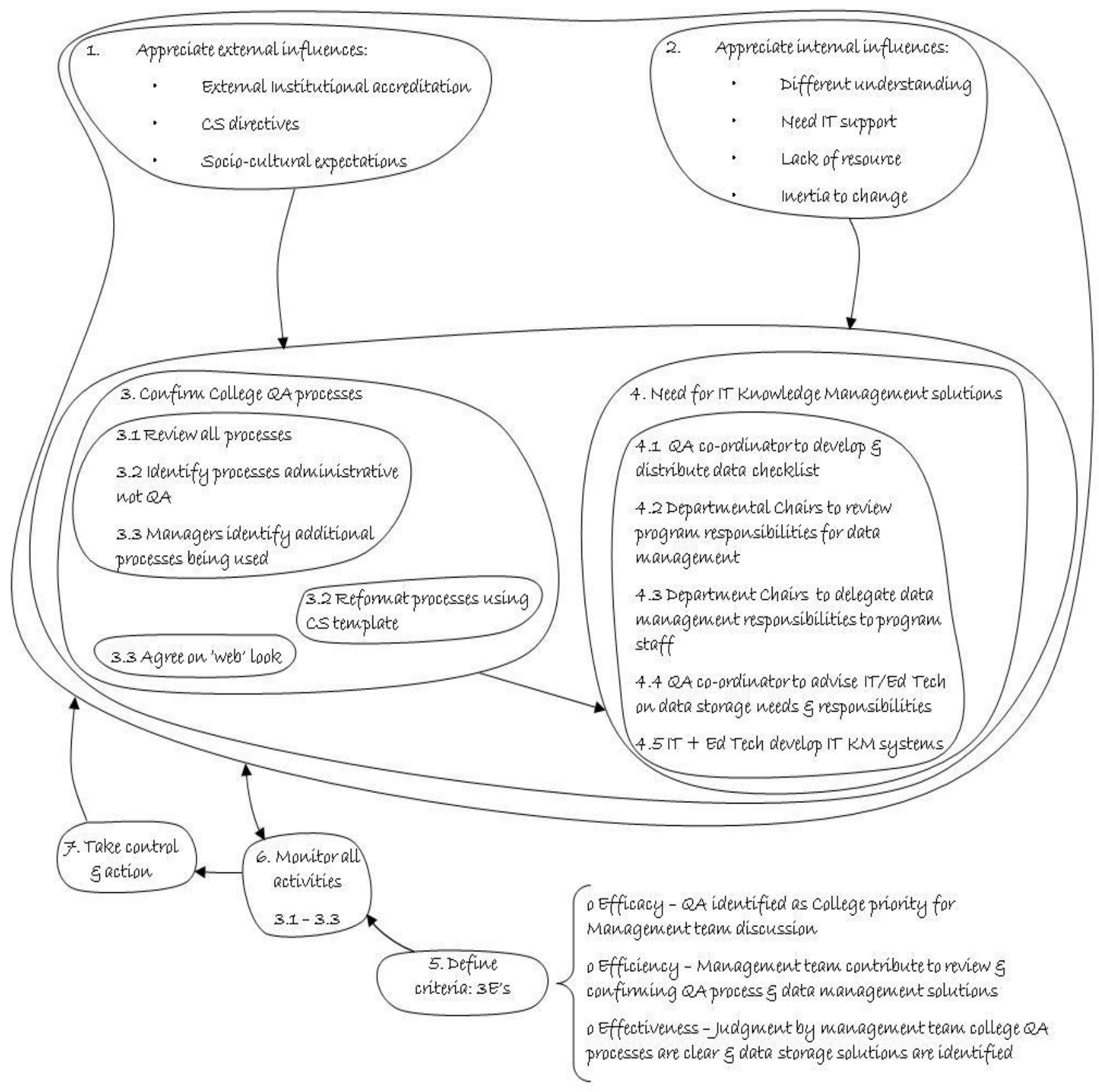

Figure 20 Cycle 2 - The evolving picture

Further informal and formal discussions were held with management team members (i.e. Academic and Service Department Chairs), faculty (from my department and those in other departments where I was invited to attend meetings to explain PQA requirements) and system wide committee 
members. Reflections were made upon these conversations, comparisons made with issues raised the previous semester and consideration began to be given to the social and political context of the QA project.

After discussing my perceptions of the problematical situation with the College Director given reflections on the data accumulating from the 'finding out', approval was given to implement the research in the College and I then proceeded with a DPM project application. The formal ethics application was subsequently submitted to RMIT in September 2006 and after some refinement was approved in December 2006. Meanwhile, through the period of February 2006 to November 2006 the SSMp cycle of planning the intellectual process continued and a number of interviews were commenced.

\section{Initial Analysis 1, 2 and 3}

The initial Analysis 1 involved giving thought to the roles of stakeholders, identifying who had 'caused the intervention' (client) and who were 'concerned or affected by the situation' (problem owners). As I was the researcher 'conducting the investigation' I identified myself as 'participant' or 'problem solver', however, being a departmental manager, member of the College management team, the College QA co-ordinator and member of system-wide QA committees, subsequently meant I held multiple roles, and was in reality a 'problem owner' as well. Although PQA implementation and the decision to move with institute accreditation involved many players, the 'client' has been identified as the Central Services (CS) administration, which includes the Chancellor and ViceChancellor, the policy committee (comprising Directors of all individual Colleges), the system Academic Program Deans and the Institutional Effectiveness Unit (Figure 17). Again, although faculty and students were also affected by the situation, the 'owners of the issue' as 'probelm owners' are identified as the College Management team as they had the potential to improve the problematic situation.

Socially (Analysis 2), senior management (College Director and two Academic Deans) overlapped in their roles and responsibilities through fulfilling directives from CS, but they were also members of the College management team which was responsible for educational delivery to the Emirati students (Figure 17). In turn, most management team members primarily held academic chair positions or if in support services, a supervisory management position; but informally it was common for staff to hold multiple roles including teaching, clinical placement, coordinating campus activities and industry liaison. Most academic chairs also spent a large proportion of their time in addressing student issues, family advice and behaviour management. These roles and/or responsibilities related to norms and expectations of educational delivery by the Emirati families, the maturity level of students entering the College and cultural perceptions of the role of 'teachers' in an Arabic society 
(Figure 21). These factors were identified in the interviews and will be discussed further in the Discussion chapter.

Management team members needed to 'fit' the behavioural norms of the College defined in policy terms as 'culturally appropriate dress and behavior demonstrating respect for the Arabic culture and Islamic religion'. Norms are also established by the leader or Director of each College according to their expectations and background. Families and students also influence what behavior is acceptable, with the need for educators to compromise, adapt and remain sensitive to the local culture. Norms of behavior also vary according to who visits the college, with a respectful and conservative approach being expected particularly if there is a delegation involving the Chancellor or other Emirati dignitaries. Gender differences in appointments reflect the socio-cultural context, with all College Directors being male. Methods of problem solving or raising issues of concern is also socially patterned, with families expecting to have access to the Director or 'leader' of the college, thus an 'open-door' approach to college management existed. This Arabic democratic approach was further evidenced with family members being able to contact the VC directly if they chose, or if preferred, discussion of issues of concern could be raised with the Chancellor directly at his palace majilis. Values in relation to educational delivery were drawn from CS expectations of quality western-derived programs, but in Cycle 3 emergent variations about perceived priorities from managers of western origin revealed a number of differences in understandings and expectations.

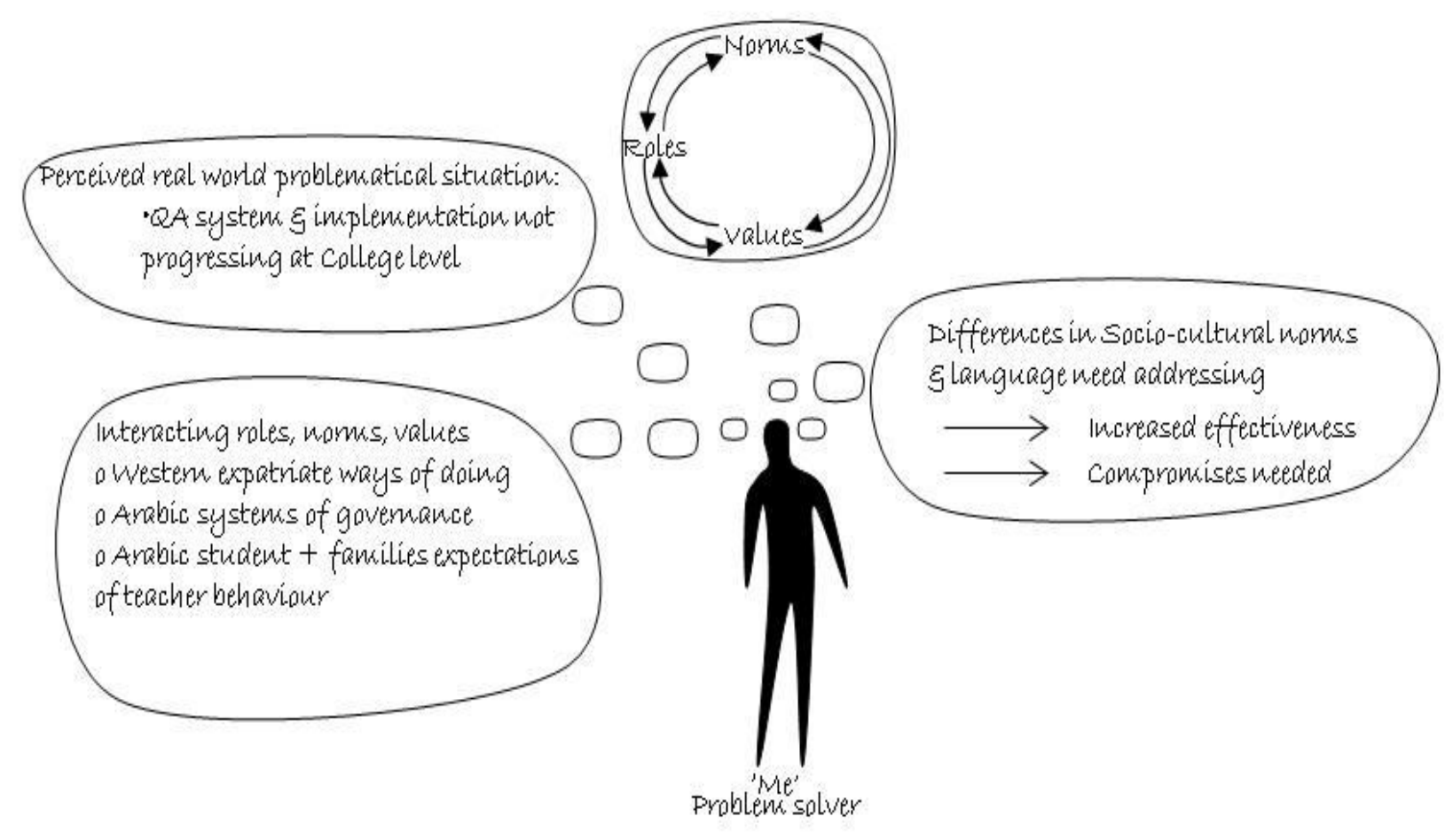

Figure 21 Cycle 2 - The initial social analysis 
Political Analysis (Analysis 3) will be raised in more detail in Cycle 3 and discussed in Chapter 5. However, on initial analysis, the political situation describing the spread of 'power' and the feasibility of different actions is critical to this project (Figure 22). The complex nature and sources of power are inseparable from cultural factors. Ultimate power of authority lies in the hands of the national Emirati population. Ownership and funding for the majority of College activities and all education is sourced through private royal and government funding. The Emirati students do not pay fees and all materials and resources are provided free of charge in alignment with the vision of the UAE's first ruler, Sheikh Zayed bin Sultan Al Nahyan, who instigated a major investment in education stating the Emirati youth are the 'real wealth of a nation'. Staff appointments are approved at the College Director level, however, all employee contracts also need to pass central personnel committee perusal, with final sign off being the remit of the VC. This multi-layered approach has been raised by a number of 'problem owners' as a limiting factor to college autonomy, together with creating feelings of disempowerment and job insecurity. Expatriate contracts are of limited time duration, with quite stringent requirements if the employee chooses to end the agreement. Expatriates enter the UAE knowing there is an absence of government legislation to 'protect' non-national employees. Inequalities in employment contracts are a reality that creates discrepancies in the distribution of power, with nationality and reputation being major determinants in issues of employment.

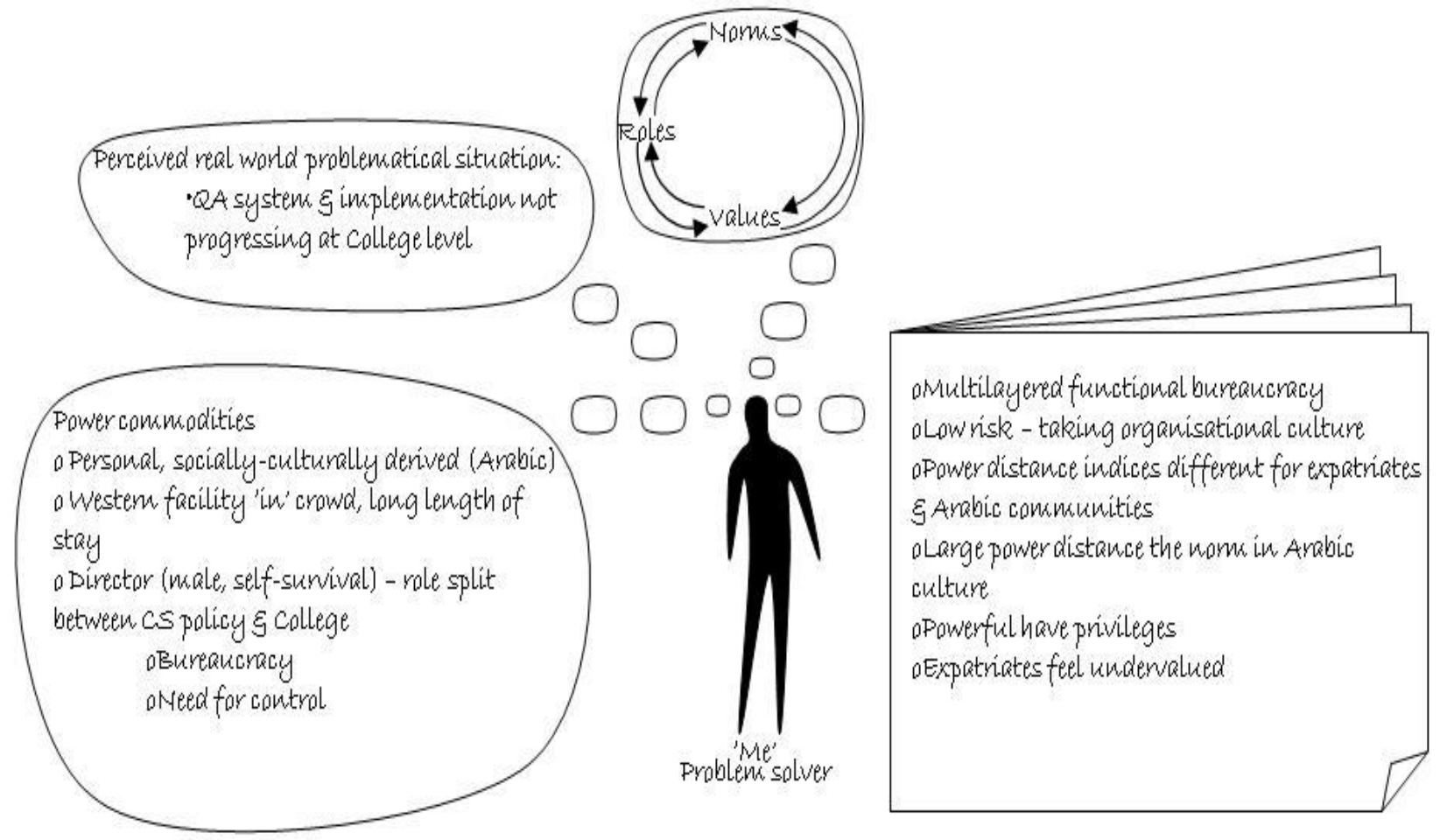

Figure 22 Cycle 2 - The initial political analysis 


\section{Rudimentary Root Definition (RD) as statement of activity}

An initial RD as an introductory statement in relation to QA activity implementation was developed from the first two planning cycles:

"A College QA system which needs to be consistent internally across programs and departments, aligned with system-wide processes, and serves as an accurate measure to sustain teaching and learning effectiveness"

\section{Models of action developed to present at management meeting}

These were identified as management meeting action items as agreed 'desirable' changes:

- The need for management team members to confirm QA is a College strategic priority;

- To agree upon the development and identification of clear goals to underpin the QA action plan 2005-2006 and beyond;

- Identify available resources and develop College QA system, including:

- Human resources for planning and co-ordination

- Educational technology support for web page development, department and college

- Administrative support for document preparation, QA administration, data entry.

\section{Considering the real situation with suggested desirable and feasible changes}

After discussing at length the different approaches or 'ways forward' it was decided that using the seven Key Criteria as the guide for process, data gathering, analysis, management and action planning responsibilities would be a useful framework to benchmark both programs and college. As mentioned, the detailed checklists (App 4-5) were for Academic Chairs to use in their programs, whilst the other College Data management responsibility checklist (App 6) was used to guide discussions at several IT data management meetings between October 2005 and March 2006. 


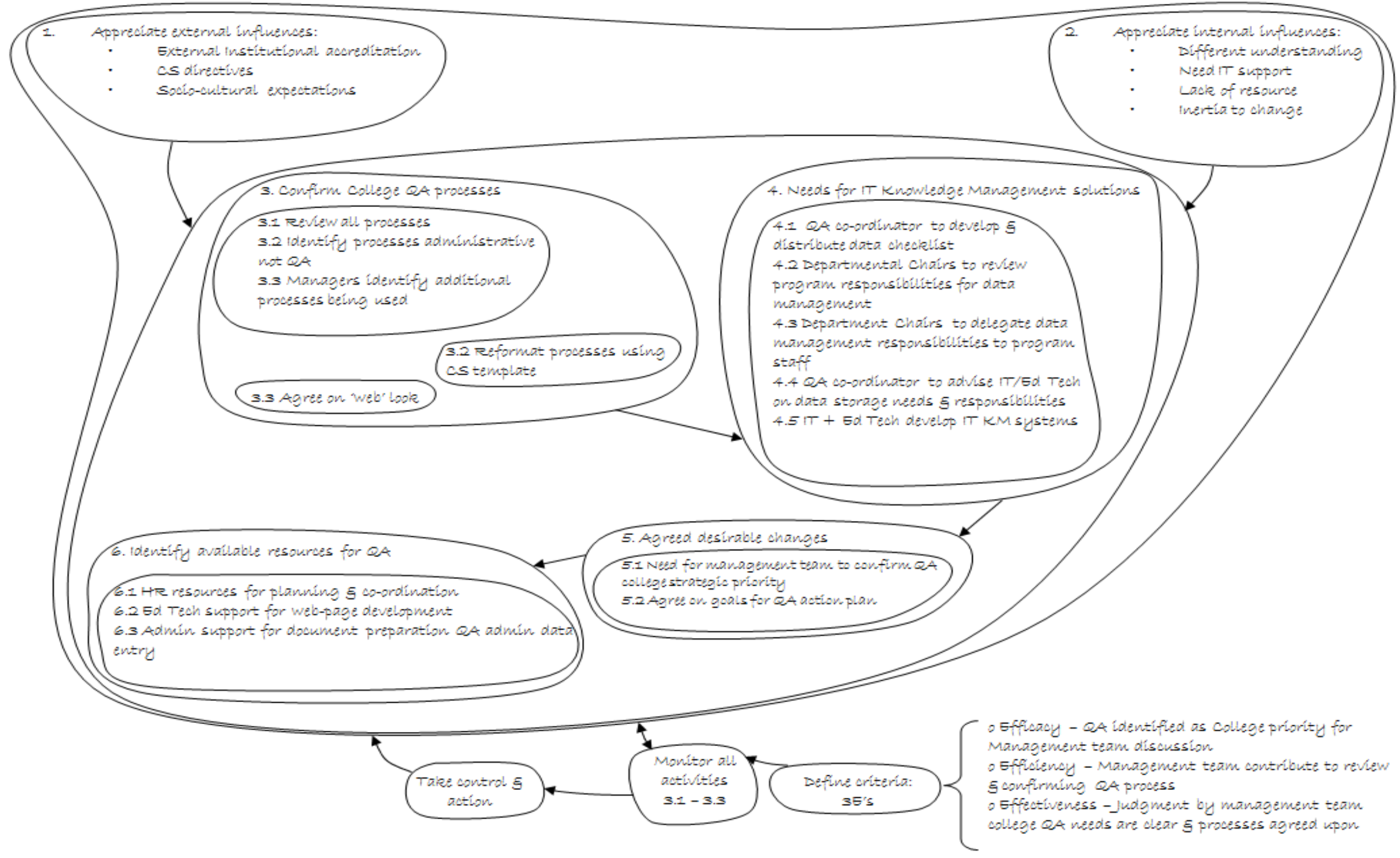




\section{Reprioritized actions defined}

The main issues resulting from discussions on desirable and feasible change based on the KC framework as point of discussion were:

- Educational Technology Department to assume responsibility for establishing data management storage system on a college shared internal drive;

- Data entry, storage, archiving, access, maintenance processes and responsibilities to lie predominantly with management team members;

- Graduate outcome portfolio development, tracking, storage and responsibilities to be assumed by academic and service area departments;

- Consistency in process implementation across programs to be assured through clear College processes, ongoing communication between all managers facilitated by a QA co-ordinator;

- A dedicated College QA coordinator is needed to manage the QA project adequately.

These reprioritized actions were then compiled as a matrix (Table 7) to further discussions and frame necessary actions. 


\section{Table 7 Cycle Two - Action Matrix}

\begin{tabular}{|c|c|c|c|c|c|}
\hline Activities from the model & How the activity is done & Measures of performance & Information needed & $\begin{array}{l}\text { Information support provided } \\
\text { by: }\end{array}$ & $\begin{array}{l}\text { Information gaps and } \\
\text { opportunities }\end{array}$ \\
\hline $\begin{array}{l}\text { Establish a data management } \\
\text { storage system on a college } \\
\text { shared internal drive }\end{array}$ & $\begin{array}{l}\text { Education Technology } \\
\text { Department to co-ordinate } \\
\text { meetings \& discussions with } \\
\text { management team \& QA co- } \\
\text { ordinator to determine needs } \\
\text { Resources allocated so system is } \\
\text { priority Ed Tech project }\end{array}$ & $\begin{array}{l}\text { Folders by program and } \\
\text { department available on } \\
\text { college shared drive; format } \\
\text { consistent across } \\
\text { departments }\end{array}$ & $\begin{array}{l}\text { Data tree structure - by KCs } \\
\text { Confirm KCs } \\
\text { Confirm College Processes } \\
\text { What is working \& not working } \\
\text { in other Colleges }\end{array}$ & $\begin{array}{l}\text { Ed Tech department } \\
\text { CS }\end{array}$ & $\begin{array}{l}\begin{array}{l}\text { College processes not all } \\
\text { confirmed }\end{array} \\
\text { System wide KC and generic } \\
\text { processes still being developed }\end{array}$ \\
\hline $\begin{array}{l}\text { Develop data entry, storage, } \\
\text { archiving, access, maintenance } \\
\text { processes and responsibilities }\end{array}$ & $\begin{array}{l}\text { Management team members } \\
\text { work with QA co-ordinator to } \\
\text { assume departmental data } \\
\text { responsibility. } \\
\text { Departmental managers to } \\
\text { delegate/work with own staff to } \\
\text { commence QA data entry }\end{array}$ & $\begin{array}{l}\text { Data evidence, analysis, } \\
\text { recommendations and } \\
\text { annual report stored in } \\
\text { relevant folders on shared } \\
\text { drive by end of } \mathrm{AY}\end{array}$ & $\begin{array}{l}\text { Dates for survey administration } \\
\text { Confirm processes } \\
\text { Confirm responsibilities }\end{array}$ & $\begin{array}{l}\text { Ed Tech Department } \\
\text { CS Quality effectiveness unit } \\
\text { College QA coordinator }\end{array}$ & $\begin{array}{l}\text { Inconsistencies between CS } \\
\text { System requirements and } \\
\text { College processes } \\
\text { Investigate other College } \\
\text { systems already established; } \\
\text { import what is available }\end{array}$ \\
\hline $\begin{array}{l}\text { Develop graduate outcome } \\
\text { (GO) portfolio development, } \\
\text { tracking, and storage }\end{array}$ & $\begin{array}{l}\text { Responsibilities to be assumed by } \\
\text { academic departments to } \\
\text { incorporate Graduate Outcomes } \\
\text { (GO) portfolios into final year } \\
\text { assessment }\end{array}$ & $\begin{array}{l}\text { Final year students complete } \\
\text { GO portfolio } \\
\text { Soft copy of portfolio for } \\
\text { each student available on } \\
\text { shared drive }\end{array}$ & $\begin{array}{l}\text { IT server capacity } \\
\text { GOs for programs by } \\
\text { departments }\end{array}$ & $\begin{array}{l}\text { Faculty, Divisional Academic } \\
\text { Teams for curriculum material } \\
\text { development } \\
\text { IT \& Ed Tech departments for } \\
\text { storage systems }\end{array}$ & $\begin{array}{l}\text { Investigate how other Colleges } \\
\text { are addressing this issue }\end{array}$ \\
\hline $\begin{array}{l}\text { Assure consistency in QA } \\
\text { process implementation across } \\
\text { programs }\end{array}$ & $\begin{array}{l}\text { QA co-ordinator to confirm KC } \\
\text { processes for College in alignment } \\
\text { with System. College } \\
\text { management team to agree on } \\
\text { College processes }\end{array}$ & $\begin{array}{l}\text { Manual of College KCs and } \\
\text { processes established and } \\
\text { stored on shared drive } \\
\text { All programs and courses } \\
\text { incorporate processes into } \\
\text { curriculum delivery }\end{array}$ & $\begin{array}{l}\text { System wide generic processes } \\
\text { Departmental processes being } \\
\text { followed } \\
\text { College established processes }\end{array}$ & College QA coordinator & $\begin{array}{l}\text { Liaise with other Colleges to } \\
\text { investigate what processes } \\
\text { they are using }\end{array}$ \\
\hline $\begin{array}{l}\text { Appoint a dedicated QA } \\
\text { coordinator to manage the QA } \\
\text { project adequately }\end{array}$ & $\begin{array}{l}\text { College Director to allocate } \\
\text { resources needed for physical } \\
\text { office space, IT and human } \\
\text { resources for appointment } \\
\text { Advertisement to be placed }\end{array}$ & $\begin{array}{l}\text { QA co-ordinator appointed } \\
\text { Office space \& equipment } \\
\text { allocated }\end{array}$ & $\begin{array}{l}\text { Generic job description for QA } \\
\text { co-ordinator required }\end{array}$ & Central HR & $\begin{array}{l}\text { Investigate mechanisms in } \\
\text { other colleges } \\
\text { Investigate QA position } \\
\text { applications already on file }\end{array}$ \\
\hline
\end{tabular}




\section{Actions taken}

By the commencement of the next action cycle a QA co-ordinator was appointed so that my role mainly involved mentoring and support within the College. However, I maintained the responsibility of developing College QA processes as a manual, sat on two system-wide process and data committees plus a divisional academic team for program QA. A more structured approach to address the situation commenced, with formal interviews now being arranged with 'problem owners'.

\subsubsection{Cycle 3}

\subsubsection{The Finding Out}

After official approval was given by both the College Director and the RMIT ethics committee to proceed, a more structured approach to the process of social inquiry into the perceived problematical situation commenced in December 2006. Defining action took place in a short period of time due to the work completed over the previous 18 months. Whilst data is pictorially portrayed using rich pictures, interview data was also compiled by issues (App. 7).

\subsection{Rich Picture}

By this stage in Cycle 3, the picture which initially had been drawn at the beginning of the learning cycle had evolved from ongoing meetings, discussions, review of progress through the two less structured cycles and reflections on the dynamics of the situation to involve eight purposeful activities (Figure 24). After analysis and further discussions utilizing information drawn from formal interviews these in turn were refined and prioritized as five transformative statements to reflect five purposeful models of activity. 


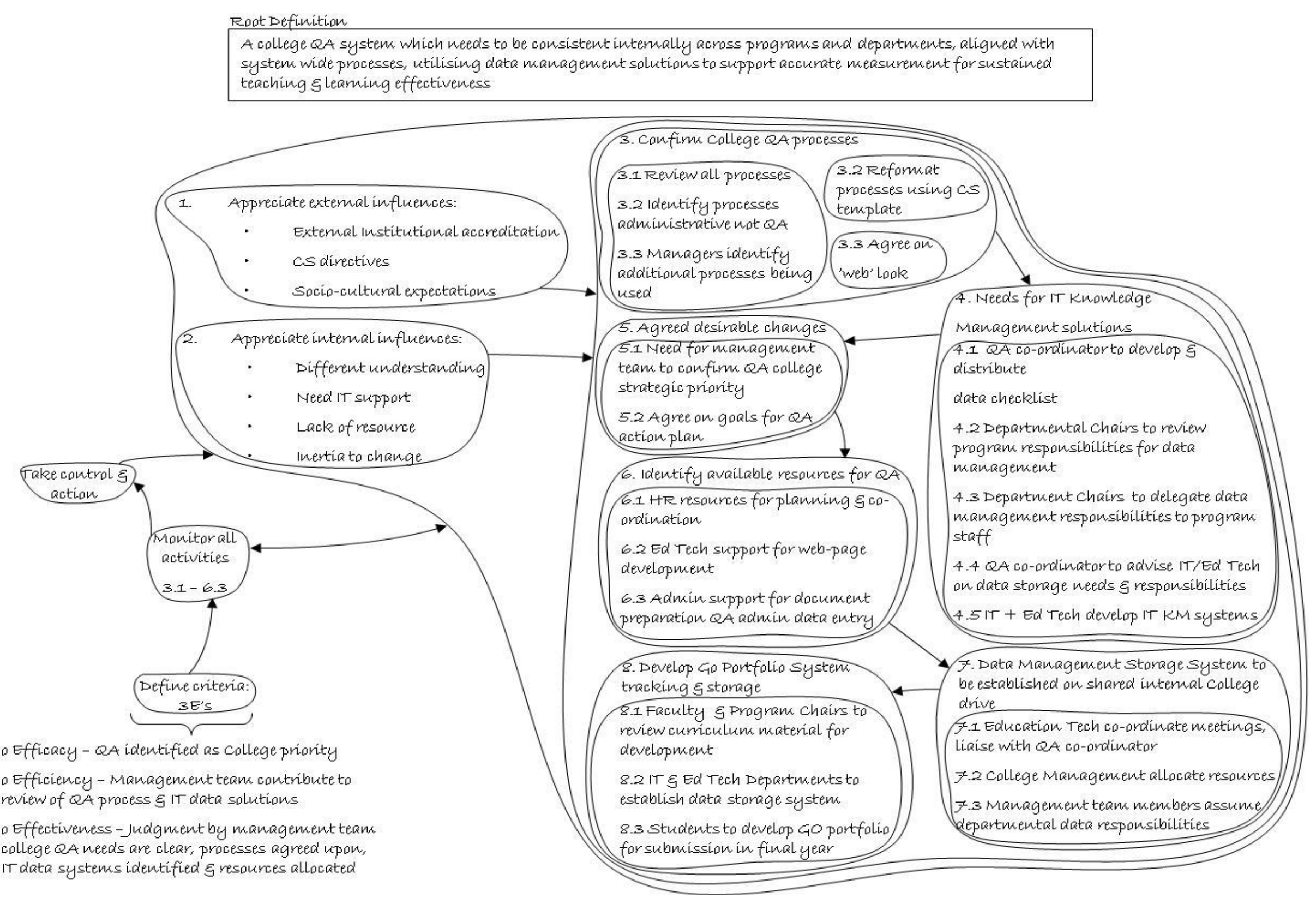




\subsection{Analysis 1}

As noted in Cycle 2, the following stakeholder roles were identified (Figure 25):

Client

Practitioner (problem owner)

Owners
Central Services and policy committee

Me - as Doctoral research student and project manager

College management team
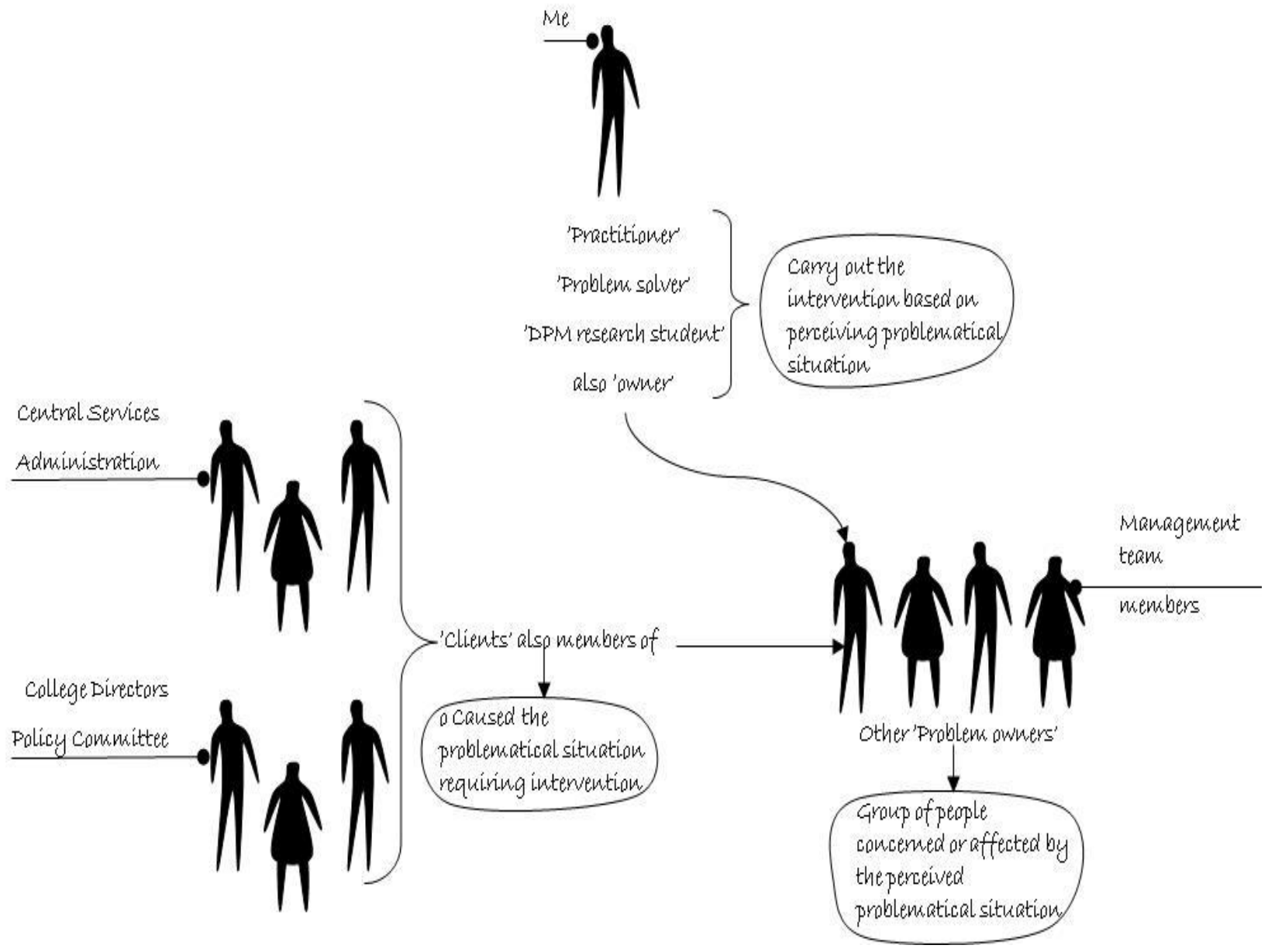

Figure 25 Cycle 3 - Analysis 1 


\subsection{Analysis 2}

The College social system is complex, involving a cultural multiplicity from global sourcing of expatriate employees. Cultural profiling commonly pre-determines a hierarchical system of staffing, such that:

- Faculty are predominantly of UK, Canadian, USA, Australian origin;

- Directors are all male and mainly western, except for one Emirati and one Egyptian;

- Gender and nationalities of management team members vary from college to college according to Director preference, but predominantly are western educated;

- Faculty of Arabic origin (e.g. Lebanese, Iraqi, Syrian, Jordanian, northern Africa) commonly teach lower level certificate and diploma students;

- Academic and Student Services staff are mainly Arabic and Emirati;

- Secretarial staff are mainly of Indian or Arabic nationality, but there has been a mandate to increase the number of national employees in alignment with the country's 'Emiratisation policy';

- Facilities staff are Arabic or Indian;

- Technical services department are Indian;

- Catering and cleaning staff are Philippino or Indian.

There is a preference for global searching for 'new' staff when employees leave rather than promoting existing staff based on experience and qualifications. According to 'problem owners' interviewed for this research, this reality creates an apparent lack of career path and may be seen to contribute to a reasonably high turnover of staff and feelings of being undervalued. "Staff feel threatened, worried about job security, unpredictability in working life; lack of continuity; Expatriates expected to give to the system and country and move on". The majority of Arabic staff share a commonality in Islamic religion and Arabic language despite differences in dialect. The College's organizational functionality is managed primarily by a western expatriate management team but is overlaid by a centralized Arabic system of governance. Language of tuition and communication is English, with faculty being aware that speaking Arabic is not acceptable particularly in the classroom, as this does not assist the Emirati students in furthering their English language skills.

\footnotetext{
${ }^{3}$ Italics comments as recorded in formal interviews
} 
The diverse mix of expatriate staff arrive in the country with their own socio-culturally derived professional norms of behaviour. "People of same background have similar expectations and ways of operating"; "Ways of acting are culturally derived". In turn, the organizational culture had expectations of behavioural norms, the students and families have their understandings of teacher and management roles and the Arabic central administrative system contribute to defining behavioural boundaries. "Some families are very conservative and very globally unaware". The mix of cultures in the work place, in the individual departments and in social circles all vary, creating an imperative to compromise, adapt, be flexible, sensitive and self-aware. It is also to be expected that with such an array of cultures within the one organization that variations in value systems and how people are 'judged' will exist. "Organization and UAE culture so different to the majority of management team members' backgrounds".

Students having received an Arabic junior and secondary schooling are used to didactic teaching styles where the teacher has the 'knowledge' which is to be imparted to the student. "Teachers' skill set is not as relevant at the college". Critical thinking, enquiry and exploration of more than one possible answer therefore are challenging concepts to approach in the College classroom. It is difficult to move beyond the traditional perception of a teacher as being able to provide the one and only 'right' answer. Student self-responsibility is a trait that is promoted by CS administration, filtering to all levels of the education system, but this is at times quite at odds with socio-cultural expectations of some families. Problem owners interviewed in this research project suggested that valuing or liking a teacher may not be judged according to how hard they work or what academic research is involved, but on how many students receive high marks. Some teachers therefore feel pressured to compromise their educational approach - stating that, there is "no research culture, no professional currency"; "People are appointed to fulfil or maintain socio-cultural status quo; people who are different are pushed out".

Therefore the cultural desirability and feasibility of particular actions to make improvement becomes challenging, because although there is a noted "commitment to education" the College's system culture is seen to be "driven by ego-centric, external displays of wealth and importance". There are staff who have a passion for improving systems, but the fear of being too obvious or making comments as being seen as critical rather than proactive can stifle the feasibility of proposed action. "Can't be too vocal, views can't be politicized, no dissent, need to mind your back". A dichotomy therefore exists between wanting or desiring the 'best' education the country can buy and the "institution wanting to maintain ultimate control". Desirable changes over a realistic time period are tempered with an organizational culture which demands immediate, yet frequently changing specificity in results. "Culture of immediacy - everything to be done straight away; causes cynicism in change". Lack of existing processes and no time given to formulate, implement and evaluate 
effectiveness reduces the desirability of particular actions. QA is seen as externally driven rather than as a process that is aligned with improved effectiveness. "External pressures the only incentive to tick boxes"; "no internal desire for improving excellence"; "dichotomy exists - seen as important, but lack of resources allocated".

How and what an individual values as important in their working expatriate life will inevitably affect their perception of what actions are feasible or desirable. If the employees "mind set is 'don't take risks"' or if people feel their "ability to act is hampered/restricted", then these beliefs will underpin acceptable norms of organizational behaviour thus creating impediments to change despite the external imperative to do so (Figure 26). These initial reflections are further explored in the Discussion, Chapter 5.

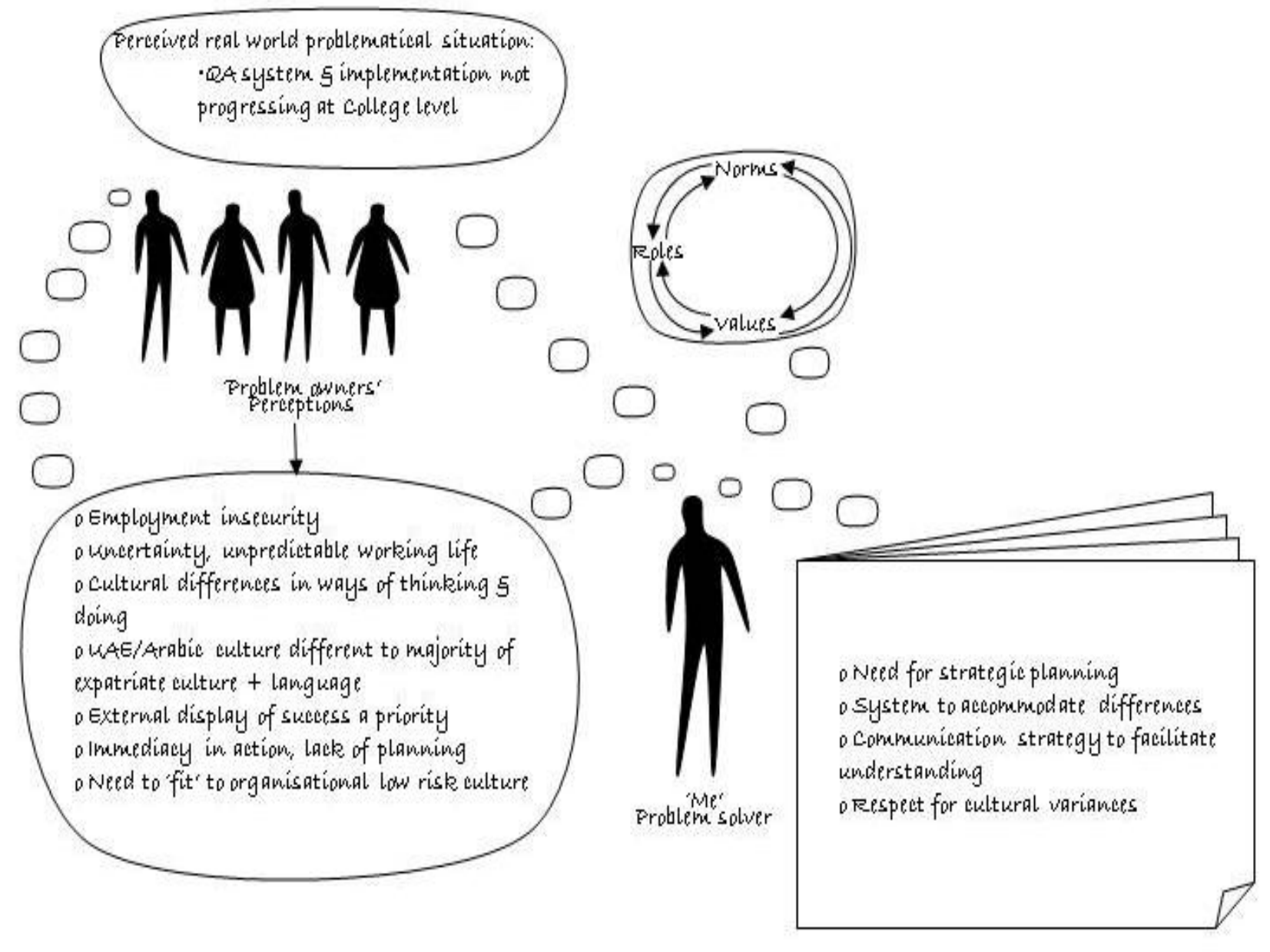

Figure 26 Cycle 3 - Social Analysis 2 


\subsection{Analysis 3}

The politics of the problematical situation determines what action takes place and what is achieved. This further extends upon the feasibility of actions as raised in Analysis 2, and relates to the interplay of power in and between relationships within the organization and socio-cultural context. Power as described in 2.5.3 may be seen as a 'political ontology comprising multiple choices', where a complex social context inevitably entails inequalities in power distribution.

In the context of the problematical situation, power is expressed in various forms. In particular, power as a force of influence has been raised by the 'problem owners' in most of the thematic areas identified (App. 7). Most notably these are found in issues related to 'barriers constraining effectiveness', 'communication', 'socio-cultural' and 'leadership and effectiveness'. The 'power' of the 'client' is a given - that is CS and the policy committee make decisions in relation to moving forward with a particular form of international institutional accreditation and the Colleges are required to action the directives, one of which has been the QA system implementation. This style of operation reflects a traditional bureaucratic organizational structure, where power as a 'commodity' involving Directors and College Management Teams, is instrumentalised through evidence of compliance with centralized directives. "Hierarchical organizational structure; patriarchal attitudes; bureaucratic organizational and operational structure". According to the "problem owners" perceptions of the situation, consequences of non-compliance or lack of progress involved both covert and overt examples of 'punishment and reward' where position promotions, contract terminations and selective inequalities exist between Colleges. "There is a territorial nature of functionality between colleges"; "CS is in control, not always sharing values needed for effectiveness, compromises core business"; "Strategies limited to control destiny from central administration".

Leadership power as personal, political or position, indicates the commodification of power. Ultimate power of authority lies with the national population. There is no legislation which protects the rights of expatriate workers in particular. This absence is seen by 'problem owners' as being abused by those in senior management. There are "bad leaders at high levels"; "behavior is managed by control and fear"; "withholding information, knowledge as power". Personal power is also derived from length of stay in the organization, 'closeness' to influential nationals and a willingness to comply and not challenge authority. "People are appointed/selected to fulfil or maintain sociocultural status quo; people who are different are pushed out"; "It is a system culture which does not abide by regulation". Management is seen as top-down with those in 'powerful' positions being the most influential, although there is a mis-match between authority and responsibility. "Authority is not aligned with responsibility"; "Management culture is patriarchal, authoritarian, hierarchical, 
bureaucratic". Some appointments at the senior level are seen as politically motivated rather than based on academic standing. "Academic skill set of Directors as leaders does not always equate with effective management". These leaders are seen to then put self-survival first, involving the wielding of power over their subordinates in ways which may not be acceptable in their countries of origin particularly those from the 'west'; “...people behave differently to 'back home'; minding their backs; political games; lack of stability and continuity in management".

The multi-layered bureaucratic nature of the organization contributes to complex communication, which is not necessarily uni-directional despite a predominance of top-down directives. That is, the spread of responsibility for communicating appears diffuse, with decisions seemingly made without adequate consultation and communication lacking a transparency. Problem owners reported that this created feelings of insecurity and "powerlessness, giving up trying to be heard; lack of response to queries; uncertainty"; "Communication takes place through inappropriate channels"; "Disconnect between manager and managing group"; "Multiple layers of organization created many opportunities to misinterpret".

Power and control are complex cultural factors which may be related to the maturity of a country and an organization, wherein dimensions of power need to be seen as characteristic of a society's social system rather than as an individual characteristic (Hofstede 2001; Clegg 2006; Dowding 2008). A desire for change and moving forward needs to being tempered with ensuring that the 'change' is needed and managed appropriately. This is compounded by a culture which is striving for the 'best' that money can buy, but not at the expense of comprising socio-cultural norms and values. Thus a reluctance to relinquish 'control' and promote 'risk taking' actions may be seen to limit the pace of effective change. Change is a constant in this organization's functionality. However, a lack of strategic planning and constant changes in the strategic plans themselves, hinder the emergence of an organization where quality and effectiveness correlate. "Constant change can be positive as it provides a flexibility, but it is mainly negative"; "There is a lack of planning and lack of experience as a developing country; the immaturity of the organization reflects an immaturity of the country"; "Society is not as stable, student culture and life is changing, causing an identity crisis"; There is a tendency to "look around the world and grab different things being offered"; "More of a façade, superficiality, preference for things looking good".

Associating a particular style of leadership with the maturity of the organization needs to consider that the organization in which this research project took place is a College in a system established only 20 years ago. Socially accepted norms of leadership and associated instrumentality of power move beyond the confines of an organization per se, to consider the socio-political environment context in which the entity is embedded (Gelfand 2004; Dowding 2008). The maturity of the country 
therefore also needs to be considered with the UAE coming into being only 37 years ago. This, together with traditional purported norms of Arabic leadership behaviour where those in senior positions are expected to be decisive and directive, creates a work environment which may conflict with 'western' expatriate views of management and patterns of communication. "Leadership style changes according to state of organization - 'immature' is in a state of survival, needs more directive leadership; 'intermediate' involves reactive leadership, 'mature' sees more flexible leadership". So whilst the College may be seen as 'immature', problem owners indicated a predominance of staff being more comfortable with organizational operations that involve 'mature functionality'. "More collegiality needed, need to feel valued"; "Leader needs vision and needs to attempt to achieve buyin"; "A good leader benefits from grass-roots feedback". Desirable change may therefore not be feasible, making the process of organizational transformation more complex and challenging (Figure 27).

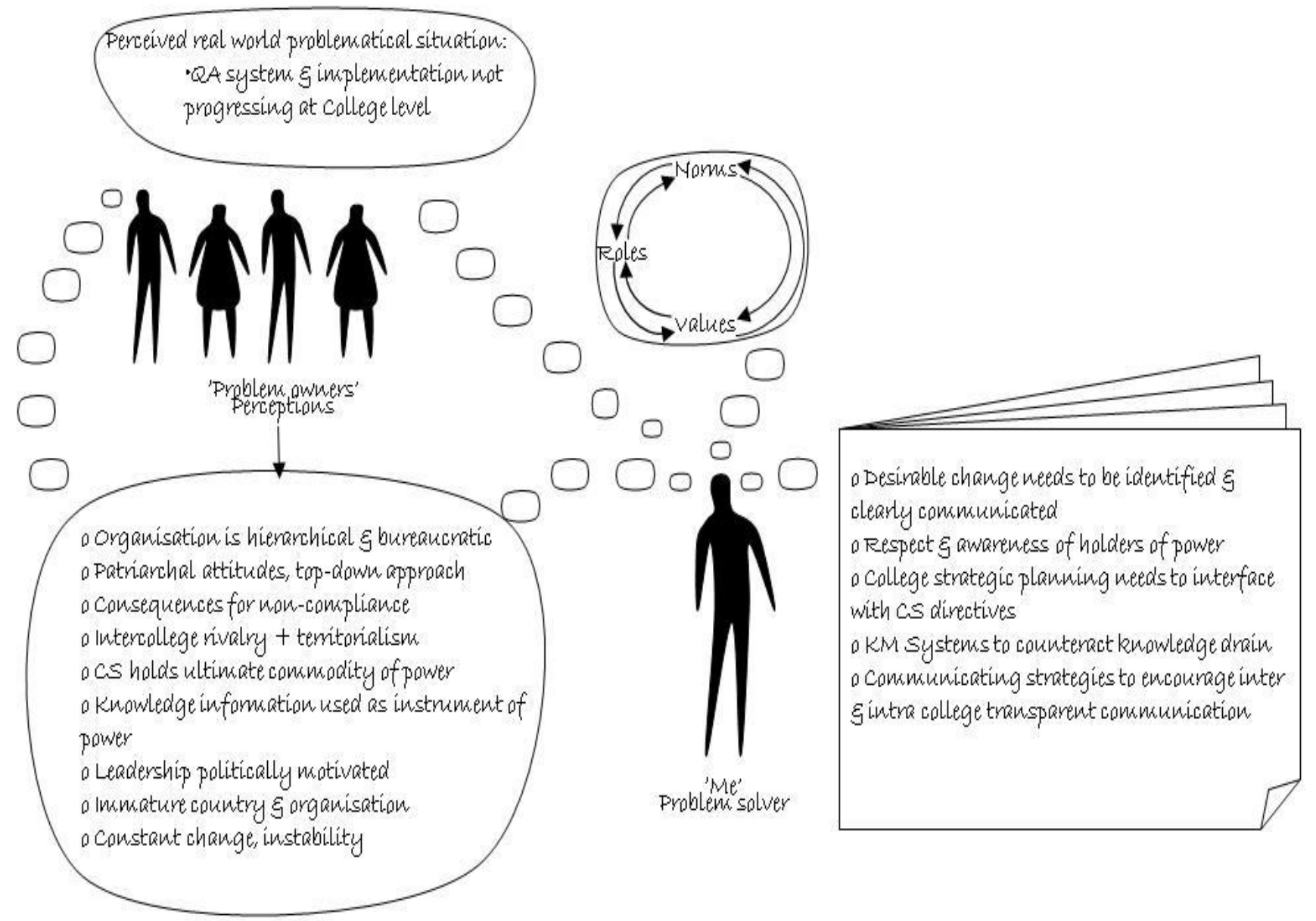

Figure 27 Cycle 3 - Political Analysis 


\subsubsection{Building Purposeful Activity Models \\ 4.2.4.2.1 Expressing the problematic situation - The Generic Model}

The problematic situation from my 'problem owner' reflective view was a lack of progress in College QA system establishment and implementation over a two year period. The reflections and comments included in the previous sections, are subsequent to hearing and considering perceptions of problem owners interviewed formally and the more informal communications through other encounters. Initially a generic model of activity was developed prior to interviewing the fifteen owners of the problem to ascertain their perceptions and to reflect upon their lifeworld constructs of purposeful activity (Figure 28 ).

The Root Definition for this generic model is:

'An Emirati owned educational system staffed by a diverse cultural mix of expatriates and nationals who work together to deliver tertiary educational programs at credible international standards, incorporating ongoing evaluation and review of business practice to improve effectiveness to ensure organizational competitiveness and sustainability'.

To encourage 'problem owners' to express how they perceived the problematical situation, three general open-ended questions were used to initiate discussions: 'What factors do you consider as critical to the effectiveness of an organisation? How do you see Quality Assurance systems correlating with organisational effectiveness? Giving consideration to both of these questions, where did you see the College (in which this research project was being undertaken) as 'fitting' in relation to effectiveness and quality systems?'

In all, five main Root Definitions and activity models were developed from the data and reflective activities, with these correlating with the complete record of ideas from 'owner' interviews as compiled in (App. 7).

In defining and thinking about the concept to be modelled, it is considered that the Primary Task System as reflecting the core purpose of the College is relevant as an overarching statement for each of the transformative statements. This is: "The College is a system that provides tertiary education to national Emirati women to a standard aimed at benchmarking against 'western' best-practice education." However, each of the activity models are considered more 'issue-based' due to being aligned with organizational processes and thinking. 


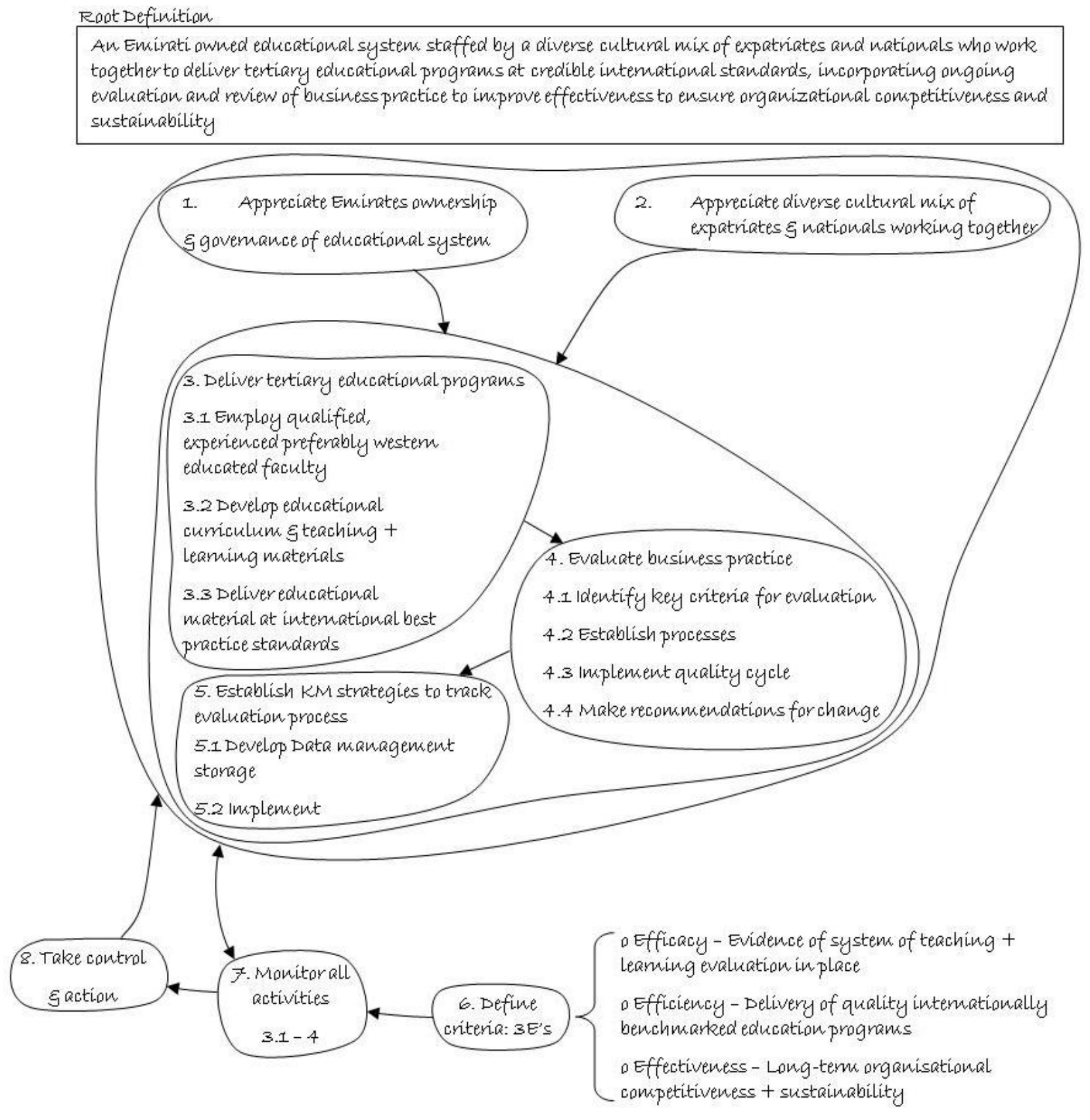

Figure 28 Rich Picture of the Generic Model 
Five key input-output statements as actionable transformations have been identified:

INPUT

1. Need for evaluation of educational delivery

2. QA not aligned with effectiveness

3. Lack of clear communication lines

4. Disconnected knowledge management systems

5. Unplanned, uncoordinated change
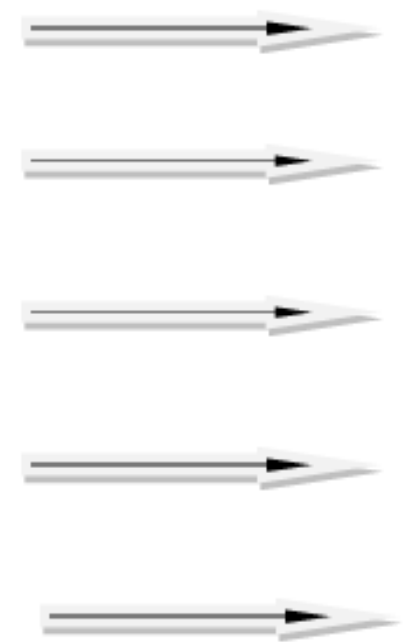

\section{OUTPUT}

Enhanced educational provision

QA values incorporated \&

internalized into all organizational operations

Communication strategies address all stakeholders

Knowledge captured and transferred

Long-term, clarity in planning

In turn, five models were developed using the PQR, Root Definitions, CATWOE and $E_{1}, E_{2}$ and $E_{3}$ stages of in the SSM enquiry process as described in section 3.6 .4 to incorporate these transformations.

The five models of purposeful activity are detailed individually as follows, demonstrating similarities and sharing of elements with the generic model. Models of purposeful activity could have been presented for each 'problem owner', however, in compiling and reflecting upon the data, it was found that a similarity in issues were raised. Therefore, five activity models representing key areas of concern appeared to be a more useful way to accommodate differences to move forward in addressing the problematical situation and the desired output identified. 


\title{
4.2.4.2.2 Purposeful Activity Model One
}

\author{
( ${ }^{*}$ Refer to 3.6.4.3.5 and Glossary for revision of PQR \& CATWOE terminology) \\ P Develop and implement a western derived QA system within the College by \\ Q establishing and confirming QA Key Criteria and College processes \\ $\mathbf{R}$ to provide systems of measurement and evaluation of teaching and learning
}

\section{The Root definition}

An Emirati owned higher education system staffed by a predominantly expatriate faculty and management workforce, which aims to implement a western derived QA system in all Colleges, with management team members being responsible for involving faculty and staff in data collection and analysis to evaluate and make recommendations for improvement to teaching and learning effectiveness for enhanced educational delivery.

\section{CATWOE}

C Students \& Faculty

A Faculty \& Management

T Evaluation of educational delivery to enhance educational provision

W A QA system if connected to educational delivery can improve teaching and learning effectiveness

o College Management Team, Central Administration

E Communication processes, external control systems
$E_{1}$ (Efficacy)
Evidence of data collation and analysis with recommendations for action
$E_{2}$ (Efficiency)
Agreement by management team that College based QA system will have positive effect on teaching/learning activities
$E_{3}$ (Effectiveness)
Judgment by Central Services that the College has an established QA system that provides evidence of ongoing improvement in educational delivery


Reot Definition

An Enirati. owned higher education systen staffed by a predonimantly expatriate faculty and nanagencent workforce, which ains to inplenent a westem derived QA systen in all colleges, with nanagenent tean. nembers being responsible for involving faculty and staff in data collection and analysis to evaluate and make reconnendations for inprovenent to teaching and leaming effectiveness for en hanced educational delivery

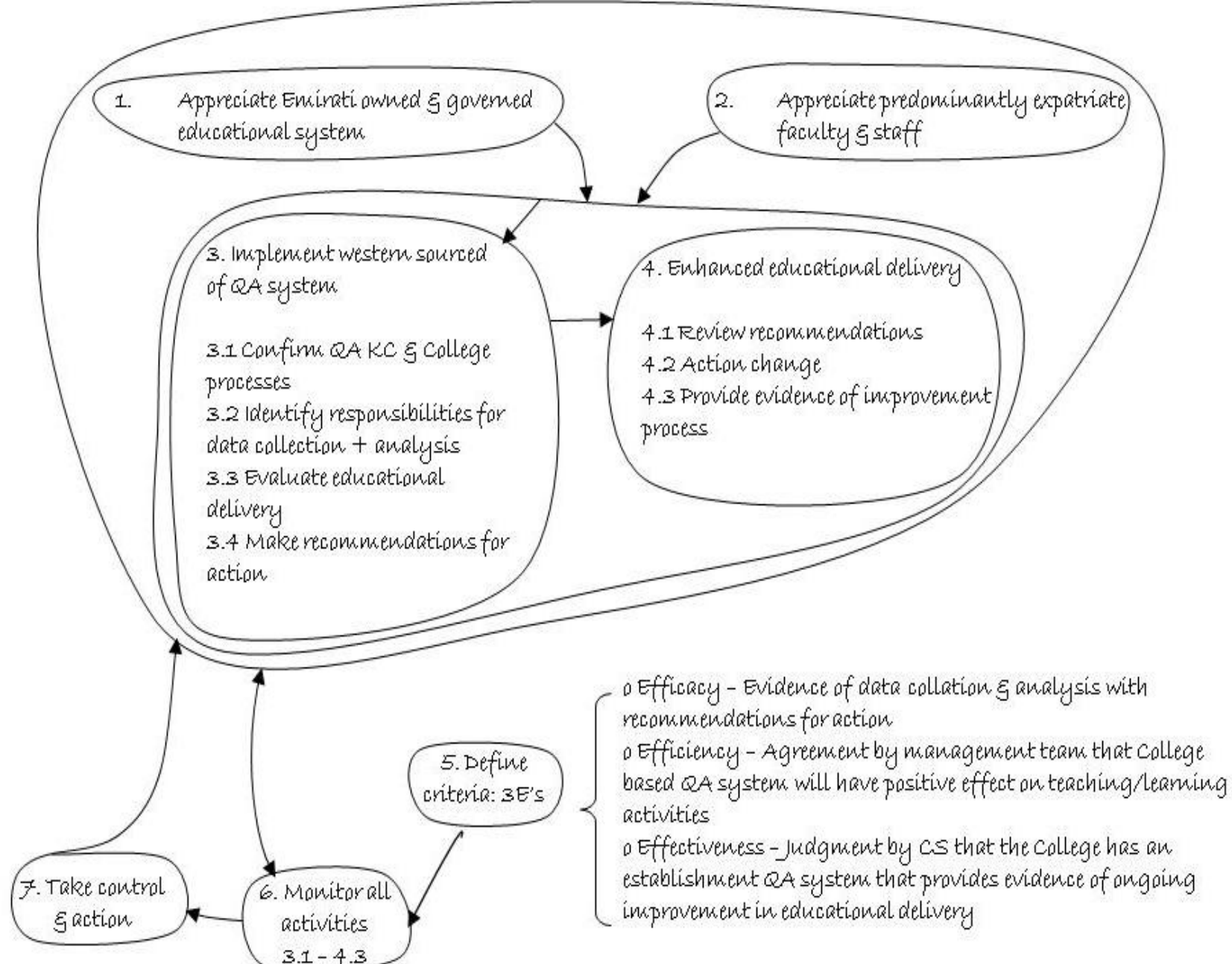

Figure 29 Purposeful Activity Model One 


\subsection{Purposeful Activity Model Two}

P Develop a QA system within the College which involves all stakeholders

Q through allocating sufficient resources

$\mathbf{R} \quad$ to provide ongoing support to implement necessary changes

\section{Root Definition}

An Emirati owned higher education system which involves all stakeholders, students, faculty and college management, in the development and implementation of a QA system through allocating sufficient human and financial resources to embed quality practices for improved organizational effectiveness.

C Students, Faculty, College management

A College management and Faculty

T QA involves incorporating and internalizing QA values into working life

W QA systems need to be embedded to contribute to improved organizational effectiveness

- Central Services, College management

E Resource allocation, specifications for QA, stakeholder buy-in, transient staff, culturally derived disparate understandings
$E_{1}$ (efficacy)
Evidence of QA processes and criteria referenced at the teaching and learning interface
$E_{2}$ (efficiency)
Agreement by all management team members that College Key criteria, consistent processes and system implementation will benefit educational delivery
$\mathbf{E}_{\mathbf{3}}$ (effectiveness) Evidence that all departments are adequately resourced to integrate and utilize QA evaluations in recommending changes to program delivery for improvement 
Ropot Definition

An Encirati. owned highereducation system, which involves all stakeholders, students, faculty and college managenent, in the development and inplencentation, of a QA systen through allocating sufficient human and financial resources to enced quality practices for inproved organizational effectiveness

o Efficacy - Evidence of QA processes and criteria referenced at the teaching and leaming interface

o Efficiency - Agreenent by all managencent tean menbers that college Key criteria, consistent processes and systenk inplencentation will benefit educational delivery

- Effectiveness - Evidence that all departucents are adequately integrated and resourced to utilize QA evaluations in reconnuending changes to program delivery for inprovencent
2. Appreciate nultiple stakeholders

o Students $\varepsilon$ fancilies

- Faculty $\xi_{\text {staff }}$

- collegenanagenent

csadninistration

agreencent on collegeprocesses

3.2 Departument chairs

inplencent data collection, entry ganalysis

3.3 Departukent chairs nake reconsunendations for action

3.4 QA co-ordinatorprovides support g guidance

5. QA systen inclenentation

5.1 Managenent tean to initiate QA processes in their departunents

5.2 QA co-ordinator to provide support $\varepsilon$ information to faculty $\varepsilon^{2}$ Departne ent chair 5.3 QA co-ordinator to facilitate collegewide consistency

4.I Managencent tean SKPPort QA initiatives

4.2 Director conknits financial

resources to QA system

inclencented based on

reconkmendations

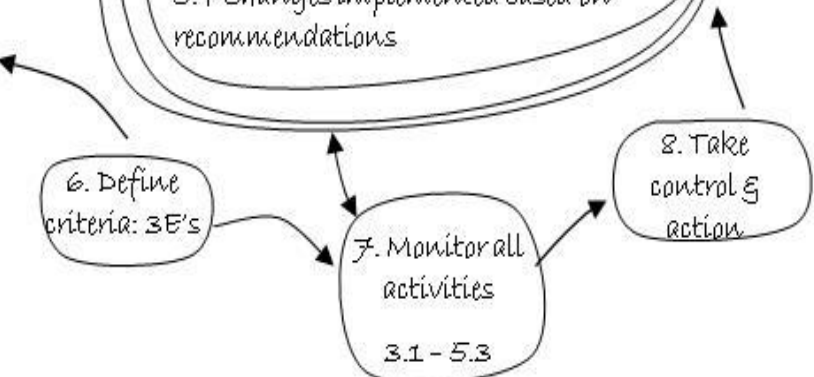

Figure 30 Purposeful Activity Model Two 


\subsection{Purposeful Activity Model Three}

P Develop a clear College communication strategy involving all stakeholders

Q by allocating key responsibilities and promoting opportunities for dialogue

$\mathbf{R}$ to accommodate different ways of communicating and lessen feelings of uncertainty

\section{Root Definition}

An Emirati owned higher education system which has multiple organizational layers and discursive communication patterns needing clear strategy development to improve interpersonal connectivity and lessen feelings of uncertainty to improve productivity through a sense of involvement and being informed.

C Faculty, College management

A College Management

T Communication strategy to connect all layers of the organization to reduce uncertainty

W Clear lines of communication involving all stakeholders can improve productivity through all being informed

- College Management, Faculty, Central Services

E Multiple layers of responsibilities within the College, dual/multiple staff and management roles, varied communication styles, transient staffing, interpretative differences, Central Services external directives, leadership style differences

$\mathbf{E}_{\mathbf{1}}$ (Efficacy) Evidence of new lines of communication between management and faculty

$\mathbf{E}_{\mathbf{2}}$ (Efficiency) Agreement by management team members that there are benefits in taking the time to develop a communication strategy

$\mathbf{E}_{\mathbf{3}}$ (Effectiveness) Judgment by faculty and management team members that they feel informed and aware of what is happening in the College 
An Encinati. owned highereducation systen which has nultiple organisational Layers and discursive connknnication pattems needing clear strategy developnent to inprove interpersonal connectivity and lessen feelings of uncertainty to improve productivity through sense of involvencent and being infomaed

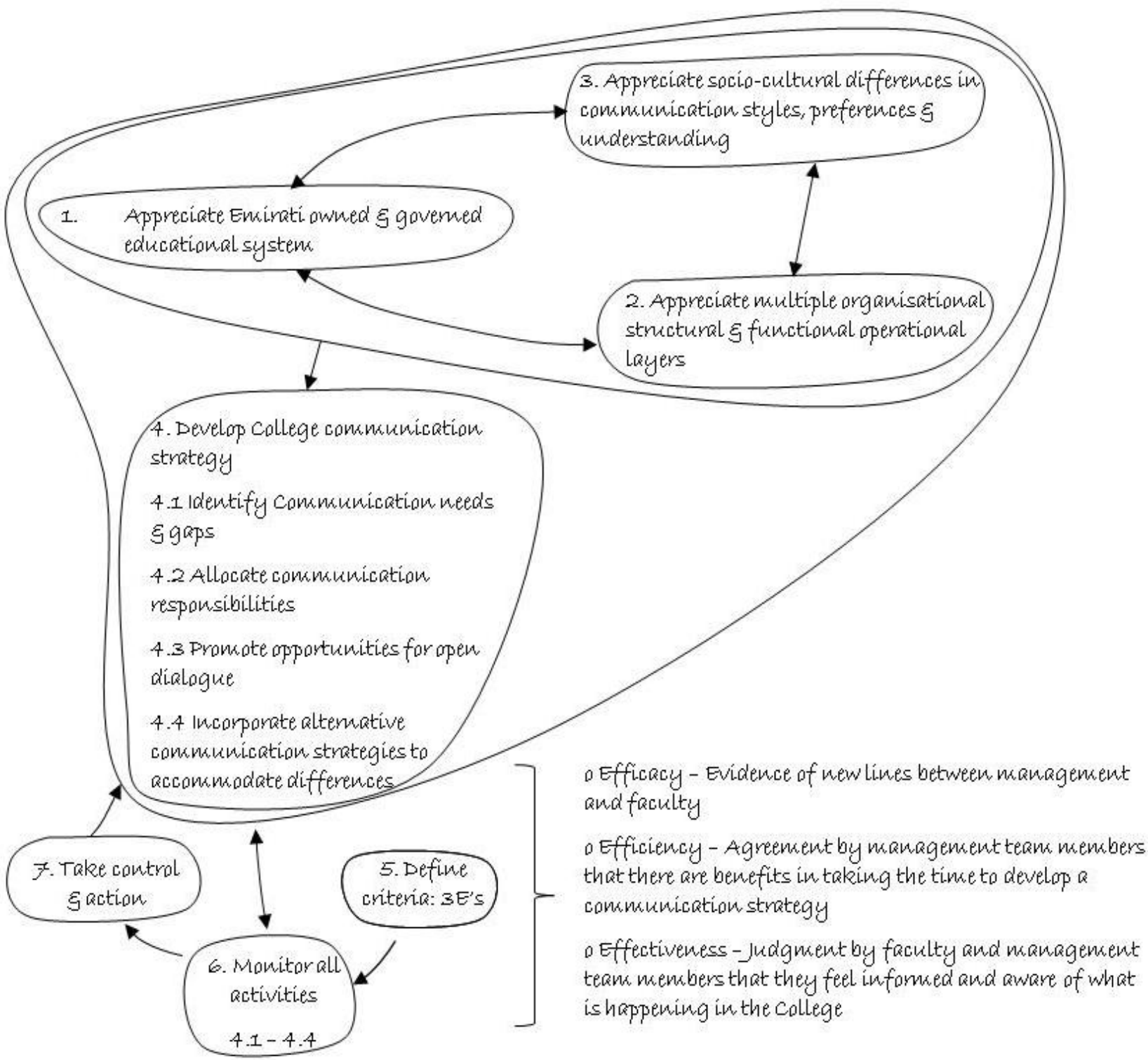

Figure 31 Purposeful Activity Model Three 


\subsection{Purposeful Activity Model Four}
$\mathbf{P} \quad$ Develop policies and take strategic action
Q to guide development of data management collection and storage solutions
$\mathbf{R}$ to facilitate the capture and transfer of knowledge for improved effectiveness

\section{Root Definition}

An Emirati owned higher education system with a transient and diverse socio-cultural staff mix, working towards policy development and strategic action to guide the establishment of data management collection and storage solutions through adequate resource allocation, to assist with the capture and transfer of knowledge for improved effectiveness.

C Faculty, College management
A College management
T Policies and systems established for improved knowledge connectivity
W Knowledge management systems can assist with capture and transfer of knowledge for improved effectiveness
- Central Services, College management, other Colleges
E IT support staff and resources, IT systems accommodating prioritized needs, Central Services directing IT solutions, transient staff
$\mathbf{E}_{1}$ (Efficacy) Evidence of policies and strategies supporting the establishment of College knowledge management systems
$\mathbf{E}_{\mathbf{2}}$ (Efficiency) Agreement by Director and management team that IT data base support warrants prioritized resource allocation for improved organizational effectiveness
$\mathbf{E}_{\mathbf{3}}$ (Effectiveness) Judgment by faculty and management team that IT solutions are user- friendly and are facilitating the storage and transfer of knowledge effectively


Root Definition

An Eninati. owned higher education systen, with a transient and diverse socio-cultural staff nix, working towands policy development and strategic action to guide the establishnent of data managencent collection ana storage solutions through adequate resource allocation, to assist with the capture and transfer of knowledge for inproved effectiveness

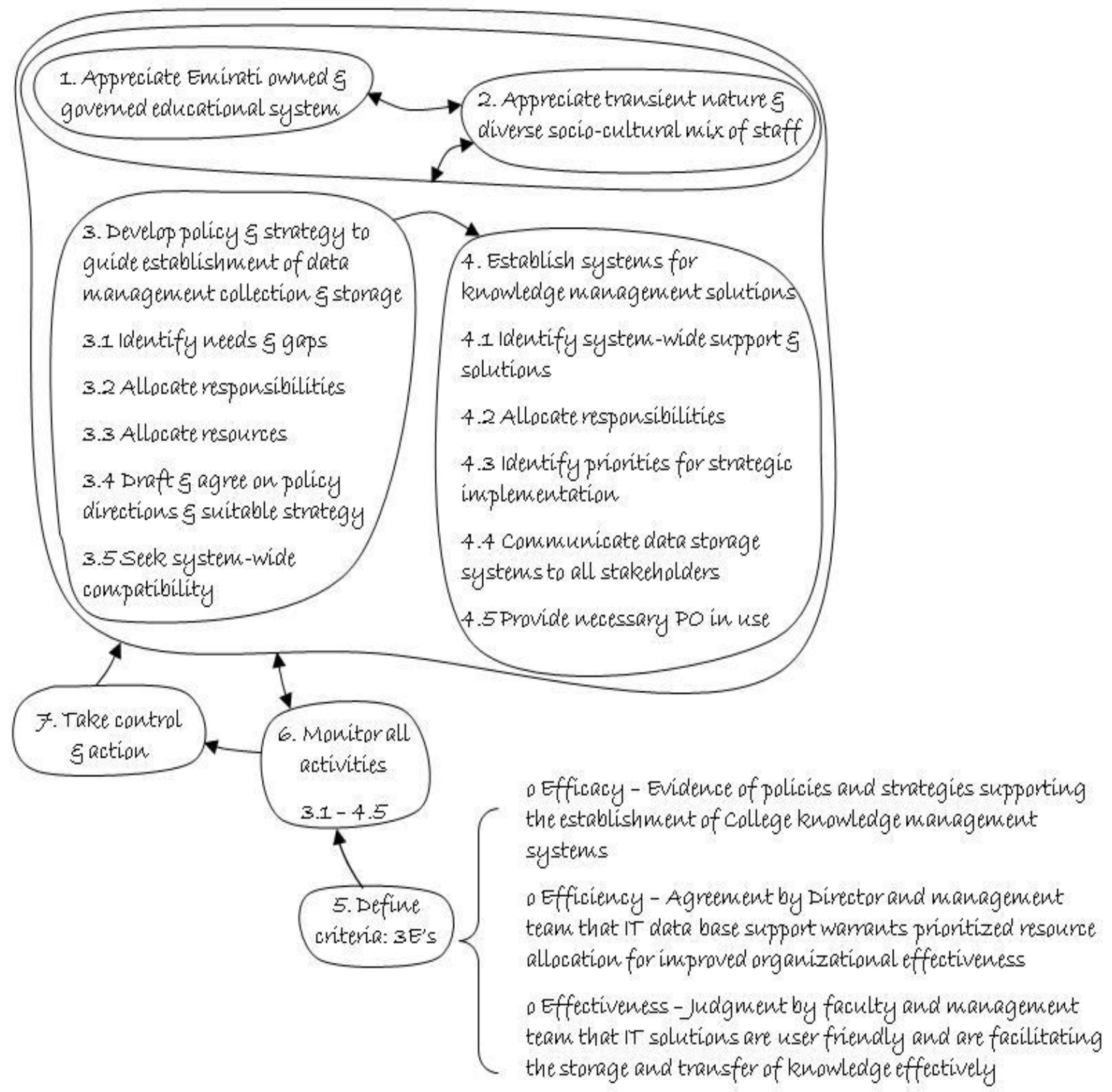

Figure 32 Purposeful Activity Model Four 


\subsection{Purposeful Activity Model Five}

P Develop a College 5-Year strategic plan involving all stakeholders

Q by consulting widely and providing opportunity for students, families, faculty and management

$\mathbf{R}$ to contribute to the development and communication of planned outcomes and strategies for the development of the College

\section{Root Definition}

An Emirati owned higher education system that involves the Emirati student and family population, faculty, management team members and all other staff, in the development and communication of a 5-year strategic plan to guide and co-ordinate purposeful organizational change that can weather but accommodate changes in priorities, staffing and external directives.

C Students, families, faculty, College management

A College management

T Organisational change is co-ordinated and strategically planned

W Long-term strategic planning can clarify direction and give a sense of purpose for change initiatives

- Central Services, College management

E Socio-cultural differences in identifying priorities and planning for organizational future, inertia to change, Central services influence on college directions, fluid nature of changing priorities, unpredictable funding, transient nature of staffing and College management

\footnotetext{
$E_{1}$ (Efficacy)

Evidence of a 5-year strategic plan

$E_{2}$ (Efficiency)

Support by all stakeholders for the plan and agreement on the positive effect future planning has on organization sustainability

$\mathbf{E}_{\mathbf{3}}$ (Effectiveness) Judgment that the strategic planning activity involving all stakeholders is a worthwhile activity; publication of strategic plan; all departmental plans draw upon the College strategic plan; support from Central services for the strategically planned outcomes
} 
Ropot Definition

An Encinati owned highereducation system that involves the Encinati student and family population, faculty managenent team nembers and all other staff, in the development and conm nuication of a 5 -year strategic plan to guide and co-ordinate purposeful organisational changethat can weather but acconno odate changes in prionities, staffing and extemal directives

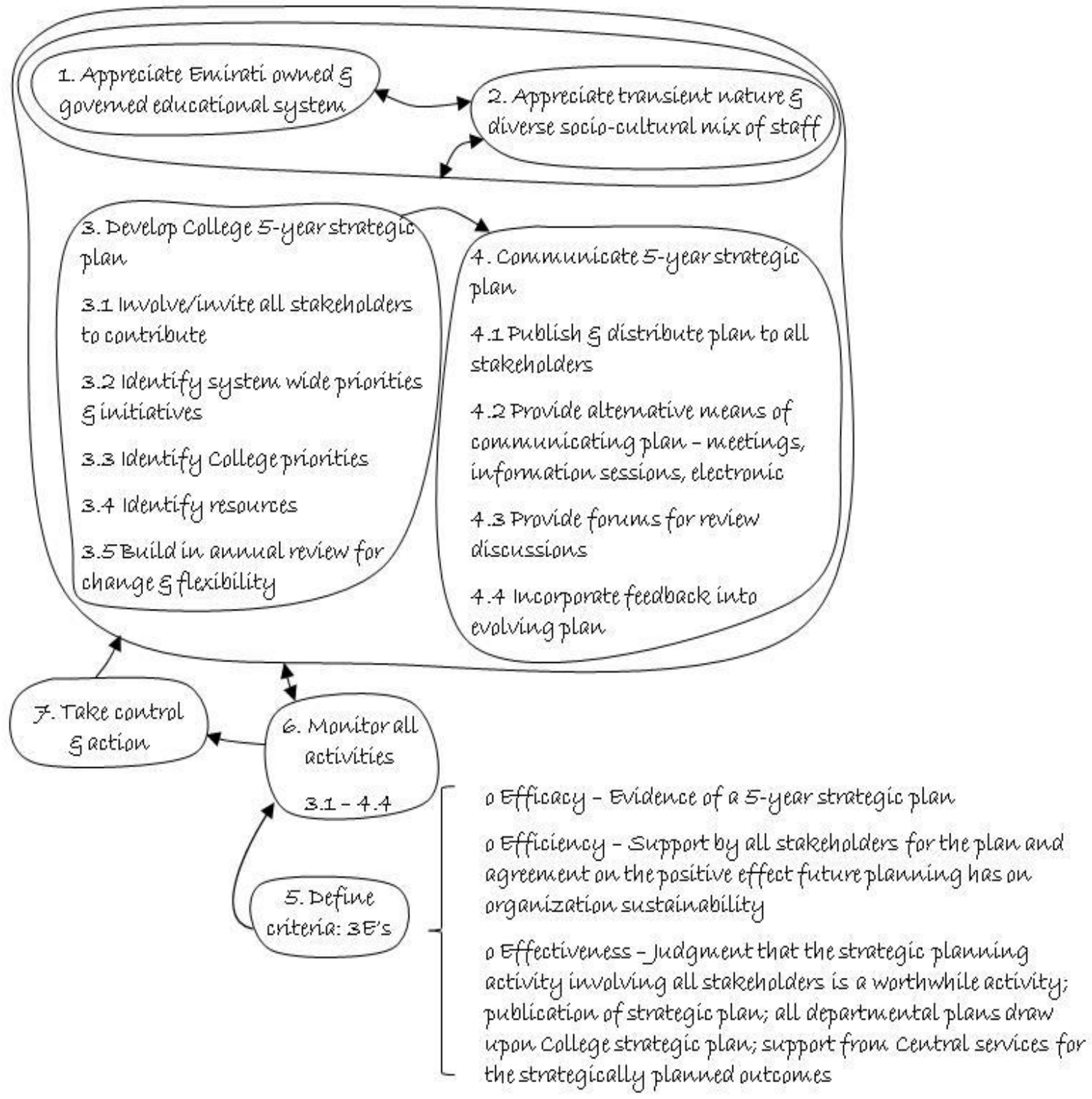

Figure 33 Purposeful Activity Model Five 


\subsubsection{Discussing the situation to generate ideas}

The five main identified areas as represented by different root definitions and transformational processes guided ongoing discussions with owners of the problem using 'debate to seek or create accommodations to enable action to improve the problematical situation' (Checkland and Winter 2006).

The five purposeful activity models compiled from reflections upon dialogue in formal and informal interviews were then compared with the initial generic model, with information transferred into a matrix for conceptual purposes to set the models against perceived realities (see Table 8). Whilst there were five main activity models identified, these in turn comprised other models of activity as potential transformational processes. An overall picture comprising these is presented at the beginning of Cycle 4.

\subsubsection{Defining desirable and feasible change}

The five activity models as identified by the owners of the problem involved:

i. implementing a QA system to enhance educational provision

ii. incorporating QA values into all aspects of organizational operations

iii. developing communication strategies to involve all stakeholders

iv. developing KM solutions for knowledge capture and transfer

v. strategic planning involving 5-year articulated outcomes

These were all considered 'desirable' changes, with the 'feasibility' of each being affected by the social and political perceived realities inherent to the organization and context. To define a way forward for improving upon the problematical situations a focus on the 'how' (as reflected in the ' $Q$ ' of the PQR formula) to make the actions possible was considered, incorporating information into a table format for clarity (Table 9). 
Table 8 Matrix comparing activity models

\begin{tabular}{|c|c|c|c|c|c|}
\hline & Activity Models & Exist or not in real situation? & How is it done? & How will it be judged? & Comments \\
\hline General & $\begin{array}{l}\text { Emirati owned higher } \\
\text { education system delivering } \\
\text { western standard programs } \\
\text { that require evaluation \& } \\
\text { KM solutions to promote } \\
\text { effectiveness }\end{array}$ & $\begin{array}{l}\text { Rudimentary evaluation processes, } \\
\text { not as established systems. Lacking } \\
\text { KM solutions particularly to } \\
\text { accommodate for transient } \\
\text { population }\end{array}$ & $\begin{array}{l}\text { Currently QA is by educational program to } \\
\text { meet international institutional accreditation } \\
\text { requirements. Centralised system of data } \\
\text { storage initiated not supported by all Colleges. } \\
\text { No defined College KM solution }\end{array}$ & $\begin{array}{l}\text { Annual program audited reports, } \\
\text { external and internal review. }\end{array}$ & $\begin{array}{l}\text { Programs inconsistently } \\
\text { seeking international } \\
\text { accreditation running parallel } \\
\text { to institutional accreditation, \& } \\
\text { QA processes }\end{array}$ \\
\hline 1 & $\begin{array}{l}\text { Enhance educational } \\
\text { delivery through developing } \\
\text { \&implementing a QA system }\end{array}$ & $\begin{array}{l}\text { College Key criteria and processes } \\
\text { under development. Buy-in by all } \\
\text { stakeholders lacking }\end{array}$ & $\begin{array}{l}\text { College management team needs to review } \\
\text { system-wide specifications \& confirm College } \\
\text { direction }\end{array}$ & $\begin{array}{l}\text { Evidence of measurement and } \\
\text { evaluation of teaching \& learning }\end{array}$ & $\begin{array}{l}\text { External control limits } \\
\text { autonomy. Resources need to } \\
\text { be allocated for QA system } \\
\text { implementation. }\end{array}$ \\
\hline 2 & $\begin{array}{l}\text { Incorporate QA values into } \\
\text { all elements of } \\
\text { organizational operations }\end{array}$ & $\begin{array}{l}\text { QA exists as a disjointed overlay to } \\
\text { program delivery. QA disconnected } \\
\text { from organizational effectiveness. QA } \\
\text { lacks clarity, is confusing \& creates } \\
\text { additional administrative load. }\end{array}$ & $\begin{array}{l}\text { QA system needs to be developed subsequent } \\
\text { to agreement at management level. QA } \\
\text { currently not being resourced adequately. }\end{array}$ & $\begin{array}{l}\text { Documented evidence of QA } \\
\text { implementation, adequate } \\
\text { resources allocated, } \\
\text { recommendations actioned and } \\
\text { implemented, teaching \& learning } \\
\text { delivery evaluated }\end{array}$ & $\begin{array}{l}\text { Current QA loops not closed. } \\
\text { Lack of system-level } \\
\text { agreements. System does note } \\
\text { capture teacher \& learning } \\
\text { effectiveness. QA controls } \\
\text { externally derived. }\end{array}$ \\
\hline 3 & $\begin{array}{l}\text { Develop a communication } \\
\text { strategy which involves all } \\
\text { stakeholders }\end{array}$ & $\begin{array}{l}\text { Transparency in communication } \\
\text { lacking. Top-down approach, but } \\
\text { inconsistent, unpatterned \& } \\
\text { disconnected, creating feelings of } \\
\text { being ill-informed. No clear strategy. }\end{array}$ & $\begin{array}{l}\text { Multiple layers of communication with } \\
\text { duplication, overlap, misinterpretations \& } \\
\text { gaps. Information overload rather than } \\
\text { selective. }\end{array}$ & $\begin{array}{l}\text { Stakeholders feel informed, clear } \\
\text { strategies identified \& followed. } \\
\text { Constructive feedback systems } \\
\text { operational. Time for reflection \& } \\
\text { dialogue. }\end{array}$ & $\begin{array}{l}\text { Communication fear-driven. } \\
\text { Not all stakeholders involved } \\
\text { nor informed. Inappropriate } \\
\text { channels of communication }\end{array}$ \\
\hline 4 & $\begin{array}{l}\text { Establish KM solutions which } \\
\text { support the capture and } \\
\text { transfer of organizational } \\
\text { knowledge }\end{array}$ & $\begin{array}{l}\text { KM piecemeal approach, information } \\
\text { transfer disjointed, knowledge leaves } \\
\text { the College when transient } \\
\text { expatriates leave. Lack of policy to } \\
\text { guide knowledge capture \& transfer }\end{array}$ & $\begin{array}{l}\text { State of the art IT systems, but lacking in } \\
\text { software and storage solutions. Lack of policy } \\
\text { and strategy to guide action. Knowledge } \\
\text { storage associated with individuals rather than } \\
\text { College. }\end{array}$ & $\begin{array}{l}\text { Developed KM policies. } \\
\text { Established data storage systems } \\
\text { supported by relevant software } \\
\text { applications. Faculty and staff feel } \\
\text { informed \& aware of accessing } \\
\text { relevant data. }\end{array}$ & $\begin{array}{l}\text { Clarification between Central \& } \\
\text { College based systems of KM } \\
\text { needed. Management team } \\
\text { needs to support solutions. } \\
\text { Resources need allocating. }\end{array}$ \\
\hline 5 & $\begin{array}{l}\text { Develop five-year College } \\
\text { strategic plan to reduce } \\
\text { uncertainty and improve } \\
\text { clarity in organisational } \\
\text { direction }\end{array}$ & $\begin{array}{l}\text { Five-year College strategic plan } \\
\text { drafted, with annual review and } \\
\text { changes. Not all stakeholders } \\
\text { involved, creating feelings of plan } \\
\text { being superimposed. CS directives } \\
\text { instigated ad hoc changes. Lack of } \\
\text { clarity in college strategic directions. }\end{array}$ & $\begin{array}{l}\text { Director drafts plan, circulates to management } \\
\text { team for input \& review. Resources allocated } \\
\text { according to CS \& Director perceived priorities. } \\
\text { IT \& more vocal managers perceived as } \\
\text { successful in receiving resources \& support for } \\
\text { initiatives. Elements of plan disconnected from } \\
\text { day-to-day realities }\end{array}$ & $\begin{array}{l}\text { 5-year strategic plan developed } \\
\text { involving all stakeholders. Plan is } \\
\text { communicated via various } \\
\text { alternative means. All } \\
\text { stakeholders feel planning is a } \\
\text { worthwhile process. }\end{array}$ & $\begin{array}{l}\text { Planning process lacks long- } \\
\text { term approach. Changes often } \\
\text { externally driven. Vision needs } \\
\text { to be agreed and articulated. } \\
\text { More guidelines \& directions } \\
\text { needed. }\end{array}$ \\
\hline
\end{tabular}


Table 9 Linking desirability and feasibility with action

\begin{tabular}{|c|c|c|c|}
\hline 'How' & Desirable? & Feasible? & Possible Action \\
\hline $\begin{array}{l}\text { Establish and confirm QA Key } \\
\text { Criteria and College Processes }\end{array}$ & Yes & Yes & $\begin{array}{l}\text { QA coordinator identify } \\
\text { system-wide KCs and } \\
\text { processes. Incorporate unique } \\
\text { College processes, compile } \\
\text { process manual, circulate for } \\
\text { comment, review, then use }\end{array}$ \\
\hline $\begin{array}{l}\text { Allocate sufficient resources } \\
\text { to QA development and } \\
\text { implementation }\end{array}$ & Yes & Yes & $\begin{array}{l}\text { Director to source central } \\
\text { funds and explore College } \\
\text { funds for resourcing. } \\
\text { Management team members } \\
\text { to identify specific resourcing } \\
\text { needs. Dedicated QA } \\
\text { coordinator to be appointed. }\end{array}$ \\
\hline $\begin{array}{l}\text { Allocate key communication } \\
\text { responsibilities and promote } \\
\text { increased opportunity for } \\
\text { internal college dialogue }\end{array}$ & Yes & Yes & $\begin{array}{l}\text { Management team identify } \\
\text { concerns and gaps in lines of } \\
\text { communication. } \\
\text { Subcommittee of } \\
\text { management team develop } \\
\text { strategy, allocating } \\
\text { responsibilities, promoting } \\
\text { opportunities for dialogue, } \\
\text { and providing alternative } \\
\text { means of interpersonal } \\
\text { communication }\end{array}$ \\
\hline $\begin{array}{l}\text { Develop data management } \\
\text { collection and storage } \\
\text { solutions for improved } \\
\text { knowledge management } \\
\text { systems }\end{array}$ & Yes & $\begin{array}{l}\text { Changes in CS priorities } \\
\text { impacts on College plans and } \\
\text { directions. Preference for } \\
\text { short-term planning. Changes } \\
\text { in staff impact on any long- } \\
\text { term plans. Funding changes } \\
\text { annually. Student families } \\
\text { difficult to involve due to } \\
\text { language and cultural factors. }\end{array}$ & $\begin{array}{l}\text { Director and management } \\
\text { team identify College } \\
\text { priorities and compare with } \\
\text { CS plan. Plan should dovetail } \\
\text { into system-wide priorities. All } \\
\text { stakeholders to be invited for } \\
\text { involvement. Discussion } \\
\text { forums and alternative means } \\
\text { of communication to be } \\
\text { incorporated into consultative } \\
\text { process. }\end{array}$ \\
\hline
\end{tabular}


Defined actions as defined were all desirable but some were less feasible than others. This was perhaps a result of working with managers who were familiar within the College system and who were used to compromising and identifying the realities in context. Political and social analyses also contributed to reflections about the situation in determining limitations in desirable or feasible change. One of the major challenges which emerged from the confirmation of these five activity models particularly given their interdependent nature, was the feasibility in co-ordinating the action necessary to implement all five models concurrently.

However, at the point of preparing for presentation of defined actions to the management team in the new semester commencing January 2007, my employment contract was ceased suddenly with 48 hour notice. No reason or explanation was given. There had been no performance issues and all reviews were excellent. The contract termination was issued by the new College Director who had assumed the role a few months prior at the time when the previous College Director was moved to another campus. In seeking justification for the termination, I suggested to the new Director that employment contracts were usually ceased for professional or personal reasons, and I was unaware of any professional basis for ceasing my employ so suddenly. His response was a shrug of the shoulders, stating that it must be personal, thus adding insight to the organisational culture itself. Despite rapid and unexplained contract terminations taking place throughout the organisation over the years in which I had been working at the College, faculty and students with whom I had been working still shared my surprise as to the unexpectedness. Within two weeks another manager was given notice, which was followed by action that resulted in a number of management team members not having their contracts renewed and others deciding to resign from the organisation. By the end of a 12 month period there were very few of the original 23 management team members still employed at the College.

The addressing of the content of the problematical situation therefore finished with defining action, however, another cycle involving further reflections and purposeful planning was instigated, involving email communication and small group meetings with those managers still willing to be involved. 


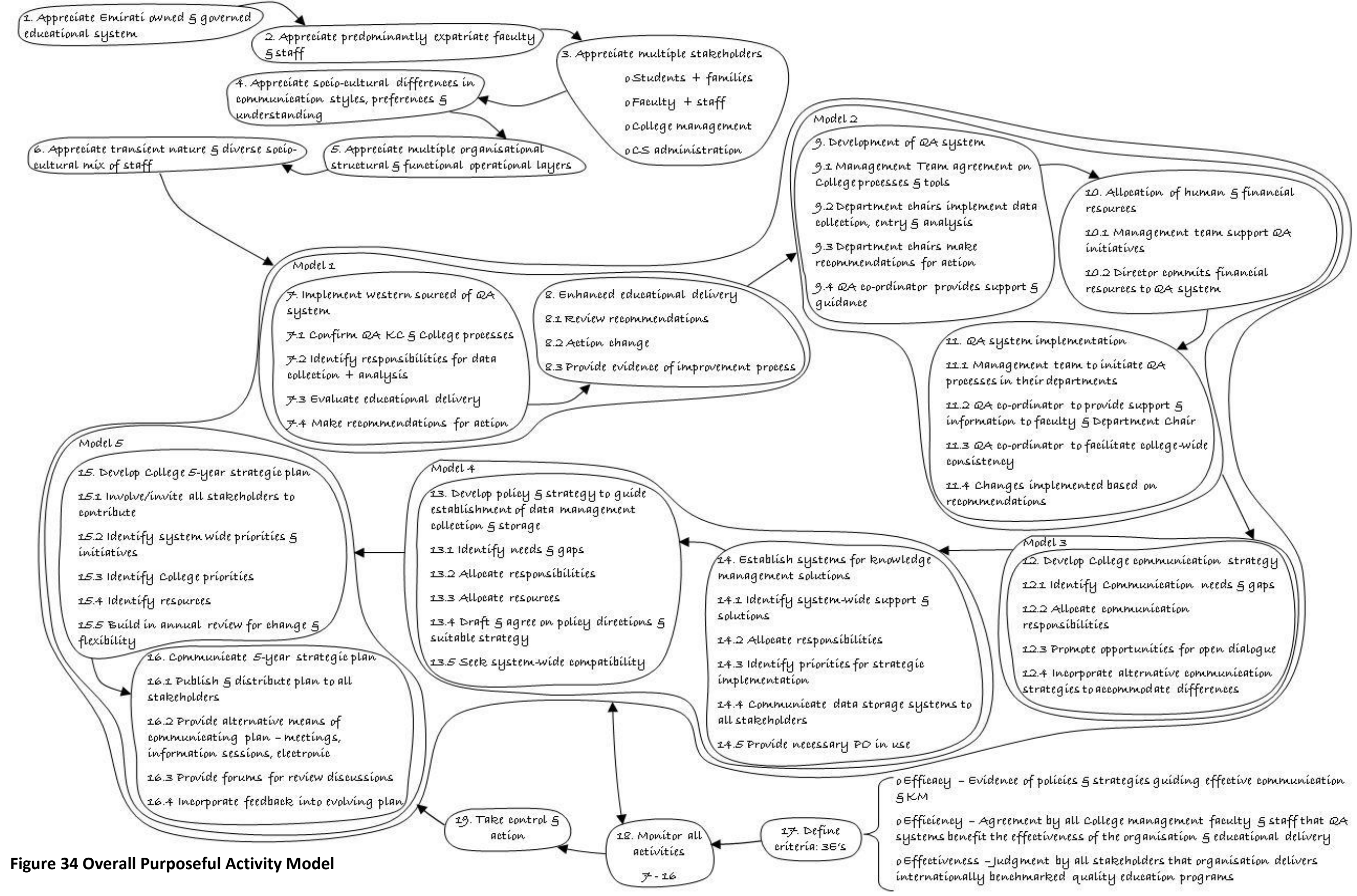




\subsubsection{Cycle 4}

The rich picture of the problematical situation at the point of defining action had thus evolved as represented above in Figure 34 as the new purposeful activity model.

As a result of reflecting upon the interview data, meeting minutes, informal discussion notes and the overall purposeful activity model developed throughout the cycles of action to this point, three questions seemed to surface as problem areas warranting further exploration or clarification.

These were:

i) Do you think there is any particular style of leadership which is more effective and why?

ii) What strategies or approaches could facilitate improved communication when working with people from culturally diverse backgrounds?

iii) What strategies could be implemented to manage learning for organizational change given the socio-cultural environment?

These were emailed to all managers who had indicated willingness to be involved in the project, with a summary of responses included in Appendix 8.

The answers to these questions structured the discussions about desirable and feasible change to improve the situation as presented in Table 10. Actions for improvement and the feasibility of interventions, noted the limitations and constraints the socio-cultural context has on effective change, with these factors being explored and discussed in the Chapter 5 . 
Table 10 Factors affecting organisational effectiveness

\begin{tabular}{|c|c|c|c|}
\hline 'How' & Desirable? & Feasible? & Possible Action \\
\hline $\begin{array}{l}\text { Organizational effectiveness } \\
\text { correlates with leadership } \\
\text { style }\end{array}$ & Yes & $\begin{array}{l}\text { Leaders are appointed to } \\
\text { maintain a degree of status } \\
\text { quo. Preferred leadership style } \\
\text { is strongly influenced by the } \\
\text { Arabic socio-cultural context. } \\
\text { Leaders need to listen but be } \\
\text { seen to be decisive. }\end{array}$ & $\begin{array}{l}\text { Action by faculty and staff in } \\
\text { relation to leadership selection is } \\
\text { limited. Therefore staff may } \\
\text { need to compromise own } \\
\text { expectations and preferred style, } \\
\text { whilst recognizing leadership } \\
\text { style which is respected and } \\
\text { expected in an Arabic context. } \\
\text { Need to acknowledge that } \\
\text { respect and 'saving face' } \\
\text { underlies most communication } \\
\text { and that perceived image is very } \\
\text { important. Managers should be } \\
\text { consultative but decisive. } \\
\text { Decisions and actions need to be } \\
\text { taken giving consideration to the } \\
\text { system of governance managing } \\
\text { the College. }\end{array}$ \\
\hline $\begin{array}{l}\text { Communication strategies } \\
\text { need to accommodate for } \\
\text { culturally diverse teams of } \\
\text { employees }\end{array}$ & Yes & $\begin{array}{l}\text { Yes, but overlay of disparate } \\
\text { cultural factors adds to social } \\
\text { complexity arising from } \\
\text { personality differences which } \\
\text { exist in most work settings }\end{array}$ & $\begin{array}{l}\text { Professional development, } \\
\text { education programs and } \\
\text { awareness of cultural differences } \\
\text { and ways of communication } \\
\text { needed. Leaders, which may be } \\
\text { department or project managers, } \\
\text { need to recognize and } \\
\text { acknowledge differences 'up } \\
\text { front'; Leaders need to create } \\
\text { the environment; Leaders need } \\
\text { to pick the right blend of people; } \\
\text { Managers need to be publicly } \\
\text { clear, transparent, directive, } \\
\text { establish an environment that } \\
\text { promotes trust and make people } \\
\text { feel safer and able to take risks. } \\
\text { Leaders need to 'protect' their } \\
\text { workers from external threats }\end{array}$ \\
\hline $\begin{array}{l}\text { Organizational change } \\
\text { strategies need to } \\
\text { accommodate for the socio- } \\
\text { cultural environment }\end{array}$ & Yes & $\begin{array}{l}\text { Perhaps - but quite } \\
\text { challenging. Low risk taking } \\
\text { culture is a disincentive to } \\
\text { suggest or implement radical } \\
\text { change. Change tends to be } \\
\text { reactionary rather than } \\
\text { proactive or strategically } \\
\text { planned. Organizational } \\
\text { environment is unpredictable, } \\
\text { and although this can be } \\
\text { rewarding and challenging, too } \\
\text { much uncertainty and change } \\
\text { for the sake of change, doesn't } \\
\text { work }\end{array}$ & $\begin{array}{l}\text { Organizational change needs to } \\
\text { be strategically planned. } \\
\text { Adequate resourcing is needed } \\
\text { to support people to do their } \\
\text { best so the organization can } \\
\text { move forward. Need for change } \\
\text { should be communicated for } \\
\text { people to feel informed and see } \\
\text { the purpose in action. }\end{array}$ \\
\hline
\end{tabular}




\subsection{Summary and conclusion}

The research described in this dissertation used a Soft Systems Methodological approach to enquire into a messy problematical situation arising from attempts to implement a Quality Assurance system in a higher education College in the UAE. Project implementation involved four iterative learning action cycles, with the first two and the fourth focusing on the planning the approach and intellectual process (SSMp) whilst the third cycle worked through addressing the content of the problem (SSMp) in an attempt to bring about improvements to the situation. The QA implementation and research project spanned a time period from August 2005 to June 2007.

The first cycle of action involved finding out about the problem through my experience and perceptions as 'problem solver' in and through reflections on interactions with 'problem owners' and other stakeholders through both formal and informal interactions. A number of issues were identified as warranting action as noted in Cycle 1 Action matrix (Table 6), with these realities confirming for me that there was a situation that was perceived as problematical and worthy of research.

Cycle 2 move beyond 'finding out' about the problem, to involve analysis and reflection upon both the social and political context of the situation. There appeared unrest and confusion about the purpose or direction of the QA system being imposed upon the College from Central Services administration. Models of action began to evolve from management meeting action items and reflection upon other interactions. It seemed apparent that agreement on action was missing, yet similarities in frustration about lack of progress and confusion of requirements in relation to QA were shared. Perceptions about causes and the realities of the problematical situation varied, but a number of agreed re-prioritised actions were defined as presented in Cycle 2 Action Matrix (Table 7). Throughout the exploration, rich pictures were drawn to portray the situation, with these evolving and emerging over time. By the beginning Cycle 3 a detailed rich picture involving eight purposeful activities had developed (see Figure 24). These purposeful activities included, confirming College QA processes, need for IT Knowledge management solutions, agreement on QA as strategic priority, resource allocation requirements, data management solutions and graduate outcome portfolio strategies.

Cycle 3 involved a more structured approach to the enquiry, incorporating 'problem owners' perceptions from the formal interviews together with reflection upon notes made from informal interactions, into the social and political analyses. What became apparent throughout this cycle was the degree of agreement regarding the desirability of change, but a perceived complexity in the feasibility of required action. Issues arising from the organization being embedded in an Arabic 
cultural context and the power relationships inherent and interconnected in all aspects of working life, arose from what was becoming a complex model building process.

Five actionable transformational statements emerged as preceding the development of five purposeful models of activity. These are represented diagrammatically in Figs 29-33, with information being transferred into a matrix to compare them with the generic model developed (Table 8). The five main actions identified as potentially contributing to improving the situation involved, enhancing educational delivery through use of a QA system, incorporating QA values into all elements of organizational operations, developing a communication strategy to involve all College stakeholders, establishing KM solutions to support the capture and transfer of organizational knowledge particularly given the transient nature of expatriate employees, and the imperative to develop a five-year College strategic plan to reduce uncertainty in organizational direction.

Desirability and feasibility of 'how' these actions may be implemented were discussed with a number of owners of the problem, with outcomes presented in Table 9 'linking desirability and feasibility with action'. Actions were therefore defined, but limited opportunity to implement them arose due to the sudden cessation of my employment contract.

A fourth cycle of action continued outside the organizational context, bringing together six sociocontextual concerns that were identified as needing to be appreciated in actioning five overall models of activity involving ten purposeful activity processes (Figure 34). When comparing these models with worldviews, three areas relating to leadership, communication and organizational change strategies arose as being pivotal to effective transformational processes. These desirability and feasibility of actions for organisational effectiveness, given the social and political context is summarized in Table 10, revealing again, a complexity which needs to be factored in to project management in contexts exhibiting similar human complexity. The issues which have emerged throughout this exploratory process are now discussed in the next Chapter. 


\section{Chapter 5 Discussion and conclusions}

\subsection{The significance of the project context}

\subsubsection{Introduction}

The following chapter draws upon issues and factors arising from the process of enquiry into a perceived project management problematical situation as presented and explored in the previous chapters. The primary aim of the research described in this dissertation has been to explore the significance of the socio-cultural context, socio-technical infrastructure and human capital elements considered relevant to the management of projects for effecting organizational change. Noting the socio-cultural disparities of the employees in the project organisation the second aim has been to explore the feasibility in developing project strategy to accommodate for such difference to facilitate the enacted cognition necessary for learning and organisational resilience. The research was initiated subsequent to encountering problems when implementing a QA system within a higher education institute in the Middle East. SSM as selected and used as the process of enquiry, has been instrumental in facilitating the emergence and identification of critical issues pertaining to project complexity originating from human and environment interconnections.

This research aimed to move beyond the structure-agency divide which tends to dominate the positivist models of project management research and practice (Cooke-Davies, Cicmil et al. 2007), to reveal the social nature of project complexity where inextricable human-environment interconnections need to be recognised as the project actuality. A conceptual framework to underpin thinking in relation to such contextual realities in today's project environments has been defined with enactivism as an 'anti-dualist' theory of cognition guiding ontological and epistemological assumptions that support the view of project praxis as social process (Winter, Smith et al. 2006). Acknowledging a structural interrelatedness between actors as embedded in context helps shift the project management focus from linear, instrumental models of project implementation to one where complexity, social interaction and indeterminate, yet patterned experience, dominate project actuality (Söderlund 2004a; Cooke-Davies, Cicmil et al. 2007). Viewing the project 'entity' as structurally open and interconnected within an organisational and environmental context, facilitates appreciation of the cognitive enactment which emerges in adaptive systems whose resilience is dependent upon balancing an ontological state of being with incremental change typifying an ontology of becoming (Baerveldt and Verheggen 1999; Lineham and Kavanagh 2006; Proulx 2008). 
Project practice involves mutual reciprocal human relationships where learning is the constant emergent product resulting from assimilated knowledge created from inseparable agent and structure interconnections. The dynamic nature of these human interconnections in project praxis are made more complex when socio-cultural disparities between actors exist, bringing relational inequalities to the forefront of project actuality. The 'emergent realities of project praxis in socially complex project environments' demand appreciation and understanding if successful project processes and outcomes are to be evidenced. Discussions that focus on teamwork, collegiality and consensus as being pivotal to successful project practice will benefit further from recognition of the competing narratives and conflicting discourses that call for strategic management in the facilitation of organisational learning (Boddy and Paton 2004; Bresnen 2006). Complex 'games' involving the instrumentality of power, issues of autonomy and control, together with whose interests are best being served, are more the norm in today's world of business (Dowding 2008). As workplaces become increasingly 'international' from global sourcing of expertise and ease of employment relocation, social enmeshment of disparate mixes of human elements in project environments are now creating a project complexity of a 'human kind'. The research in this dissertation aimed to address such realities, supporting the demand for new understandings and approaches to project management to facilitate emergent models for superior practice (Cooke-Davies and Arzymanow 2003).

The ensuing discussion draws upon the six system areas of appreciation which emerged through the exploration as presented in Figure 34 in the Overall Purposeful Activity Model. These factors as identified by problem owners in this research are considered relevant to project praxis within other socio-cultural contexts where project complexity as social process is the real project actuality. Through developing purposeful activity models, this research saw the emergence in appreciation of the following significant factors:

- The organisation as an Emirati owned and governed educational institute;

- The organisation being predominantly staffed by expatriates;

- A multiplicity of stakeholders involved - including students, families, faculty, staff, college management and a centrally located external administration;

- Differences in stakeholder socio-cultural backgrounds, influencing communication patterns, preferences and understandings;

- Multiple organisational structural and functional operational layers;

- The transient nature and diverse socio-cultural mix of expatriate staff. 
The discussion chapter is structured into five sections to incorporate these factors, to fulfil the aim of exploring the socio-cultural elements perceived as significant to managing projects, together with ascertaining the feasibility of defining project strategy to guide the project manager's response given the interrelated nature of multiple contextual factors. More specifically, the first section (5.1) discusses the significance of the project context through exploring the influence of the socio-cultural environment given the nature of the country and the organisation in which the research was undertaken. Related elements of language, communication and cognition as significant to project praxis are then given consideration, followed by an exploration into the perceived barriers, constraints and complex power games that may hinder the enactment of cognition and the leadership necessary to circumnavigate the flux of environmental factors. The second section (5.2) proceeds to explore projects as social process, noting the complexity arising from differences created from human plurality and the challenges faced in defining project strategy to guide the project manager's response given the emergent realities of project praxis. The third section (5.3) notes the limitations of this research, a fourth section (5.4) makes a number of recommendations for further research and the fifth section (5.5) concludes the dissertation.

\subsubsection{The influence of the socio-cultural environment}

\section{The Country}

As introduced in section 3.2 and expanded upon in section 4.1.2, the UAE is a Middle Eastern country that came into being in its present political form in 1971 as Britain withdrew from the region and the federation of seven of the Trucial States took place. The Arabic language and Islamic religion define the identity of the Gulf countries to which the UAE belongs (Loosemore and AI MusImani 1999). The UAE is governed by the Emirati Royal family with leadership succession of Sheikhs being based on inheritance and ruling national family appointments. Islamic principles of jurisprudence underpin Shari'a Law, which acts as the binding legal framework operating within the country. The heavy reliance on an expatriate workforce for infrastructure development over the past three decades has seen the overall population of the UAE grow to almost 5 million, of which only $20 \%$ are nationals, and the overall population estimated as increasing annually at $3.833 \%$ (CIA 2008). Such population demographics where the minority in numbers 'rules' a population majority comprising a sociocultural mix of expatriates, creates a multiplicity of social inequalities overlaid by a socio-political dichotomy created by an Arabic system of governance holding ultimate power of authority over the higher proportion of non-Emirati residents. The wealth of the nation of which nearly $40 \%$ of the GDP is drawn from current and future estimated oil and gas reserves (CIA 2008), has provided a high standard of living for those fortunate enough to have access to salaries which at a minimum, 
compare favourably with the western world. The dependency on expatriates in most industry sectors, together with the inevitable finite nature of natural resources and the absence of natural water supplies, are critical challenges that the country needs to address now and into the future. The UAE today has seen extensive growth and change from its origins as a Bedouin society whose traditional livelihood in the past was dependent primarily upon fishing, pearling and date farming.

Given the realities of the UAE's rapid and dramatic infrastructure changes over the last few decades, together with the diverse socio-cultural mix of expatriates, a non-egalitarian political system and huge population growth, it would seem inevitable that tensions from resultant social inequalities and different cultural understandings of systems of accountability will present (Gelfand 2004). The research findings in this dissertation support this supposition, revealing the multiple layers of system governance, power games and challenges created by socio-cultural diversity in project practice.

\section{Maturity}

Inadequate or inappropriate response to such complexity was perceived by problem owners as related to the maturity of the country and in turn the organisation. As noted earlier in this dissertation, institutional and national maturity have been identified as being associated with the degree of nation-state permeability to international influence (Hofstede 2001; Cooke-Davies and Arzymanow 2003). As enmeshment of cultures becomes increasingly evident from the ease of international travel and the sourcing of employees on a global scale, workforce dynamics are shifting to models that are non-static, mobile and humanly plural (Held, McGrew et al. 1999). The interplay and influence of diverse social mixes of human capital arising from these complexes, will inevitably influence both organisational and national culture, particularly given the emergent cognitive phenomena that arise through human and environmental interconnections. Associating the maturity of organisations with project practice in different industries (Cooke-Davies and Arzymanow 2003) or drawing upon different organizational maturity models which have been developed in attempts to measure organisational response capabilities, may assist in visualising factors which can affect project practice in socially complex environments. The 'Novice to Expert' model of skill acquisition of Dreyfus (1986) and Benner (1984), the 'ACapability Maturity Model Integration' (CMMI) or the Portfolio, Programme and Project Management Maturity Model (P3M3) (APM 2008-9) are all examples of models that identify characteristics typifying levels of process maturity or competency development. Levels of maturity in the CMMI and P3M3 models range from an awareness of process with ad hoc action through different levels of process existence to the upper level of maturity as defined by processes being optimized, organizationally embedded and integral to proactive and

\footnotetext{
${ }^{4}$ Developed by Carnegie Mellon's Software Engineering Institute SEI. (2009). "Capability Maturity Model Integrated." Retrieved 20th January, 2008, from http://www.sei.cmu.edu/cmmi/general/index.html.
} 
continuous process improvement. The skill development or competency model of maturity (Benner 1984 ) is more commonly referred to in relation to individual maturity of skills, but is equally applicable to organisational maturity for its levels of consciousness and cognitive awareness. Range of performance encompass novice behaviour typical of limited experience through five levels to expert behaviour as the most mature where breadth of experience underpins intuitive action.

Problem owners interviewed for this research raised the issue of UAE and organisational maturity as key factors impacting on effectiveness, with comments suggesting that the country and in turn the project organisation, appear to display characteristics typical of 'novice' or inexperienced behaviour where 'rule-governed' inflexible approaches to contextual situations are common. As a country whose federated existence spans just over three decades however, it is probably not unexpected to witness ad hoc spontaneous change typical of less mature nations where awareness of process and only limited repeatable processes exist. Behaviours which exhibit a rigid adherence to rules, a lack of discretionary judgement and the development of guidelines for action that mirror other countries (Benner 1984; Dreyfus 1986; APM 2008-9; SEI 2009), are characteristics associated not only with politically younger countries of lesser maturity, but societies with a collectivist nature, such as identified for nations in the Arabic region (Kabasakal and Bodur 2002). Risk aversion, and the avoidance of uncertainty are both characteristics of immature institutions and collectivist nations, where both limited experience and the protection of kinship influence behaviour to limit change and maintain status quo through systems of social control (Hofstede 1980; Gelfand 2004). As raised by problem owners in this research, awareness of the need for process, yet limited evidence of repeatable processes and erratic, reactive approaches to development with limited planning, all typify an organisation or country which is still maturing.

The issue of country maturity and the correlation with organisational and industry maturity raised by problem owners in this research is supported in the literature (Rogers 1995; Carlopio 1998; CookeDavies and Arzymanow 2003; Walker, Maqsood et al. 2005). Yet the restrictions in length of this dissertation, limits the breadth of discussions in relation to maturity models possible. However, the significance of the interplay and embedded nature of power as constraining and enabling action in organisational functionality as reflective of system maturity is worthy of note, particularly in relation to the complex game forms being acted out in the research project organisation. Barriers identified as constraining organisational effectiveness and issues related to lack of individual and organisational autonomy in the research context, reflect a socio-cultural environment that is grappling with the constancy of rapid change, where ${ }^{5}$ 'knee-jerk' reactions and 'crisis-management' dominate daily business practice. The 'lack of time to plan', the 'lack of strategic planning and lack of policy and long-term directions' where the organisation, as is the country, appears to be functioning at a novice

\footnotetext{
${ }^{5}$ Italics as per interview data recorded in Appendix 7
} 
level of maturity, results in 'organizations always in a state of response and reaction' with 'constant change' and 'ill-informed decision making'.

Socio-political complexity and change

The social complexity resulting from the culturally plural expatriate population existing throughout the country, create challenges in gaining some semblance of assimilated understanding as employees of western, middle eastern and Asian origin interrelate within working environments governed by an Arabic Islamic socio-political system. The dichotomies that exist when striving for western standard business practices in a world dominated by traditional Arabic value systems create tensions which arise from the presence of an epistemological pluralism where difference is the socially complex reality (Healy 2003; Cooke-Davies, Cicmil et al. 2007). Problem owners interviewed for this research, noted the challenges that constancy of 'ongoing, unplanned change' has, particularly in reducing the 'the time for reflection', with these comments relating to both working and social life. The selective sourcing of expatriates by country according to profession creates social divides that are compounded by financial inequalities and employment opportunities. Short-term work contracts are the norm, perpetuating a sense of transience in living, with insecurities compounded for those with lesser opportunities in their home country. A lack of labour or anti-discrimination legislation compounds the employment uncertainties and instabilities as identified by problem owners, with sudden unexplained contract terminations adding to the 'fear-driven communication' and feelings of being 'threatened, worried about job security and the unpredictability in working life' dominating ways of operating.

As noted by Heard-Bey (2004) "...the UAE is gradually being swept into a whirl of strategic, political, economic, social and cultural changes..." (p.xxviii). It is therefore not surprising that the issue of national capacity to be competitively involved in a sustainable manner with international business is of concern, particularly by those in positions of authority. In 2007 for example, at the launch of the 'Dubai Strategic Plan 2015', His Highness Shaikh Mohammad Bin Rashid Al Maktoum, Vice President and Prime Minister of UAE and Ruler of Dubai spoke of the growth of the nation being dependent upon continued social development and the involvement of nationals at all level of the workforce. He emphasized the importance of the knowledge era and the need to enhance human capital through improving educational standards and access to the same. As quoted in Rahman (2007), Shaikh Mohamed stated: "It is common knowledge that it is far easier to build financial capital than it is to build intellectual, psychological and moral capital. Building a road, or a bridge, may take a year or two, but building a person takes a lifetime". Thus the recognition of the importance of human capital development, albeit the focus on the Emirati population, has been evident at the most senior and influential levels of the UAE government with the establishment of quality educational institutions and the development of research based initiatives all relevant worthy actions for long- 
term nation-state sustainability. The challenges faced in attempts to achieve the nation's optimal capacity however, is the mismatch between desire for continuous improvement necessitating proactive responses, and the reality that the maturity and age in years of the UAE results in business processes still evolving and being of limited repeatability.

Recognition of the need to optimise the wealth of emergent knowledge being enacted within the diverse expatriate workforce, as potential to facilitate evolving maturity is not voiced publicly. Problem owners identified the lack of knowledge management strategy as contributing to the limitations of the organisation effectively moving forward. It would seem that there is an urgency to not only invest in new educational and business initiatives as identified by Shaikh Mohammad for the sole benefit of the national population, but to explore strategic ways of managing and sustaining the growth of assimilated cognition from the expatriate-national population interconnections. Through recognising the value and strategically managing the emergent cognition from the transient multicultural expatriate workforce, organisational and nation-state social resilience is a more likely scenario. "Knowledge is in the space of emergence where knower and known meet and co-influence each other..." (Proulx 2008 p.23) This, however, necessitates a shift in strategic thinking by those with the socio-political power to do so, to see value, rather than threat in difference, to recognise that relationships of interobjectivity can bring forth or transform a world. As suggested by Foucault (1977) power can produce knowledge, but if the power as embedded in the structure of contexts serves to maintain control of the individual as inextricably linked in context, then the limitations on personal autonomy and action will hinder not only the enactment of cognition, but the aims of action in the first instance (Dowding 2008). Project strategy to accommodate for difference at the project or organisational level needs to take into account the nature of project praxis as embedded in wider socio-political environments. The challenge is to accommodate for such contexts and ascertain effective responses when connections today involve a globalised world that is "...complex, elusive, ephemeral and unpredictable..." (Law and Urry 2004 p.404).

\section{Socio-cultural change}

In comparing the Bedouin UAE society of 30 or 40 years ago with today, there is however evidence that the culture of UAE has not been static, rather incremental change resulting from the alterations in financial wealth of the country and the increasing permeability of its nation-state borders is evident. The embodied nature of 'socially patterned' experience results in culture being perceived as the socially constituted reality which emerges through the interconnect of individual's authentic experiences (Baerveldt and Verheggen 1999). The interconnections created through expatriates and nationals working and socialising together, despite the limitations to non-Emirati autonomy at the expense of ensuring the minority population of Emiratis remain in control, will inevitably result in new and emergent cognitive states within society. Discourse is pivotal in bringing forth these new 
worlds of meaning, as it is through communication that the cognitive domains of individuals interconnect. Culture as an emergent cognitive process has been portrayed as the 'mental programming of the mind' (Hofstede 1980) or as the source of differential realities that contribute to individuals perceiving and judging socially distinguishing features as relative to self and a collective (Marsella 2005). Enactivist thought as underpinning the conceptual framework for this research, then shifts the foci from social constructivist views of reality as being socially pre-determined, to noting the embodied nature of experience involving communication and action where reality is created through enacted cognition. The challenge that arises in supporting cognitive enactment involving socio-cultural human plurality in project practice is to facilitate individual sensemaking for meaningful action, given co-existent differences in language and understandings.

The transnational flavour of economic practice and the increasing mobility of a diverse human workforce (Lee and Roth 2003) have been instrumental in the increase in socio-cultural mixes of discursive players in project environments globally. Such realities are creating unique human complexes involving a kaleidoscope of cultures and religion, re-defining what has traditionally be seen as 'complexity' in project practice (Cicmil, Williams et al. 2006). An emergent project team's culture, as integral to the fractal nature of organisational and national cultures, benefits from being perceived as being greater than the sum of its parts, with human nature existing as inseparable from culture and culture arising from the interconnect of individuals in communicative cognitive domains (Geertz 1973; Baerveldt and Verheggen 1999; Cooke-Davies and Arzymanow 2003; Whitty 2005). As Cope and Kalantzis (1997) have suggested, "As people are simultaneously the members of multiple life worlds, so their identities have multiple layers, each layer in complex relation to the others" (p.247). It is these realities that are the new project actuality which necessitate new and emergent responses for resilient solutions.

\section{Religion}

The significance of religion in this research context cannot be marginalised, particularly given the embedded nature of Islamic jurisprudence as reflected in Shari'a law and its guiding principles to national systems of governance in the UAE. In the literature, religion in relation to human differences has more commonly been identified as a dimension of culture (Hofstede 1980), however its impact on organizational or project practice has received limited attention (Kriger and Seng 2005). Whilst culture is usually associated with and confined to geographical locations, religion is more globally dispersed (Bonney 2004). Regional religious patterning however is evident, indicating for example, Islam dominating Middle-eastern countries and Judeo-Christianity being associated more with the western world (Ashkanasy, Trevor-Roberts et al. 2002; Kabasakal and Bodur 2002). Diversity in religious beliefs underpins a secular plurality that is evident in rituals and values of particular sociocultural groups with variations existing according to degrees of church-state separation. Islam as 
embedded in Middle Eastern systems of governance and formalized in Shari'a law, contrasts with western political systems which tend to reflect a more heterogeneous mix of religions with legislation being influenced by secular democratic ideals (Kriger and Seng 2005; Noland 2005). If we acknowledge cognition as a cultural process, then the inherent nature of religious beliefs when intertwined with models of national governance are ontologically inseparable. The interconnect of religion with other identified cultural dimensions of power distance, uncertainty avoidance, individualism-collectivism and masculinity-femininity (Hofstede 1980), should be seen to contribute to the social complexities existing in project environments comprising a human plurality that is socioculturally derived. As a source of influence on behaviour, religion cannot be overlooked as insignificant in the interpersonal tensions that exist in project practice. Complex human plurality should assume the presence of differences in belief systems; and when these beliefs are then legitimated in national systems of jurisprudence, the demand to adjust to different systems of accountability will be inevitable (Gelfand 2004) and need to be appreciated in the exploration of relevant project management responses.

\section{Socio-cultural difference}

Studies such as the GLOBE research program implemented by House, Javidan et al. (2002) which explored cultural dimensions of societal practices relating to perceived leadership qualities, reported patterns of social tendencies according to clusters of countries either sharing political history or being located geographically at close proximity. Whilst their results provide a snapshot of generalised regional social preferences, caution is needed in pre-empting or stereotyping behaviour, particularly given the greater permeability of nation-state borders today, the technology that enables greater awareness of global happenings and the multi-cultural mixes found in workforces world-wide. Nevertheless, results of the GLOBE study indicate that historically 'Arabization' and Islamic religion are intertwined and can be traced for at least a couple of thousand years. The countries in the GLOBE research cluster, Egypt, Morocco, Turkey, Kuwait and Qatar, all share a past history of being under the influence of Europe and the Ottoman Empire (Kabasakal and Bodur 2002) and today predominantly identify as Sunni Muslims although traditions between the countries do vary. Qatar, Kuwait and Egypt for example, have a higher percentage of Christians and Hindus in their populations, Kuwait and Qatar identify as Gulf countries and Egypt and Morocco are perceived more as Northern African countries rather than middle-eastern. According to Kabasakal and Bodur (2002) unifying socio-cultural determinants for this cluster include the Arabic language and ethnicity determined by ancestral descent, together with distinctive clothing, national dress, and dietary habits according to Islam.

The results of the GLOBE study for the Arabic cluster refer to nine cultural dimensions for societal practices, rating group and family collectivism and power-distance factors as high, while gender 
egalitarianism (the extent to which an organization or society minimizes gender differentiation) and future orientation (the extent to which individuals or organizations forward plan) are at the other extreme and are lower in ratings. Such portrayals however, fail to include a number of current day to day realities, particularly for countries such as the UAE. Historically the UAE in its present political form does not share the length of history of the other countries in the Arabic cluster, although they were under the influence of the British in the past. As mentioned earlier, indicators of country maturity levels reflect the 'newness' of the UAE and the uniqueness of infrastructure developments financed by oil revenue. As voiced by the problem owners in this research, although there remains a conservatism in the UAE population, 'students are pushing socio-cultural boundaries' and the organisational 'culture of bring in the money' drives a 'culture of immediacy - everything to be done straight away'. Despite the dramatic externally visible infrastructure changes in the UAE, the multicultural mix of workplace employees in the UAE and the exposure to different global ways of living will inevitably influence slower more subtle cultural change, much of which may be unconscious as individuals adapt to disturbances and obvious changes in the environment (Van de Ven 1986).

\section{The organisation}

The research in this dissertation was implemented in a higher education sector institute within the UAE. This social sector, alike other sectors in the UAE, particularly given the lack of educational institutions even 35 years ago, has experienced an enormity of change. To cater initially for increasing demands on education at all levels of schooling, the UAE 'imported' most education, both in form and policy which is not unusual for a country of lesser maturity. Yet today, while there is seemingly a clearer focus on what is needed to maintain the nation's development, national policy seemingly remains disjointed and at a 'novice' or 'initial awareness of process' stage, with a 'lack of planning and lack of experience as a developing country being indicative of immaturity of the organization and reflecting the immaturity of the country'. In the absence of a history of secular broad based education, the UAE has been guided by other more developed international education systems, including seeking of external accreditation and implementing foreign quality assurance systems. 'Home grown' policies as specifically relevant to the UAE population were perceived by problem owners, as being in their infancy, tending to be cautious in approach, inconsistent in nature and labile.

However, as Rust (2000) has noted quoting Aminata Soumare (1998) from an unpublished manuscript, this is quite typical of many such developing countries. Despite political independence for approximately 40 years, these 'younger countries' find that “...the national capacity for policy formation remains uninstitutionalised and episodic mainly because the policy foundation set by national procedures is quickly submerged under a flood of donor-generated country plans, sector studies, feasibility studies and staff appraisal reports that drive new investment and shape 
educational policy" (p.32). Perhaps where the UAE differs somewhat, is in the country's economic capacity to 'purchase' an array of the 'best' social sector practices and policies from nations with successful proven track records. The downside of this practice though is that multiple indiscriminate selections may be made, with there being a tendency to "look around the world and grab different things being offered' with a 'preference for things looking good, more of a façade', with contextual suitability or adaptability not always the priority, creating further challenges when multiple and conflicting project priorities co-exist.

\section{The organisational structure}

The Institute, in which the research described in this dissertation was implemented, was established in 1988 to provided education to a growing UAE national employable age population (19-65) which is expected to increase by $120 \%$ by 2025 according to information on the organisation's website. The organizational work breakdown structure (WBS) was based on a functional bureaucratic hierarchy (Mintzberg 1979; Turner 1999) and overall business practice was managed via an Arabic system of governance and operationalised by expatriates. Such a structure, combined with a suggested novice level of maturity, reinforced a risk averse socio-cultural working environment through promoting standardization of skills rather than encouraging creativity and innovation. A centralized administrative operations centre oversaw the running of Colleges in each of the seven emirates, maintaining a system of centralised control which limited the 'autonomy in decision making' at the College level, reducing creative and innovative responses to emergent issues.

This model of organization was typical of many public companies in the UAE, wherein the responsibility for day-to-day operational business practice resided with an expatriate community, whilst power of governance remained the remit of the UAE nationals. Over the past few years, however, the educational Institute in this research, became caught up in the global commodification and standardization of education, responding to pressures to incrementally shift to more divisionalised organizational structures, with corresponding changes in funding arrangements and emphases on forming strategic alliances with industry. The centralized operating core of the institute began to devolve the key part of its organizational functionality to individual colleges. This in turn impacted on the social architecture of the College with demands to shift from a more 'formalistic' to 'collegial' style of organizational architecture with an increase in 'business by projects' being evidenced. Responsibility and accountability were being devolved, yet power of authority as reflected in systems of centralised financial control and policy directives, remained the remit of the Central Services Administration. This structural-functional dichotomy within the organization created daily operational challenges that were compounded by miscommunication and multiple understandings in directives. Subsequent tensions arose from contrasting views, with these being further exacerbated by the presence of discursive dialogue resulting from the diversity in cultural 
backgrounds of employees whose different ways of knowing and understanding created ambiguities despite English being used as the language of communication.

\section{Quality systems and change}

The problematical situation as described in section 4.1.3, initiated the research exploration into factors perceived as contributing to an ill-defined messy situation. The implementation of a Quality Assurance (QA) system was not progressing according to project timelines, and despite the potential QA programs have in improving effectiveness and organisational resilience through reflective evaluative processes, there appeared a dichotomy where QA was being perceived as an externally driven administrative exercise focussing on accountability rather than QA being considered as potentially transformative. 'QA doesn't fit at all with organizational effectiveness'; 'It is considered more of an exercise to get through; not taken seriously, negative perceptions'. The challenges in defining 'quality' in higher education (as is likely in other industries) is the multiple stakeholder views as to what is being measured and the varied motivating factors involved. In the literature, QA has been noted as 'competing' with Quality Improvement (QI) programs, with the latter supposedly having the greater potential to facilitate effective change through an internal desire for improvement as opposed to externally mandated systems (Sachs 1994). Problem owners identified 'Too much external control'; 'Overwhelming, blindly collecting data, huge amount of data; slaves to forms'; 'Not well thought through, not meaningful; reports seen as imposition'; QA process driven to ameliorate external accreditation, indicating a dis-connect between system processes and adaptive behaviour.

The QA project as described in this research dissertation, alike other quality assurance or improvement systems, had the potential to facilitate change. However "...organisational success comes with unique and non-replicable cultural qualities: the human edge in service values, the quirky ingenuity of a design, the elegant simplicity of a process, the unique aesthetics or feel of a product, the hybrid creation of a product or a service for which there are no available benchmarks" (Cope and Kalantzis 1997 p.281). A culture which prefers certainty over uncertainty, living for the day rather than planning for the future and awaiting direction from an appointed leader who is expected to be charismatic but competent, contrast with contextual factors that are conducive to optimising benefit from quality improvement and best-practice performance programs. Continuous process improvement which is embedded within an organisation's functionality, where behaviour involves experienced intuitive and creative responses, assumes an upper level of maturity where process management is optimized (Benner 1984; APM 2008-9; SEI 2009). The research organisation's level of maturity, as reflecting the nation's was perceived as lacking established processes associated with embedded continuous improvement programs, demonstrating an overlaid QA system that set minimum standards but lacked clarity in process specificity. 
Organisational resilience is dependent upon a degree of freedom or autonomy to mutually respond to environmental perturbations and create change (Folke 2006), with innovative practice being key to adaptability of socially complex systems. QA as a superimposed system where control of process remains external, does not provide for the autonomy necessary for decision making that provides for adaptive responses to contextual demands. Organisational transformation is dependent upon cognition emerging through language and discourse from human interconnections. Evaluative processes in QA moving towards QI are potentially beneficial and may promote change, but if contextual constraints hinder the assimilation of knowledge alternative responses to facilitating adaptation will be necessary.

QA in the research organisation had become caught up in the process of decentralising a number of business initiatives to the Colleges. Although such strategy may be objectively interpreted as typifying attempts to improve College autonomy, project practice within the Colleges in reality was being perceived as devolving responsibility and accountability, but not the necessary freedom to selfregulate practice. Subsequent inter and intra-college tensions and competition contributed to a 'Lack of meshing between departments and college'; 'QA part of devolving responsibility to colleges but not necessarily fixing anything'; 'No system level agreements; colleges all differ in implementation, egos clash'. Ultimate decision making and control remained the remit of the centralised administrative system, limiting the potential change that QA processes can stimulate due to the constraints on implementing any new ideas or initiatives.

\section{Organisational power}

Problem owners identified a number of factors they perceived as hindering not only QA implementation and the effectiveness of the organisation, but over time, the long-term viability of the organisation. Socio-cultural and organisational cultural factors were noted as being inextricably linked with each other, with inherent power struggles and communication issues being pivotal to the situation. The realities of power struggles that relate to quality in education have been identified by a number of authors (Boreham and Morgan 2004; Burden-Leahy 2005; Small 2006), with Vroeijenstijn's (1990) early research being clear on the tensions that can exist: "The issue of quality in higher education is essentially political and becomes a site for struggle over competing ideological perspectives serving different personal and institutional agendas and interests" (p.23). The factors that made this situation somewhat different, was the multi-level power games that were contextually and culturally driven. On one hand, Colleges were being instructed by the centralised Arabic system of governance to assume responsibility for QA implementation, whilst on the other hand a constant flow of ad hoc directives impacted on progress, sending project priorities off on new trajectories. 'Lack of autonomy in decision making; management being undermined'; Many decisions being made centrally without planning - disruption at college level; strategies limited to control and 
destiny from central administration'. This contributed to feelings of powerlessness and perceptions that efforts to embrace QA as a means to improve College effectiveness was a pointless exercise. Within the organisation those with invested interests in maintaining some semblance of control, were perceived as doing so through creating a 'climate of fear' where 'behaviour is managed by control and fear'. This also added to the uncertainty in the working environment where risk taking was avoided as mistakes were not usually tolerated. 'There is a mind-set of, "don't take risks"'; 'Feelings of uncertainty'; 'Fearful of positions'; 'Can't be too vocal, no demonstrations, views can't be politicized no dissent'.

\section{Organisational leadership}

Specific cultural issues at the organisational level were identified by problem owners as being reflective of an Arabic working environment and as being different from expatriate's workplace experiences 'back home'. 'Process per se very difficult for nationals'; 'cultural attitude of the mind'; 'organization and UAE culture so different to majority of management team members' backgrounds'; 'College system culture driven by ego-centric, external display of wealth and importance'; 'system culture doesn't abide by regulation'; 'country has dictatorial style of management'; 'society not as stable; student culture and life is changing; identity crises'. Organisational leadership as a factor influencing the working culture then emerged as critical to the mode of operandi within project and departmental teams, the College and the Institute as a whole. As noted in section 4.1.2, the centralised Institute's management was predominantly Arabic, whilst leadership at the College Director level was usually western. The first Director in the College where the research described in this dissertation was implemented was Australian and the second Director, who held the leadership position in the last 6 months of this research, was American yet fluent in the Arabic language. Their leadership styles were quite different, and the distinguishing factors between the two will be presented and discussed further in section 5.1.3 on communication and 5.1.4 in relation to barriers and constraints to effectiveness. The interlinked nature of leadership, culture and communication filtered through the majority of the descriptions of the problem by the owners, relating effectiveness of the organisation directly to the manner in which College and system-wide operations were managed. 'Leaders set agenda - style and nationality combine to influence behaviour'; 'Leadership and effectiveness are directly related'; 'Need leaders of communication'.

Leadership in the Arabic cluster has been identified by Kabasakal and Bodur (2002) as being a 'paradoxical concept', balancing historical social traditions and expectations of a leader to be modest, consultative, charismatic and decisive, with the ability to make competent decisions and demonstrating worthiness in being 'selected' as the leader. The societies in the Arabic cluster therefore appear to have "...conflicting expectations from leaders... regarding the importance of ingroup relationships on the one hand and performance and improvement on the other hand" 
(Kabasakal and Bodur 2002 p.51). Paternalistic attitudes where the leader is expected to 'look after his flock' created tensions with expatriates of western origin from more individualistic societies, who found the more collectivist attitudes of the Arabic leaders were at odds with the more transparent shared governance models of leadership where success is attributed to effort and performance (Hofstede 2001; Kabasakal and Bodur 2002). Power as associated with leadership authority is therefore more accurately portrayed as a social property (Dowding 2008). This helps move the perception of the instrumentality of power as residing in the leader as agent, to viewing the structure-agent connect where power is socially embedded and influenced by cultural expectations of behaviour.

Leadership has been identified by the problem owners as needing to emerge or shift according to organisational needs and maturity. That is a 'one-style-fits all' approach is more likely to fail if the leader is not in tune with contextual and cultural dynamics. The College Directors were in a challenging position as they needed to be sensitive to the Arabic system of governance and the agendas of those in Central Administration, whilst assuming responsibility for the effectiveness at the College level which was staffed by a multicultural mix of employees. The leadership styles of the Directors would in part have reflected their socio-cultural backgrounds and experience within different systems of accountability. There was an imperative for both however to adapt to the working environment; to the perceived maturity of the organisation, to 'followers' diverse expectations of leadership behaviour and to the differing standards of accountability, the latter of which made more difficult to grasp due to shifting from work environments more individualistic to collectivist in nature (Gelfand 2004). 'Leadership style changes according to state of organization 'immature' - is in state of survival, needs more directive leadership,' 'intermediate' is reactive, but if 'mature', flexible leadership'. The College organisation was seen as 'stuck in an 'immature' stage due to the high turnover of staff - creating an immature management team, immature processes etc'. The working environment needed to be created for mutual benefit, but this was more difficult due to the wider contextual social cultural constraints. 'The Leader is at the helm, but the environment needs to be established so individuals are motivated'; 'Leader needs autonomy to lead'. A synergy was needed contextually to facilitate organisational self-regulation, but the 'supportive leadership' more conducive to innovative practice for incremental change and collective action (Van de Ven 1986), was constrained by the socio-political realities internal and external to the organisation.

\section{Organisational control}

The ongoing duality between desiring change to benchmark positively with western best practice education within the research organisation, was tempered constantly by the reluctance of Central Services administration to let go of control and share systems of governance. High uncertainty avoidance and the collectivist nature of the Arabic culture were limiting innovative practice, as 
delegation of responsibility did not share the necessary delegation of autonomy to allow risk and creativity. 'Individuals powerless to change; limited empowerment, need more autonomy'; 'Authority not aligned with responsibility'. In turn, leaders at the College level adapted their behaviour for selfsurvival and different standards of accountability to meet expectations of Central Services, applying directive approaches to organisational practice and reducing consultation to maintain some semblance of control over their own area of responsibility. This affected communication within the College, setting a scene for project practice in which project managers had limited decision-making authority without constant consultation with the College Directorship. This also contributed to an environment of uncertainty, low trust and caution where freedom to take risks was missing further contributing to creating a context that was not conducive to transformational and effective change. 'Leaders micromanage'; 'Lack of appropriate leadership, causes poor communication'; 'Reluctance [of employees] to assume leadership roles'; 'Need leadership for effective team work, to achieve goals, to help individuals work together'.

The 'power and politics' within the context are explored further in 5.1.4, but as Sense (2003) notes. '...long-term competitive advantage for project success is only achieved when the members of the project team effectively access the multiple learning opportunities and manage the political dimension' (p.107). To shift an organization from being 'unable to learn' to even acknowledging the advantage or benefits from learning, can be incredibly challenging (Lave and Wenger 1991). Whilst this necessitates visionary leadership with the 'expertise', 'creative thinking skills' and intrinsic 'motivation' (Walker 2004 p.65), i.e. the personal sources of leadership power, it is critical that to effect organizational culture change, this personal influence is seen combined with legitimate political and position authority and an ability to adapt to different standards of accountability. "...a company must work as hard to forget as it does to learn" (Hamel and Prahalad 1996 p.65); and this is difficult without legitimate leadership and a followership which recognizes the authority of the leader. Strategic planning or use of cognitive maps and templates, may facilitate the interpretation and integration of information for emergence and flow of knowledge through a multi-level learning organization (Crossan, Lane et al. 1999). However, to effect institutional change requiring shifts in culturally embedded practices, a combination of leadership skills and political authority is necessary to facilitate interrelationships critical to cognitive co-emergence. Problem owners perceived that leadership was pivotal to organisational functioning, with it being suggested that in this situation 'Effectiveness lacking due to leaders failing to set directions and purposeful action'. Leadership is embedded within a socio-political context with individuals inextricably linked with others and the environment and culture emerging in and through language. "The (institutional) context will define how the contrasting logics interactively organize people's, minds and actions" (Johannisson 2008a p.14). If authority of leadership - whether that be project management or organisational - is not 
autonomous in nature, then dis-connects between people and action are inevitable and new responses are called for.

The constraining factors in this project context included the multi-layered levels of authority, where action and inaction were influenced by socio-cultural factors associated with country and organisational maturity and disparities in expectations of expatriate employees in relation to communication patterns, leadership qualities and ways of doing business. Given these realities and real-world constructs of problem owners involved in this research, the issue of how best to strategically manage or respond to such contextual factors for improved project outcomes and organisational transformation has been considered and further explored in 5.2.2. The increasingly divergent nature and boundary permeability of individual life-worlds being seen in globally complex working communities are producing a multiplicity in meanings from human and environmental interconnections (Cope and Kalantzis 1997). The socially derived project complexities in this research dissertation reflect an increasing multicultural human plurality and unpredictability that project management strategy will need to address for successful outcomes.

\subsubsection{Language, communication and cognition}

For projects as social process to be effective conduits of organizational change, strategic management of human interaction and the development of practitioners who learn through reflection are necessary for co-ordinated action. The concept of 'intersubjective mutuality' (Sikka 2008) which reflects Jürgen Habermas's (1981) theory of communicative action, assumes a consensus of action based on an overarching metanarrative that brings all together through agreed dialogue. Predictably, his pragmatic theory presupposes a commonality in language and discourse, thus understanding, as the foundation upon which organizational business and project management practices could be seen to be based. Habermas's model of 'communicative action' would seem to assume a socio-cultural homogeneity where mutuality in human interactions is culturally determined. However, findings from the research in this dissertation suggest that a 'consensus action' model based on facilitated interpretation in project management is more relevant for culturally complex organizations comprising socially disparate project teams. There is a 'need for leaders for effective teamwork; to achieve goals; to help individuals work together'. In the process of striving for effective and sustainable change in and through projects, the tensions that exist in social contexts comprising multiple discursive realities and involving complex power relations necessitate acknowledgement and emergent management. Language differences and varied cultural nuances create a context where consensus in understanding cannot be assumed, yet through accommodating for difference, making sense despite such complexity may be possible. 
Consensus in meaning and 'shared' understandings are elusive realities in socially complex entities, but through recognizing this fact as the first step in exploring the dynamics of the environment, project coherence may be facilitated. As suggested by Baerveldt and Verheggen (1999) “...cognitive mechanisms should not be searched for 'within the head' of a person, but rather within the discursive or conversational interactions between persons" (p.185). Too frequently the assumed 'information sharing' until an 'acceptable level of understanding' or 'mutual understanding' (Loosemore and Al Muslmani 1999) is reached, is alluded to in the literature. But this concept of mutuality, together with the assumption that non-verbal semantics of communication will be transmitted in a manner that is 'mutually understood', reflect a simplistic interpretation of communication and demonstrate a lack of cultural understanding particularly between the extremes of western and Arabic ways of thinking and doing. Hand gestures, physical appearance, eye-to-eye contact, gender differences in what is acceptable communication vary in meanings between cultures. It is quite acceptable in the western world for example, for a woman to offer her hand first to a man to shake in introductions or as a parting gesture, but in the Arabic business world, the man takes the lead and the more conservative members of society would find it offensive if a woman made physical contact with a male non-relative. There is a need for 'strategy to accommodate different ways of communicating, different assumptions etc'; 'Women as second to men is general attitude outside the home'. There are 'religious pressures eg covering' which affect communication between genders making non-verbal communication involving reading of facial expressions difficult. 'Males are more free'. There are 'variations in [acceptable] behaviour from emirate to emirate'.

Despite the possibility that a westerner being able to speak Arabic may improve communication in middle-eastern project teams comprising Arabs and other nationalities, the suggestion that it is 'important to speak Arabic to work effectively in The Gulf' and that failure of English speaking expatriates to learn how to communicate in Arabic is a sign of 'linguistic complacency which creates the potential for misunderstanding and conflict' (Loosemore and Al MusImani 1999), reflects some misguided conclusions about reasons behind expatriates not learning or grasping the Arabic language. Many workplaces operate in English in the UAE, and in the organisation in which the research for this dissertation was implemented speaking of Arabic was actually discouraged. Teachers were disciplined if found speaking in Arabic to students, as such behaviour was seen as counterproductive to assisting the national students to learn how to communicate in English effectively. English is the predominant language of business for day to day communication due to the multiple cultural groups that reside in the UAE and the import of western business and education. Arabic language as the UAE national language, despite the different dialects has been identified as contributing to a unity in association with Arabs from other nations (Kabasakal and 
Bodur 2002), but it is the Islamic religion which has been identified as the glue that binds (Khaldun 2005).

Ibn Khaldun (2005) as an early $14^{\text {th }}$ century Islamic Sufi philosopher, used the Arabic word ijmâ for this 'glue', which is defined as consensus - or the cohesive element that informs judicial process and safeguards Islam. The group feeling of the Arabic society which is noted for its collectivist nature, i.e the 'assabiyah' is seen as the cohesive force of the group or group spirit, a conscience that has "...its own specificity and collective aspirations ... the tension that animates it and impels it ineluctably to seek power through conquest' (Talbi in Khaldun 2005 p.xv). Assibayah is "... a concept of relation by sameness, opposed to the state (dawlah) based on relations of difference or complementarity, and to religion (din), which alone supersedes it" (Anderson in Khaldun 2005 p.xv). Learning to speak the Arabic language may facilitate communication in industries where English is not the primary language of business and the project team comprises predominantly workers from Arabic speaking countries; but a sense of belonging in the middle-east is culturally derived and associated with a tribal history which spans centuries. Arab rhetoricians divide language into insha (norm and tradition) and khabar (event); where Insha is not questionable nor qualified, i.e. it is usually a command or query, whilst khabar can be discussed, confirmed, qualified or refuted by others (Khaldun 2005). Expatriates in the UAE do not 'share' the tribal history of the Arabic people. They are seen as workers that have knowledge and skills which can be imparted for the benefit of the developing nation. 'Expats expected to give to system and country and move on'. Language of insha is unique, and recognising the disparities existing in a culturally diverse project teams will be a more effective means of accommodating for difference than attempting to speak the Arabic language to 'belong'.

Multiple realities co-exist, but "...the reality we have in common and in which we find ourselves, is neither a world that exists independently from us, nor a socially shared way of representing such a pregiven world, but a world itself brought forth by our ways of communicating and our joint action" (Baerveldt and Verheggen 1999 p.185). That is, both understanding and cognition are emergent through language, but 'sharing' an understanding when language and culture are discursive is a concept that is not an accurate representation of real-world constructs.

Whilst the essence of what is perceived as 'Arabic culture' cannot be negated, given the increasingly dominant use of English as the language of business and higher education in the UAE, it is interesting to consider the influence this factor has on 'observer's' realities within the socio-cultural context. Enactivism sees that it is "...only within a linguistic domain that an observer can emerge. Language enables human beings to relate to their consensually constituted reality as if it were a reality independent of their own conduct" (Baerveldt and Verheggen 1999 p. 200). Meaning arises out of 
interconnections in consensual domains, with the project entity as coupled with organisation and environment, potentially providing a co-operative contextual setting to facilitate emergent cognition.

Consensus may involve agreement to work together thus contributing to project progress. In relation to QA systems, such consensus has the potential to facilitate emergent cognition to transform the organisation from a state of being to a becoming ontology, but only if the values of quality are grasped and internalised by all stakeholders. The challenge in managing project domains comprising culturally plural mixes of actors is to achieve some semblance of accommodated understanding to avoid the miscommunications that see people heading off down different paths of action. As suggested by Whitaker (1995), “...the word 'shared' is misleading because it passes by the operational and experiential closure of the interacting systems. A consensual domain can therefore better be conceived of as a "co-operative domain of interactions" (p. 2). A pragmatist view would suggest that consensus may be reached through rational debate, with this Habermasian concept assuming a mutuality in language. Maturana (2002) however, more accurately portrays consensual domains as being determined by co-ordinations of behaviour where individuals are structurally coupled with one another and individual meaning arising through observer descriptions taking place in language. In such a context of 'becoming' "...there are no (other) realities to bridge to, only new worlds to literally enact through negotiations with good-willed others" (Johannisson and Olaison 2007 p.75). A 'respect for radical Otherness' (Derrida 1992) may contribute to agreement on action or a way forward, but assuming a consensual domain equates with agreement on meaning is incorrect. Consultation with all stakeholders is necessary to identify, acknowledge and accommodate for differences to "...enable 'action to improve'..." (Checkland and Winter 2006 p.1436), with SSM as selected for this research, providing a methodology that is potentially transformational. The project reality is that praxis is socially complex comprising multiple real-world constructs, and in the research problematical situation it was perceived that 'staff need to be consulted; more opportunity to contribute to decisions in relation to change'; 'more dialogue [is needed]'. The leader should be 'engaged with others', developing an array of responses that emerge as sufficiently flexible to accommodate the non-linear yet patterned behaviour of interconnected stakeholders.

The assumed structure-agency divide perpetuated by behaviourist and constructivist theories of cognition has traditionally driven theories of learning, separating the object from experience. However with suggestions that activity, collective activity and situated experience are seen as pivotal to cognitive development (Leont'ev 1981; Vygotsky 1986; Lave and Wenger 1991), a shift in thinking to note the distributed nature of cognition and language as the actual social reality has emerged (Maturana 1978). Wenger (1999) acknowledges Lave's (1988) contribution to socio-cultural cognitive theory, when stating that "...social practice is the key to grasping the actual complexity of 
human thought as it takes place in real life..." (p.281). Engeström's (2001) theory of expansive learning then extends situated learning theory to describe learning as taking place "...in a changing mosaic of interconnected activity systems which are energized by their own inner contradictions" ( $p$. 140). Wenger (1999) also presents a social theory of learning that acknowledges human consciousness or self-awareness as being "...fundamentally constituted by processes of both identification and negotiability [where] our communities and our economies of meaning are inherent aspects of the social fabric in which we define who we are" (p.213). Learning in these "communities of practice' thus assume an interconnect between contextual components.

It is enactivist thinking however, through aligning 'autopoiesis and cognition' that the learning theory foci shifts from knowledge acquisition being constructed from subjective experience of being situated in a world, to having the learner being embedded in context where cognition is embodied (Varela 1987). In this portrayal, social interaction provides the necessary connect between cognitive systems, with language and communication bringing forth a world of culturally patterned existence (Gergen 1985; Varela, Thompson et al. 1991; Baerveldt and Verheggen 1999; Maturana 2002).

Given this thinking, suggestions that "...the key to overcoming the problem of inter-cultural communication is to develop sensitivity to cultural diversity..." to "...encourage[s] people to erode the behavioural differences which interfere with the communication process" (Loosemore and Al MusImani 1999 p.95) is a somewhat idealistic concept. Cultural diversity certainly needs to be acknowledged, but attempting to 'remove' culturally patterned differences is not only unrealistic, but even if possible, would negate the value found in human diversity. Culture as language is emergent and the structural coupling of individuals in a project team embedded within an organisational and socio-cultural context provides fertile soil for embodied cognition. What is needed to make sense in socially complex project environments are 'strategies to involve and accommodate all stakeholders'; 'non-static systems [of communication]'; 'more dialogue'; 'strategy to accommodate different ways of communicating, different assumptions' and more specifically in relation to knowledge management systems in workplaces with short-term employment contracts, there is a need for 'policy.. to counteract disconnections created when people move on; 'transience needs better management policies'.

\subsubsection{Enacting cognition: Barriers, constraints and leadership}

As suggested in 5.1.2 and 5.1.3, consciousness enacted from interrelationships of mutual reciprocity becomes somewhat messy given the socio-cultural mixes of human plurality increasingly being evidenced in project environments. The complexes of real-world constructs in contexts of human 
plurality will see a "... struggle for the future ... not... between social and economic classes but the structure of consciousness" (Bateman 2006 p.2). Resilience and adaptive capacity of the project organisation moves beyond responding to an environment to one which is able to sense perturbations, shape the context and manage change through enacting cognition for transformational change (Folke 2006). Aligning the self-perpetuating characteristics of project organisations as complex adaptive systems with Hall's (2005) seven criteria for organisational autopoiesis, a mature project organisation may be seen as autopoietic in nature if exhibiting characteristics of being:

- Discriminable from its environment;

- Comprising components as determined by the organisation;

- Complex;

- Dynamically self-regulating;

- Capable of intrinsically producing its own components;

- Able to maintain sufficient and necessary self-produced components;

- Self-sustaining over time.

However, in addition to these autopoietic self-regulatory mechanisms the structural openness of an organisation is necessary for sensing and creating contextual change for ongoing resilience. As suggested earlier in this dissertation in section 2.4.2, the project entity potentially provides the means for organisational adaptability through acting as a conduit to provide the structural openness for organisations embedded in context. Enacted cognition for emergent knowledge is key to the continual and evolving processes that see learning, change and adaptation in and through project practice as pivotal to sustainability. It would be naive to assume however, that emergent change is unhindered. Contextual constraints and barriers, complex power games and tribal politics can all erode positive social processes that promote organisational learning through project praxis. Whilst strategic intervention may limit the damage being done by counterproductive political dynamics, the challenge is to promote a functionality into project praxis where adaptive renewal becomes the constant.

\subsubsection{Knowledge as enacted cognition}

The complex nature of the project organization as a social entity with multi-level interconnected learning platforms, present numerous challenges for the project manager who is expected to grapple within a dynamic environment where learning and politics co-exist. Dialogue facilitates the 
enactment of cognition and the differences found in human plurality provide the foundation for change. If autonomous project praxis is privileged at the expense of relinquishing bureaucratic control, it is more likely that the voice of all stakeholders become "...a source of authority for the one who embodies it, and thus power is centred in each being, rather than in a socially created power structure" (Hazen 2001 p.22). The culture of the project organisation may be seen to emerge through language and dialogue, where relationships based on a mutual reciprocity facilitate cognitive enactment that potentially liberates and transforms rather than oppresses (Freire 1970). In discussions on knowledge management in an era where 'knowledge as a definable resource' and asset of the firm dominate, the issues arising from relationships between knowledge and power as “...social processes that create and constrain each other in intricate ways" (Ekbia and Kling 2003 p.2), cannot be overlooked.

Problem owners in this research have suggested that 'discontinuity and gaps in knowledge' were created by 'destabilized management structures and ongoing CS infrastructure changes'. A bureaucratic organisational structure sustained centralised control and despite CS moving to devolve some responsibilities to the College level, ultimate power remained embedded in the structure-agent connect with the person and political power residing with the Emirati leaders as reflective of the socio-political context. The Institute exhibited a 'bureaucratic organizational and operational structure' where the 'Chancellor is in control'. Effectiveness of the organisation was being restricted with individuals feeling 'powerless to change, limited empowerment, need for more autonomy' with there being identified a need for greater 'share governance, shared responsibility'. Knowledge rather than being seen as enacted cognition was being 'used' 'as power' through the 'withholding of information' and controlling of 'knowledge as expertise' through selectively limiting individuals consulted for advice. 'Power to' effect change was being misappropriated through the perpetuation of a model of 'power to' sustain a social-political status quo.

\subsubsection{Contextual constraints and barriers}

Barriers to organizational learning have been identified in relation to the transfer of knowledge (Zack 1999; Dixon 2000; Szulanski 2003; Walker 2004) and in relation to the organizational learning culture and individual behaviour (Cavaleri and Fearon 1996; Argyris 1999). Individual, organizational, contextual, behavioural, cultural, and process factors all have the potential to hinder the creation of emerging knowledge assets. However, many of the knowledge management (KM) models are instrumental in nature portraying knowledge as an objective entity which can be codified and measured, with transfer and utilisation subject to management controls. Szulanski and Jensen (2004) identify the need to ably manage both knowledge and situational characteristics in the quest 
to overcome 'stickiness' in knowledge transfer. 'Causal ambiguity' where there is uncertainty or inability to relate and use outcomes for new approaches is then related to the 'absorptive capacity' of an organization where it is suggested that effective use of knowledge is proportional to the organization's capability “...to recognize the value of new external information, assimilate it, and use if for commercial ends" (Jewell and Walker 2005 p.114). 'Unproven knowledge', where knowledge is used once but effectiveness in a different situation is either not recognized or is doubted, together with causal ambiguity are thus related to the characteristics of the knowledge itself. Furthermore, absorptive capacity as a situational characteristic supposedly affecting knowledge transfer has also been associated with other influential factors including motivation (from both the source in efforts to share and from the recipient in the willingness to accept), trustworthiness (of the knowledge source) and the retentive capacity (of the recipient to 'institutionalize the utilization of new knowledge') (Szulanski and Jensen 2004).

Whilst such portrayals of knowledge as a definable asset demonstrate a recognition of the value found in the human element of the project organisation, such views can be limiting in as much as they perpetuate the instrumentality of knowledge as being an entity able to be 'withheld', 'used' or 'destroyed', in the quest for ultimate power and authority. These representations restrict the view of knowledge to more linear, objective models, rather than seeing cognition as emergent through language and as consequential to the social process of project praxis. Barriers in this sense may be more accurately portrayed as those created by people whose thinking is fragmented and disconnected, where minds do not meet, hindering the emergence of cognition and culture which arises through discourse. The challenge for the PM is to create an environment which dissolves such barriers, one where "...there arises one mind, where they are all one unit, but each person retains his or her own individual awareness" (Bohm 1992 p.87). Given the culturally diverse human element certainly does not make this process simple, with problem owners identifying 'difference' as the factor which is difficult to manage. There were perceived differences in the research context in 'time, styles of planning, ways of communicating, assumptions, priorities and perceptions of what is important' creating a social disparity that was seen as limiting rather than beneficial.

As argued by De Long and Fahey (2000), “...organizational culture is widely held to be the major barrier to creating and leveraging knowledge assets" (p.113). An organisational culture of risk avoidance and centralised control was perceived by the problem owners as a limiting factor in enabling change through restricting action and stunting innovative practice. The feelings of 'uncertainty, fear for positions, lack of autonomy and a climate of fear', when combined with a constancy in change resulted in staff, 'let all wash over; why bother?; powerlessness, give up trying to be heard', with a feeling that they are 'being forced to work with change - no choice', with the result being an organisation that is seemingly 'hanging on by the fingernails'. An inertia to change or 'burn- 
out' is particularly evident when 'change for the sake of change' which is 'ongoing, unplanned, constant' with 'nothing allowed to run and be analysed', contributing to de-motivation and avoidance of instigating or being involved in new initiatives. This not only hinders the enactment of cognition for learning and change but restricts the 'unlearning' of ineffective practice that maintains status quo and reduces the adaptive capability of the organisation.

Swieringa and Wierdsma (1992 in Cavaleri and Fearon 1996) have identified four strategies as being directly related to an organizations inability to unlearn:

- Collective blindness - acting as if the problem does not exist;

- Collective avoidance - shaping of views and opinions to conform to only those that the organization wishes to acknowledge;

- Collective reluctance - insecure individuals and teams producing only what they think the management wants;

- Collective ignorance - lack of technical or political knowledge despite recognition of a problem.

All four factors, but particularly the first three, were evident when reflecting upon problem owner's perceptions of the situation as described in this dissertation. Not only is the 'absorptive capacity' of an organization negatively affected by such constraints but the renewal capacity involving reorganization and development for resilience and adaptation (Folke 2006). Collective blindness, avoidance and reluctance to act reflect high uncertainty avoidance and risk averse social group characteristics as identified by Kabasakal and Bodur (2002) in their research on the Arabic cluster of countries. Such organisational behaviour limits the emergent cognition necessary for proactive responses to the unexpected through hindering recursive 'learning-unlearning' cycles and constraining action that may shape the environmental context. Continual ad hoc change can leave 'no time to be proactive' so that 'operations are retroactive' with the organisation seemingly in a state of damage control. The imperative for organisations striving for resilient states is to '...learn to manage by change rather than simply to react to it...' (Folke 2006 p.255), with collaborative teamwork and co-ordinated work efforts in project practice providing a structured approach to achieve such states.

Competition rather than collaboration does not facilitate the re-generation or re-organization that is required for whole entity adaptation and resilience. The competitive nature of the UAE society as suggested by the problem owners, was mirrored in the project organisation where for example, reward systems for 'best' teacher, 'best' performance, 'best IT solution' were measured on ability to impress, rather than on substance or quality of educational programs. Such 'competitiveness 
obstructs interests of all stakeholders' and was combined with a preference for 'external displays of wealth and importance'. There was a 'lack of effective performance management systems to deal with poor performance' and 'no mechanism for rewarding exemplary performance' rather 'competitiveness between colleges' was encouraged. This socio-cultural reality did not foster relationships based on mutual trust which are critical to adaptive co-existence, thus limiting the capacity for renewal which is possible through tolerating and managing change (Folke 2006). As has been suggested by Sveiby (2001), “...reward systems that encourage individual competition will effectively block efforts to enhance knowledge sharing" (p.348), the latter of which is integral to the learning necessary for shaping and adapting to changing environments.

The societies in the Arabic cluster identified by Kabasakal and Bodur (2002) as exhibiting high uncertainty avoidance and collectivism, tend to draw upon interdependent relationships with family, kinship or other social group collectivities as a self-protective mechanism to cope with the unknown. Trust is associated with family and 'in-group' relationships that have been developed overtime, and comprise individuals who demonstrate preferential behaviour for those who are seen as 'belonging' to the group. Expatriate employees in a middle-eastern country such as the UAE have a transient presence, and when they not only lack the same language but predominantly the same religion as the Arabic nationals, it is not unexpected that the concept of 'trust' as in the western or Anglo world, is missing from social and working relationships. "High groupism and hierarchy of relationships that is dominant in these societies indicate that trust is only shared with a small group of people" (Kabasakal and Bodur 2002 p.52). When trust is missing from the workplace, feelings of disempowerment, increased vulnerability and exploitation as have been identified by the problem owners involved in this research dissertation, are difficult to shift. Work actions and communication patterns become 'fear driven', limiting emergent cognition and stifling learning processes through disconnecting individual relationships. 'Trust' in a project organisation directly relates to knowledge flow and successful outcomes in project practice (Munns 1995; De Long and Fahey 2000), with working environments based on inequities and control-command models of management negating attempts at establishing and fostering trusting work relationships (Kadefors 2004). When distrust dominates or outweighs the presence of trust in project practice and systems of control are privileged at the expense of autonomy, then an environment of fear, scepticism, cynicism, wariness, watchfulness and vigilance will limit the establishment of positive interdependent relationships (Lewicki, McAllister et al. 1998). For the emergence of 'trust' in working environments that operate on fear, recognition of the complexities involved and the interpersonal dynamics existing, can be seen as the first step forward in promoting the collaborative practice needed to foster the emergent cognition necessary in successful project practice. 


\subsubsection{Complex power games}

Knowledge as emergent and dissipated, indeterminate and evolving (Tsoukas 1996; Orlikowski 2002) is enacted through cognition and is better enabled when relational power dynamics reflecting sectional interests focus on autonomy as opposed to control. When tribalism and asymmetric power struggles surface in complex projects, particularly those involving strong technical elements and diversity in social interrelationships, project failure is frequently the outcome (Livingston 1990; Cicmil and Hodgson 2006). Ignoring the realities of social inequalities existing in complex project environments as has been identified in the socio-cultural context of this research, together with the impact cultural disparities have on communication patterns and emerging cognition, will limit the adaptive ability of institutional systems ultimately threatening long-term organisational resilience. The particular challenge in project management practice arises when the nature of power is socioculturally embedded and mechanisms of control exist to reduce risk and protect vested interests.

The existence of high power distance factors together with the Islamic influence in defining gender roles, serve to perpetuate systems of male-dominated patriarchal societies within the Arab world (Kabasakal and Bodur 2002). Foucalt (1977) suggests that relational power as vested in human existence needs to move beyond a model of dominion where the instrumentality of power as being held and wielded dictates individual identity if organisational transformation is to be effected. Power as a producer of knowledge as 'power to' is seen as having the potential to facilitate cognition as a social process through accommodating for diversity and difference in social groupings of a disparate nature, where definitions of power vary as greatly as do cultural backgrounds. Much of Foucault's (1982) work focuses on the relationship between power and truth and the circular nature of power which is perceived not as a commodified entity, but rather as being exercised through individuals as vehicles of power. The challenge is to circumvent the 'tribal affiliations' that exist within a sociocultural context, particularly in countries whose social history and traditions are tribal in nature.

Socio-political processes exist in most organisations and inevitably involve "...historical legacies that are built into local custom and practice... [creating] ...an important friction on transformation" (Clark and Geppert 2006 p.342). Arabic society is recorded as having the earliest civilisations in the world (Kabasakal and Bodur 2002), and the UAE society as a traditionally tribal society has a dynastic history extending centuries prior to its more recent political form of 37 years. Family name is considered important and influential in all social and business circles, with inequalities existing from tribal rights to positions, property and privilege. Hilder (2004) has identified tribes as having:

- Authority figures who exemplify the values of the group;

- Followers who accept those values as 'facts'; 
- The group is glued together by attention exchange between the members;

- Tribal members are 'people' - outsiders are considered to be subhuman (p.635).

Whilst some aspects of these characteristics may seem extreme, the realities of civilisation today are "... always and everywhere marked by the fundamental difference between urban and primitive, producing a tension that is also an interplay between nomad and merchant, desert and city, orality and literacy" (Lawrence 2005 p. x). The dynamics between social groupings, whether tribes, family, cultural, create a social space that is not only predictive of behaviour and predictive of expected behaviour, but the mutual reciprocity between structure and agency reflect a social differentiation which is contextually specific. Organizational rules will influence agency strategies, but the same rules structure the powers of the different agencies (Dowding 2008). The project organisation needs to move beyond models of obedience and conformity which limit proactive and adaptive responses as "...all inequities are ultimately dysfunctional in organisational and social terms" (Cope and Kalantzis 1997 p.282). However, 'tribalism' with all its self-survival instincts, is commonly contextually embedded within societies and social organisations, creating the demand for adaptive models of governance if organisational capabilities are to be transformational.

\subsubsection{The politics of tribalism}

In the introductory section of the translated version of Al Muqaddimah (Khaldun 2005), Dawood has noted the interdependent relationship of the dynasty and state (dawlah) which exists in traditional tribal societies as is the UAE. The close ties of such relationships will see the consequential collapse of the government state if the dynasty is destroyed. The group feeling or solidarity which is consolidated as group consciousness or assabiyah is most commonly associated with clanship or tribes, although long family friendships (usually spanning decades) may include non-family members politically as 'in-group' relations. "A group with a powerful asabiyah achieves predominance over other groups and by the same token the leading family or ruling dynasty within one or more groups will be that whose immediate blood relationships inspire or command the strongest asabiyah" (Dawood in Khaldun 2005 p.x1ii).

The heroic nature attributed to leaders of these tribal groups can exist even after their deaths, particularly given the reality that leaders in Arabic nations are "...the center of all important ideas and strategies; policies and programs emanate from him" (Kabasakal and Bodur 2002 p.51). This has been evident in the UAE with the status of Sheikh Zayed bin Sultan Al Nahayan as the first President and Ruler of the UAE who died in November 2004 remaining as a presence in glorified memory. The current social and political achievements of UAE are clearly attributable to Sheikh Zayed and his time 
as leader of the country since 1971 and reference to this fact in the public domain is still a common occurrence several years after his death. Ultimate political power of the ruling family resides with the Al Nahayan family with Sheikh Zayed's son, Sheikh Khalifa bin Zayed Al Nahyan having been appointed as his successor soon after Sheikh Zayed's death. The Chancellor of the organisation in which this research was implemented was a member of the ruling family. The personal, position and political power (Yukl 2002) ascribed to him, together with the bureaucratic nature of the organisational structure, ensured that ultimate power and authority remained his remit.

As presented earlier in this discussion, the UAE population in numbers are a minority compared with the high proportions of expatriates working and living throughout the country. Yet political power and authority clearly remain with the Emirati population, with citizenship only being available to national Emiratis, and senior government ruling positions only being available through appointments. Control is maintained by limiting the degree of autonomy granted to individuals and organisations, with methods of control being embedded in structural relations within society. No national system of voting which is inclusive of the expatriate population exists and ultimate decision making resides with members of the royal or ruling family. The national population themselves however, do not operate on a level playing field. Social inequities do exist, with these being primarily associated with wealth of the emirate in which different members of the population live, the genealogical distance families are from the royal family and the historical tribal affiliations of social groups. As noted in the research by Kabasakal and Bodur (2002) on leadership in the Arab world, "...proximity to the leader is important and the close circle of the leader finds the ability to act as advisors to the leader" (p.51). The tribal nature of business practice within the research organisation was raised by problem owners throughout the research process, with clear associations being made with affect on organisational functionality and project process. Inequalities in relationships where power is embodied in social constructs and environs are a social reality. The issues this research has attempted to address involve the exploration of the dynamics of socio-cultural difference, where projects as social process will benefit from acknowledging and exploring the epistemological pluralism that underpins the structure of our world (Healy 2003). Tribal behaviour is not restricted to the days of the huntergatherer, rather are alive and well in today's political and social environments globally. Tribalism may be evidenced wherever there are authority figures who share similar values with a number of followers, where followers support without question the values of the leader, where there is some form of 'glue' to keep members together - whether religion, language or social exchange, and where there are 'others' who are not associated with the leader and the group, so are on the outer (Hilder 2004).

Ibn Khaldun's (2005) Arabic philosophical writings as recorded in the Muqaddimah have been noted as being "...the earliest attempt made by any historian to discover a pattern in the changes that occur 
in man's political and social organization" (Dawood in Khaldun 2005 p.xxxix). Political authority and the associated power that is embedded in ruling tribal groups is connected with the ability of the leader to provide for the needs of his followers, in parallel with systems of justice based on Islamic principles (Chapra 2006). Rulers are seen as God appointed or determined, so that questioning their decisions is considered sacrilege. The restrictions to access of knowledge in Islamic societies which contribute to social inequalities and thus power of influence, is not derived from the Quran which sanctions rights to knowledge. Rather it tends to be found in the Hadith where access is usually based on gender, that is, what is deemed 'suitable' for women (Al Hashimi 1996). It was Sheikh Zayed who decreed that all Emiratis, male and female, should have free access to education and as leader of the country was respected for his decision. Today, the only restrictions on educational access are family derived, with more traditional families still tending to select the daughters who are allowed to attend higher education and/or seek employment, and those who are to remain home to look after family or marry very soon after schooling has finished.

The Chancellor of the Institute in which this research was implemented was a UAE national and member of the ruling family. The Vice Chancellor was an appointed UAE national of Iranian descent. Ultimate decision making remained the remit of the Chancellor and Vice Chancellor, with appointments 'below' this level being perceived as 'selected to fulfil or maintain socio-cultural status quo; people who are different are pushed out'. Regulatory systems in relation to appointments, administrative processes and academic issues existed within the College, but it was not unusual for decisions to be made by those in authority outside these guidelines, with a generalised feeling that the 'system culture doesn't abide by regulation'. The 'country has a dictatorial style of management' and 'ways of acting are culturally derived'. The endurance of UAE as a nation draws upon the Emirati's 'power to' control the expatriates who visit to live and work in the country through both structural and political agency means. Some expatriates alike UAE nationals, may use their positional, personal or political 'power to' affect change, but this is more likely to occur if action and outcomes are perceived as mutually beneficial. 'Interest prevails' according to 'historical locations' with those financially wealthy (as are a high proportion of the UAE nationals), more likely to maintain control in non-democratic societies (Dowding 2008). Inequalities in power distribution are the norm in the Arabic society, with hierarchical structures as existing in families where the father is the leader and 'head', being mirrored in social institutions and businesses (Kabasakal and Bodur 2002). This is a reality that necessitates a proactive adaptive response rather than resistance in complex project management.

The feelings of being 'threatened, worried about job security and the unpredictability in life' were reinforced in the research organisation by sudden unexplained contract terminations, changes in policies and 'things made taboo, e.g. sites blocked', with such factors being perceived as adding to 
insecurities and feelings of disempowerment. The organisation being 'stuck in "immature" stage', contributed to tribal behaviour exhibited by not only those in leadership positions, but by those employees who formed associations with other 'less powerful' players to provide the sense of belonging and 'assabiyah' that can provide strength in adversity. As suggested by Hilder (2004) “... the tribal response to attempts to solve problems is a major factor in the failure of complex projects with a strong technical element" (p.642). He notes that affiliations and associations need to be identified to reduce the constraints that destructive or counterproductive behaviour can have on project progress. That is, the human element cannot be ignored.

Working with socially disparate groups where alliances are multi-layered and fluid and self-survival is the priority, a complexity is created which is difficult to sift through and manage to provide the transparency necessary for effective communication to enact cognitive processes. Conflict and tensions can be productive in social groups that incorporate democratic ideals into discourse (Clegg 2006), but when "...debate is stifled in the name of order...power overwhelms imagination" (p.856). Inequalities arising from indiscriminate resource allocations which are enabled or disabled by social structure, can limit the capacity people as agents have to respond and affect change; and as creativity is stifled, innovation is avoided and ego-centric behaviour dominates. In the research College context, an expatriate 'assabiyah' based on a victim or powerless mentality existed which was seen as needing to be replaced with one where there is 'greater co-operation, shared vision, all in-together; a need for engagement between leader and others'. Difficulties in achieving these were related to power being predicated to those privileged with the autonomy to act within a structure that was socio-culturally and politically determined and protected. As identified by Bill and Leiden (1979 in Kabasakal and Bodur 2002), "The relationship between family connections and power distance is even more evident in the shaykhdoms of the Arabian peninsula, where the core of the political elite always consists of members of the ruling family" (p.47). Working with such reality in the UAE is the project actuality, where superficial functional change may emerge, but deep-rooted sociocultural change is less likely.

As Foucault is quoted in Sikka (2008), 'Humanity does not gradually progress from combat to combat until it arrives at universal reciprocity, where the rule of law finally replaces warfare; humanity installs each of its violence in a system of rules and thus proceeds from domination to domination'. The UAE is ruled and dominated by the national Emirati population which strives to maintain nationstate control and autonomy despite the increasing permeability of the country's borders and disproportionally large expatriate population. Tribal behaviour works on protecting kinship, and assimilating 'outsiders' into socio-cultural groupings based on historical clans is not an option, as their system of national tribal rule 'proceeds from domination to domination'. Organisations within the UAE also reflect the maturity of the country, with the 'power of monetary capital' (Hilder 2004) 
remaining the remit of the Emiratis due to democratic political authority as is known in the western world being absent in a culture based on inheritance and Arabic tribal traditions. The 'power to' affect change as expatriate workers with limited autonomy may therefore seem elusive. But it is proposed that 'power to' empower Emiratis who are structurally embedded in the UAE socio-cultural context to demonstrate adaptive behaviour through emergent change, may be facilitated by human interconnections guided by evolving leadership.

\subsubsection{Leadership as the way forward}

A change in College directorship during the research described in this dissertation provided an insight into the problem owner's perceptions as to the affect - positive and negative - organisational leadership can have on system performance. 'Leadership at college changes and causes culture change of organization'. Problem owners compared the styles of the two leaders, identifying communication strategies and leadership qualities as relevant to the effectiveness of the organisation. There was a perceived 'need for a leader with vision, trustworthy, open-door, openness to change, strong, able to treat others with respect'; 'leaders of communication' and it was felt that the 'academic skill set of the directors as leaders do not always equate with effective management'.

The first Director whom had given permission for the research in this dissertation had years of previous experience in a similar position. He was perceived as being consultative, but as 'managing individuals to reduce the power of the group and limit debate, more controlling and acting from fear'. This more 'directive' style of leadership could be attributed to the maturity of the organisation, i.e. as being 'in a state of survival [thus] needing more directive leadership', or could be a reflection of the perpetuation of tribal behaviour where reduced autonomy to lead resulted in more controlling behaviour to protect self.

The second Director who was younger and less experienced was perceived as 'indecisive, naive, as allowing others more powerful to decide [and exhibiting] shared governance only when wanting to dissipate responsibility'. He was seen as more superficial and portraying an image of 'gloss', and as 'being manipulated [and] controlled externally to the college' by those in authority in Central Services.

As suggested by Bourne and Walker (2005) leaders as project managers need to “...develop robust relationships with project stakeholders to ensure successful delivery of the project outcomes and ...this requirement calls for a set of skills, beyond managing and leading, that enables the PM to work within the culture and political environment of the organisation..." (p.1). Problem owners suggested that the Emirati leadership outside the College entity, that is the stakeholders from Central Services 
(referred to as the 'client' in this research), 'set the agenda - [with the] style and nationality combined influencing behaviour; being non-consultative, a poor communicator; managing by fear, control and power'. And the change in leadership at the College level was seen to break 'processes of communication', with problem owners feeling that with the new Director there was a 'lack of support for themselves as managers; feelings of being undermined and fearful'. It is agreed that a PM needs skills 'beyond managing and leading' to 'establish an environment so individuals are motivated'; 'more collegiality needed, need to feel valued'. Whilst 'robust relationships' may possibly emerge amongst expatriate problem owners involved in project practice, the realities of the tribal nature of the society, the inherent Arabic cultural characteristics and the maturity of the country combined with other cultural constraints as raised earlier to create a number of barriers that were difficult to surmount.

The socio-political nature of the project organizational entity lacked the autonomy necessary to freely enact the human cognition existing as a pluralism of ideals, indeterminate and fluid. The role of the project manager should be perceived as not attempting to 'control' human involvement rather should be seen to facilitate an environment where management emerges to work with and through the existing social complexity. Through acknowledging the non-static and interconnected human element in project practice, where incremental autonomous action underpins discontinuous action, adaptive governance is seemingly a better fit to manage the complex power games existing in context.

The problem owners' perceptions of leadership qualities necessary for increasing organisational effectiveness, included a leader who is 'visionary, a good communicator, able to articulate vision, adaptable to maturity of organisation, engaged with others, [as having] increased autonomy, more consultative with everyone for decision-making, transparent, management by "walking"'. The Arabic members of society as noted in Kabasakal and Bodur's (2002) research, paradoxically expect a leader "...not to stick out and have modest styles on the one hand, and on the other hand be a man with a 'miracle', who leads his followers to their ideals" (p.51). This dichotomy is a constant, adding to the tensions created in a project organisation when staff from diverse cultural backgrounds work together. They are assumed to bring their own cultural expectations of preferred leadership behaviour and styles. Yet despite a diversity in difference created by the socio-cultural expatriate mix existing in the research organisation, a number of cross-cultural similarities have been recorded as a result of the Global Leadership and Organizational Behavior Effectiveness (GLOBE) research project (House, Javidan et al. 2002). After research across 61 societies world-wide, five dominant leadership characteristics were identified as preferred: charismatic, autonomous, humane, self-protective, participative and team-oriented (Kabasakal and Bodur 2002). Team-oriented and charismatic were the two outstanding characteristics identified in the Arabic cluster of countries. Comparing these 
with the problem owner's perceptions reveals a degree of consistency with both global and Arabic preferences.

Table 11 Comparing Problem Owner leadership preferences with project GLOBE results

\begin{tabular}{|l|l|}
\hline $\begin{array}{l}\text { Problem Owners' perceived preferences for } \\
\text { leadership characteristics }\end{array}$ & $\begin{array}{l}\text { GLOBE cross-cultural perceived preferences for } \\
\text { leadership characteristics }\end{array}$ \\
\hline Visionary & *\#Charismatic \\
\hline Good communicator & Humane \\
\hline Autonomous & Autonomous \\
\hline Consultative & *\#Team-oriented \\
\hline Transparent & Self-protective \\
\hline Management by 'walking' & \#Participative \\
\hline
\end{tabular}

* Arabic perceived effective leadership styles

\# Anglo perceived effective leadership styles

The two main differences between these comparisons is the preferred visionary capacity and leadership transparency which are desired by the problem owners in this research inquiry. Given that the problem owners were members of the College management team of which a high proportion originated from ' ${ }^{6}$ Anglo' countries who tend to 'emphasize performance and look towards the future' preferring a 'lesser reliance on formal rules and procedures' and 'more equal power distribution' (Ashkanasy, Trevor-Roberts et al. 2002), this is not unexpected. However, although the Arabic preference for a leader who is charismatic rather than visionary is consistent with the low future orientation scores and cultural tendency to idolise the leader (Kabasakal and Bodur 2002), it has also been identified in the Anglo cluster of countries as a perceived effective leadership style. Zagorsek, Jaklic et al (2004) have suggested that the GLOBE research project has demonstrated that "...charismatic leadership may as well be a universal phenomenon" (p.27). A team-oriented leadership approach also 'fits' with the collectivist nature of the Arabic society; yet although 'teamoriented' was not stated explicitly as a leadership characteristic by problem owners in this research, the importance of 'shared governance', 'leadership for effective team work', 'need for team building'

\footnotetext{
${ }^{6}$ 'Anglo' as defined as a research cluster in the GLOBE research study, included Australia, Canada, England, Ireland, New Zealand, South Africa (white sample) and the USA. Ashkanasy, N., E. Trevor-Roberts, et al. (2002). "The Anglo Cluster: legacy of the British empire." Journal of World Business 37: 28-39.
} 
were all identified as factors seen as important for organisational effectiveness. These results reveal a degree of consistency in thought despite the more 'individualistic' as opposed to 'collectivist' nature of the Anglo clustered countries, reinforcing the call for caution when making comparisons and drawing conclusions based on homogenous cultural patterning.

Leadership capacity to manage project practice in socio-political contexts where power to effect change is constrained by a multiplicity of factors, requires different conceptual approaches in viewing the actuality of projects. A shift from instrumental models of project management to appreciating the critical nature of social processes involved, has the potential to unravel some of the complexities arising from historical cultural structure-agency interconnections evolving country and organisational maturity and disparities in thinking and doing which exist in project trajectories. Maintaining flexibility in project practice encourages spontaneity in action in response to unpredictable change. Projects as complex human activity systems emphasise the interconnected nature of the human element as the project actuality, with cognition arising through language from discursive sociocultural mixes of human capital. Allowing for emergent social processes rather than attempting to prescribe set formulas to business practice, removes the increasingly irrelevant scientific 'cook-book' approach to unpredictable social problems and supports the notion of expansive learning for organisational transformation (von Hayek 1974; Engeström 2001). Change and adaptation as inherent to the nature of the project organisation as a complex adaptive system, requires emergent leadership to maintain a balance between change and stability to ensure incremental transformative processes can facilitate the ontological shift from a state of being to one of becoming. This emergent leadership is not prescribed, nor transferrable as a definable entity, rather is a style of leadership which evolves through structurally coupled mutually reciprocal relationships as contextually determined. This is the leadership relevant for new and emergent project realities.

\subsection{Projects as social process}

Emergence as a property of projects as 'complex adaptive systems', reflects the cognitive nature of the entity, with embodied experience serving to produce meaning and learning being the product which assures organisational resilience. Such a becoming ontology moves beyond the perceived adaptive nature of self-organising project systems where survival involving emergence to sustain a state of 'being' dominates. Projects as social processes involving a complexity of human interrelations fluctuate between stability and instability with uncertainty and unpredictability being exacerbated by the dissipated nature of cognition which emerges from disparate patterns of human connections. 
Project management research and practice will benefit from paradigmatic shifts in thinking to appreciate the critical nature of projects as socially complex, where difference as contextually embedded requires appreciation and strategic management for improved and emergent project outcomes.

\subsubsection{The complexity of difference}

Perceiving projects as complex human entities is supported by postmodern thinking which places difference and indeterminacy as dominant forces in the construction of our world (Cooper and Burrell 1988). Meaning emerges through complexes of human interactions where cognition is enacted through discourse and the structure-agency connect involves an inseparable mutual reciprocity. The capability of project organisations comprising culturally diverse mixes of actors to configure, assimilate and foster emergent knowledge for organisational resilience, is dependent upon strategic approaches to optimise cognitive enactment. The inherent tensions that exist in project practice when assumptions that underpin communicative dialogue are discursive will be better managed if project actuality as self-referential, knowledge as emergent and the benefits of patterned difference are acknowledged.

Problem owners in the research described in this dissertation noted the differences in understanding and communication styles of employees within the organisation and project teams, creating a social complexity which was noted as being difficult to manage. Differences in 'perceptions of time; styles of planning; ways of communicating; assumptions; priorities and perceptions of what is important were factors identified as impinging on project practice. Given the interconnected nature of the human element in the project as social process and the discursive socio-cultural nature of stakeholders involved, facilitating a working environment that provided for optimal learning and change was challenging. Language disparities, despite English being used as the main form of communication, reflected multilayered levels of subjective interpretations which were at odds with one another and were being strongly influenced by the Arabic system of governance. The project organisational 'structure' as embedded within a national cultural context, was seen as inextricably linked with the 'agents' whom were also contextually embedded, with the resultant social complexity being inherent in day to day functionality. This complexity did not originate from multi-firm partnerships, international alliances, cross-cultural partnerships or joint ventures as has been identified in other project research (Loosemore and Al MusImani 1999; Gupta and Govindarajan 2000; Clark and Geppert 2006; Bresnen 2007; Ambos and Schlegelmilch 2008; Grisham and Walker 2008); rather project complexity arose from and within the project entity as interconnected and embedded in context, providing an insight into the social nature and ecology of project practice. It is 
thus the context of project practice and the complexity created by social-cultural differences in this research which emerged as being critical to a better understanding of project actuality.

The research in this dissertation has supported the call for scholars to "...pay closer attention to the relationships between projects and their environments" (Söderlund 2004b p.664) facilitating a shift in emphasis away from structured 'one-size-fits-all' project management models, to focussing on strategic approaches in working with projects as socially complex processes. The unpredictable nature of the project trajectory has created the demand for exploration into the complexity arising from the social interactions that exist in project practice (Winter, Smith et al. 2006) to enable different conceptualisations to the more rational and finite models of project management.

The recognition of the holonic nature of projects and the critical nature of managing part-whole relationships are instrumental to the challenges being faced in project practice at the turn of the century. This is particularly so given that projects as social process are more than the sum of their parts and are embedded in an organisational and in-turn socio-cultural context which comprises many elements. The interconnect and dynamic relationships existing in and between all components and the environment create a volatility in an era of uncertainty, where the realities of the individualism being promoted in today's world of 'late modernity' (Höijer, Lidskog et al. 2006) are compounded by characteristic innovative, creative and risk taking behaviours. Such norms as being more typical of societies individualistic in nature are in contrast to the Arabic research context as described in this dissertation, where social collectivity rather than individual difference was preferred. As has been suggested by Bhagat, Kedia et al (2002), the "...individualism-collectivism dimension of cultural variation is the major distinguishing characteristic in the way that the various societies of the world analyse social behaviour and process information" (p. 208). Differences in mother-tongue and associated underpinning behavioural assumptions, together with contrasting views on life priorities and preferences, remained as dominant influences throughout project communication, despite the dominant use of English as the language of preference in this research context.

Problem owners not only identified socially derived differences as factors impacting on organisational effectiveness, but suggested the constant 'ongoing, unplanned change' where staff were 'forced to work with change - no choice', and the level of 'uncertainty from lack of response to queries', as being influential in creating an environment dominated by individual disempowerment and inertia to change. Feelings of 'change burn-out', 'let all wash over', 'why bother?' revealed a dichotomy whereby the massive infrastructure changes being evidenced in the country and being desired by the 'whole' project organisation, were being stifled under systems of governance which served to maintain control and operational status quo rather than permitting risk-taking behaviour of individual 'parts' which would enable innovative and creative practice for developing maturity. 
'Dissent was not permitted', yet 'competition was encouraged' and 'external image, having the 'best' of everything' was important. Privileging the autonomy in project practice that is needed to facilitate a greater transparency in communication for learning and change amongst socially diverse project players, was missing within the project organisation. The complexity arising from social differences and the ambiguities in decision making were compounded by systems of governance which perpetuated bureaucratic methods of control. Managing such a multiplicity of part-whole relationships within the project context called for an emergent form of leadership, one which not only evolved in adaptive response to changing social dynamics, but one whose strategy recognised and accommodated for the fluid nature of all interconnected components.

The discontinuous nature of change in the research project context thus not only called for a leadership approach which could promote a degree of continuity in practice despite the social disparities, but one which could operate contextually drawing upon differences to create a 'project collectivity' of purposeful identity. Van de Ven (1986) has suggested that there is a limit to the degree of complexity that people can cope with, yet it is proposed that if cultural differences can be acknowledged and negotiated, more positive and resilient outcomes are likely in what Cope and Kalantzis (1997) have referred to as today's “...era of civic pluralism and total globalisation..." (p.281).

Project leaders, alike national and global leaders, are facing social contexts which are "...highly complex, constantly changing and difficult to interpret" (Zagorsek, Jaklic et al. 2004 p.16). Cognitive realities are culturally specific and emergent as the social make-up of the environment changes. This calls for flexible, non-concrete strategic approaches to guiding the project as social process towards learning for effective change and adaptive resilience. The multiplicity of factors which impinge on project praxis in reality, are frequently outside the bounds of individual control. Yet the first step in moving forward whilst acknowledging an ontology of becoming, is to recognise the value inherent in existent human plurality. The ambiguities which will arise from human interconnections within disparate social contexts are the project actuality. It is the development of an organisation's capability to assimilate and accommodate for these differences which can be effected through projects, as their adaptive nature provides the structural openness necessary for promoting the contextual interconnectedness which is integral to learning, making sense and bringing forth new worlds of meaning. 


\subsubsection{Project management strategy}

Strategy in relation to project management theory and practice has been associated with three main concepts of project practice: projects as subordinate to the parent organization, projects as autonomous agents coupled with a parent organisation and projects as organisations in the absence of clearly defined governance (Artto, Kujala et al. 2008). Strategy in this context assumes the project entity is definable and reified, which is in contrast to what has been presented in the research described in this dissertation, wherein it is suggested that the project is a social process which is emergent in context through interconnections of actors with each other and the environment. Strategy in this sense necessitates new conceptual approaches to project management - particularly given the emergent states of the unknown and the social complexes and ambiguities being faced in a more globally mobile world. Project management strategy becomes indistinguishable from knowledge strategy where the reality of project praxis, no matter the 'goals and expected outcomes', is to enable the emergent cognition which underpins the knowledge creation and learning necessary for organisational change and transformation.

The human socio-cultural kaleidoscopes which may exist in projects as process have a potential wealth of 'new' knowledge being created as a result of diverse mixes of interconnected world constructs. It is 'in and through' projects that real socio-cultural change can be effected. Development and maturity of the organisation and ultimately the nation may therefore be seen as dependent upon realisation of the critical nature of the human-environment interconnect in project praxis. Strategic approaches to accommodate for difference to counter-balance the powerful interplays that surface when the status quo is challenged or questioned are pivotal in shifting an organisation from a state of adaptive self-regulation, to one which is constantly becoming, emerging and shaping the environment for adaptive resilience.

Problem owners in the research described in this dissertation identified 'retroactive operations' and 'disconnections created when people move on' as necessitating 'policy... to counteract knowledge loss' with a 'lack of KM systems to capture knowledge' and a 'lack of policies, procedures and guidelines' negatively impacting on the effectiveness of the organisation. Capability to create and respond to environmental perturbations was perceived as being hindered by an absence of organisational policy to strategically manage the communicative disconnects resulting from disparate ways of knowing and the transient nature of the expatriate employ. The constancy of change and the realities of knowledge-system gaps resulting from failure to recognise the value residing in the human element of the organisation, was compounded by 'retroactive operations', 'competing priorities' and the organisation seemingly 'always in a state of response and reaction', with no time 
'to plan or be proactive'. Socio-politically, those in leadership positions were expected to be seen to consult, but radical ideas or dissent from those with limited authority were not favourably received. New ideas were better accepted if perceived as originating from those in management, but the 'lack of policy and long-term directions and implementation' and 'the lack of strategic planning' resulted in ad hoc and inconsistent approaches to operational practices. Functional strategies were seen as 'limited to control and destiny from central administration' rather than the systems of governance providing a 'sense of direction...with purposeful action'. Strategy was seen as needing '...to accommodate different ways of communicating, different assumptions etc', with it being subsequently suggested in this discussion that the implementation of effective project strategy is dependent upon more empathetic approaches to everyday business practice to effectively manage the complex dynamics which underpin project praxis.

The 'new' project actuality notes the embedded nature of the project as social process. We are in an era of globalization where, as raised by Law and Urry (2004) the "...complex, elusive, ephemeral and unpredictable" (p.404) not only dominate our social world on a global scale, but the impact may be felt in the day to day realities of individual world constructs. New strategic approaches and "...tools for understanding and practicing the complex and the elusive" (p.404) are thus required. Fixed instrumental approaches to project practice as we enter the $21^{\text {st }}$ century are alike social science methodologies which may have been useful in explaining social realities of the past, but today such finite, linear approaches are inadequate when coping with the uncertainties and non-linear project paths which typify project complexity. Today's realities involve enactments which are 'fleeting', 'distributed', 'multiple', 'non-causal', 'chaotic' and 'sensory' (Law and Urry 2004 p.403), with projects as social process more accurately reflecting the embedded nature of project praxis in unpredictable, fluid and changing contexts.

The research described in this dissertation pre-supposes that similar complex socio-cultural mixes exist within project and organizational cultures in other nation states. Enabling an emergent interactive model of project management which facilitates communicative action through dialogue to accommodate diversity and difference for assimilated meaning, will benefit not only the organization, but the individual and ultimately the nation in quests for sustainable business practice. Organisational survival and resilience may therefore be directly related to the adaptive capacity of the complex whole to accommodate for and manage diverse cognitive processes that not only reside within project teams and organisational structures, but in the interconnections of the larger sociocultural environmental context. Project management is now in an era of plurality, where reality is differential, cognition is indeterminate and sensemaking ambiguous (Clegg and Ross-Smith 2003; Cicmil 2006; Höijer, Lidskog et al. 2006). Management of projects as 'process' as the new actuality subsequently necessitates different approaches to optimise the knowledge creation which is integral 
to an organisation's capacity to adapt. Engeström (2007a) has made reference to “...developing shared purpose across organizational units and divisions, and coordinating work among various skills and competencies along the value chain" (p.4) as defining process management, but this methodology tends to assume a homogeneity in the human element of the organisation, and may not be as successful if aimed for in project organisations comprising discursive human plurality. Problem owners in this research noted that 'having a team comprise different personalities is same as anywhere in the world', but 'adding the different personalities to the different cultures ... adds layer of complexity' which the project manager as leader 'needs to acknowledge upfront'. 'If there is dysfunctionality in a group - combining different cultures and personalities' will only make it more complex and challenging.

Enabling the cognition which arises through language for meaningful interpretation of project purpose may therefore be better achieved through facilitating a project culture which acknowledges the value of diversity in difference. Problem owners saw the responsibility for this, lying with the project leader; 'leader needs to create the environment; leader needs to pick the right blend of people'. Discourse which draws upon both verbal and non-verbal communication may promote consensus to map the next direction to follow in project implementation. But assuming unspoken interpretations of the project trajectory are shared is a misnomer. Understandings are subjective, building upon a vast cultural history of experience, knowledge and assumptions which influence the interpretative process. Project managers as leaders need to work with stakeholders to promote discourse that result in some semblance of agreement in ways forward whilst recognising that meaningful interpretations are more likely to be assimilated rather than shared. The 'major problems with communication' in the research project context were identified as 'not necessarily the nationalities' alone. Rather problem owners perceived identifying differences in personalities as crucial, due to the complexities arising from the additional 'overlay of cultural differences' in the project context and the power games that created an environment where communication worked 'very much on fear and blame i.e power/authority/blame'.

Project-based business practice as a solution to organisational complexity and pressures being exerted from the external environment have been reported in research by Martinsuo, Hensman et al (2006) and Hobbs, Aubry et al (2008). In addition, the research described in this dissertation has raised the issue of further challenges being faced by project managers when the complexity is inherent to the project entity itself. That is complexity not arising from technical specifications, rather from human and social processes that comprise the project actuality. Whilst the technical side can bring its uncertainties, success and failures, it is the unpredictable nature of the human element, which is made more complex when a culturally disparate human mix of stakeholders comprise the project team, that real challenges surface. As such, attempting to define strategy to 
accommodate these differences and the inherent power games that are acted out in context, will only in part offer potential solutions to the problems at hand.

Project strategy needs to be flexible. It needs to work with the given socio-political culture to promote emergent change from within. Cooke-Davies and Arzymanow (2003) align successful project outcomes with a project culture which is conducive to effective implementation, yet we need to be cautious in suggesting that the skills of a project leader should include the ability to bring about broad-based cultural change. Acknowledging the interconnected nature of organisational and nation-state socio-political cultures, it is possible that incremental cultural change may be facilitated at the project level, but it is more likely that 'emergent leadership' which is adaptable to the organisational culture whilst being sensitive to the value found in a diverse mix of project stakeholders, will be the most successful model to pursue. Van de Ven (1986) suggests that in relation to complex environments "The strategic problem for institutional leaders is one of creating an infrastructure that is conducive to innovation and organizational learning" (p.605). However, it is even more challenging for the project manager who is not in a position to 'create an organisational infrastructure' when the socio-political context is pre-determined and the PM is required to strategically manage the project actuality for effective solutions. The tensions that exist within organisational structures, where power games involve the limitation of autonomy as a means to maintain control, will inevitably influence change processes and the project paths which are taken. As new players enter and exit the project arena, power relations realign, new games are played and subsequent new tensions emerge (Hobbs, Aubry et al. 2008). Such realities call for strategic approaches to accommodate for the constancy in change, where discontinuity in cognitive processes for learning and adaptation are tempered with continuity of embedded cultural realities that determine the ways of organisational operation.

Potential solutions for effective project practice reside in a project manager who is "profoundly' conscious of the multiple human dimensions being enacted within a socially and politically complex project environment. "...no matter how hostile or challenging the environment in which the organisation exists, its consciousness and prosperity are foremost about people and not about objects, so that the organisation's aim must be measured in terms of its ability to intentionally satisfy primary human needs, which are not only needs of humanity, but also needs of being" (Edgeman and Fraley 2008 p.706). Project managers thus require not only 'status' and 'authority' to accommodate for alternative opinions and understandings through enabling discourse and the voicing of multiple

7 'profound' - 'having and applying intellectual insight that extends far beyond the boundaries of common knowledge' drawn from W. Edwards Deming's System of Profound Consciousness and defined by Edgeman, R. L. and L. A. Fraley (2008). "A system of Profound Consciousness: Building beyond Deming." Total Quality Management 19(7-8): 683-707. 
interpretations (Boddy and Paton 2004), but the skills and intelligence to work sensitively and intuitively to circumnavigate the power plays which surface throughout a project trajectory. The differential factors of religion and culture intertwine in a project context, resulting in a complex epistemological pluralism. This in turn determines individual identity through the realities of power and politics being woven through a social landscape which struggles to balance autonomy with control. Problems which have been created by a particular level of awareness or consciousness can only be resolved or improved upon through seeking and enabling new and different levels of emerging cognition. Facilitating human interconnections for the emergence of cognition through language and discourse, will assist in the synthesis of new and evolving states of consciousness from the connect of multiple real-world constructs. Edgeman and Fraley (2008) suggest that "..once a threshold has been crossed creating a critical and strategic mass of profoundly conscious individuals within the organisation, a sort of diffusion occurs so that profoundly conscious teams form and operate, and a profoundly conscious organisation begins to emerge" (p.686). Diffusion is then possible inter-organisationally, with project praxis having the potential to facilitate these interconnections through providing the structural openness for an organisation to be aware of environmental perturbations, as well as facilitating knowledge creation through project stakeholder interdependencies with others internal and external to the project organisation.

Problem owners emphasised project strategy needing to address the issues of 'employees needing to be and needing to feel respected and well resourced... if not, people won't put head up in case they 'get shot'; people won't take risks' and instead, for self-survival 'become self-focussed; don't move forward', thus limiting the adaptive ability of the project organisation. Again the issue of leadership at the project team or departmental team level was identified as being instrumental in facilitating the necessary supportive environment which could promote a greater transparency in communication and 'permission to make mistakes and act'. Action by project team leaders, despite the overwhelming socio-political environmental constraints, could seemingly be seen as having the potential to initiate development of 'profoundly conscious teams' through 'managers creating their own 'department culture' for survival - gives a sense of belonging'. Over time, these teams could in turn contribute to the emergence of a more 'profoundly conscious organisation', given more stable conditions where the human element is of a less transient nature than is found in expatriate communities.

The majority of leadership theories are individualistic as opposed to collectivist in nature and emphasise the role of the follower as having particularly responsibilities or expected behaviours (House and Aditya 1997). Such models have then been universalised and presented as transferrable from context to context, despite remarkable differences in socio-cultural and political contexts. Models and associated instrumental tools of project management such as the Gantt-chart, CPM, 
PERT and PRINCE 2 have also been promoted as providing technical solutions to sift through some of the business complexities being faced today. Whilst successful outcomes have been evidenced particularly in projects with clear goals, linear paths and in singular company settings, today we are evidencing multiple interconnections of businesses and projects on an international scale (Söderlund 2004b). In an era of complexity and ambiguity, stereotypical answers and predictable models may provide a degree of certainty which helps one cope with the unknown, similar to a protective 'defence mechanism' (Van de Ven 1986) which may contribute to survival. Yet successful outcomes demand a greater flexibility and appreciation of the influence of the human element in project practice and the impact diversity has on the project trajectory.

Project strategy, alike project models or organisational policy, needs to move beyond set formulas to work with the reality of the social nature of projects as embedded in context, where management and policy is recognised as non-transferrable from one national context to another (Phillips and Ochs 2004; Söderlund 2004a). Sveiby (2001) suggests that the competence of people underpins formulation of strategy, but in these discussions knowledge as associated with competence development is portrayed as a definable resource which can be converted and transferred nine different ways within an organisation. Again, although such representations contribute to visualising the value found in knowledge creation, separating knowledge as an entity perpetuates the structureagent divide that in actuality does not exist if we acknowledge the embodied nature of cognition.

Project outcomes would seem to benefit from project strategy that focuses more on process, human interaction and enabling of action, as opposed to lengthy time spent on vision building, structured planning and attempting to gain 'shared understandings'. Such 'process strategy' would provide a more flexible approach to project implementation to accommodate for the ambiguities and complexity inherent to today's business world. Enactment of project management focussing on the interplay between human action and institutional contexts, will promote the formulation of project strategy which is socio-politically aware and more sensitive to local customs. Projects as social process thus profit from strategy which facilitates the blending of world constructs in project implementation, through promoting co-emergence of new realities that move beyond threatening one's cultural extinction at the expense of another's survival. Change therefore emerges from within, from the interconnects of individuals embedded in context, where understanding arises through discourse and 'rational communicative action' “...dismantle[s] the ideological systems that traditions embody and that, as a consequence, hold freedom in bondage " (Habermas 1981 p.14). Human action is enabled in and through project actuality, where action is seen as pro-active and empowering, facilitating change through being "...role-breaking or custom-defying..." challenging the status-quo protected by rules and directives (Wolin 1988 p.200). New and emergent collectives are thus formed through projects as social process to move project actuality beyond project 'practice' 
which "...signifies doing things competently according to the appropriate canons" (Wolin 1988 p.200) to a process of action which enables the emergence of new realities as we grapple to find understanding in complexity.

Shifting the focus of project strategy to process and action supports the notion of projects as human activity systems, when if privileged to function autonomously, may facilitate enacted cognition and sensemaking for emergent consensually validated worlds. The challenge for project managers particularly working with projects that are inherently culturally complex, is to facilitate a connect between action and knowing whilst working with disparities in realities and cognitive ontologies of project stakeholders. Project strategy that emphasises synergistic management of part-whole relationships will more ably accommodate for dissimilarities in domains of reality arising from variances in individual's praxis of living which exist in the absence of overarching metanarratives. Furthermore, project strategy which recognises projects as social process will provide the enabling mechanism for enacted cognition in the flow of pragmatic knowledge. A project organisation's capability is then 'measurable' by evidence of the emergence of new knowledge and ways of doing which underpin the incremental change necessary for resilience and sustainable development despite complexity in group dynamics.

Lack of meaningful project strategy fails to provide justification for ongoing seemingly unplanned change. The discontinuous nature of project-based change (Engeström, Kerosuo et al. 2007b) demands clarity in communicating purpose. Problem owners in this research dissertation, identified lack of 'reasons given for change; everything done at a whim' as being associated with a lack of strategic action, which was seen as leading to 'uncertainty, feeling out of the loop, can't be bothered - inertia' to change. Change was seen as 'OK, but need to know reason; this needs to be communicated'. The Arabic socio-political environment of the research project context was dominated by systems of bureaucratic control. Communication with those of Arabic descent was considered different to the more transparent forms of communication that expatriates of Anglo origin were accustomed. Problem owners suggested that 'when dealing with Arabs - listening is dependent on how much they actually respect you; they will listen more if you have a name with ${ }^{8}$ wasta, depends on who they are, perceptions of others.' Perceived qualities of a leader also differed. Team building, empowering others to share responsibility, attempting to draw upon strengths of others, were not behaviours expected of those in leadership positions according to Arabic traditional norms. 'Leaders need to be seen to be decisive - if you admit you don't know, then it is seen as weak'; 'trying to be inclusive and empower people without making ultimate decision, is seen as weak'. The paternalistic style of leadership as identified in Kabasakal and Bodur's (2002)

\footnotetext{
${ }^{8}$ Arabic word meaning 'influence' - more commonly associated with tribal influence and influence through personal connections particularly with those closest to Arabic leaders
} 
leadership research in the Arabic cluster of countries, was supported by problem owners perceptions, with hidden undercurrents in communication seen as limiting the transparency needed to fully enable the interconnected cognitive processes. 'There is a need to be more dictatorial in the Arab world; Saving face underlies most communication'. An environment where distrust from 'fear', 'action based on false information' and 'communication as not honest' functioned to maintain a 'false front' dominating and acting as a barrier to the human interconnect which underpins knowledge creation (De Long and Fahey 2000).

Such realities necessitate creative and strategic approaches to project praxis to facilitate the discourse pivotal to organisational resilience. Engeström (2007a) has suggested “...negotiated knotworking as an emerging way of organizing work in settings that strive toward co-configuration" (p.5), with such strategy offering positive means to enable collaborative action through learning for change. This becomes more challenging however, in socio-cultural contexts which are dominated by more rigid systems of rules and centralised bureaucratic control which serve to limit the autonomy necessary to create a project environment that can sustain collaborative 'knotworking' or flexible interconnections between actors.

The obvious differences between western-Anglo and Middle-eastern ways of life when interconnected within the one environmental context, permeate much deeper than the more obvious superficial levels of differences in diet, dress and mannerisms (Marsella 2005). The 'western' ways of doing which sat comfortably with problem owners involved in the research described in this dissertation were not only at odds with Arabic ways of thinking and doing, but could be seen as a "...threat to a traditional fabric of life rooted within a culturally constructed reality embedded in a religion that penetrates all aspects of daily life and behaviour" (Marsella 2005 p.657). Systems of control to protect the familiar dominated the state of play, creating tensions that limited the negotiative processes that underpinned sense-making activities necessary for pro-active project responses.

Project strategy for dealing with complexity as inherent to the project as social process, is itself complex, non-linear and ill-defined. It requires a flexibility in approach that privileges action and process over practice. Strategy which views projects and project management as social phenomena, constructed in and through interactions amongst a multiplicity of players, will benefit from acknowledging that cognition as language is enacted through the interconnects of individuals embedded in an environmental context. Discourse facilitates cognitive enactment, yet individual understanding or making sense of the world round us, “...presupposes a set of positive cultural and individual prejudices we can never get beyond" (Gadamer 1981 in Sikka 2008 p 237). The challenge for the project manager is to rethink project strategy as being replicable across contexts, to focus on the emerging nature of project praxis, where process is fluid and outcomes are dependent upon 
fostering the interconnect of discursive social players. Conceptualising the emergent nature of the project process and the enactment of cognition through discourse, will optimise the diversity of difference, whilst providing a flexibility which will more ably circumnavigate the political nature of socially complex project environments.

\subsection{Limitations}

The research in this dissertation has achieved its first aim in exploring the significance of a specific socio-cultural context in project practice for effective change, and in part met the second aim in suggesting adaptive strategic PM responses are called for to accommodate for cultural disparities in human capital. However, specific techniques per se have not been identified as initially proposed, as it was concluded that such tools perpetuate more positive, rational approaches to project practice. This would negate the main thesis in the research that has focussed on alternative conceptualisations of project practice to accommodate for the emergent nature of cognition found in non-linear, dynamic project praxis. The research also aimed to focus on the $3^{\text {rd }}$ quadrant of the DPM Practice to theory framework as described in section 1.2, with another shift towards the $4^{\text {th }}$ quadrant. Again, although this research has presented an exploration into a new context and influences in project practice utilising SSM as the action learning methodology ( $3^{\text {rd }}$ quadrant), a new approach, but not a new system or tool ( $4^{\text {th }}$ quadrant) has been defined.

Given the complex and dynamic nature of the global world, generalizations about patterns of organizational change are difficult "...to sustain over time, and they are even tougher to uphold across international institutional, and cultural borders" (Pettigrew, Woodman et al. 2001b p.697). As the socio-cultural make-up of the UAE changes and acceptable behavioural norms evolve, the findings from the research described in this dissertation and the applicability to a specific context will also change. Unconscious processes of incremental adaptation where being in the world is tempered with a becoming ontology, involve iterative cycles of emergent cognition to underpin learning for organisational change and resilience. Emergence as the reality of complex adaptive systems, assumes contexts evolve and applicability of findings are dynamic.

The relevance of the findings as specific to the research project organisation may therefore shift in time as those in leadership positions come and go, project teams form, evolve and fold and sociopolitical dynamics change on an organisational and national basis. Therefore, caution in generalising findings to other socio-cultural contexts needs to be called for.

Problem owners' world constructs provided a snapshot of the problematical situation from a variety of perspectives over a specific period of time. The transient nature and cultural mix of employees inevitably affect the 'whole' through movements in interconnections. This reality adds to the non- 
static nature of the situation with research results being better viewed as broadening the conceptual base and scope of project practice to reveal the integral nature of project context to project strategy. As noted by Van de Ven (1986), an "...inventory of ideas is seldom adequate for the situation" (p.594), and despite the rigorous nature of SSM it is possible that not all stakeholder perspectives may have been represented accurately. Research involving more formal exploration of departmental members' perceptions of the problematical situation rather than focussing on management team members' perspectives, may have provided a wider range of possible contributing factors.

My role as researcher as embedded in the project context involved a duality in roles from which a degree of subjectivity in interpretation could have been present. Although it is felt that through incorporating development of rich pictures which drew upon problem owners' perceptions, together with detailing the qualitative data from the interviews, researcher subjectiveness was limited.

The inability to implement the recommended actions after the $3^{\text {rd }}$ iterative action cycle due to sudden cessation of my employ at the organisation, limited the ability to evaluate the effectiveness of strategies suggested. Recommendations were further discussed in a more informal $4^{\text {th }}$ cycle, but opportunity to implement, evaluate and trial new actions for organisational transformation was not possible.

\subsection{Recommendations}

The research described in this dissertation has explored a perceived problematical situation that appeared to warrant intervention to bring about improvement. The research has identified the critical nature of human socio-cultural complexity to project practice.

When complexity exists, the project manager cannot have full knowledge on how to master the situation. Rather, emergent knowledge from reflection and action should be wisely drawn upon, not in an attempt to control and determine the outcome, "...but rather cultivate a growth by providing the appropriate environment ..." (von Hayek 1974 p.6). It is recommended that further research into the feasibility of creating an 'appropriate environment' or project culture to facilitate the human interconnections necessary for knowledge creation for optimal learning is a reality. The research in this dissertation has concluded that project practice is more likely to benefit from project strategy that involves an adaptive PM response in context, thus the nature of the response may also benefit from further investigation.

The issue of organisational and nation-state maturity as relevant to project practice and organisational effectiveness emerged from the research findings. Further research to explore the 
significance of contextual maturity may have potential benefits for project management, when attempting to define appropriate responses given the contextual nature of project practice.

The research in this dissertation was implemented in one socio-cultural setting and whilst findings broadened the understanding of the relationship between project practice and the environment, project theory could be further expanded upon through collaborative efforts involving social scientists, project management practitioners and management scholars. SSM as a relevant methodology to pursue further enquiry is recommended, with it being suggested that problematical situations involving project contexts and project teams comprising a disparity in social actors, provide a potentially fertile context to actively seek new and alternative solutions to project management.

As suggested by Folke (2006), "Optimal management will often, because of the complex dynamics, be extremely difficult if not impossible to implement" (p.261), but this does not mean that investigating the ecology of project practice will lack the potential to improve on project management practice or expand the theoretical base and understanding of project actuality. We need to move beyond the traditional approaches of project management that focus on the instrumental nature of project goals and methods (Turner and Cochrane 1993), to view projects as social process, with all the inherent complexities that human plurality creates. New ways of thinking and conceptualising projects given the increasingly ambiguous and uncertain era we face can be supported through researching the comparative merits of acknowledging the autopoietic nature of the project entity and the interface between project and environment.

\subsection{Conclusion}

The aims in the research described in this dissertation have been:

\section{Aim 1:}

To explore the significance of socio-cultural context, socio-technical infrastructure and human capital elements relevant to managing projects for effective change

\section{Aim 2:}

To develop a project management technique or strategy which can:

i. accommodate culturally disparate human capital to,

ii. facilitate communicative action and knowledge transfer to,

iii. promote sense-making for purposeful activity and improved organizational capability 
As introduced in section 1.2, the research in this Project Management Doctoral dissertation aimed to focus on the $3^{\text {rd }}$ quadrant of the DPM practice to theory framework to study 'a new context and influences in project management through action learning' as is reflected in Aim 1. It was also suggested in 1.2 that this research would work towards creating an awareness of new approaches to project management as specific to the $4^{\text {th }}$ quadrant. Whilst the research is perceived as fulfilling Aim 1 , the feasibility in developing new project management systems or tools emerged as typifying more positivist models of project management. Flexible solutions to accommodate for the changing nature of project actuality have been concluded as being more relevant, given the complex nature of the project as social process and the influence of the environmental context. Thus, Aim 2 in part has been achieved in as much as the importance of project strategy and the response of the project manager to balance the dynamics of socially complex project practice has been identified as being critical to project success. The research findings support the notion that a 'one-size-fits-all' strategy to project management is not conducive to positive outcomes due to negating the project's position within a specific environmental context (Cicmil, Williams et al. 2006; Artto, Kujala et al. 2008). This realisation thus influenced a shift away from attempts to define specific new techniques or tools to accommodate for socio-cultural disparities existing in project practice, to focus on exploring feasible PM approaches given the significance of the context.

Selecting Soft Systems Methodology (SSM) as the process of enquiry into the perceived problematical situation, revealed the suitability of its action methodology in contributing to the cognitive enactment underpinning organisational transformational change, through supporting coordinations of stakeholder actions in consensual domains in project practice (Maturana 2002). The process of SSM has been seen as promoting the bringing forth of new worlds of understanding despite social-cultural differences between project actors, helping to sift through 'fuzzy' situations to explore individual real-world constructs which evolved from facilitated interconnections (Dodouras and James 2007). Analytical processes throughout the research described in this dissertation have been supported through defining an integrated research and philosophical conceptual framework to investigate the perceived socially complex nature of project praxis, incorporating enactivist and postmodern thinking in interpretation. 'Projects' in this research have been perceived as messy, cyclic, non-linear and in a state of flux and emergence, with project actualities being identified as complex human activity systems involving dynamic social processes of intertwined human multiple realities. Project actuality is presented as benefitting from appreciating the relevance of complexity theory in visualising the dynamics and evolving nature of project ecologies (Söderlund 2004b; CookeDavies, Cicmil et al. 2007), where contextual and environmental factors cannot be ignored.

The project management literature has noted the need to broaden the research conceptual base for project theory and practice, identifying a number of 'new' areas warranting exploration for relevance 
(Winter, Smith et al. 2006). In reviewing the evidence in this research as presented and discussed in this dissertation, it is felt that concepts presented and conclusions drawn have contributed to:

- Moving towards conceptualising the nature of projects as social process (Winter, Smith et al. 2006);

- Broadening the scope of project research by focussing on the project entity as process rather than research, to appreciate the closer links projects have with their environment as 'project ecologies' (Söderlund 2004b);

- Demonstrating the significance of the embedded nature of the projects in a social context and the associated dynamics (Söderlund 2004a);

- Confirming the complex nature of projects as created by disparate social mixes of people (Cooke-Davies, Cicmil et al. 2007);

- Forming “...a closer link between the practical knowledge and the learning processes that individuals go through in their development as project managers" (Cicmil 2006 p.36);

- Providing an 'alternative lens' to conceptualise the actuality of projects through defining a research and philosophical framework to facilitate new 'insights' into project praxis (Cicmil, Williams et al. 2006);

- Defining a new paradigm for complex projects (Williams 1999).

Findings in this research have revealed a socio-cultural project complexity of a human kind which when viewed assuming a pragmatic epistemology and becoming ontology strengthen the appreciation of the need for strategic project management responses if learning and change is to be effected for organisational resilience.

The socio-cultural complexity of the project environment benefitted from conceptualising the project as a complex adaptive system that draws upon human interconnections in practice for the emerging cognition necessary for adaptive responses. The learning that results from enaction and structurally coupled relationships between actors and the environment, move adaptive behaviour beyond 'being' as evidence of self-regulation, to an ontology of 'becoming' where emergent states evolve. Perceiving the project organisation as autopoietic in nature (Parboteeah and Jackson 2007) supports the notion of the project entity as providing the structural openness for the organisationally closed 'parent' entity. Structurally coupled relationships between individual components within the project entity and the environmental context provide the means for sensing and creating change through relationships based on mutual reciprocity. Organisational resilience is thus seen to be an outcome of cognitive emergence that is facilitated by a multiplicity of interconnections in project praxis embedded in a global ecologically interconnected environment. The cultural mix of the expatriate 
workforce within the Arabic governed research organisation, provided a fertile context in which to reconceptualise the critical nature of the human element to the actuality of project praxis. The challenge was to explore appropriate responses to accommodate for difference to optimise the cognitive enactment that underpins learning.

The research process throughout the two years of inquiry built upon personal and professional experiences initially encountered on arrival in the UAE in 2001. Being immersed in a socio-cultural setting so different in nature from one's own, created new challenges and frustrations on a daily basis. However, it was not so much the differences encountered in 'ways of doing' that necessitated a re-think on what had previously been standard operational practices 'back home', but it was quickly apparent that 'ways of thinking' varied vastly. The socio-political mosaic created by a diverse and fluid expatriate human element embedded in an Arabic system of governance required shifts in communication styles and demanded a sensitivity to the nature of human difference. Experience working with multicultural work teams was not new to the author of this dissertation. What was, however, was the constancy in changing directives in the apparent absence of established or at least acknowledged, processes and policies to guide daily business practice. There were attempts at strategic planning both at departmental and organisational levels. But the focus and emphasis in business operations was on demonstrating the achievement of best possible outcomes in the shortest time-frame possible. Process was not the priority, outcomes kept shifting, and so too organisational priorities, thus creating messy and at times chaotic situations in which to work and find sense. Consequently, management style for survival within the organisation, needed to be flexible, alert to the multi-layered political dynamics and adaptable to local demands.

The ability to compromise and negotiate underpinned an adaptive response to situational circumstances which changed as frequently as the local sands. Identifying the problematical situation described in this dissertation as necessitating some semblance of improvement, was the easiest step in the whole research process. Losing support for what was attempting to be achieved in the project and failing to self-preserve positional authority, were consequences of a political system that saw threat in difference and enquiry, rather than opportunity in identifying barriers and hindrances for successful outcomes. Issues of control were paramount in the research organisation, and an individual inquiry which had not been instigated by the central administrative system despite being approved by the college directorship, was perceived as potentially damaging to reputation. Nevertheless, learning for improvement through working with stakeholders, reflecting on practice and making recommendations for change, were overall positive outcomes that underpinned the recommended project manager's response as represented in Figure 35. Further research into the interrelationships of power, culture and change as experienced throughout this research, potentially may unravel further some of the complexities inherent to project praxis. 
Thus the emergent nature of cognition enacted through dynamic multiple interrelationships as noted in the research project organisation may assume learning is a product and enabler of transformative and adaptive change (Reid 1996). As work boundaries shift and project practice increasingly becomes the new work reality, calls for "...coherent new theoretical frameworks that attempt to overcome the aged dichotomies between micro-and macro-, mental and material, quantitative and qualitative, observation and intervention" (Engeström 2000 p.961) are being made. Effective project management necessitates front-end recognition of the central nature of the human essence in project processes, with this research identifying the complexity created by socio-cultural differences and the imperative to facilitate some semblance of consensus action for positive project outcomes.

In an ideal world Boulding (2000) suggests a "... mosaic of identities, attitudes, values, beliefs and institutional patterns .... [can] ... lead people to live nurturantly with one another, and the earth itself without the aid of structured power differentials, to deal creatively with their differences and share their resources" (p.196). This unfortunately is not a reality in everyday life nor project praxis. The embedded nature of structural power inequalities residing within structure-agency interconnections underpin the ongoing tensions that arise from conflicting systems of autonomy and control. The powerful interplays that are a constant in project practice will not benefit from attempts to resist or compete, rather strategic approaches are needed to balance the situational dynamics involving team members, contextual politics and socio-cultural differences in understanding. The PM response does not 'sit outside' the project entity or organisation, rather the PM is embedded in context, being influenced and in turn influencing the environment due to the ecological nature of interconnected realities. The PM, despite the existence of contextual constraints, is in a leadership position with 'power to' effect the interconnections of team players to facilitate emergent cognition in project practice. Successful or positive outcomes however, are dependent upon the PM's ability to combine this 'power to' effect change with responses that are adaptive, flexible and 'profoundly conscious'. That is, the findings in this research suggest that the socially complex realities existing as today's project actuality demand a profound PM response, where as leader s/he may be seen to have and apply the "...intellectual insight that extends far beyond the boundaries of common knowledge" (Edgeman and Fraley 2008 p.683). Complex adaptive systems call for complex adaptive responses to circumnavigate the detrimental elements arising from power games that can hinder emergent cognition integral to adaptive change. Learning to manage this change needs to move beyond systems of governance by control if an ontology of becoming is to be seen in systems of resilience (Folke 2006). A profoundly conscious PM has the potential to facilitate the collective consciousness necessary for project action through fostering human interconnections that despite cultural differences, can result in the emergence of new world constructs and consensus action. 
Findings in this research suggest that a focus on strategic action needs to consume more time in project practice than lengthy sessions on strategic planning, vision building and attempts to seek agreement on project paths or in defining linear courses. We are in an era of uncertainty and ambiguity that demands incremental project strategy where process is emergent and contextually relevant for positive outcomes and effective change. Complexity of the human kind, not just technical, is the project actuality needing to be addressed, with inextricable links in social processes as specific to an environmental context being the new reality. The critical nature of project management will benefit from a shift in focus from instrumental approaches in project research and practice, to viewing the ecological nature of projects where a multiplicity of world realities connect and collide. Individuals being enabled to find meaning through sensemaking activities in project practice for emergent cognition and knowledge creation can shift an organisational ontology of stable adaptive being to an emergent perpetually evolving state of becoming. The balance between these ontological states is more assured if the PM response is adaptive, moving beyond selfregulatory behaviour aimed at maintaining stability, to promoting the human interconnections and action which underpin knowledge creation for learning, change and resilience (Figure 35).

It is not presupposed that there is 'one' finite theory or strategic approach to manage complex projects, as this would negate the main thesis in this research dissertation. Rather a change in focus in the actuality of projects is recommended so an appreciation of the critical interconnected nature of the human element in projects as social process and the inextricable links project practice has in context may be gained. Project strategy thus needs to assume a PM response which is flexible and in tune with the environment, to support the human-structure interconnect which not only exists within the organisational context, but within a larger socio-political and in turn global context. Accepting this reality together with the constancy found in change, helps shifts the focus of projects as instrumental processes to projects as social processes that entail complex dynamics warranting profound and skilful management. Through balancing such dynamics, the PM response has the potential to bring forth new worlds of meaning in project practice, optimising the value found in acknowledging the realities of new and emergent project ecologies. 


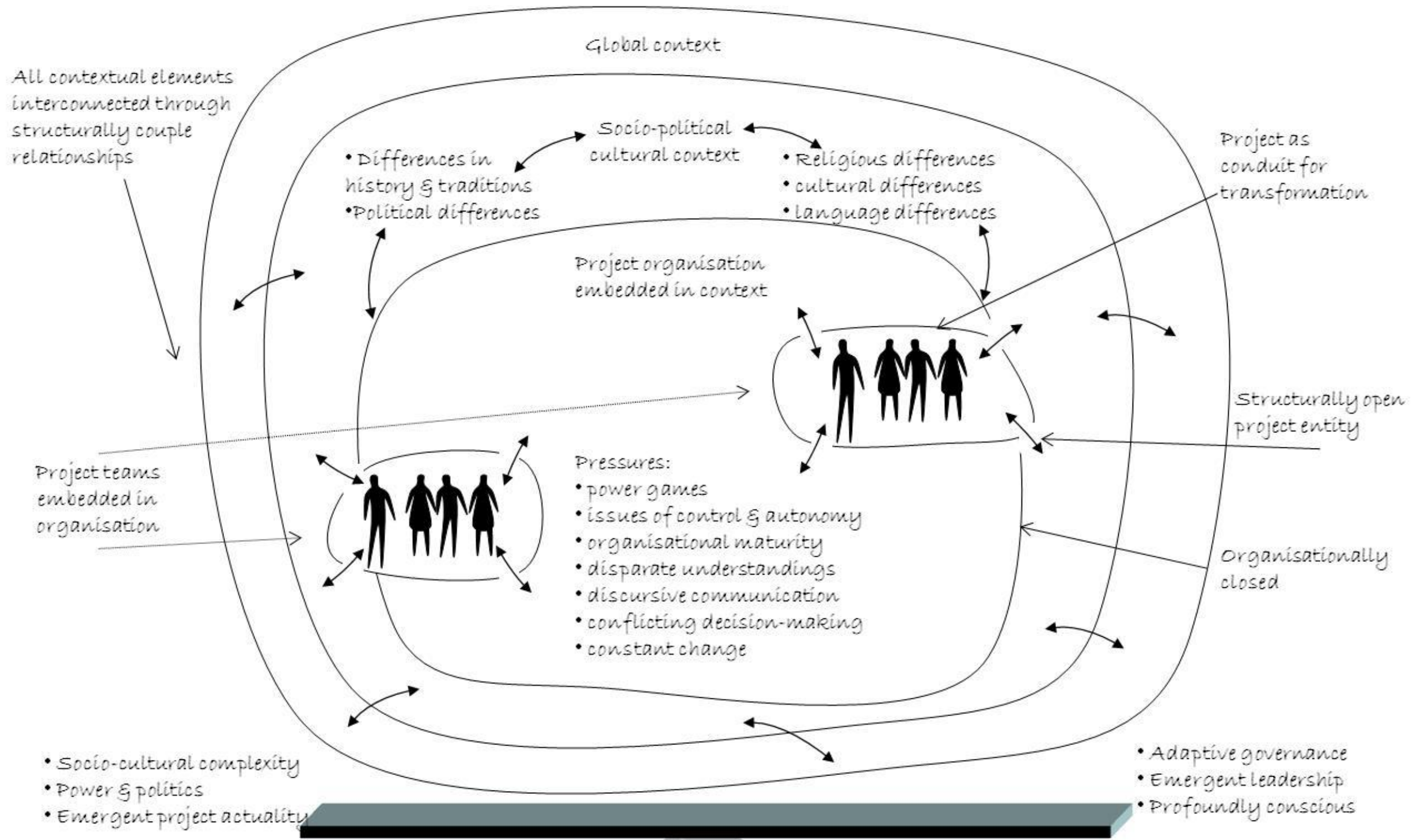




\section{Glossary of Terms}

A(ctors)

allopoiesis

assabiyah

autonomy

autopoiesis

Autopoietic nature

of learning

organisation,

resilience, emerging

ontologies \& project

ecologies
'Actors' - defined in SSM as those who can make the transformation process ( $T$ ) happen through being involved in the activities necessary; these may also be 'owners of the issue to be addressed' i.e. those concerned or affected by the situation enough to want to bring about an improvement. This is different from 'owner' as 'client' who caused the intervention to happen. In this dissertation 'actors' and 'problem or issue owners' are identified as individuals who can make T happen

Process by which system produces something other than itself. Opposite to autopoiesis

Arabic term for sense of group consciousness or collective mind (Khaldun 2005)

In relation to enactivist thought on living systems, autonomy is a characteristic that assumes ability to self-produce for adaptability and survival, as in the process of autopoiesis

'auto' means 'self' and refers to the autonomy of self-organizing systems; and poiesis - which shares the same Greek root as the word 'poetry' - means 'making'. i.e. autopoiesis means 'self-making'” (Capra 1997). That is, autopoietic systems are continually emergent in nature, creating and adapting to stimuli/perturbations in the environment due to interconnections and dynamic networked relationships that all components have with each other in context. Interactions involve mutual reciprocity that ensures the system continues to adapt and emerge for survival, whilst influencing change within the environment due to being structurally coupled and embedded within a context. Living systems as autopoietic 'act' to adjust, necessitating cognitive processes that arise through the connect of language, modifying self, the entity and the overall environmental space, whilst ensuring a degree of stability for survival.

See following papers for further reading on the learning organistion as autopoietic and related enactivist cognitive theory:

Baerveldt, C. and T. Verheggen (1999). "Enactivism and the Experiential Reality of Culture: Rethinking the Epistemological Basis of Cultural Psychology." Culture Psychology 5(2): 183-206.

Folke, C. (2006). "Resilience: The emergence of a perspective for social-ecological systems analyses." Global Environmental Change 16(3): 253-267.

Hall, W. P. (2005). "Biological nature of knowledge in the learning organisation." The Learning Organisation 12(2): 169-188.

Lineham, C. and D. Kavanagh (2006). From project ontologies to communities of virtue. Making projects critical. D. Hodgson and S. Cicmil. New York, Palgrave Macmillan: 51-67.

Parboteeah, P. and T. W. Jackson (2007). "An Autopoietic Framework for Organisational Learning." Knowledge and process management 14(4): 248-259. 
Proulx, J. (2008). "Some Differences between Maturana and Varela's Theory of Cognition and Constructivism." Complicity: An International Journal of Complexity and Education 5(1): 11-26.

Söderlund, J. (2004b). "On the broadening scope of the research on projects: a review and a model for analysis." International Journal of Project Management 22: $655-667$.

Varela, F., H. Maturana, et al. (1974). "Autopoiesis: The organization of living systems, its characterization and a model." BioSystems 5: 187-196.

Chaotic

Client

CATWOE

Co-emergence

Cognition

Consensual domains

DAT

Din

E's (the $3 E^{\prime} s$ )
Non-random, patterned, complex, deterministic behaviour (Capra 1997); contrasts with chaos, which is seen as erratic and random

'Client' in SSM as identified in Analysis 1 - those who had caused the intervention to happen; their role may overlap ' $O$ ' as owners who could stop the transformation process

Mnemonic representing a generic model of purposeful activity as defined in SSM. Where $\mathrm{C}=$ Customers are victims or beneficiaries affected by the $\mathrm{T}=$ Transformation process; $\mathrm{A}=$ actors who are involved in the activity of $\mathrm{T} ; \mathrm{W}=$ Weltanschauung or worldview usually of $\mathrm{C} ; \mathrm{O}=$ owners who could stop the transformation process (this could be Customers as 'issue' or 'problem owners', 'Actors' or the 'Client' as identified in analysis 1 of SSM as the one who had caused the intervention to happen in the first instance); $E=$ Environmental constraints which may limit the transformation process (Checkland and Poulter 2006).

Refers to the changing nature of both the living system and the context; resulting from relationships based on mutual reciprocity where interconnections affect both the system and the environment, with emerging states of all components supporting the notion of a becoming ontology

Ecological or enactivist view sees cognition as a process where bringing forth of a world arises from the process of living where individuals are interconnected cognitively through language and with the environment; perception and action are inseparable. That is, cognition is embodied with capabilities emerging from social interactions between components and the environment.

Social interactions between autonomous systems take place through coordinations of behaviour within domains that can be seen as 'co-operative' (Maturana 1978; Maturana 1980; Maturana and Varela 1980; Baerveldt and Verheggen 1999). The praxis of language takes place in consensual domains whereby new worlds of reality are brought forth through social interactions.

Divisional Academic Team

Arabic word meaning religion - as defining a group collectivity

Efficacy - 'criteria to tell whether the transformation is working';

Efficiency - 'criteria to tell whether the transformation is being achieved';

Effectiveness - 'criteria to tell whether this transformation is helping achieve some higher-level or longer term aim' (Checkland and Poulter 2006 p.42)

The flow of emotions (Maturana 1998) 
Enactivism

E (nvironmental)

Fatwas

Fiqh

GO

Hadith

Interobjectivity

$\mathrm{KC}(\mathrm{A})$

Knotworking

Language

Languaging
A theory of cognition wherein '....a cognitive system operates as a producer of meaning rather than as a processor of information...' (Baerveldt and Verheggen 1999 p.195); where social interaction between organisationally closed but structurally open autopoietic living systems is enabled through structurally coupled relationships between interacting autonomous systems and the environment. Embodied experience and patterns of social relatedness determine the emergent nature of knowledge, where action and communication interrelate to bring forth a world of meaning (Gergen 1985; Varela, Thompson et al. 1991; Reid 1996; Maturana 2002). Enactivism views reality as emerging through consensual co-ordinations of behaviour.

'Environmental' factors in SSM which may hinder or constrain action necessary for Transformation $(T)$ processes

Islamic authoritative legal opinions are drawn from

Islamic jurisprudence, the interpretive arm of Arabic law and legislation

Graduate Outcome

Narrations of Prophet Muhammad's and first Muslims' sayings and stories

A world of meaning emerges from a junction that exists between the physical world and self, where there is a '...continual back-and-forth influence of knower and known...' through structurally coupled relationships (Proulx 2008 p.23). It is the concept of interobjectivity that underpins the emergent nature of a becoming ontology where a world of new realities are brought forth from interconnections that negate the concept of a structure-agent disconnect

Key Criteria (Assessment)

A word coined by Engeström (2006) to describe dynamic, collaborative processes that aimed to connect actors in team work efforts

Maturana's (1995) biological definition 'a manner of flowing in living together in recursive consensual co-ordinations of behaviour'. Enactivist view of language in relation to living systems, sees language emerging through individual components of a system (i.e. those with a nervous system) from being inextricably linked with others within a context - i.e. structurally coupled where "...self-consciousness arises as an individual phenomenon from the recursive structural coupling of an organism with language with its own structure through recursive self-description" (Maturana 1999).

'...consists in operating in a network of consensual co-ordinations of consensual co-ordinations of behaviors' (Maturana 1995 p.1); It is thus languaging which underpins human complexity that in turn brings forth a world of meaning through interconnections, where consciousness is co-emergent. As quoted by Maturana (1988) "We kill or elate with words because, as co-ordinations of actions, they take place through body interactions that trigger in us body changes in the domain of physiology" (p.48) 
O (wners)

Majilis

Phronesis

PQR

Praxis

Problem owners

Qur'an

Resilience

Root Definition

Self-organisation

Sensemaking

Shari'a

Situated cognition
'Owners' in SSM are those who could stop the transformation process. This may also include or overlap with roles of Actors who can do the activities to make the transformation happen or 'issue owners' as identified by the practitioner or problem owner who initiated the investigation. The 'Client' who caused the investigation in the first place could also be seen to act as an 'Owner'. i.e. multiple and overlapping roles may exist

Arabic meeting and meeting place; A place where a ruler may receive complaints, and others who attend or are present may give their view (HeardBey 2004)

Originally drawn from Aristotle's concept of 'practical wisdom' or virtue of moral thought (Thomson 1955)

A formula used in SSM to create a Root Definition, where $P=$ the 'what' $Q=$ 'how' and $R=$ ' why'. Defining the PQR results in the $Q$ representing the transformation process

A form of action that is fundamentally contingent on context-dependent judgment and situational ethics (Cicmil 2006)

'problem owner' as defined in SSM is the practitioner who identified a problematical situation considered worthy of exploration to seek improvement. In the research described by this dissertation 'problem owners' is the term used for those 'issue owners' identified by myself as practitioner. I saw myself as a 'problem owner' but so too the 'issue owners' were 'problem owners' as their worldview conceded that there was a problematical situation needing action for improvement

Islamic sacred book of Prophet Muhammad's revelations from Allah

“...the capacity for renewal, re-organization and development" (Folke 2006 p.253)

Term used in SSM which represents a statement that "... described the purposeful activity being modelled as a transformation process..."(Checkland and Poulter 2006 p.39)

'...the spontaneous emergence of new structures and new forms of behaviour in open systems far from equilibrium, characterised by internal feedback loops and described mathematically by nonlinear equations" (Capra 1997). A quality of autopoietic living systems, underpinning adaptation, stability and survival.

Gaining an understanding or ascribing meaning to a situation or event; associated with embodied cognition where individual world constructs arise from consensual experience through language and social interaction

The dominant system of Islamic law based on the texts of the Qur'an and Sunnah

Biological or ecological view assumes what has been defined as 'innate' behaviour and 'learned' behaviour are indistinguishable due to the embodied nature of cognition removing any object-subject divide. Object and subject 
merge, no ultimate 'truth' is 'out there', cognition emerges through individuals co-existing and participating in a physical world where knowledge is enacted to bring forth worlds of meaning through interobjective relationships (Proulx 2008)

Structural coupling Exists 'whenever there is a history of recurrent congruence between two (or more) systems' (Maturana and Varela 1998); structural connections between entities and with the environment involving an 'interobjectivity'; relationship assures survival through adaptation and response to environmental perturbations that may be selective - that is the environment does not determine the change, rather acts as a trigger to entities within which structurally coupled relationships exist

Sunnah Muslim way of life based on the life and teachings of Prophet Muhammad

Weltanschauung

German derived word meaning 'worldview'; in SSM weltanschauung refers to how individuals perceive and view the world from their own reality or construct. Thus worldviews may clash or perhaps display external similarities in articulation, but in essence a person's weltanschauung is unique and differences need to be accommodated for. 


\section{References}

Abernathy, M. A., Vagnoni, E., (2004). "Power, organization design and managerial behaviour." Accounting, Organizations and Society 29: 207-225.

Agostinho, S. (2004). "Naturalistic inquiry in e-learning research." International Journal of Qualitative methods 4(1 March 2005): Retrieved Nov 2008

http://www.ualberta.ca/ iiqm/backissues/4 1/pdf/agostinho.pdf.

Al Hashimi, M. A. (1996). The Muslim women and her Rabb: The ideal Muslimah. Ontario, International Islamic Publishing House.

Aligica, P. D. (2006). "Institutional and Stakeholder Mapping: Frameworks for Policy Analysis and Institutional Change." Public Organizational Review 6: 79-90.

Allen, P. M. (2006). "Editorial - Complexity." Emergence: Complexity and Organization 8(1): iv-v.

Amabile, T. M. (1998). "How to kill creativity." Harvard Business Review Sept-Oct: 76-87.

Ambos, B. and B. Schlegelmilch (2008). "Innovation in Multinational Firms: Does Cultural Fit Enhance Performance?" Management International Review 48(2): 189-206.

Antonakis, J., Avolio, B.J., Sivasubramaniam, N. (2003). "Context and leadership: an examination of the nine-factor full-range leadership theory using the Multifactor Leadership Questionnaire." The Leadership Quarterly 14(3): 261-295.

APM. (2008-9). "Portfolio, Programme, and Project Management Maturity Model (P3M3)." Retrieved 20th January, 2008, from http://www.p3m3-officialsite.com/home/home.asp.

Argyris, C. (1999). On Organizational learning. United Kingdom, Blackwell Publishing.

Argyris, C. and D. Schön (1996). Organisational Learning II: Theory, method and practice. USA, Addison-Wesley.

Artto, K., J. Kujala, et al. (2008). "What is project strategy?" International Journal of Project Management 26(1): 4-12.

Ashkanasy, N., E. Trevor-Roberts, et al. (2002). "The Anglo Cluster: legacy of the British empire." Journal of World Business 37: 28-39.

Atkinson, R., L. Crawford, et al. (2006). "Fundamental uncertainties in projects and the scope of project management." International Journal of Project Management 24: 687-698.

Atwater, D. C. and B. M. Bass (1994). Transformational Leadership in Teams. Improving Organizational Effectiveness Through Transformational Leadership. B. M. Bass and B. J. Avolio. California, Sage.

Avolio, B. J., W. L. Gardner, et al. (2004). "Unlocking the mask: A look at the process by which authentic leaders impact follower attitudes and behaviours." The Leadership Quarterly 15: 801-823.

Babbie, E. (1998). The practice of social research (8th edition) Belmont, CA, Wadsworth. 
Baerveldt, C. and T. Verheggen (1997). Toward a psychological study of culture: epistemological considerations. 7th conference of the International Society for Theoretical Psychology (ISTP), Berlin http://members.shaw.ca/ncpg/baerveldt verheggen.html.

Baerveldt, C. and T. Verheggen (1999). "Enactivism and the Experiential Reality of Culture: Rethinking the Epistemological Basis of Cultural Psychology." Culture Psychology 5(2): 183-206.

Barber, M. (2002). "Alfred Schutz." Winter. from http://plato.stanford.edu/archives/win2002/entries/schutz/.

Barkema, H. G., O. Shenkar, et al. (1997). "Working abroad, working with others: How firms learn to operate international joint ventures." The Academy of Management Journal 40(2): 426-442.

Barnard, C. I. (1938). The Functions of the Executive. Cambridge, MA, Harvard University Press.

Barnett, R. (2004). "Learning for an unknown future." Higher Education \& Research 23(3): 247-260.

Barney, J. (1991). "Firm resources and Sustained Competitive Advantage." Journal of Management 17(1): 99-120.

Bartunek, J., J. Trullen, et al. (2007). "Front and Backstages of the Diminshed Routinization of Innovations: What Innovation Research makes Public and Organizational Research Finds Behind the Scenes." Strategic Entrepreneurship Journla 1: 295-314.

Bass, B. M. (1960). Leadership, psychology and organizational behavior. New York, Harper.

Bass, B. M. and B. J. Avolio (1994). Improving organizational effectiveness, through transformational leadership. California, Sage Publications.

Bass, B. M. and P. Steidlmeier (1999). "Ethics, character and authentic transformational leadership behaviour." The Leadership Quarterly 10(2): 181-217.

Bateman, D. (2006). Doing futures: Futures education and enactivism. Australian Teacher Association Education Conference, Fremantle, WA, Australia.

Bateson, G. (1979). Mind and Nature: A necessary unity. New York, Dutton.

Bateson, G. (1987). Steps to an ecology of the mind. North Vale NH, Jason Aronson.

Bausch, K. (2008a). "The Ethics of Emergence " Systems Research and Behavioral Science 25: 271276.

Bausch, K. (2008b). "Practical ethics for group decisions in complex situations." Systems Research and Behavioral Science 25(2): 277-281.

Beer, S. (1981). The Brain of the Firm: The Managerial Cybernetics of Organisation. Chichester, Wiley \& Sons.

Begg, A. (2002). "Enactivism and some implications for education: A personal perspective." Vinculum 39(2): 4-12.

Benner, P. (1984). From novice to expert: excellence and power in clinical nursing practice. Menlo Park CA, Addison-Wesley. 
Bennis, W. and B. Nanus (2003). Leaders: Strategies for Taking Charge. NY, Harper Collins.

Berger, J. (1977). Ways of Seeing. London, Penguin Books.

Berger, P. and T. Luckman (1967). The Social Construction of Reality: A Treatise in the Sociology of Knowledge. New York, Double Day.

Bergvall-Kåreborn, B. (2002). "Enriching the Model-Building Phase of Soft Systems Methodology." Systems Research and Behavioral Science 19: 27-48.

Bhagat, R. S., B. L. Kedia, et al. (2002). "Cultural variations in the cross-border transfer of organizational knowledge : An integrative framework." Academy of Management Review 27(2): 204221.

Bhatt, G. D. (2000). "Information dynamics, learning and knowledge creation in organizations." The Learning Organization 7(2): 89-98.

Bhatt, G. D. (2001). "Knowledge management in organizations: examining the interaction between technologies, techniques and people." Journal of Knowledge Management 5(1): 68-75.

Bilgin, P. (2006). "What future for Middle Eastern studies?" Futures 38: 575-585.

Bloch, D. P. (2005). "Complexity, Chaos, and Nonlinear Dynamics: A New Perspective on Career Development Theory." The Career Development Quarterly 53: 194-207.

Bloom, B. S., M. D. Engelhart, et al. (1956). Taxonomy of Educational Objectives: The Classification of Educational Goals: Handbook 1 Cognitive Domain. New York, McKay.

Boddy, D. and R. Paton (2004). "Responding to competing narratives: lessons for project managers." International Journal of Project Management 22: 225-233.

Bohm, D. (1992). Thought as a system. London, Routledge.

Boisvert, R. (1998). John Dewey: rethinking our time. Albany NY, SUNY.

Bonney, R. (2004). "Reflections on the Differences Between Religion and Culture." Clinical Cornerstone 6(1): 25-33.

Boreham, N. and C. Morgan (2004). "A sociocultural analysis of organisational learning." Oxford Review of Education 30(3).

Bottery, M. (2006a). "Education and globalization: redefining the role of the educational professional." Educational Review 58(1): 95-113.

Boulding, E. (2000). "A new chance for human peacefulness? Peace and Conflict." Journal of Peace Psychology 6: 193-215.

Bourdieu, P. (1977). Outline of a Theory of Practice. Cambridge, Cambridge University Press.

Bourdieu, P. (1996). The rules of art: genesis and structure of the literary field. Cambridge, Polity Press.

Bourdieu, P. (1998). Practical Reason: On the Theory of Action. Stanford, California, Stanford University Press. 
Bourne, L. and D. Walker (2005). Stakeholder Chamelon - Ignore at your Peril! PMI Global Congress Singapore.

Bredillet, C. (2008). "Learning and acting in project situations through meta-method (MAP) a case study: Contextual and situational approach for project management governance in management education." International Journal of Project Management 26: 238-250.

Brennan, A., Dooley, L. (2005). "Networked creativity: a structured management framework for stimulating innovation." Technovation 25(12): 1388-1399.

Bresnen, M. (2006). Conflicting and conflated discourses? Project management, organisational change and learning. Making Projects Critical. D. Hodgson and S. Cicmil. New York, Palgrave Macmillan: 68-89.

Bresnen, M. (2007). "Deconstructing partnering in project-based organisation: Seven pillars, seven paradoxes and seven deadly sins." International Journal of Project Management 25: 365-374.

Bresnen, M., L. Edelman, et al. (2003). "Social practices and the management of knowledge in project environments." International Journal of Project Management 21: 2003.

Brugha, R. and Z. Varvasovszky (2000). "Stakeholder analysis: a review." Health Policy Plan 15(3): 239-246.

Buchinger, E. (2006). "The sociological concept of autopoiesis: Biological and philosophical basics and governance relevance." Kybernetes 35(3/4): 360-374.

Burden-Leahy, S. (2005). "Addressing the Tensions in a Process-based Quality Assurance Model Through the Introduction of Graduate Outcomes: a case study of the change process in a vocational higher education institution in the United Arab Emirates." Quality in Higher Education 11(2): 129136.

Calori, R. (2002). "Essai: real-time/real-space research: connecting action and reflection in organization studies." Organization Studies 23(6): 877-883.

Campos, E. B. and M. P. S. Sanchez (2003). "Knowledge management in the emerging strategic business process: information, complexity and imagination." Journal of Knowledge Management 7(2): 5-17.

Capra, F. (1983). The Turning Point: Science, Society and the Rising Culture. London, Flamingo.

Capra, F. (1997). The Web of Life: A New Synthesis of Mind and Matter. London, Flamingo.

Carlisle, Y. and E. McMillan (2006). "Innovation in organizations from a complex adaptive systems perspective." Emergence: Complexity and Organization 8(1): 2-9.

Carlopio, J. (1998). Implementation: Making workplace innovation and technical change happen. Sydney, McGraw-Hill.

Cavaleri, S. (2004). "Leveraging organizational learning for knowledge and performance." The Learning Organization 11(2): 159-176.

Cavaleri, S. and D. Fearon (1996). Managing in Organizations that Learn. USA, Blackwell. 
Cavaleri, S. and F. Reed (2000). "Designing Knowledge Creating Processes." Knowledge and Innovation: Journal of the KMCl 1(1): 109-131

http://www.kmci.org/media.cavaleridesigningkcprocess.pdf.

Cavaleri, S. and F. Reed (2001). "Organizational Inquiry: The Search for Effective Knowledge." Knowledge and Innovation: Journal of the KMCl 1(3): 39-57

http://www.kmci.org/media/cavalerireedkiv1n3.pdf.

Cavaleri, S., S. Seivert, et al. (2005). Knowledge Leadership: The Art and Science of the Knowledgebased Organization. Oxford, UK, Elsevier Butterworth-Heinemann.

Chapra, M. U. (2006). Ibn Khaldun's theory of development: Does it help explain the low performance of the present-day muslim world? International Congress of Ibn Khaldun, Madrid, Spain, Univerisdad Nacional de Education a Distance (UNED) http://www.uned.es/congresco-ibnkhaldun/pdf/12\%20Umer\%20Chapra.pdf.

Checkland, P. (1981). Systems Thinking, Systems Practice. Chichester, Wiley.

Checkland, P. (1995). "Model validation in soft systems practice." Systems Research 12(1): 47-54.

Checkland, P. and S. Holwell (1998). "Action Research: Its Nature and Validity." Systemic Practice and Action Research 11(1): 9-21.

Checkland, P. and J. Poulter (2006). Learning for Action. West Sussex, England, John Wiley \& Sons.

Checkland, P. and J. Scholes (1990). Soft Systems Methodology in action. Chichester, West Sussex, John Wiley and Sons.

Checkland, P. and C. Tsouvalis (1997). "Reflecting on SSM: the link between root definitions and conceptual models." Systems Research and Behavioral Science 14: 153-168.

Checkland, P. and M. Winter (2006). "Process and content: two ways of using SSM." Journal of the Operational Research Society 57: 435-444.

Chia, R. (1995). "From Modern to Postmodern Organizational Analysis." Organization Studies 16(4): 579-604.

Choi, B., S. K. Poon, et al. (2008). "Effects of knowledge management strategy on organizational performance: A complementarity theory-based approach." The International Journal of Management Science 36: 235-251.

Choo, A. S., K. W. Linderman, et al. (2007). "Method and context perspectives on learning and knowledge creation in quality management." Journal of Operations Management 25: 918 - 931.

Christis, J. (2005). "Theory and Practice of Soft Systems Methodology: A Performative Contradiction?" Systems Research and Behavioral Science 22: 11-26.

Chuah, S.-H., R. Hoffmann, et al. (2007). "Do cultures clash? Evidence from cross-national ultimatum game experiments." Journal of Economic Behavior \& Organization 64(1): 35-48.

CIA. (2008). "World Factbook - United Arab Emirates - Country Profile 2008." from http://www.indexmundi.com/united arab emirates/. 
Cicmil, S. (2006). "Understanding Project Management Practice Through Interpretative and Critical Research Perspectives." Project Management Journal 37(2): 27-37.

Cicmil, S. and D. Hodgson (2006). Making projects critical: an introduction. Making Projects Critical. D. Hodgson and S. Cicmil. New York, Palgrave Macmillan: 1-25.

Cicmil, S., T. Williams, et al. (2006). "Rethinking Project Management: Researching the actuality of projects." International Journal of Project Management 24: 675-686.

CIDA (1995). Measuring Up or Muddling Through: Best Practice in the Australian Non-Residential Construction Industry. Sydney, Australia, Construction Industry Development Agency and Masters Builders Australia: 59-63.

Cilliers, P. (2000). "What can we learn from a theory of complexity?" Emergence 2(1): 23-33.

Clark, E. and M. Geppert (2006). "Socio-political processes in international management in postsocialist contexts: Knowledge, learning and transnational institution building." Journal of International Management 12(3): 340-357.

Clarke, M. and D. Otaky (2006). "Reflection 'on' and 'in' teacher education in the United Arab Emirates." International Journal of Educational Development 26: 111-122.

Clegg, S. (2006). "The bounds of rationality: Power/history/imagination." Critical perspectives on Accounting 17: 847-863.

Clegg, S. and D. Dunkerley (1980). Organization, Class and Control. London \& Boston, Routlege and Kegan Paul.

Clegg, S. and A. Ross-Smith (2003). "Revising the boundaries: Management education and learning in postpositivist world." Academy of Management Learning and Education 2(1): 85-98.

Clegg, S. R. (1989). Frameworks of Power. London, Sage.

Clegg, S. R., T. S. Pitsis, et al. (2006). Making the future perfect: constructing the Olympic dream. Making Projects Critical. D. Hodgson and S. Cicmil. New York, Palgrave Macmillan: 265-293.

Coghlan, D. (2007a). "Insider action research: opportunities and challenges." Management Research News 30(5): 335-343.

Coghlan, D. (2007b). "Insider action research doctorates: Generating actionable knowledge." Higher Education 54: 292-306.

Coghlan, D. and T. Brannick (2001). Doing Action Research in Your Own Organization. London, Sage.

Cohen, M. D., J. G. March, et al. (1972). "A Garbage Can Model of Organizational Choice." Administrative Science Quarterly 17(1): 1-25.

Cohen, W. M., Levinthal, D.A. (2001). "Absorptive capacity: A new perspective on learning and innovation." Administrative Science Quarterly 35: 128-152.

Cooke-Davies, T. J. and A. Arzymanow (2003). "The maturity of project management in different industries: An investigation into variations between project management models." International Journal of Project Management 21: 471-478. 
Cooke-Davies, T. J., S. Cicmil, et al. (2007). "We're not In Kansas Anymore, Toto: Mapping the Strange landscape of Complexity Theory, and Its Relationship To Project Management." Project Management Journal 38(2): 50-61.

Cooper, R. and G. Burrell (1988). "Modernism, postmodernism and organizational analysis: An introduction." Organization Studies 9(1): 91-112.

Cope, B. and M. Kalantzis (1997). Productive diversity - Culture and citizenship. Productive Diversity: A New Australian Model for Work and Management. Sydney, Australia, Pluto Press.

Córdoba, J.-R. and F. Farquharson (2008). "Enquiring into Skills Development with SSM: A South African Experience." Systems Research and Behavioral Science 25: 81-97.

Courtney, J., B. Chae, et al. (2000). "Developing Inquiring Organizations." Knowledge and Innovation: Journal of the KMCl 1(1): 132-145 http://www.kmci.org/media/courtney.pdf.

Crawford, L., J. Pollack, et al. (2006). "Uncovering the trends in project management: Journal emphases over the last 10 years." International Journal of Project Management 24: 175-184.

Crossan, M. M., H. W. Lane, et al. (1999). "An Organizational Learning Framework: From Intuition to Institution." Academy of Management Review 24(3): 522-537.

Crowther, D. and M. Green (2004). Organizational Sociology. London, CIPD House.

Daft, R. (1983). Organization theory and design. New York, West.

Daft, R. L. and K. E. Weick (2001). Toward a Model of Organizations as Interpretation Systems. Making Sense of the Organization. K. E. Weick. Oxford, UK, Blackwell Publishing: 241-258.

Dahl, R. A. (1957). "The Concept of Power." Behavioral Science 2: 201-215.

Dambrin, C., C. Lambert, et al. (2007). "Control and change - Analysing the process of insitutionalisation." Management Accounting Research doi:10.1016/j.mar.2007.02.003.

Davenport, T. H. and L. Prusak (2000). Working Knowledge: How organizations manage what they know. Harvard, USA, Harvard Business School Press.

Davis, J., Subrahmanian, E., Westerberg, A. (2005). Knowledge Management: Organizational and technological dimensions. New York, Physica-Verlag.

De Fillippi, R. J. (2001). "Introduction: Project-based Learning, Reflective Practices and Learning Outcomes." Management Learning 32(1): 5-10.

De Geus, A. (1997). "The Living Company." Harvard Business Review 75(2): 51-59.

De Long, D. W. and L. Fahey (2000). "Diagnosing cultural barriers to knowledge management." Academy of Management Executive 14(4): 113 - 127.

De Paula, R. (2005). Post-modernity, Values and HCl Assessment. CHI: Values \& Design Workshop, Institute for Software Research, University of California, Irvine.

Deming, W. E. (1994). The New Economics for industry, Education, Government. Cambridge, MA, MIT Press. 
Derrida, J. (1973). Speech and phenomena. Evanston, Northwester University Press.

Derrida, J. (1992). Structure, sign and play. New York, Harcourt Jovanovitch.

Dervitsiotis, K. N. (2008). "Guiding human organisations to climb the spiral stages of performance improvements." Total Quality Management 19(7-8): 709-718.

Descartes, R. (1637). Discourse on the method. Leiden, Ian Maire.

Dewey, J. (1929). The Quest for Certainty: A Study of the Relation of Knowledge and Action. New York, G.P. Putnam.

DiTomaso, N. and R. Hooijberg (1996). "Diversity and the Demands of Leadership." Leadership Quarterly 7(2): 163-187.

Dixon, N. M. (2000). Common Knowledge: How companies thrive by sharing what they know. Boston, Harvard Business School Press.

Dodouras, S. and P. James (2007). "Fuzzy cognitive mapping to appraise complex situations." Journal of Environmental Planning and Management 50(6): 823 - 852.

Dooley, K. (1996). "A Nominal Definition of Complex Adaptive Systems." Chaos Network 8(1): 2-3.

Dowding, K. (2008). "Agency and structure: Interpreting power relationships." Journal of Power 1(1): 21-36.

Dreyfus, H. L. (1986). Mind over Machine: the power of human intuition and expertise in the age of the computer. Oxford, Basil Blackwell.

Drucker, P. F. (1967). The Effective Executive: The definitive guide to getting the right things done. New York, Harper Collins.

Drucker, P. F. (1986). The Frontiers of Management. Oxford, Butterworth-Heinemann.

Edgeman, R. L. and L. A. Fraley (2008). "A system of Profound Consciousness: Building beyond Deming." Total Quality Management 19(7-8): 683-707.

Edkins, J. (1999). Poststructuralism and International Relations: Bringing the Political Back. London, Rienner Publishers.

Eijnatten, F. M. (2004). "Chaordic systems thinking: Some suggestions for a complexity framework to inform a learning organization." The Learning Organization 11(6): 430-449.

Ekbia, H. and R. Kling (2003). "Power Issues in Knowledge Management." The Internet and Unrefereed Scholarly Publishing WP-03-02: 1-18.

Ekinsmyth, C. (2002). "Project organization, embeddedness and risk in magazine publishing." Regional Studies 36(3): 229-243.

El-Bizri, N. (2003). Avicenna's De Anima between Aristotle and Husserl. The Passions of the Soul in the Metamorphosis of Becoming. A. T. Tymieniecka. Dordrecht, Kluwer Academic Publishers: 67-89.

Emden, C. J. (2005). Nietzsche on Language, Consciousness and the Body. Urbana \& Chicago, University of Illinois Press. 
Engeström, Y. (1999a). "Communication, discourse and activity." The Communication Review 3: 165185.

Engeström, Y. (2000). "Activity theory as a framework for analyzing and redesigning work." Ergonomics 43(7): 960-974.

Engeström, Y. (2001). "Expansive learning at Work: toward an activity theoretical reconceptualization." Journal of Education and Work 14(1): 133-156.

Engeström, Y. (2006). "From Well-Bounded Ethnographies to Intervening in Mycorrhizae Activities." Organization Studies 27(12): 1783-1793.

Engeström, Y. (2007a). From communities of practice to mycorrhizae. Communities of practice: Critical perspectives J. Hughes, N. Jewson and L. Unwin. London, Routledge (pp. 1-20 online at http://www.open.ac.uk/opencetl/resources/details/detail.php?itemld=47690945ef2d9 ).

Engeström, Y., H. Kerosuo, et al. (2007b). "Beyond Discontinuity: Expansive Organizational Learning Remembered." Management Learning 38(3): 319-336.

Etzioni, A. (1961). A comparative analysis of complex organizations. New York, Free Press.

FDI (2008). UAE vital statistics. Foreign Direct Investment http://www.fdimagazine.com/news/fullstory.php/aid/362/UAE: vital statistics.html.

Field, G. C. (1949). The Philosophy of Plato. London, Oxford University Press.

Fischer, G. and J. Ostwald (2001). "Knowledge Management: Problems, Promises, Realities and Challenges." IEEE Intelligent Systems January/February: 60-72.

Floyd, J. (2008). "Towards an Integral renewal of systems methodology for futures studies." Futures 40: 138-149.

Flyvbjerg, B. (2001). Making social sciences matter: why social inquiry fails and how it can succeed again. Cambridge, Cambridge Press.

Flyvbjerg, B. (2006). "Five Misunderstandings About Case-Study Research." Qualitative Inquiry 12(2): 219-245 http://flyvbjerg.plan.aau.dk/Publications2006/0604FIVEMISPUBL2006.pdf.

Folke, C. (2006). "Resilience: The emergence of a perspective for social-ecological systems analyses." Global Environmental Change 16(3): 253-267.

Foucault, M. (1977). Discipline and punish. Harmondsworth, Penguin.

Foucault, M. (1982). The Subject and Power. Michel Foucault: Beyond Structuralism and Hermeneutics. H. L. Dreyfus and P. Rabinow. Chicago, University of Chicago Press: 208 - 228.

Frankenberry, N. (1996). The American Experience. Spirituality and the Secular Quest. P. H. van Ness. New York, The Crossroad Publishing Company: 102-126.

Franklin, S. (1997). "Autonomous agents as embodied Al." Cybernetics and Systems 28(6): 499-520.

Freeman, W. J. (1999). How Brains Make up their Minds. London, Phoenix. 
Freire, P. (1970). Pedagogy of the Oppressed. New York, Seabury.

French, J. and B. H. Raven (1959). The bases of social power. Studies of social power. D. Cartwright. Ann Arbor, MI, Institute of Social Research: 150-167.

Fukuyama, F. (2000). The Great Disruption: Human nature and the reconstitution of social order. New York, Simon \& Schuster.

Gadamer, H. G. (1981). Reason in Age of Science. (Lawrence, F.G. Translation) Cambridge, MA, MIT Press

Gardner, W. L., B. J. Avolio, et al. (2005). "Can you see the real me? A self-based model of authentic leader and follower development." The Leadership Quarterly 16: 343-372.

Geertz, C. (1973). The interpretation of culture. New York, Basic Books.

Gelfand, M. J., Lim, B.C., Raver, J.L. (2004). "Culture and accountability in organizations: Variations in forms of social control across cultures." Human Resource Management Review 14: 135-160.

Gephart, R. P. (1993). "The Textual Approach: Risk and Blame in Disaster Sensemaking." Academy of Management Journal 36: 1465-1514.

Gergen, K. (1985). "Social psychology and the wrong revolution." European Journal of Social Psychology 19: 463-484.

Giddens, A. (1990). Consequences of modernity. Cambridge, Polity Press.

Giddens, A. (1991). Modernity and Self-Identity. Cambridge, Polity Press.

Giddens, A. (2000). Runaway world: How globalization is reshaping our lives. London, BrunnerRoutledge.

Glaser, B. G. and A. L. Strauss (1967). The Discovery of Grounded Theory: Strategies for Qualitative Research. Chicago, Aldine Publishing Company.

Glass, N. (1996). "Chaos, Non-linear Systems and Day-to-day Management." European Management Journal 14(1): 98 - 106.

Glisby, M. and N. Holden (2003). "Contextual constraints in knowledge management theory: the cultural embeddedness of Nonaka's knowledge-creating company." Knowledge and process management 10(1): 29-36.

Green, S. (2006). The management of projects in the construction industry: context, discourse and self-identity. Making Projects Critical. D. Hodgson and S. Cicmil. New York: 232-251.

Grisham, T. and D. Walker (2008). "Cross-cultural leadership." International Journal of Managing Projects in Business 1(3): 439-445.

Guba, E. G. and Y. S. Lincoln (1994). Competing Paradigms in Qualitative Research. Handbook of Qualitative Research. E. G. Guba and Y. S. Lincoln. London, Sage Publications: 105-117.

Gupta, A. K. and V. Govindarajan (2000). "Knowledge flows within multinational corporations." Strategic Management Journal 21: 473-496. 
Habermas, J. (1981). The Theory of Communicative Action, vol 1. Reason and the Rationalization of Society. Boston, Beacon Press.

Habermas, J. (1992). Further reflections on the public sphere. Habermas and the Public Sphere. C. Calhoun. Cambridge, MIT Press: 421-461.

Hall, D. J. and D. Paradice (2005). "Philosophical foundations for a learning-oriented knowledge management system for decision support." Decision Support Systems 39: 445-461.

Hall, W. P. (2003). Organizational Autopoiesis and Knowledge Management. ISD '03 Twelfth International Conference Information System Development. Melbourne, Australia http://www.orgsevolution-

knowledge.net/Index/DocumentKMOrgTheoryPapers/Hall2003OrganizationalAutopoiesisKnowledge Management.pdf.

Hall, W. P. (2005). "Biological nature of knowledge in the learning organisation." The Learning Organisation 12(2): 169-188.

Hamel, G. and C. K. Prahalad (1996). Competing for the Future. USA, Harvard Business School Press.

Hansen, M. T., N. Nohria, et al. (1999). "What's your strategy for managing knowledge?" Harvard Business Review March-April: 106-116.

Hargrave, T. and A. Van de Ven (2006). "A Collective Action Model of Institutional Innovation." Academy of Management Review 31(4): 864-888.

Hasselbladh, H. and J. Kallinikos (2000). "The Project of Rationalization: A Critique and Reappraisal of Neo-Institutionalism in Organization Studies." Organization Studies 21(4): 697-720.

Hazen, M. A. (2001). "Towards Polyphonic Organization." Journal of Organizational Change Management 6(5): 1993.

Healy, S. (2003). "Epistemological pluralism and the politics of choice." Futures 35(7): 689-701.

Heard-Bey, F. (2004). From Trucial States to United Arab Emirates. UAE, Motivate Publishing.

Heidegger, M. (1927). Being and Time. New York, Harper \& Row.

Hejl, P. (1980). The problem of a scientific description of society. Autopoietic Systems in the Social Sciences. F. Benseler, P. Hejl and W. Kock. New York, Penguin.

Held, D. (1991). Political Theory Today. Stanford, Standford University Press.

Held, D., A. McGrew, et al. (1999). "Global Transformations." Retrieved 19 Dec., 2006 from http://www.polity.co.uk/global/.

Hilder, T. (2004). "Viability versus tribalism." Kybernetes 33(3/4): 632-646.

Hobbs, B., M. Aubry, et al. (2008). "The project management office as an organisational innovation." International Journal of Project Management 26(5): 547-555. 
Hodgson, D. and S. Cicmil (2006). Are projects real? The PMBOK and the legitimation of project management knowledge. Making Projects Critical. D. Hodgson and S. Cicmil. New York, Palgrave Macmillan: 29-50.

Hofstede, G. (1980). Culture's consequences: International differences in work-related values. London, Sage.

Hofstede, G. (2001). Culture's Consequences: Comparing values, behaviors, institutions, and organizations across nations. Thousand Oaks, California, Sage Publications.

Hofstede, G. (2003). Culture and organizations: Intercultural cooperation and its importance for survival. Software of the mind. London, Profile Books.

Höijer, B., R. Lidskog, et al. (2006). "Facing dilemmas: Sense-making and decision-making in late modernity." Futures 38: 350-366.

Holling, C. S. (1973). "Resilience and Stability of Ecological Systems." Annual Review of Ecology and Systematics 4: 1-23.

House, R., M. Javidan, et al. (2002). "Understanding cultures and implicit leadership theories across the globe: an introduction to project GLOBE." Journal of World Business 37: 3-10.

House, R. J. and R. N. Aditya (1997). "The Social Scientific Study of Leadership: Quo Vadis?" Journal of Management 23(3): 409-474.

Hughes, B. B., Johnston, P.D. (2005). "Sustainable futures: policies for global development." Futures 37: 813-831.

Husserl, E. (1931). Ideas: General introduction to Pure Phenomenology. (Translated by W.R. Boyce Gibson) London, George Allen \& Unwin.

Iannaccone, L. R. (1998). "Introduction to the economics of religion." Journal of Economic Literature 36(3): 1465-1496.

Il'enkov, E. V. (1977). Dialectical logic: Essays in its history and theory. Moscow, Progress.

Inkpen, A., Beamish, P.W. (1997). "Knowledge, bargaining power and the instability of international joint ventures." Academy of Management Review 22(1): 177-202.

Iskander, S. a. W., K (2004). The readiness to cultivate a knowledge sharing culture as a ground for Knowledge management adoption. Conference on Information Science and Technology 2004, Egypt.

Issawi, C. (1987). (translated and arranged) An Arab Philosophy of History: Selections from the Prolegomena of Ibn Khuldun of Tunis (1332-1406). Princeton, NJ, The Darwin Press Inc.

Ivory, C., N. Alderman, et al. (2006). Sense-making as a process within complex projects. Making Projects Critical. D. Hodgson and S. Cicmil. New York, Palgrave Macmillan: 316-334.

Jaafari, A. (1996). "Human factors in the Australian construction industry: towards total quality management." Australian Journal of Management 21(2): 159-185.

Jackson, M. C. (1991). "The origins and nature of critical systems thinking." Systems Practice 4: 131149. 
James, W. (1975). The Meaning of Truth. Cambridge, Harvard Uni Press.

Järvinen, A. and E. Poikela (2005). The Learning Processes in the Work Organization: From Theory to Design. Learning, Working and Living. Mapping the Terrain of Working Life Learning. E.

Antonacopoulou, P. Jarvis, V. Andersen, B. Elkjaer and S. Høyrup. Basingstoke, Palgrave Macmillan.

Jashapara, A. (2007). "Moving beyond tacit and explicit distinctions: a realist theory of organizational knowledge." Journal of Information Science 33(6): 752-766.

Javidan, M., P. W. Dorfman, et al. (2006). "In the Eye of the Beholder: Cross Cultural Lessons in Leadership from Project GLOBE." Academy of Management Perspectives 20(1): 67-90.

Javidan, M., G. K. Stahl, et al. (2005). "Cross-border transfer of knowledge: Cultural lessons from Project GLOBE." Academy of Management Executive 19(2): 59-76.

Jerez-Gomez, P., J. Cespedes-Lorente, et al. (2005). "Organizational learning capability: a proposal of measurement." Journal of Business Research 58: 715-725.

Jewell, M. and D. Walker (2005). Community of Practice Software Management Tools: A UK Construction Company Case Study. Knowledge Management in the Construction Industry: A SocioTechnical Perspective. Australia, Idea Group.

Johannisson, B. (2008a). Enacting local Economic Development - Theoretical and Methodological Challenges. European Summer University Conference in Entrepreneurship 2008. Bod $\varnothing$ Graduate School of Business and Nordland Research Institute, Bod $\varnothing$ Norway.

Johannisson, B. (2008b) Bengt Johannisson's Prize Lecture: Towards a Practice Theory of Entrepreneuring 2008 Award Winner. International Award for Entrepreneurship and Small Business Research. Växjö University and Jönköping International Business School, Sweden. Volume, http://vxu.se/aktuellt/080925 bengt johannisson/2008Johannison Lecture.pdf 1-12 DOI:

Johannisson, B. and L. Olaison (2007). "The Moment of Truth - Reconstructing Entrepreneurship and Social Capital in the Eye of the Storm." Review of Social Economy LXV(1): 55-78.

Johannisson, B., M. Ramírez, et al. (2002). "The institutional embeddedness of local inter-firm networks: a leverage for business creation." Entrepreneurship \& Regional Development 14: 297-315.

Johnson-Laird, P. N. (1983). Mental Models: Towards a Cognitive Science of Language, Inference and Consciousness. Cambridge, Cambridge University Press.

Kabasakal, H. and M. Bodur (2002). "Arabic cluster: a bridge between East and West." Journal of World Business 37: 40-54.

Kadefors, A. (2004). "Trust in project relationships - inside the black box." International Journal of Project Management 22: 175-182.

Kakabadse, N. K., A. Kouzmin, et al. (2001). "From tacit knowledge to knowledge management: Leveraging invisible assets." Knowledge and process management 8(3): 137-154.

Karwatka, D. (2008). "W.Edwards Deming and Modern Factory Quality Control." Techdirections 67(10): 12.

Katz, D. and R. L. Kahn (1978). The social psychology of organizations. New York, John Wiley. 
Kauffman, S. and S. Levin (1987). "Towards a general theory of adaptive walks on rugged landscapes." Journal of Theoretical Biology 128(1): 11-45.

Kay, R. (2001). "Are Organizations Autopoietic? A Call for New Debate." Systems Research and Behavioural Science 18: 461-477.

Kelly, K. (1998). The New Biology of Business. Rethinking the future. R. Gibson. London, Nicholas Brealey: 250-267.

Kennedy-McColl, J. R. and R. D. Anderson (2005). "Subordinate-manager gender combination and perceived leadership style influences on emotions, self-esteem and organisational commitment." The Journal of Business Research 58: 115-125.

Khaldun, I. (2005). The Muqaddimah: An Introduction to History. Translated Franz Rosenthal (1967). Princeton and Oxford, Princeton University Press.

Kim, D. H. (1993). "The link between individual and organizational learning." Sloan Management Review Fall: 37-50.

Kirat, M. (2005). "Public relations practice in the Arab World: A critical assessment." Public Relations Review 31: 323-332.

Kodama, M. (2005). "New knowledge creation through leadership-based strategic community - a case of new product development in IT and multimedia business fields." Technovation 25: 895-908.

Kodama, M. (2006). "Knowledge-based view of corporate strategy." Technovation 26: 1390-1406.

Kolb, D. A. (1984). Experiential learning: Experience as the source of learning and development. Englewood Cliffs, NJ, Prentice-Hall.

Krause, D. E. (2004). "Influence-based leadership as a determinant of the inclination to innovate and of innovation-related behaviours: An empirical investigation." The Leadership Quarterly 15: 79-102.

Kreiner, K. (1992). "The Postmodern Epoch of Organization Theory." International Studies of Management and Organization 22(2): 37-52.

Kriger, M. and Y. Seng (2005). "Leadership with inner meaning: A contingency theory of leadership based on the worldviews of five religions." The Leadership Quarterly 16: 771-806.

Kuhn, T. (1962). The structure of scientific revolutions. Chicago, Chicago University Press.

Kuran, T. (2004). "The Economic Ascent of the Middle East's Religious Minorities: The Role of Islamic Legal Pluralism." The Journal of Legal Studies 33(2): 475-515.

Ladwig, J. (1994). "For whom this reform? : Outlining educational policy as a social field." British Journal of Sociology of Education 15(3): 341-363.

Lave, J. (1988). Cognition in Practice. Cambridge, Cambridge University Press.

Lave, J. and E. Wenger (1991). Situated Learning: Legitimate peripheral participation. Cambridge, Cambridge University Press.

Law, J. and J. Urry (2004). "Enacting the social." Economy and Society 33(3): 390-410. 
Lawrence, B. (2005). Introduction to the 2005 Edition. The Muqaddimah: An Introduction to History by Ibn Khaldun. N. J. Dawood. Princeton \& Oxford, Princeton University Press: vii - xxv.

Lawrence, T., M. K. Mauws, et al. (2005). "The politics of organizational learning: Integrating power into the $4 \mathrm{I}$ framework." Academy of Management Review 30(1): 180-191.

Lawrence, T. B., M. Winn, et al. (2001). "The temporal dynamics of institutionalization." Academy of Management Review 26: 624-644.

Lee, L. L. (2005). Social capital: The driver for corporate success in the knowledge era. Knowledge management: organisational and Technological Dimensions. J. Davis, Subrahmanian, E., Westerberg, A. New York, Physica-Verlag.

Lee, S. and W.-M. Roth (2003). "Of traversals and hybrid spaces: Science in the community." Mind, Culture, and Activity 10: 120-142.

Leont'ev, A. N. (1981). Problems of the Development of the Mind. Moscow, Progress.

Levin, M. (2003). "Action research and the research community." Concepts and Transformation 8(3): 275-280.

Lewicki, R., D. J. McAllister, et al. (1998). "Trust and distrust: New relationships and realities." The Academy of Management Review 23(3): 438-458.

Lewis, M. and A. Grimes (1999). "Metatriangulation: Building theory from multiple paradigms." Academic Management Review 24(4): 672-690.

Limone, A. and L. E. Bastias (2006). "Autopoiesis and Knowledge in the Organization: Conceptual Foundation for Authentic Knowledge Management." Systems Research and Behavioural Science 23: 39-49.

Lin, C. and S. M. Tseng (2005). "The implementation gaps for the knowledge management system." Industrial Management \& Data Systems 105(2): 208-222.

Lincoln, Y. S. and E. G. Guba (1981). Effective Evaluation. San Francisco, CA, Jossey-Bass.

Lineham, C. and D. Kavanagh (2006). From project ontologies to communities of virtue. Making projects critical. D. Hodgson and S. Cicmil. New York, Palgrave Macmillan: 51-67.

Lingard, B., S. Rawolle, et al. (2005). "Globalizing policy sociology in education: working with Bourdieu." Journal of Education Policy 20(6): 759-777.

Livingston, W. L. (1990). Friends in High Places. New York, FES Publishing Ltd.

Lonergan, B. J. (1972). Method in Theology. London, Longman and Todd.

Loosemore, M. and H. S. Al MusImani (1999). "Construction project management in the Persian Gulf: inter-cultural communication." International Journal of Project Management 17(2, April): 95-100.

Lorenz, E. N. (1963). "Deterministic Nonperiodic Flow." Journal of the Atmospheric Sciences 20(2): 130-141 http://ams.allenpress.com/archive/1520-0469/20/2/pdf/i1520-0469-20-2-130.pdf.

Love, P. E. D., H. Li, et al. (2000). "Total quality management and the learning organization: a dialogue for change in construction." Construction Management and Economics 18: 321-331. 
Luhmann, N. (1995). Social Systems. Standford, CA, Stanford University Press.

Lundin, R. A. and A. Söderholm (1995). "A Theory of the Temporary Organization." Scandinavian Journal of Management 11(4): 437-455.

Lyotard, J. F. (1979). "The Postmodern Condition: A Report on Knowledge." 2007, from http://www.idehist.uu.se/distans/ilmh/pm/lyotard-introd.htm.

Lyotard, J. F. (1984). The postmodern condition: a report on knowledge. Manchester, Manchester University Press.

Lyotard, J. F. (2004). "Introduction to The Postmodern Condition: A Report on Knowledge." 2007, from http://www.idehist.uu.se/distans/ilmh/pm/lyotard-introd.htm.

Macgill, S. M. and Y. L. Siu (2005). "A new paradigm for risk analysis." Futures 37(10): 1105-1131.

Macpherson, A. (2005). "Learning how to grow: resolving the crisis of knowing." Technovation 25: 1129-1140.

Mandelbrot, B. (1983). The Fractal Geometry in Nature. New York, Freeman.

March, J. G. and J. P. Olsen (1975). "The uncertainty of the past: organizational learning under ambiguity." European Journal of Political Research 3: 47-171.

Marsella, A. J. (1998). "Toward a global-community psychology: Meeting the needs of a changing world "American Psychologist 53: 1282-1291.

Marsella, A. J. (2005). "Culture and conflict: Understanding, negotiating, and reconciling conflicting constructions of reality." International Journal of Intercultural Relations 29: 651-673.

Marshall, N. (2006). Understanding power in project settings. Making Projects Critical. D. Hodgson and S. Cicmil. New York, Palgrave Macmillan: 207-231.

Martinsuo, M. (2001). "Balancing large scope and project manageability in a qualitative research design." Management Decision 39(7): 539-550.

Martinsuo, M., N. Hensman, et al. (2006). "Project-based management as an organizational innovation: drivers, changes, and benefits of adopting project-based management." Project Management Journal 37(3): 87-97.

Maturana, H. (1978). Biology of language: The epistemology of reality. Psychology and biology of language and thought: Essays in honor of Eric Lenneberg. G. A. Miller and E. Lenneberg. New York, Academic Press.

Maturana, H. (1980). Biology of cognition. Autopoiesis and Cognition: The Realization of the Living. H. Maturana and F. Varela. Dordrecht, Reidel: 5-58.

Maturana, H. (1988). "Reality: The Search for Objectivity or the Quest for a Compelling Argument." The Irish Journal of Psychology (online access at www.univie.ac.at/constructivism/papers/maturana/88-reality.html) 9(1): 25-82 (online pages 1 32). 
Maturana, H. (1995). "The Nature of Time." Retrieved 2007 online at htttp://www.inteco.cl/biology/nature.htm pp 1-7, .

Maturana, H. (1998). "Human beings versus machines, or machines as instruments of human designs?" Metadesign - Insitituto de Terapia Cognitiva www.inteco.cl/articulos/metadesign.htm.

Maturana, H. (1999). "The Organization of the Living: A theory of the Living Organization." International Journal Human-Computer Studies 51: 149-168.

Maturana, H. (2002). "Autopoiesis, structural coupling and cognition: a history of these and other notions in the biology of cognition." Cybernetics and Human Knowing 9(3-4): 5-34.

Maturana, H. and F. Varela (1980). Autopoiesis and Cognition: The Realization of the Living. Dordrecht, Holland, Reidel.

Maturana, H. and F. Varela (1987). The Tree of Knowledge (first edition). Boston, Shambhala.

Maturana, H. and F. Varela (1998). The Tree of Knowledge: Biological Roots of Human

Understanding. Boston and London, Shambhala.

Maylor, H., T. Brady, et al. (2006). "From projectification to programmification." International Journal of Project Management 24: 663-674.

Mayo, E. (1933). The Human Problems of an Industrial Civilization. New York, Macmillan.

McIntyre-Mills, J. J. (2008). "Reconsidering Relationships Across Self, Others, the Environment and Technology." Systems Research and Behavioral Science 25: 193-213.

Merlau-Ponty, M. (1962). Phenomenology of perception. London, Routledge \& Kegan Paul.

Meyer, J. P., Allen, N.J. (1997). Commitment in the workplace - theory, research, and application. Thousand Oaks, CA, SAGE publications.

Meyers, R. B. (2005). "A Pragmatic Epistemology for Free Choice Learning." Environmental Education Research Feb. 2005(Special edition): 1-8

http://environment.uchicago.edu/studies/pdfs/Meyers 2005 Pragmatic Epistemology.doc.

Michelfelder, D. P. and R. E. Palmer (1989). Introduction. Dialogue and Deconstruction: the GadamerDerrida Encounter. D. P. Michelfelder and R. E. Palmer. Albany, State University of New York Press: 120.

Miles, M. B. and A. M. Huberman (1994). Focusing and Bounding the Collection of Data: Further Design Issues. An Expanded Sourcebook: Qualitative Data Analysis. Thousand Oaks, Sage Publications: 40-43.

Mingers, J. (1990a). "The philosophical implications of Maturana's cognitive theories." Systems Practice 3(6): 569-584.

Mingers, J. (1990b). "The what/how distinction and conceptual models: a reappraisal." Journal of Applied Systems Analysis 17(21-28).

Mingers, J. (2001). "Combining IS Research Methods: Towards a Pluralist Methodology." Information Systems Research 12(3): 240-259. 
Mintzberg, H. (1979). The Structure of Organizations. Englewood Cliffs, NJ, Prentice-Hall.

Modig, N. (2007). "A continuum of organizations formed to carry out projects: Temporary and stationary organization forms." International Journal of Project Management 25: 807-814.

Moghadam, A. H. and P. Assar (2008). "The Relationship Between National Culture and E-Adoption: A Case Study of Iran " American journal of Applie Sciences 5(4): 369-377.

Moingeon, B., and Edmondson, A. (1996). Organizational Learning and Competitive Advantage. London, SAGE Publications.

Montes, J. L., Moreno, A.R., Morales, V.G. (2005). "Influence of support leadership and teamwork cohesion on organizational learning, innovation and performance: an empirical examination." Technovation 25: 1159-1172.

Moores, T. T. and F. H. Gregory (2000). "Cultural Problems in Applying SSM for IS Development " Journal of Global Information Management 8(1): p. 14 e-download pp1-9.

Morgan, P. and S. Taschereau (1996 June). Capacity and institutional assessment: frameworks, methods and tools for analysis. Prepared for CIDA Policy Branch.

Morris, P. (2002). "Science, objective knowledge and the theory of project management." Civil Engineering 150(2): 82-90.

Morrow, R. A. and C. A. Torres (2000). The state, globalization and educational policy. Globalization and Education: Critical Perspectives. N. C. Burbules and C. A. Torres. New York, Routledge: 27-56.

Muczyk, J. P. and D. T. Holt (2008). "Toward a Cultural Contingency Model of Leadership." Journal of Leadership \& Organizational Studies 14(4): 277-286.

Mullins, L. J. (2005). Management and Organisational Behaviour. Britain, Financial Times PrenticeHall.

Munns, A. K. (1995). "Potential influence of trust on the successful completion of a project." International Journal of Project Management 13(1): 19-24.

Nahapiet, J., Ghoshal, S. (1998). "Social capital, intellectual capital, and the organizational advantage." Academy of Management Review 23(2): 242-266.

Naisbitt, J. (1998). From Nation States to Networks. Rethinking the Future. R. Gibson. London, Nicholas Brealey Publishing: 212-226.

Neuman, W. L. (2006). Social Research Methods: Qualitative and Quantitative Approaches. Boston, Pearson Allyn \& Bacon.

Nichols, M. (2002). Development of a quality assurance system for elearning projects. ASCILITE 2002, Auckland, New Zealand http://www.ascilite.org.au/conferences/auckland02/proceedings/papers/004.pdf.

Noland, M. (2005). "Religion and Economic Performance." World Development 33(8): 1215-1232.

Nonaka, I. (1994). "Dynamic theory of organizational knowledge creation." Organizational Scientist 5(1): 14-37. 
Nonaka, I. and H. Takeuchi (1995). The knowledge creating company. New York, Oxford University Press.

Orlikowski, W. J. (2002). "Knowing in Practice: Enacting a Collective Capability in Distributed Organizing." Organization Science 13(3): 249-273.

Orton, J. D. (1996). Reorganizational Learning: Some Conceptual Tools from Weick's Model of Organizing. Organizational Learning and Competitive Advantage B. Moingeon and A. Edmondson. London, Sage Publications: 185-201.

Osborn, R. N., J. G. Hunt, et al. (2002). "Towards a contextual theory of leadership." The Leadership Quarterly 13: 797-837.

Packendorff, J. (1995). "Inquiring into the temporary organization: New directions for project management research." Scandinavian Journal of Management 11(4): 319-333.

Parboteeah, P. and T. W. Jackson (2007). "An Autopoietic Framework for Organisational Learning." Knowledge and Process Management 14(4): 248-259.

Peansupap, V., and Walker, D. (2005). Diffusion of Information and Communication Technology: A Community of Practice Perspective. Knowledge Management in the Construction industry: A Sociotechnical perspective, Idea Group Inc: 89-110.

Peirce, C. S. (1868). "Some Consequences of Four Incapacities." Journal of Speculative Philosophy 2: 140-157 http://www.peirce.org/writings/p27.html.

Perdomo-Ortiz, J., Gonzalez-Benito, J., Galende, J. (2006). "Total quality management as a forerunner of business innovation capability." Technovation 26(10): 1170-1185.

Pettigrew, A. (1987). "Context and action in the transformation of the firm." Journal of Management Studies 24(6): 649-670.

Pettigrew, A. (2001a). "Management Research After Modernism." British Journal of Management 12(Special Issue): S61-S70.

Pettigrew, A., R. W. Woodman, et al. (2001b). "Studying organizational change and development: Challenges for future research." Academy of Management Journal 44(4): 697-713.

Pfeffer, J. (1981). Power in organizations. Marshfield, MA, Pitman.

Phillips, D. and K. Ochs (2004). "Researching policy borrowing: some methodological challenges in comparative education." British Educational Research Journal 30(6): 773-784.

Phillips, N., T. B. Lawrence, et al. (2004). "Discourse and institutions." Academy of Management Review 29(4): 635-652.

Piaget, J. (1937/1954). The construction of reality in the child. New York, NY, Trans M Cook, Basic Books.

Poincaré, H. (1900). "The Theory of Lorentz and The Principle of Reaction." Archives nèerlandaises des Sciences exactes et naturelles 5(2): 252-278 (translated S. Lawrence Jan. 2008 pp.1-27) http://www.physicsinsights.org/poincare-1900.pdf.

Polanyi, M. (1966). The Tacit Dimension. Garden City, NY, Doubleday. 
Pollack, J. (2007). "The changing paradigms of project management." International Journal of Project Management 25: 266-274.

Prahalad, C. K. and G. Hamel (1990). "The core competence of the corporation." Harvard Business Review 68(3): 79-91.

Prentice, W. C. H. (1961). "Understanding Leadership." Harvard Business Review(Jan. 2004): 102-109.

Prigogine, I. (1967). Dissipative structures in chemical systems. Fast Reactions and Primary Processes in Chemical Kinetics. S. Claessons. New York, Interscience.

Proulx, J. (2008). "Some Differences between Maturana and Varela's Theory of Cognition and Constructivism." Complicity: An International Journal of Complexity and Education 5(1): 11-26.

Pundir, A., L. Ganapathy, et al. (2007). "Towards a complexity framework for managing projects." Emergence: Complexity and Organization 9(4): 17-25.

Raelin, J. A. (2000). Work-Based Learning. Upper Saddle, NJ, Prentice-Hall.

Rahman, S. (2007). Strong emphasis on social sector. Gulf News. UAE, Gulfnews.com http://archive.gulfnews.com/articles/.

Reason, P. and W. R. Torbert (2001). "The action turn: toward a transformational social science." Concepts and Transformation 6(1): 1-38.

Reid, D. A. (1996). Enactivism as a methodology. Twentieth Annual Conference of the International Group of Mathematics Education, Valencia, Spain.

Reisman, A. and M. Oral (2005). "Soft Systems Methodology: A Context Within a 50-Year Retrospective of OR/MS." Interfaces 35(2): 164-178.

Renzl, B. (2008). "Trust in management and knowledge sharing: The mediating effects of fear and knowledge documentation." Omega 36: 206-220.

Richardson, K. (2008). "Managing complex organizations: complexity thinking and the science and art of management." Emergence: Complexity and Organization 10(2): 13 (Online version pp.1-18).

Richey, R. and J. D. Klein (2007). Design and Development Research: Methods Strategies and Issues. NJ, USA, Lawrence Erlbaum Associates Inc.

Riegler, A. (2002). "When is a cognitive system embodied?" Cognitive Systems Research 3: 339-348.

Robbins, D. (2000). Bourdieu \& Culture. London, Sage Publications.

Robinson, V. M. J. (2001). "Descriptive and normative research on organisational learning: Locating the contribution of Argyris and Schon." The International Journal of Educational Management 15(2): 58-67.

Rogers, E. M. (1995). Diffusion of innovation. New York, The Free Press.

Rosenau, P. M. (1991). Post-modernism and the social sciences: Insights, inroads, and intrusions. New Jersey, Princeton University Press. 
Rowley, J. (2003). "Action research: an approach to student work based learning." Education and Training 45(3): 131-138.

Rust, V. D. (2000). Education policy studies and comparative education. Comparative Study of Educational Policy. R. Alexander, M. Osborn and D. Phillips. Oxford, Symposium Books: 13-39.

Ryle, G. (1949). The Concept of the Mind. London, Penguin Books.

Sachs, J. (1994). "Strange yet compatible bedfellows: Quality assurance and quality improvement." Australian Universities Review 37(1): 22-25.

Saha, L. (2000). The sociology of comparative education. Sociology of Education. J. Demaine. Basingstoke, Palgrave: 163-179.

Sankaran, S., T. B. Hou, et al. (2009). "Managing organizational change by using soft systems thinking in action research projects." International Journal of Managing Projects in Business 2(2).

Sankaran, S. and B. H. Tay (2007) Are Interpretative and Critical Research Methods Useful for Research in Project Management. AIPM 2007 Volume, 1-13

http://www.aipm.com.au/resource/AIPM2007 Sankaran InterpretativeandCritical-ResearchMethods-CS313.pdf DOI:

Scharmer, C. O. (2001). Self-transcending Knowledge: Organizing Around Emerging Realities. Managing Industrial Knowledge - creation, transfer and utilization. London, Sage: 68-90.

Scharmer, C. O. and F. Varela (2000) The Three Gestures of Becoming Aware: Conversation with Francisco Varela. http://www.dialogonleadership.org/varela-2000.html

Volume, DOI:

Scheepers, R., K. Venkitachalam, et al. (2004). "Knowledge strategy in organizations: refining the model of Hansen, Nohria and Tierney." Journal of Strategic Information Systems 13: 201-222.

Schegloff, E. (1992). "Repair after the next turn: The last structurally provided defense of intersubjectivity in conversation." American Journal of Sociology 97: 1295 - 1345.

Schutz, A. (1964). The stranger: an essay in social psychology. Collected Papers Vol II Studies in Social Theory. The Hague: Martinus Nijhoff.

Schutz, A. (1967). The Phenomenology of the Social World. Evanston, III, Northwestern University Press.

Schwaninger, M. (2006). "System Dynamics and the Evolution of the Systems Movement." Systems Research and Behavioral Science(23).

Scott, A. (2003) Husserl's Ideas on a Pure Phenomenology and on a Phenomenological Philosophy. http://www.angelfire.com/md2/timewarp/husserl.htm. Volume, DOI:

Searle, J. (1999). Mind, Language and Society. London, Phoenix.

SEI. (2009). "Capability Maturity Model - Integrated." Retrieved 20th January, 2008, from http://www.sei.cmu.edu/cmmi/general/index.html.

Senge, P. (1990). The Fifth Discipline. London, Random House. 
Sense, A. (2003). "A model of the politics of project leader learning." International Journal of Project Management 21: 107-114.

Shamir, B. and J. M. Howell (1999). "Organizational and contextual influences on the emergence and effectiveness of charismatic leadership." Leadership Quarterly 10(2): 257-283.

Shenhar, A. J. (1998). "From Theory to Practice: Toward a Typology of Project Management Styles." IEEE Transactions on Engineering Management 45(1): 33-48.

Shewart, W. A. (1931). Economic Control of Quality of Manufactured Product. New York, D, Van Nostrand.

Sikka, T. (2008). "Pragmatics, poststructuralism, and hermeneutics: An examination of discursiveconsensus formation and its ethical implications." Journal of Pragmatics 40(2): 227-243.

Simco, N. and J. Warin (1997). "Validity in Image-based Research: an elaborated illustration of the issues." British Educational Research Journal 23(5): 661-672.

Simon, H. A. (1945). Administrative Behavior. New York, Macmillan.

Skinner, B. F. (1974). About behavioursm. London, Cape Press.

Small, J. (2006). Leaders, Learners and Laggards: Capacity planning for organisational effectiveness. Quality Assurance in Higher Education: Mission (Im)possible?, Pretoria, South Africa http://www.fotim.ac.za/fotim/fotim conferences/qaconference2006/papers/paper small.pdf.

Smith, L. B. (2005). "Cognition as a dynamic system: Principles from embodiment." Developmental Review 25: 278-298.

Söderlund, J. (2004a). "Building theories of project management: past research, questions for the future." International Journal of Project Management 22(183-191).

Söderlund, J. (2004b). "On the broadening scope of the research on projects: a review and a model for analysis." International Journal of Project Management 22: 655-667.

Sommer, S. C. and C. H. Loch (2004). "Selectionism and Learning in Projects with Complexity and Unforeseeable Uncertainty." Management Science 50(10): 1334-1347.

Spender, J. C. (1996). Competitive Advantage from Tacit Knowledge? Unpacking the Concept and its Strategic Implications. Organizational Learning and Competitive Advantage. B. Moingeon, and Edmondson, A. London, Sage publications: 56-73.

Spender, J. C. (1996b). "Making knowledge: The basis of a dynamic theory of the firm." Strategic Management Journal 17 (Special Issue): 45-62.

Stackman, R. W., L. S. Henderson, et al. (2006). "Emergence and community: The story of three complex adaptive entities." Emergence: Complexity and Organization 8(3): 78-91.

Stewart, D. and D. Waddell (2008). "Knowledge Management: The fundamental component for delivery of quality." Total Quality Management 19(9): 987-996.

Sveiby, K. E. (2001). "A knowledge-based theory of the firm to guide in strategy formulation." Journal of Intellectual Capital 2(4): 344-358. 
Szulanski, G. (1996). "Exploring internal stickiness: Impediments to the transfer of best practice within the fiRM." Strategie Management Journal 17(Winter Special issue): 27-43.

Szulanski, G. (2003). Sticky knowledge barriers to knowing in the firm. Thousand Oaks, CA, Sage Publications.

Szulanski, G. and R. J. Jensen (2004). "Overcoming stickiness: An empirical investigation of the role of the template in the replication of organizational routines." Managerial and Decision Economics 25: 347-363.

Taylor, F. W. (1911). The Principles of Scientific Management. New York, Harper and Brothers.

Teece, D. J. (1998). "Capturing Value from Knowledge Assets: The New Economy, Markets for KnowHow and Intangible Assets." Californian Management Review 40(3): 55-79.

Thomas, J. B., S. M. Clark, et al. (1993). "Strategic sensemaking and organizational performance: Linkages among scanning, interpretation, action and outcomes." Academy of Management journal 36(2): 239-270.

Thomas, J. L. and J. Tjader (2000). On learning and control - competing paradigms or co-existing requirments for managing projects in ambiguous situations? Fourth biannual conference of the international research network on managing by projects, Sydney, Australia.

Thomson, J. A. K. (1955). The Ethics of Aristotle. (translation) Harmondsworth, Middlesex, Penguin Books.

Townley, B. (2002). "The role of competing rationalities in institutional change." Academy of Management Journal 45(1): 163-179.

Tsoukas, H. (1996). "The firm as a distributed knowledge system: A constructionist approach." Strategic Management Journal 17: 11-25.

Tsoukas, H. and R. Chia (2002). "On Organisational Becoming: Rethinking Organisational Change." Organization Science 13(5): 567-582.

Turner, J. R. (1999). The Handbook of Project-Based Management. London, McGraw-Hill Company.

Turner, J. R. (2006a). "Towards a theory of project management: The nature of the project (Editorial)." International Journal of Project Management 24: 1 - 3.

Turner, J. R. (2006b). "Towards a theory of Project Management: The Functions of Project Management (Editorial)." International Journal of Project Management 24: 187 - 189.

Turner, J. R. (2006c). "Towards a theory of project management: The nature of the functions of project management (Editorial)." International Journal of Project Management 24: 277 - 279.

Turner, J. R. and R. A. Cochrane (1993). "Goals-and-methods matrix: coping with projects with ill defined goals and/or methods of achieving them." International Journal of Project Management 11(2): $93-102$.

Turner, J. R. and R. Müller (2003). "On the nature of the project as a temporary organization." International Journal of Project Management 21: 1-8. 
Tyler, L. (2005). "Towards a postmodern understanding of crisis communication." Public Relations Review 31: 566-571.

UAE Ministry of Planning, U. A. E. (2003). "EIU World Prospects." CIA Year Book http://www.fdimagazine.com/news/fullstory.php/aid/362/UAE: vital statistics.html.

Ueda, Y., J. S. Thomsen, et al. (1993). "Behaviour of the Solution to Duffing's Equation for Large Forcing Amplitudes." Mathematical Research 72: 149-166.

Van de Ven, A. (1986). "Central problems in the management of innovation." Management Science 32(5): 590-607.

Van de Ven, A. (2005). "Running in Packs to Develop Knowledge-Intensive Technologies." MIS Quarterly 29(2): 365-378.

van Donk, D. P. and E. Molloy (2008). "From organising as projects to projects as organisations." International Journal of Project Management 26: 129-137.

van Eijnatten, F. M. (2004). "Chaordic systems thinking: Some suggestions for a complexity framework to inform a learning organization." The Learning Organization 11(6): 430-449.

Varela, F. (1979). Principles of biological autonomy. New York, North Holland.

Varela, F. (1987). Laying down a path in walking. Gaia: A way of knowing. W. Thompson. Hudson, NY, Lindisfarne Press: 65-82.

Varela, F. (2000). Why the proper study of mind implies the transcendence of nature. Religion in Mind: Cognitive Science and Religious Experience. J. Andresen. UK, Cambridge University Presss.

Varela, F., H. Maturana, et al. (1974). "Autopoiesis: The organization of living systems, its characterization and a model." BioSystems 5: 187-196.

Varela, F., E. Thompson, et al. (1991). The Embodied Mind: Cognitive science and human experience. Cambridge, Mass., MIT Press.

Vaughan, G. M. and M. A. Hogg (2005). Introduction to Social Psychology. Australia, Pearson, Prentice Hall.

Veitch, J. (2002). (translated) Selections from the Principles of Philosophy of René Descartes (15961650): Part I - Of the Principles of Human Knowledge, The Classical Library.

http://www.classicallibrary.org/descartes/principles/01.htm.

Vickers, G. (1965). The Art of Judgement. London, Chapman and Hall (Reprinted 1983 Harper and Row, London).

von Bertalanaffy, L. (1968). General System Theory: Foundations, Development, Applications. New York, George Braziller.

von Glasersfeld, E. (1979). Cybernetics, Experience and the Concept of Self. A cybernetic approach to assessment of children: Toward a more humane use of human beings. M. N. Ozer. Boulder, CO, Westview Press: 67-113. 
von Glasersfeld, E. (1990). An Exposition of Constructivism: Why Some Like it Radical. Monographs of the Journal for Research in Mathematics Education. R. B. Davis, Maher, C.A., Noddings, N. Reston, VA, National Council of Teachers of Mathematics. 4: 19-29.

von Hayek, F. A. (1974). The Pretence of Knowledge. Nobel Lectures, Economics 1969-1980. A. Lindbeck, World Scientific Publishing Co., Singapore, 1992.

von Krogh, G., K. Ichijo, et al. (2000). Enabling Knowledge Creation: How to Unlock the Mystery of Tacit Knowledge nad release the Power of Innovation. New York, Oxford University Press.

Vroeijenstijn, T. (1990). "Autonomy and Assurance of Quality: Two Sides of the One coin: The case of quality assessment in Dutch universities." Higher Education Research and Development 9(1): 21-38.

Vygotsky, L. S. (1978). Mind in society: the development of higher psychological functions.

Cambridge, MA, Harvard University Press.

Vygotsky, L. S. (1986). Thought and Language. Cambridge, MA, MIT Press.

Waddell, D., Sohal, A. (1998). "Resistance: a constructive tool for change management." Management Decision 36(8): 543-548.

Waldrop, M. (1992). Complexity: The emerging science at the edge of order and chaos. New York, Touchstone.

Walker, D. (2004). The Knowledge Advantage (K-Adv): Unleashing Creativity and innovation. Melbourne, Australia, RMIT University.

Walker, D., L. Bourne, et al. (2008). "Influence, stakeholder mapping and visualization." Construction Management and Economics 26(6): 645-658.

Walker, D. and D. Christenson (2005). "Knowledge wisdom and networks: a project management centre of excellence example." The Learning Organization 12(3): 275-291.

Walker, D., T. Maqsood, et al. (2005). The Culture of the Knowledge Advantage (K-Adv) - An Holistic Strategic Approach to the Management of Knowledge. Knowledge Management in the Construction Industry: A Socio-Technical Perspective. A. S. Kazi. Hershey, PA, Idea Group: 223-248.

Walker, D., A. J. Wilson, et al. (2004). The Knowledge Advantage (K-Adv) For Unleashing creativity \& Innovation in the Construction Industry Melbourne, RMIT: 1-32.

Watling, R. (1995). Practical media work in education: practices, problems and strategies. British Educational Research Association day conference. Lancaster university.

Watson, K. (2007). "Language, education and ethnicity: Whose rights will prevail in an age of globalisation?" International Journal of Educational Development Article in Press:

doi:10.1016/j.ijedudev.2006.10.015: 1-13.

Watson, W. E., K. Kumar, et al. (1993). "Cultural diversity's impact on interaction process and performance: Comparing homogenous and diverse task groups." The Academy of Management Journal 36(3): 590-602.

Weber, M. (1978). Economy and Society. (Eds. G.Roth and C. Wittich) Berkley, University of California Press. 
Weick, K. E. (2001). Making sense of the organization. United Kingdom, Blackwell Publishing.

Weick, M. (1978). Economy and Society. Thousand Oaks, CA, Sage.

Wenger, E. (1999). Communities of Practice: Learning, Meaning, and Identity. USA, Cambridge University Press.

Wernerfelt, B. (1984). "A Resource-based View of the Firm." Strategic Management Journal 5: 171180.

Whitaker, R. (1995) Self-organization, autopoiesis and enterprises. http://bat710.univIyon1.fr/ imathon/autopoesis/Main.html Volume, DOI:

Whittington, R., A. Pettigrew, et al. (2001). Conclusion: Doing More in Strategy Research. $\underline{\text { Handbook }}$ of Strategy and Management. A. Pettigrew, H. Thomas and R. Whittington. London, Sage: 447-490.

Whitty, S. J. (2005). "A memetic paradigm of project management." International Journal of Project Management 23: 575-583.

Whitty, S. J. and H. Maylor (2007). And then came Complex Project Management. 21st IPMA World Congress on Project Management, Cracow, Poland.

Whitty, S. J. and M. F. Schulz (2007). "The impact of Puritan ideology on aspects of project management." International Journal of Project Management 25: 10-20.

Williams, T. (1999). "The need for new paradigms for complex projects." International Journal of Project Management 17(5): 269-273.

Williams, T. (2002). Modelling Complex Projects. London, Wiley.

Wilson, T. D. (2002). Alfred Schutz, phenomenology and research methodology for information behaviour research. ISIC4 - Fourth International Conference on Information Seeking in Context, Universidade Lusiada, Lisbon, Portugal. http://informationr.net/tdw/publ/papers/schutz02.html.

Windschuttle, K. (2002). The Ethnocentrism of Clifford Geertz, Foundation for Cultural Review, http://science.jrank.org/pages/9236/Ethnocentrism-Conclusion.html

Winter, M. (2006). "Problem structuring in project management: an application of soft systems methodology (SSM)." Journal of the Operational Research Society 57: 802-812.

Winter, M. and P. Checkland (2003). "Soft systems: a fresh perspective for project management." Civil Engineering 156(November): 187-192.

Winter, M., C. Smith, et al. (2006). "Directions for future research in project management: the main findings of a UK government-funded research network." International Journal of Project Management 24: 638-649.

Winter, S. G. (1987). Knowledge and competence as strategic assets. The Competitive Challenge. D. J. Teece. Cambridge, MA, Ballinger: 159-184.

Wittgenstein, L. (1953). Philosophical Investigations. Oxford, Blackwell.

Wolin, S. S. (1988). On the theory and practice of power. After Foucalt. J. Arac. New Brunswick and London, Rutgers University Press: 179-201. 
Yamazaki, Y. (2005). "Learning styles and typologies of cultural differences: A theoretical and empirical comparison." International Journal of Intercultural Relations 29(5): 521-548.

Yin, R. K. (2003). Case study research: Design and methods. (3rd edition) Newbury Park, CA, Sage.

Yu, J. E., H. K. Moon, et al. (2008). "Proposing Rhizomatic Systems Methodology: Extending

Churchman's pragmatism to Post-Structuralism." Systems Research and Behavioral Science 25: 291303.

Yukl, G. (2002). Leadership in Organizations. New Jersey, Prentice Hall.

Yurtseven, M. K. (1999). "Systems Engineering and Soft Systems Methodology: A Review." Dogus University Journal 1: 225-230.

Zack, M. (1999). "Developing a Knowledge Strategy." Californian Management Review 41(3): 125145.

Zagorsek, H., M. Jaklic, et al. (2004). "Comparing leadership practices between the United States, Nigeria and Slovenia: Does culture matter?" Cross Cultural management 11(2): 16-34.

Zeleny, M. (1977). "Self-organization of living systems: a formal model of autopoiesis." International Journal of General Systems 4(13-28).

Zollo, M. and S. Winter (2002). "Deliberate learning and the evolution of dynamic capabilities." Organization Science 13(3): 339-351. 


\section{Appendix 1}

\section{College PQA Recommendations - Summary}

\section{3-2004}

- The College should clarify more clearly the relationship for itself between divisional and college quality work including the responsibility for data gathering and dissemination

- The College should undertake a fundamental review of the college quality processes with a view to identifying those which are generating data for quality purposes and those which are administrative procedures.

- There are a number of processes which do not lend themselves to effective evidence gathering and analysis

- There needs to be more effective means of data storage and documentation of evidence.

- In future cycles of PQA the use of processes for data analysis needs to be extended for decision making purposes

- In future cycles, data gathered from processes in place needs to be used for broader analysis which could be used for program enhancement.

- There needs to be further clarity in evidence for KC4, with the College identifying barriers to student assessment and progression within programs

\section{4-2005}

- The College needs to enhance the data analysis, interpretation and action planning phases of their quality assurance system

- There is a need for focusing and fine tuning of the quality assurance processes

- A review of the processes is needed as some are more administrative than quality assurance

- A number of new processes have not generated data as yet, thus this will need to be monitored in the next quality cycle

- The College needs to consider how data being generated can be analyzed and interpreted to address program level questions

- Formal processes need to be established to action recommendations and issues from previous years

- Process for review for effectiveness of QA processes needs to be strengthened 
Applied Business Diploma \& Financial Services \& Banking (2004_05):

- Tools for data collection processes need identifying

- Insufficient data analysis and interpretations for a number of processes at the program level

- Conclusions need to link with quality assurance data and interpretation

- Action planning needs to be linked more strongly with data analysis and interpretation

- Formal processes need to be established to action recommendations and issues from previous years 


\section{Appendix 2}

\section{College QA Planning Group Feedback (26/10/05)}

1. Common tools and processes

2. Sub-taskforce to review:
a. Common processes for naming conventions
b. Process content
c. Service area KCs in alignment with Common (program KCs)

3. Meeting - Tuesday $15^{\text {th }}$ November to resolve the above and make recommendations for full QA planning group meeting $28^{\text {th }}$ November. Meeting will be held at XX College to view XX portal and QA applications.

4. Review of GOs for $2005-2006$

5. Recommendation to review GO7 as per last year, not 1-6. That is $\mathrm{GO7}$ will be reviewed via DAT administered KCA, with programs in Colleges needing to provide the evidence of administration and moderation.

6. QA for Service Areas - will proceed, however, confirmation of KCs or recommendation to Policy Committee, will take place after next full meeting $28^{\text {th }}$ October.

7. Suggested action: for Colleges in the interim, consider Mission \& Objectives of Service areas and refer to Common (Program) KCs for guidance in process establishment. Set up shared drive data storage mechanism for data collection, evidence etc.

8. No specific timelines have been set at this stage.

9. Program Mission and objective statements, should be being developed at the DAT level. These will need to be included in web pages.

10. Programs for review 2005-06: Engineering (HDs \& Bachelors); Business (HDs \& Bachelors), Information Technology (Diploma, HD \& Bachelors).

11. Program offerings lists been confirmed by XX \& XX.

12. No XX program to be reviewed this year.

13. Faculty profile updates view for data entry - ready to go tomorrow. XX please let your faculty know, and impress upon them importance. Note: this should be an update, all other info from last year should be in there. Problems with data entry - to be discussed with IT support. 
14. QA Planning group to develop Terms of Reference.

15. Checklist for audit purposes to follow template format, which is still be finalized.

16. Portal integration - awaiting Policy Committee decision, however, appears XX portal will be the model used. Awaiting confirmation from XX re interface between ILE new data base and XX portal. 


\section{Appendix 3}

\section{College QA 2005-2006 timeline}

\begin{tabular}{|c|c|c|c|c|c|c|c|c|c|}
\hline Task & Description & Oct '05 & Nov '05 & Dec '05 & Jan '06 & Feb '06 & March '06 April '06 & May '06 & June '06 \\
\hline 1 & College Management & & & & & & & & \\
\hline $1.1 \mathrm{r}$ & Revision Common HCT QA processes & & & & & & & & \\
\hline 1.2 & Confirm College QA processes & & & & & & & & \\
\hline 1.3 & Revise process content, identify tools/responsibilities & & & & & & & & \\
\hline 1.4 & CQAB formation \& meetings & & & & & & & & \\
\hline & & & & & & & & & \\
\hline 2 & Academic Programs & & & & & & & & \\
\hline 2.1 & Revise Program processes in alignment with College & & & & & & & & \\
\hline 2.21 & Identify faculty responsible for process implementation & & & & & & & & \\
\hline 2.4 & Data entry into data base & & & & & & & & \\
\hline 2.5 & Develop Departmental web pages & & & & & & & & \\
\hline & & & & & & & & & \\
\hline 3 & Support Services & & & & & & & & \\
\hline 3.1 & Revision Common HCT QA processes & & & & & & & & \\
\hline 3.2 & Confirm College SS QA processes & & & & & & & & \\
\hline 3.3 & Revise SService processes in alignment with College & & & & & & & & \\
\hline 3.4 & Revise process content, identify tools/responsibilities & & & & & & & & \\
\hline 3.5 & Data entry into data base & & & & & & & & \\
\hline 3.6 & Develop Departmental web pages & & & & & & & & \\
\hline & & & & & & & & & \\
\hline 4 & Data management & & & & & & & & \\
\hline 4.11 & ADW PQA Web page design & & & & & & & & \\
\hline 4.2 & Develop interface b/w QA web page \& QA data base & & & & & & & & \\
\hline 4.3 & Develop interface b/w Webpage \& Depart. pages & & & & & & & & \\
\hline 4.45 & Support data entry into data base & & & & & & & & \\
\hline 4.5 & Support Develop Departmental web pages & & & & & & & & \\
\hline & & & & & & & & & \\
\hline 5 & Reporting process & & & & & & & & \\
\hline 5.1 & Report submission to ILE & & & & & & & & \\
\hline 5.2 & Feedback questions & & & & & & & & \\
\hline 5.3 & Review visit & & & & & & & & \\
\hline
\end{tabular}




\section{Appendix 4}

\section{College QA Data Checklist ‘05-`06 Academic Chairs}

\begin{tabular}{|c|c|c|c|c|c|c|c|c|}
\hline \multirow[b]{2}{*}{ Key Criteria } & \multicolumn{3}{|c|}{ College responsibilities } & \multicolumn{5}{|c|}{ Program responsibilities } \\
\hline & & Process & Data Gathering & $\begin{array}{l}\text { Data } \\
\text { Analysis }\end{array}$ & Data Interpretation & Action Planning & $\begin{array}{l}\text { Review of } \\
\text { Action Plan }\end{array}$ & Process/Review \\
\hline $\begin{array}{l}\text { Primary reporting } \\
\text { group } \\
\text { (Link to) }\end{array}$ & $\begin{array}{l}\text { Does the college } \\
\text { have appropriate } \\
\text { tools to collect } \\
\text { data against this } \\
\text { Key Criterion? }\end{array}$ & $\begin{array}{l}\text { Does the college have a } \\
\text { clear administrative } \\
\text { process governing the } \\
\text { use of these tools? }\end{array}$ & $\begin{array}{l}\text { Has the college } \\
\text { followed its } \\
\text { admin processes } \\
\text { in the collection } \\
\text { of data in this } \\
\text { current QA cycle? }\end{array}$ & $\begin{array}{l}\text { Has the } \\
\text { collected } \\
\text { data been } \\
\text { analyzed by } \\
\text { program? }\end{array}$ & $\begin{array}{l}\text { Has the analysis been } \\
\text { interpreted and } \\
\text { conclusions drawn } \\
\text { resulting in areas for } \\
\text { action being identified } \\
\text { and/or changes made? }\end{array}$ & $\begin{array}{l}\text { For each } \\
\text { identified area } \\
\text { for action, has a } \\
\text { plan been } \\
\text { developed to } \\
\text { address the } \\
\text { issues? }\end{array}$ & $\begin{array}{l}\text { Does a process } \\
\text { exist for the } \\
\text { review of } \\
\text { progress against } \\
\text { identified } \\
\text { actions in } \\
\text { previous plans? }\end{array}$ & $\begin{array}{l}\text { Has a review of the } \\
\text { effectiveness of the } \\
\text { process/tool used been } \\
\text { carried out? }\end{array}$ \\
\hline $\begin{array}{l}\text { KC1 } \\
\text { Stakeholder } \\
\text { satisfaction }\end{array}$ & & 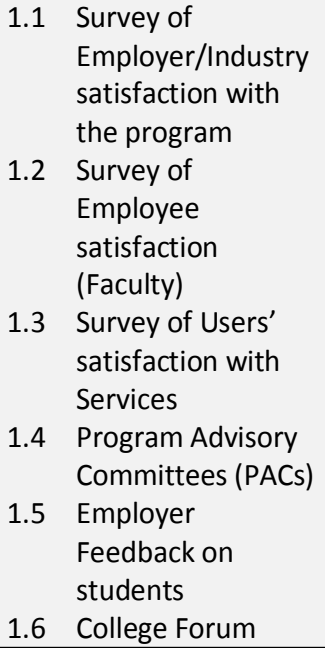 & & & & & & \\
\hline $\begin{array}{l}\text { KC2 } \\
\text { Professional and } \\
\text { external } \\
\text { benchmarking and } \\
\text { recognition (DAT) }\end{array}$ & & $\begin{array}{l}\text { 2.1 External Evaluation } \\
\text { of Non-Accredited } \\
\text { Programs } \\
\text { 2.2 Review of Program } \\
\text { Against Recognized } \\
\text { Standards }\end{array}$ & & & & & & \\
\hline
\end{tabular}




\begin{tabular}{|c|c|c|c|c|c|c|c|c|}
\hline & \multicolumn{3}{|c|}{ College responsibilities } & \multicolumn{5}{|c|}{ Program responsibilities } \\
\hline & Tools & Process & Data Gathering & Data Analysis & $\begin{array}{l}\text { Data } \\
\text { Interpretation }\end{array}$ & Action Planning & $\begin{array}{l}\text { Review of } \\
\text { Action Plan }\end{array}$ & Process/Review \\
\hline $\begin{array}{l}\text { Primary reporting group } \\
\text { (Link to) }\end{array}$ & $\begin{array}{l}\text { Does the } \\
\text { college } \\
\text { have } \\
\text { appropriate } \\
\text { tools to } \\
\text { collect data } \\
\text { against this } \\
\text { Key } \\
\text { Criterion? }\end{array}$ & $\begin{array}{l}\text { Does the college have a clear } \\
\text { administrative process } \\
\text { governing the use of these } \\
\text { tools? }\end{array}$ & $\begin{array}{l}\text { Has the college } \\
\text { followed its } \\
\text { admin } \\
\text { processes in the } \\
\text { collection of } \\
\text { data in this } \\
\text { current QA } \\
\text { cycle? }\end{array}$ & $\begin{array}{l}\text { Has the } \\
\text { collected data } \\
\text { been } \\
\text { analyzed by } \\
\text { program? }\end{array}$ & $\begin{array}{l}\text { Has the analysis } \\
\text { been interpreted } \\
\text { and conclusions } \\
\text { drawn resulting in } \\
\text { areas for action } \\
\text { being identified } \\
\text { and/or changes } \\
\text { made? }\end{array}$ & $\begin{array}{l}\text { For each identified } \\
\text { area for action, } \\
\text { has a plan been } \\
\text { developed to } \\
\text { address the } \\
\text { issues? }\end{array}$ & $\begin{array}{l}\text { Does a process } \\
\text { exist for the } \\
\text { review of } \\
\text { progress against } \\
\text { identified } \\
\text { actions in } \\
\text { previous plans? }\end{array}$ & $\begin{array}{l}\text { Has a review of the } \\
\text { effectiveness of the } \\
\text { process/tool used been } \\
\text { carried out? }\end{array}$ \\
\hline $\begin{array}{l}\text { KC3 } \\
\text { Alignment of Academic } \\
\text { Programs and Support } \\
\text { Service Delivery with } \\
\text { HCT Graduate Outcomes } \\
\text { (DAT) }\end{array}$ & & $\begin{array}{l}\text { 3.1 Aligning Course Goals with } \\
\text { Graduate Outcomes } \\
\text { 3.2 Course Outline Review } \\
\text { 3.3 Course Review } \\
\text { 3.4 Key Common Assessment } \\
\text { Review } \\
\text { 3.5 Course Sequencing Review } \\
\text { 3.6 Audit of Course } \\
\text { Assessment Files } \\
\text { 3.7 Program Rationalization } \\
\text { and Design }\end{array}$ & & & & & & \\
\hline $\begin{array}{l}\text { KC4 } \\
\text { Student performance in } \\
\text { assessment and } \\
\text { progression through the } \\
\text { program. }\end{array}$ & & $\begin{array}{l}\text { 4.1 Analysis of student grades } \\
\text { to: } \\
\text { i) determine obstacles to } \\
\text { student success } \\
\text { ii) determine the grades to } \\
\text { determine the } \\
\text { appropriateness of program } \\
\text { difficulty levels } \\
4.2 \text { Cohort tracking }\end{array}$ & & & & & & \\
\hline
\end{tabular}




\begin{tabular}{|c|c|c|c|c|c|c|c|c|}
\hline & \multicolumn{3}{|c|}{ College responsibilities } & \multicolumn{5}{|c|}{ Program responsibilities } \\
\hline & Tools & Process & Data Gathering & Data Analysis & $\begin{array}{l}\text { Data } \\
\text { Interpretation }\end{array}$ & Action Planning & $\begin{array}{l}\text { Review of } \\
\text { Action Plan }\end{array}$ & Process/Review \\
\hline $\begin{array}{l}\text { Primary reporting group } \\
\text { (Link to) }\end{array}$ & $\begin{array}{l}\text { Does the } \\
\text { college } \\
\text { have } \\
\text { appropriate } \\
\text { tools to } \\
\text { collect data } \\
\text { against this } \\
\text { Key } \\
\text { Criterion? }\end{array}$ & $\begin{array}{l}\text { Does the college have a clear } \\
\text { administrative process } \\
\text { governing the use of these } \\
\text { tools? }\end{array}$ & $\begin{array}{l}\text { Has the college } \\
\text { followed its } \\
\text { admin } \\
\text { processes in the } \\
\text { collection of } \\
\text { data in this } \\
\text { current QA } \\
\text { cycle? }\end{array}$ & $\begin{array}{l}\text { Has the } \\
\text { collected data } \\
\text { been } \\
\text { analyzed by } \\
\text { program? }\end{array}$ & $\begin{array}{l}\text { Has the analysis } \\
\text { been interpreted } \\
\text { and conclusions } \\
\text { drawn resulting in } \\
\text { areas for action } \\
\text { being identified } \\
\text { and/or changes } \\
\text { made? }\end{array}$ & $\begin{array}{l}\text { For each identified } \\
\text { area for action, } \\
\text { has a plan been } \\
\text { developed to } \\
\text { address the } \\
\text { issues? }\end{array}$ & $\begin{array}{l}\text { Does a process } \\
\text { exist for the } \\
\text { review of } \\
\text { progress against } \\
\text { identified } \\
\text { actions in } \\
\text { previous plans? }\end{array}$ & $\begin{array}{l}\text { Has a review of the } \\
\text { effectiveness of the } \\
\text { process/tool used been } \\
\text { carried out? }\end{array}$ \\
\hline $\begin{array}{l}\text { KC5 } \\
\text { Student and graduate } \\
\text { satisfaction with } \\
\text { teaching, learning and } \\
\text { assessment. }\end{array}$ & & $\begin{array}{ll}5.1 & \begin{array}{l}\text { Student feedback on } \\
\text { faculty teaching and } \\
\text { learning }\end{array} \\
5.2 & \begin{array}{l}\text { Student feedback on } \\
\text { courses and materials }\end{array} \\
5.3 & \text { Exit survey - Graduates } \\
5.4 & \text { Graduate/Alumni survey } \\
5.5 & \begin{array}{l}\text { Administration of new } \\
\text { intake student survey }\end{array} \\
5.6 & \begin{array}{l}\text { Focus groups of final } \\
\text { year students }\end{array}\end{array}$ & & & & & & \\
\hline $\begin{array}{l}\text { KC6 } \\
\text { Optimization of } \\
\text { resources and resource } \\
\text { issues for the program } \\
\text { and courses. }\end{array}$ & $\begin{array}{l}\text { Survey } \\
\text { tools }\end{array}$ & $\begin{array}{cl}6.1 & \begin{array}{l}\text { Review of Faculty } \\
\text { Qualifications }\end{array} \\
6.2 & \begin{array}{l}\text { Analysis of Faculty } \\
\text { attrition rates by Division }\end{array} \\
6.3 & \begin{array}{l}\text { Workload analysis of all } \\
\text { teaching loads }\end{array} \\
6.4 & \begin{array}{l}\text { Analysis of section size } \\
\text { and teaching ratios }\end{array} \\
6.5 & \begin{array}{l}\text { Physical teaching facilities } \\
\text { utilization analysis }\end{array} \\
6.6 & \begin{array}{l}\text { Alignment of PD activities } \\
\text { and allocation against } \\
\text { stated requirements }\end{array}\end{array}$ & $\begin{array}{l}\text { Employment } \\
\text { contracts. ACS- } \\
\text { faculty } \\
\text { allocation }\end{array}$ & & & & & \\
\hline
\end{tabular}




\begin{tabular}{|c|c|c|c|c|c|c|c|c|}
\hline & \multicolumn{3}{|c|}{ College responsibilities } & \multicolumn{5}{|c|}{ Program responsibilities } \\
\hline & Tools & Process & Data Gathering & Data Analysis & $\begin{array}{l}\text { Data } \\
\text { Interpretation }\end{array}$ & Action Planning & $\begin{array}{l}\text { Review of } \\
\text { Action Plan }\end{array}$ & Process/Review \\
\hline $\begin{array}{l}\text { Primary reporting group } \\
\text { (Link to) }\end{array}$ & $\begin{array}{l}\text { Does the } \\
\text { college } \\
\text { have } \\
\text { appropriate } \\
\text { tools to } \\
\text { collect data } \\
\text { against this } \\
\text { Key } \\
\text { Criterion? }\end{array}$ & $\begin{array}{l}\text { Does the college have a clear } \\
\text { administrative process } \\
\text { governing the use of these } \\
\text { tools? }\end{array}$ & $\begin{array}{l}\text { Has the college } \\
\text { followed its } \\
\text { admin } \\
\text { processes in the } \\
\text { collection of } \\
\text { data in this } \\
\text { current QA } \\
\text { cycle? }\end{array}$ & $\begin{array}{l}\text { Has the } \\
\text { collected data } \\
\text { been } \\
\text { analyzed by } \\
\text { program? }\end{array}$ & $\begin{array}{l}\text { Has the analysis } \\
\text { been interpreted } \\
\text { and conclusions } \\
\text { drawn resulting in } \\
\text { areas for action } \\
\text { being identified } \\
\text { and/or changes } \\
\text { made? }\end{array}$ & $\begin{array}{l}\text { For each identified } \\
\text { area for action, } \\
\text { has a plan been } \\
\text { developed to } \\
\text { address the } \\
\text { issues? }\end{array}$ & $\begin{array}{l}\text { Does a process } \\
\text { exist for the } \\
\text { review of } \\
\text { progress against } \\
\text { identified } \\
\text { actions in } \\
\text { previous plans? }\end{array}$ & $\begin{array}{l}\text { Has a review of the } \\
\text { effectiveness of the } \\
\text { process/tool used been } \\
\text { carried out? }\end{array}$ \\
\hline $\begin{array}{l}\text { KC7 } \\
\text { Course delivery and } \\
\text { teaching, learning and } \\
\text { assessment strategies: } \\
\text { Ensuring these are } \\
\text { aligned with KC3 }\end{array}$ & & $\begin{array}{cl}7.1 & \begin{array}{l}\text { Student Feedback on } \\
\text { teaching and learning }\end{array} \\
7.2 & \begin{array}{l}\text { Student feedback on } \\
\text { courses and materials }\end{array} \\
7.3 & \begin{array}{l}\text { Course team leader } \\
\text { reports }\end{array} \\
7.4 & \text { Course Assessment Files } \\
7.5 & \text { Teacher course feedback }\end{array}$ & & & & & & \\
\hline
\end{tabular}




\section{Appendix 5}

\section{College QA Data Responsibilities ‘05-’06 Chairs \& Program Staff}

\begin{tabular}{|c|c|c|c|c|c|c|c|}
\hline & & & & & Responsik & & \\
\hline & Tools & Process & Data (Evidence) & Data gathering & $\begin{array}{c}\text { Analysis/ } \\
\text { Interpretation }\end{array}$ & $\begin{array}{c}\text { Action } \\
\text { Plan/Report }\end{array}$ & $\begin{array}{l}\text { Data } \\
\text { Storage }\end{array}$ \\
\hline $\begin{array}{l}\text { KC1 } \\
\text { Stakeholder } \\
\text { satisfaction }\end{array}$ & $\begin{array}{l}\text { Survey tool } \\
\text { Work experience } \\
\text { assess form } \\
\text { Employer } \\
\text { feedback survey }\end{array}$ & $\begin{array}{ll}1.1 & \text { Survey of Employer/Industry } \\
\text { satisfaction with the program } \\
\text { 1.2 } & \text { Program Advisory Committees } \\
1.3 & \text { Employer Feedback on students } \\
1.4 & \text { College Forum }\end{array}$ & $\begin{array}{l}\text { Completed surveys } \\
\text { Database Raw \& collated data } \\
\text { PAC agendas/minutes } \\
\text { Completed surveys } \\
\text { Database Raw \& collated data } \\
\text { Agendas/minutes }\end{array}$ & & & & \\
\hline $\begin{array}{l}\mathrm{KC} 2 \\
\text { Professional and } \\
\text { external } \\
\text { benchmarking and } \\
\text { recognition (DAT) }\end{array}$ & & $\begin{array}{l}\text { 2.1 External Evaluation of Non-Accredited } \\
\text { Programs } \\
\text { 2.2 Review of Program Against Recognized } \\
\text { Standards }\end{array}$ & & & & & \\
\hline $\begin{array}{l}\text { KC3 } \\
\text { Alignment of } \\
\text { Academic Programs } \\
\text { and Support Service } \\
\text { Delivery with HCT } \\
\text { Graduate Outcomes } \\
\text { (DAT) }\end{array}$ & & $\begin{array}{l}\text { 3.1 Aligning Course Goals with Graduate } \\
\text { Outcomes } \\
\text { 3.2 Course Outline Review } \\
\text { 3.3 Course Audits } \\
\text { 3.4 Key Common Assessment Review } \\
\text { 3.5 Audit of Course Assessment Files }\end{array}$ & & & & & \\
\hline $\begin{array}{l}\text { KC4 } \\
\text { Student performance } \\
\text { in assessment and } \\
\text { progression through } \\
\text { the program. }\end{array}$ & $\begin{array}{l}\text { Grade sheets } \\
\text { Grade sheets }\end{array}$ & 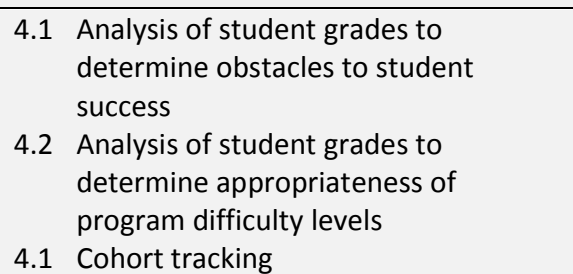 & $\begin{array}{l}\text { Raw data in Program grade } \\
\text { spreadsheets Banner system } \\
\text { Program analyses } \\
\text { Raw data in Program grade } \\
\text { spreadsheets Banner grade } \\
\text { system } \\
\text { Program analysis }\end{array}$ & & & & \\
\hline $\begin{array}{l}\text { KC5 } \\
\text { Student and graduate } \\
\text { satisfaction with }\end{array}$ & Survey tools & $\begin{array}{l}\text { 5.1 Student feedback on faculty teaching } \\
\text { and learning } \\
5.2 \text { Student Exit survey }\end{array}$ & $\begin{array}{l}\text { Completed survey docs } \\
\text { Completed survey docs }\end{array}$ & & & & \\
\hline
\end{tabular}




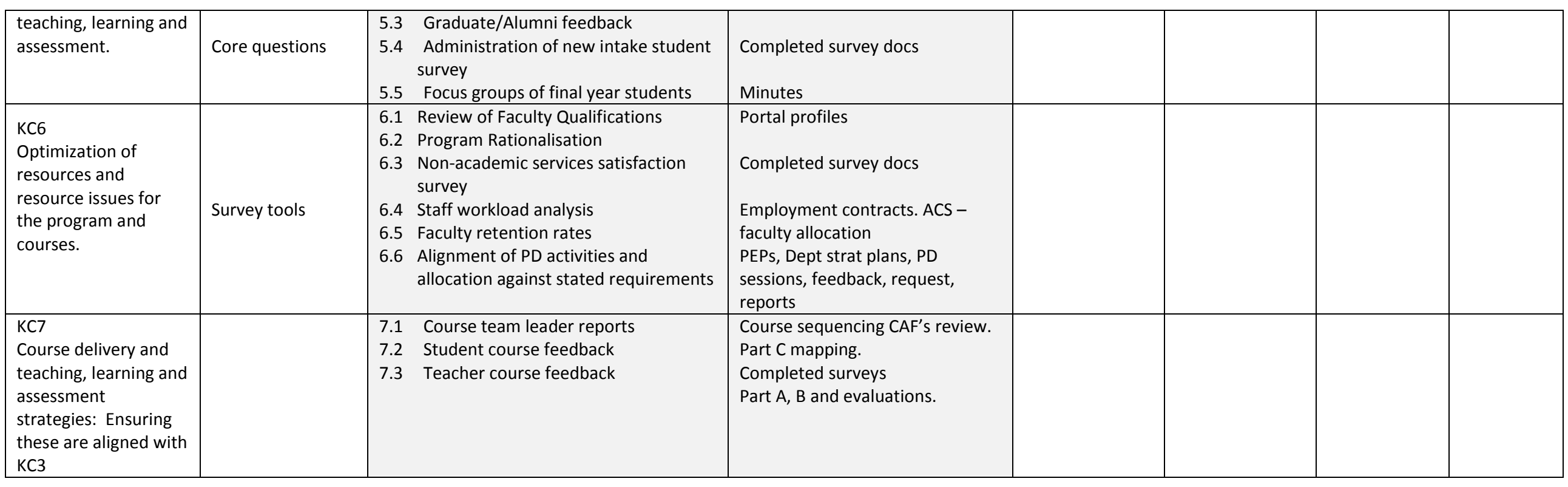




\section{Appendix 6}

\section{College Data Management Responsibilities 2005-2006}

\begin{tabular}{|c|c|c|c|c|c|c|c|}
\hline & Tools & Process & Data (Evidence) & Current Storage & Storage needs & $\begin{array}{c}\text { Current } \\
\text { Responsibility }\end{array}$ & $\begin{array}{c}\text { Planned } \\
\text { Responsibility }\end{array}$ \\
\hline $\begin{array}{l}\text { KC1 } \\
\text { Industry and } \\
\text { community } \\
\text { satisfaction with the } \\
\text { program }\end{array}$ & $\begin{array}{l}\text { Survey tool } \\
\text { Work } \\
\text { experience } \\
\text { assess form } \\
\text { Employer } \\
\text { feedback } \\
\text { survey }\end{array}$ & $\begin{array}{ll}1.1 & \text { Employer Survey } \\
1.2 & \text { Program Advisory } \\
\text { Committees (PACS) } \\
\text { 1.3 } & \begin{array}{l}\text { Employer Feedback on } \\
\text { students }\end{array} \\
1.4 & \begin{array}{l}\text { Employer feedback on } \\
\text { Graduates }\end{array} \\
1.5 & \text { College Forum }\end{array}$ & $\begin{array}{ll}\text { - } & \text { Completed } \\
& \text { surveys, raw \& } \\
& \text { collated data } \\
\text { - } & \text { PAC } \\
& \text { agendas/minutes } \\
\text { - } & \text { Completed surveys } \\
\text { - } & \text { Database raw \& } \\
& \text { collated data } \\
\text { - } & \text { Agendas/minutes }\end{array}$ & $\begin{array}{l}\text { H: Drive by Program } \\
\text { H:Drive Common }\end{array}$ & $\begin{array}{l}\text { Central College Data } \\
\text { repository }\end{array}$ & $\begin{array}{l}\text { Academic Chairs } \\
\text { CERC \& Academic } \\
\text { Chairs } \\
\text { Academic Dean }\end{array}$ & $\begin{array}{l}\text { QA Co-ord + } \\
\text { Program Admin } \\
\text { QA Co-ord + } \\
\text { CERC }\end{array}$ \\
\hline $\begin{array}{l}\text { KC4 } \\
\text { Student performance } \\
\text { in assessment and } \\
\text { progression through } \\
\text { the program. This } \\
\text { refer to processes } \\
\text { implemented at the } \\
\text { end of each semester. }\end{array}$ & Grade sheets & $\begin{array}{ll}\text { 4.1 An analysis of student } \\
\text { grades to determine } \\
\text { obstacles to student success } \\
\text { 4.2 Analysis of student grades to } \\
\text { determine the grades to } \\
\text { determine the } \\
\text { appropriateness of program } \\
\text { difficulty levels } \\
4.3 \text { Cohort attrition tracking } \\
4.4 \text { Monitoring ADW student } \\
\text { performance in relation to } \\
\text { other colleges (KCAs) } \\
\text { 4.5 Student performance in Gen } \\
\text { Ed by program }\end{array}$ & $\begin{array}{l}\text { - } \begin{array}{l}\text { Raw data in } \\
\text { Program grade } \\
\text { spreadsheets }\end{array} \\
\text { - } \quad \text { Banner grade } \\
\text { system }\end{array}$ & $\begin{array}{l}\text { H: Drive by Program } \\
\text { \& } \\
\text { Banner (central) } \\
\text { Ad hoc 'at risk' data? } \\
\text { location }\end{array}$ & $\begin{array}{l}\text { Central College } \\
\text { Application to access } \\
\text { relevant data from } \\
\text { Banner \& Central } \\
\text { College Data } \\
\text { repository }\end{array}$ & $\begin{array}{l}\text { Academic Chairs } \\
\text { Gen Ed Dean }\end{array}$ & $\begin{array}{l}\text { QA Co-ord, } \\
\text { College } \\
\text { Management \& } \\
\text { CS }\end{array}$ \\
\hline
\end{tabular}




\begin{tabular}{|c|c|c|c|c|c|c|c|}
\hline & Tools & Process & Data (Evidence) & Current Storage & Storage needs & $\begin{array}{c}\text { Current } \\
\text { Responsibility }\end{array}$ & $\begin{array}{c}\text { Planned } \\
\text { Responsibility }\end{array}$ \\
\hline $\begin{array}{l}\text { KC5 } \\
\text { Student and graduate } \\
\text { satisfaction with } \\
\text { teaching, learning and } \\
\text { assessment. }\end{array}$ & $\begin{array}{l}\text { Survey tools } \\
\text { Core questions }\end{array}$ & $\begin{array}{ll}5.1 & \text { Graduate survey } \\
5.2 & \text { Alumni survey } \\
5.3 & \text { Student survey } \\
5.4 & \text { Administration of new } \\
& \text { intake student survey } \\
5.5 \quad \text { Focus groups of final year } \\
\text { students }\end{array}$ & $\begin{array}{l}\text { Completed survey docs } \\
\text { Completed survey docs } \\
\text { Completed survey docs } \\
\text { Completed survey docs } \\
\text { Minutes }\end{array}$ & $\begin{array}{l}\text { H:Drive CERC } \\
\text { H: Drive by Program } \\
\text { Foundations only }\end{array}$ & $\begin{array}{l}\text { Central College Data } \\
\text { repository }\end{array}$ & $\begin{array}{l}\text { CERC } \\
\text { Academic Chairs } \\
\text { /Team Leaders }\end{array}$ & $\begin{array}{l}\text { QA Co-ord/ } \\
\text { CERC/ Program } \\
\text { Admin }\end{array}$ \\
\hline $\begin{array}{l}\text { KC6 } \\
\text { Optimization of } \\
\text { resources and } \\
\text { resource issues for } \\
\text { the program and } \\
\text { courses. }\end{array}$ & Survey tool & 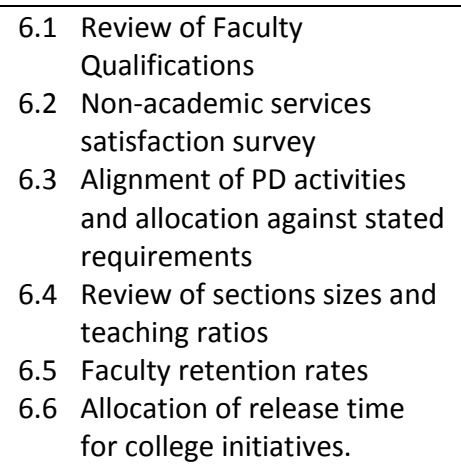 & $\begin{array}{l}\text { Portal profiles } \\
\text { Survey response data } \\
\text { PEPs, Dept strat plans, } \\
\text { PD sessions data, } \\
\text { feedback, requests, } \\
\text { reports }\end{array}$ & $\begin{array}{l}\text { Central ILE database } \\
\mathrm{H} \text { :Drive SS area } \\
\text { folders } \\
\text { College portal } \\
\text { H: Drive Ed Tech }\end{array}$ & $\begin{array}{l}\text { Nil } \\
\text { Central College Data } \\
\text { repository }\end{array}$ & $\begin{array}{l}\text { Academic Chairs } \\
\text { Not happening } \\
\text { Academic Chairs, Ed } \\
\text { Tech }\end{array}$ & $\begin{array}{l}\text { QA Co-ord/ } \\
\text { Program Admin } \\
\text { QA Co-ord } \\
\text { QA Co-ord \& } \\
\text { Academic Chairs }\end{array}$ \\
\hline $\begin{array}{l}\text { KC7 } \\
\text { Course delivery and } \\
\text { teaching learning and } \\
\text { assessment } \\
\text { strategies: Ensuring } \\
\text { these are aligned with } \\
\text { KC3 (Program and } \\
\text { course aims, design } \\
\text { and outcomes. }\end{array}$ & $\begin{array}{l}\text { Survey tool } \\
\text { Report format } \\
\text { Part A, B docs } \\
\text { CAFs } \\
\text { Course } \\
\text { feedback } \\
\text { survey }\end{array}$ & 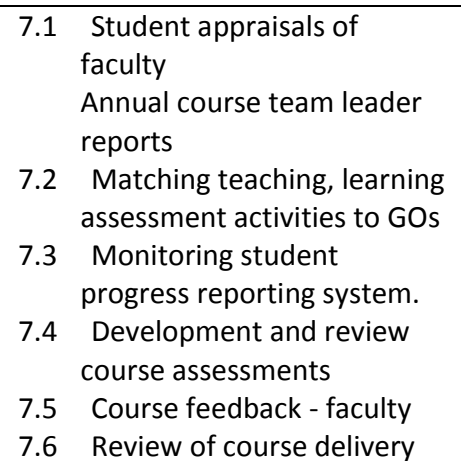 & $\begin{array}{l}\text { Raw \& collated survey } \\
\text { data } \\
\text { Teaching \& learning } \\
\text { docs, assess'ts, } \\
\text { resources, reports } \\
\text { Completed forms } \\
\text { Grade spread sheets } \\
\text { Banner grade system } \\
\text { Progress reports } \\
\text { Course Assess Files } \\
\text { Raw data }\end{array}$ & $\begin{array}{l}\text { H:drive CS } \\
\text { Hard copies teacher } \\
\text { file } \\
\text { New online tool } \\
\text { H:Drive by program } \\
\text { H:drive by program; } \\
\text { Banner (central); } \\
\text { H:Drive CS; College } \\
\text { portal }\end{array}$ & $\begin{array}{l}\text { Central College Data } \\
\text { repository } \\
\text { Central College Data } \\
\text { repository } \\
\text { Central College Data } \\
\text { repository }\end{array}$ & $\begin{array}{l}\text { CS } \\
\text { Team Leaders } \\
\text { Academic Chairs }\end{array}$ & $\begin{array}{l}\text { QA Co-ord \& } \\
\text { Prog Admin } \\
\text { QA Co-ord \& } \\
\text { team leaders } \\
\text { Academic Chairs } \\
\text { \& QA Co-ord }\end{array}$ \\
\hline
\end{tabular}




\section{Appendix 7}

\section{The problem situation themes}

\begin{tabular}{|c|c|}
\hline Themes & Comments/Data \\
\hline $\begin{array}{l}\text { Barriers/issues } \\
\text { constraining } \\
\text { effectiveness }\end{array}$ & 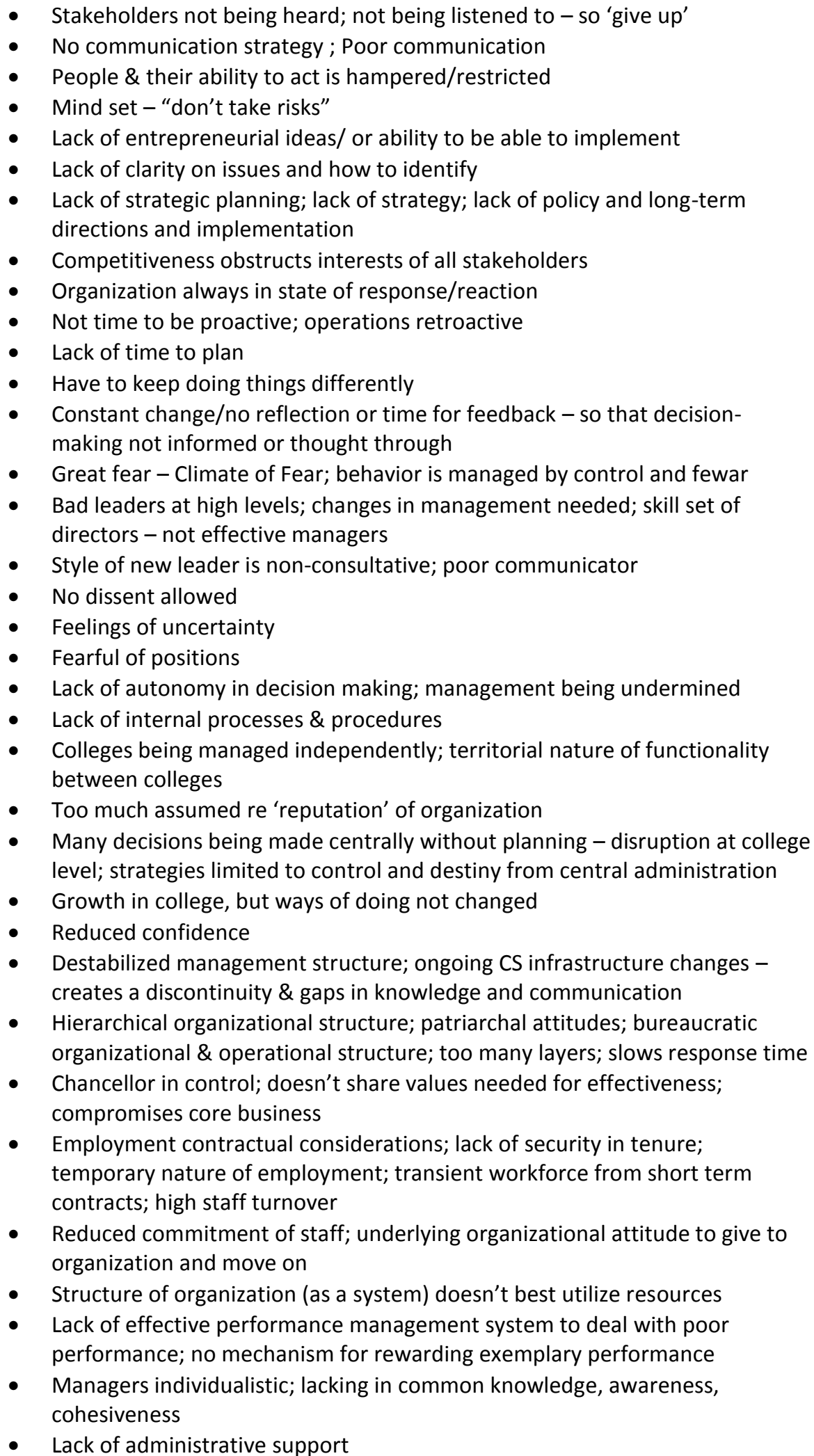 \\
\hline
\end{tabular}




\begin{tabular}{|c|c|}
\hline & $\begin{array}{l}\text { - } \quad \text { More management by 'walking' needed } \\
\text { - } \text { Maturity of organization reflects maturity level of country; rapidly developing } \\
\text { - } \quad \text { Rate of growth to change creates pressures on resources } \\
\text { - } \quad \text { Lack of time for consolidation or establishment of systems } \\
\text { - Need for review/audit } \\
\text { - } \quad \text { Leed for building new organization } \\
\text { - } \quad \text { Competing priorities } \\
\text { - } \quad \text { Lack of commitment } \\
\text { - Lack of clear vision that is articulated } \\
\text { - } \quad \text { Lack of KM systems; policies, procedures etc } \\
\text { - } \quad \text { Lack of transparency with management } \\
\text { - } \quad \text { Withlexibility in educational articulation } \\
\end{array}$ \\
\hline Policy/Strategy/KM & $\begin{array}{l}\text { - Retroactive operations } \\
\text { - } \text { Disconnections created when people move on } \\
\text { - } \quad \text { } \quad \text { KM - lack need to counteract knowledge loss } \\
\text { - Lack of policies, procedures, guidelines } \\
\text { - } \quad \text { Policies not well thought through - eg Emiritisation, concept OK, but no PD, } \\
\text { no training or mentor programs } \\
\text { - Lack of strategic plan } \\
\text { - } \quad \text { Lack of direction } \\
\text { - Tacit to explicit knowledge not being transferred } \\
\text { - Lack of clarity in processes \& policies }\end{array}$ \\
\hline Change & $\begin{array}{l}\text { - Individuals powerless to change } \\
\text { - } \quad \text { Need to make things happen rather than increasing control } \\
\text { - } \quad \text { Need to adapt to constant change } \\
\text { - } \quad \text { Bureaucratic change } \\
\text { - Cynicism to change } \\
\text { - Life changes faster here than anywhere else } \\
\text { - Student's culture is changing } \\
\text { - } \quad \text { Lack of PD in relation to QA and change } \\
\text { - } \quad \text { Ongoing, unplanned change, constant } \\
\text { - Impact of constancy in change causes 'let all wash over'; why bother?; } \\
\text { powerlessness, give up trying to be heard; lack of response to queries; } \\
\text { - } \quad \text { uncertainty; forced to work with change/ no choice } \\
\text { - Nothing allowed to run \& be analyzed } \\
\text { - Changing, changing, changing, so fast, no time for reflection, only quick } \\
\text { - Change needed to make things happen rather than increasing control } \\
\text { - Need for massive change in attitude of management } \\
\text { - Decision needed re whether change necessary or that it is OK to maintain } \\
\text { status quo }\end{array}$ \\
\hline
\end{tabular}




\begin{tabular}{|c|c|}
\hline Communication & $\begin{array}{l}\text { - } \quad \text { Poor communication, due to lack of appropriate leadership } \\
\text { - } \quad \text { Piecemeal communication; information not being passed on; working in the } \\
\text { - } \quad \text { Tark } \\
\text { - } \quad \text { Need for understanding of significance of communication } \\
\text { - } \quad \text { Need for strategy } \\
\text { - } \quad \text { Communication lacking, people unaware of what is going on; Lack of } \\
\text { - } \quad \text { Not on agenda as an issue; different priorities, communication low priority } \\
\text { - } \quad \text { Lack of follow-up } \\
\text { - } \quad \text { Traditional communication lines broken with staff/leadership changes } \\
\text { - } \quad \text { Communication systems remain static, no changes in ways of doing despite } \\
\text { - } \quad \text { changes in size of college } \\
\text { - } \quad \text { Fear-driven communication; fear of unknown } \\
\text { - } \quad \text { Leadership \& communication are keys to organizational effectiveness } \\
\text { - } \quad \text { Communication takes place through inappropriate channels } \\
\text { - } \quad \text { Lack of dialogue \& constructive conversation } \\
\text { - } \quad \text { Dood feedback systems \& mechanisms needed internally \& externally } \\
\text { - } \text { Multiple layers of organization create many opportunities to misinterpret }\end{array}$ \\
\hline Socio-cultural & 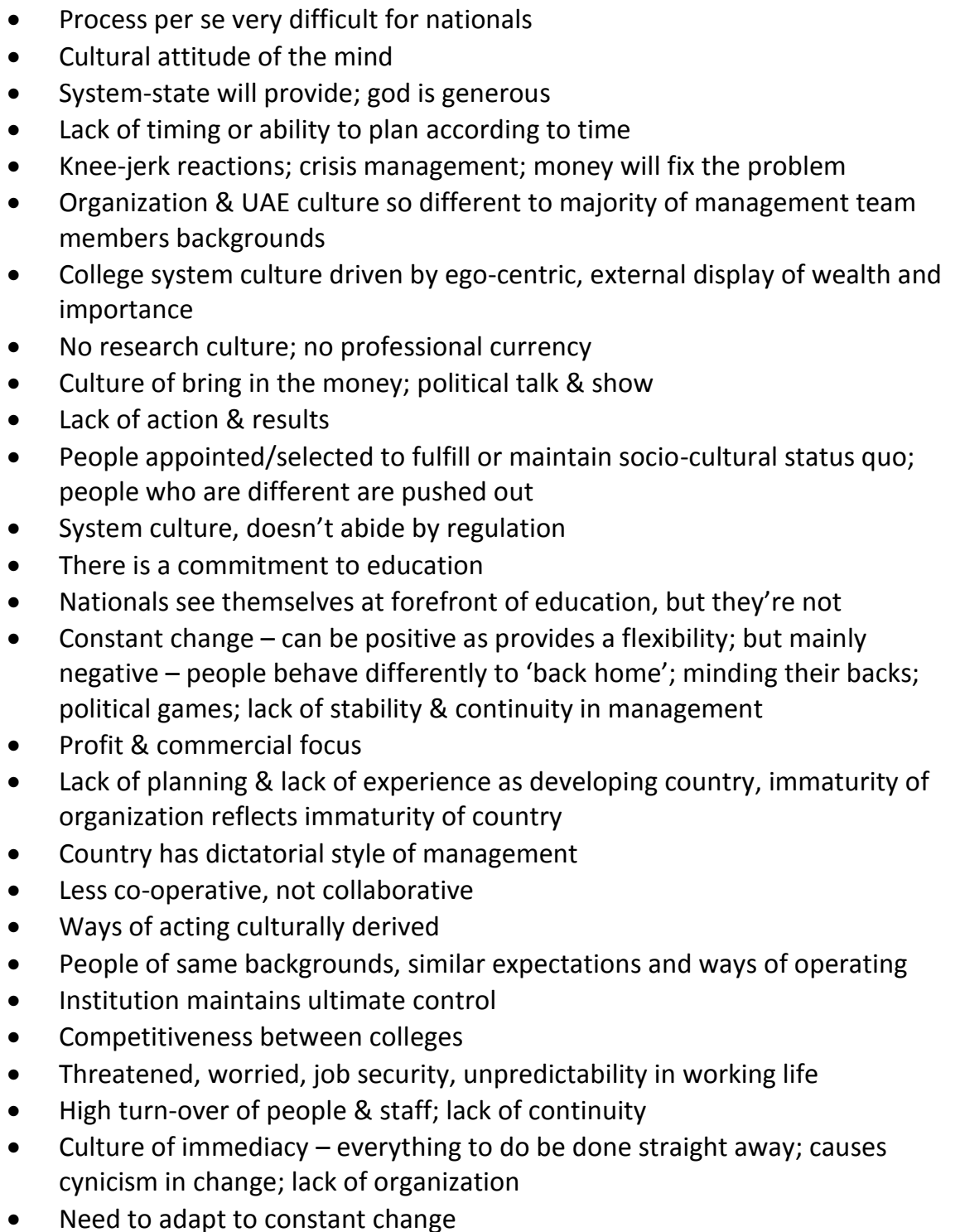 \\
\hline
\end{tabular}




\begin{tabular}{|c|c|}
\hline & 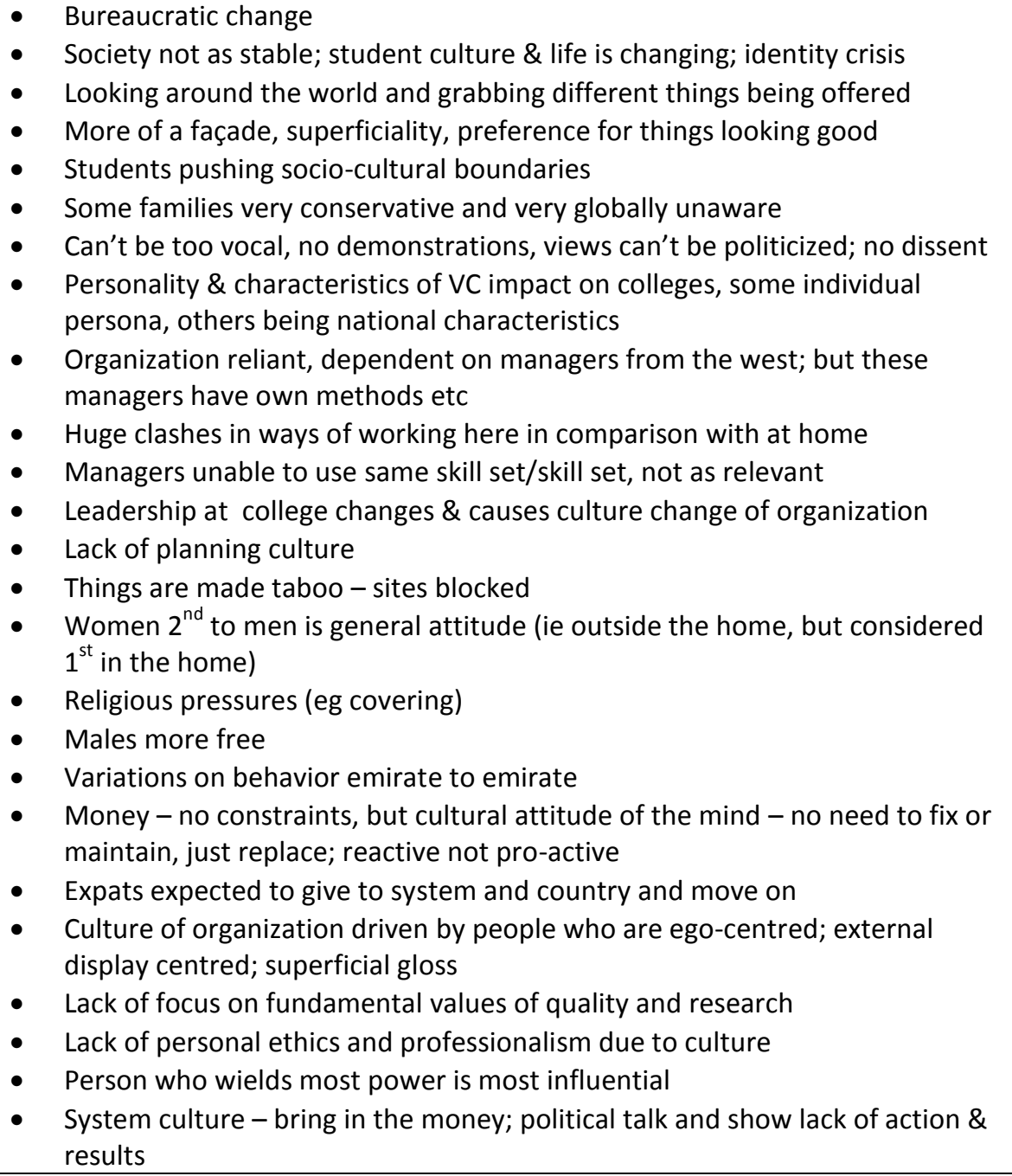 \\
\hline $\begin{array}{l}\text { Leadership \& } \\
\text { effectiveness }\end{array}$ & $\begin{array}{l}\text { - } \quad \text { The two are directly related } \\
\text { - } \quad \text { Effectiveness lacking due to leaders failing to set directions \& purposeful } \\
\text { - } \quad \text { Management culture, patriarchal, authoritarian, hierarchical, bureaucratic } \\
\text { - } \quad \text { Lack of appropriate leadership, causes poor communication } \\
\text { - } \quad \text { Attitude of management needs changing } \\
\text { - } \quad \text { Need for PD, management training, team building } \\
\text { - } \quad \text { Reluctance to assume leadership role } \\
\text { - } \quad \text { Authority not aligned with responsibility; } \\
\text { - } \quad \text { Leaders micromanage } \\
\text { - } \quad \text { Lack of clear direction; vague approach } \\
\text { - } \quad \text { Leader has different priorities } \\
\text { - } \quad \text { Need leader with vision, trustworthy, open-door, openness to change; } \\
\text { - } \quad \text { strong; treat others with respect; delegating appropriately } \\
\text { - } \quad \text { Leed leadership for effective team work; to achieve goals; to help individuals } \\
\text { - } \quad \text { Individuals powerless to change; limited empowerment, need more } \\
\text { - } \quad \text { Need leaders of communication } \\
\text { - } \quad \text { Change in leaders has broken processes of communication } \\
\text { - } \text { More collegiality needed; need to feel valued } \\
\text { - } \quad \text { Skill set for leadership can be learned } \\
\text { - } \quad \text { Deed for consultation } \\
\text { Leaders set agenda - style and nationality combined influence behavior; non- }\end{array}$ \\
\hline
\end{tabular}




\begin{tabular}{|c|c|}
\hline & 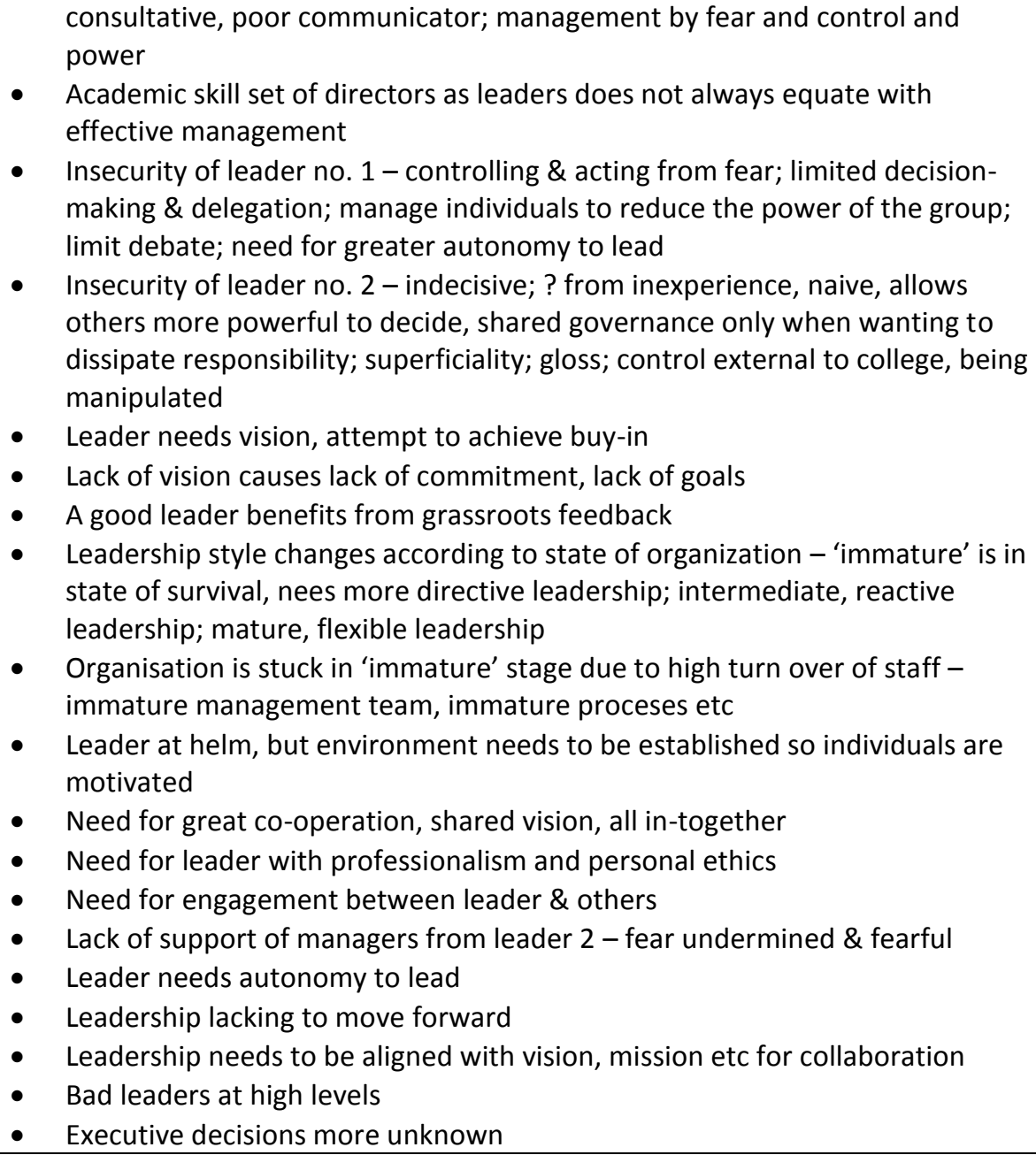 \\
\hline QA as project & 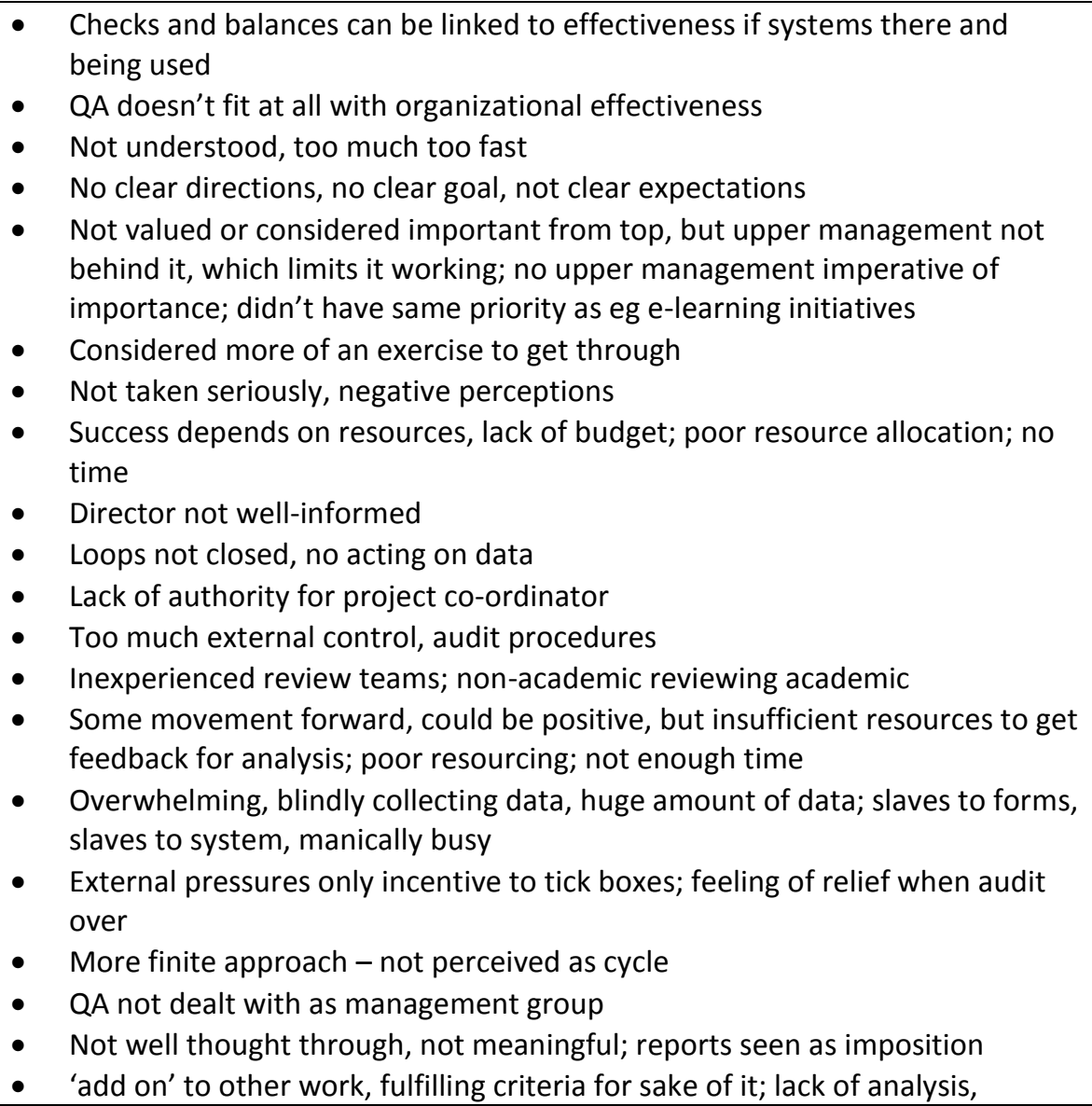 \\
\hline
\end{tabular}




\begin{tabular}{|c|c|}
\hline & 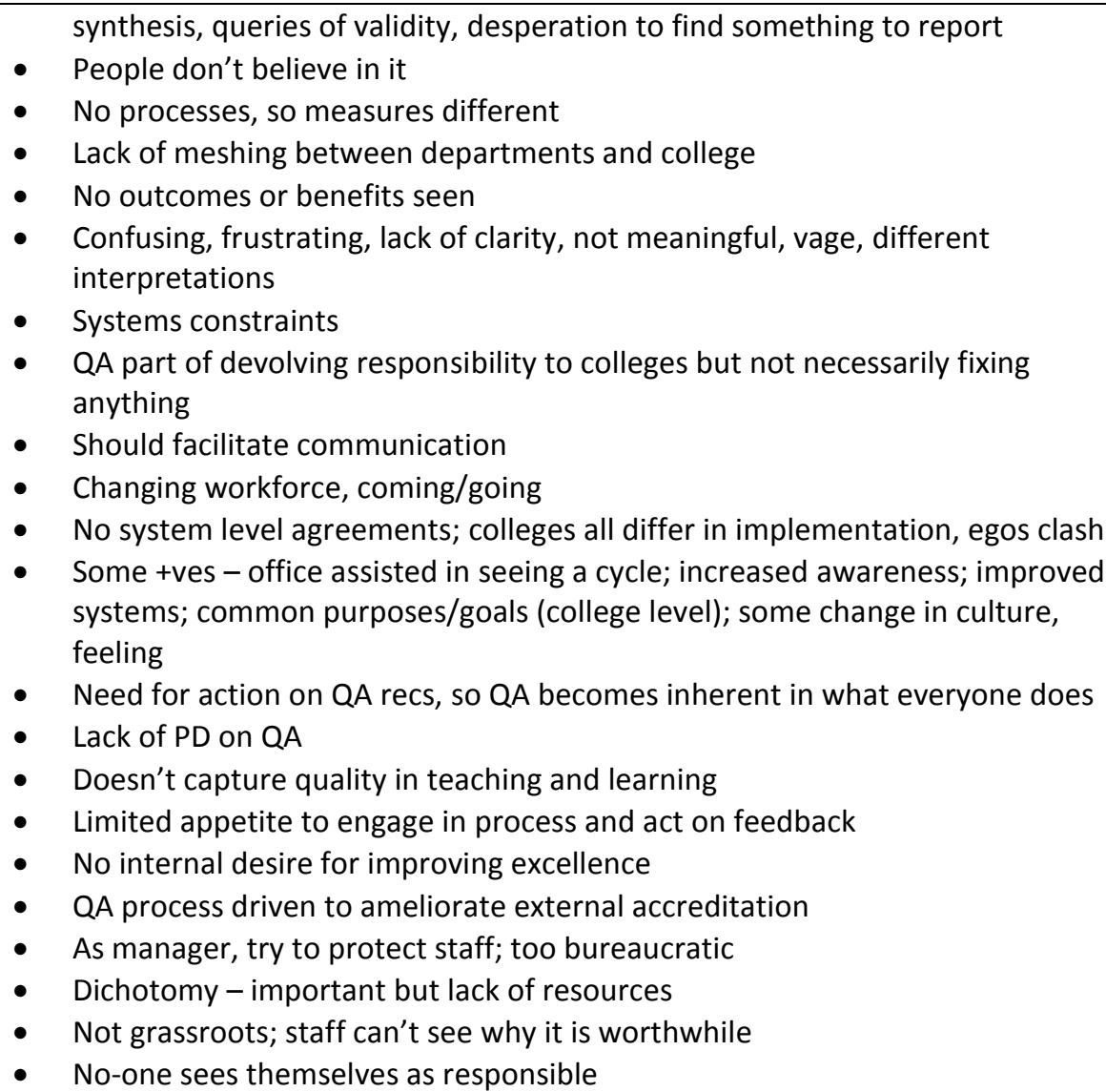 \\
\hline Stakeholders & $\begin{array}{ll}\text { - } & \text { Students } \\
\text { - } & \text { Families } \\
\text { - } & \text { Management team (mainly in relation to effectiveness) } \\
\text { - } & \text { Comployers, industry } \\
\text { - } & \text { Faculty }\end{array}$ \\
\hline
\end{tabular}




\begin{tabular}{|c|c|}
\hline \multicolumn{2}{|c|}{ Recommendations for increasing organizational effectiveness through change } \\
\hline Communication & $\begin{array}{l}\text { - Strategy needed to involve \& accommodate all stakeholders } \\
\text { - } \quad \text { Systems need to be non-static } \\
\text { - } \quad \text { More dialogue } \\
\text { - } \quad \text { Timstructive feedback reflection } \\
\text { - Strategy to accommodate different ways of communicating, } \\
\quad \text { different assumptions etc }\end{array}$ \\
\hline Knowledge management & $\begin{array}{l}\text { - Systems needed to capture knowledge and assist with knowledge } \\
\text { transfer } \\
\text { - Policy needed to counteract disconnections created when people } \\
\text { move on }\end{array}$ \\
\hline Leadership & $\begin{array}{l}\text { - } \quad \text { Visionary } \\
\text { - } \quad \text { Good communicator } \\
\text { - } \quad \text { Adle to articulate vision } \\
\text { - } \quad \text { Engaged with others } \\
\text { - Increased autonomy } \\
\text { - } \quad \text { Consult more with everyone for decision-making } \\
\text { - Transparency } \\
\text { - } \quad \text { Style of leadership needs to be changed }\end{array}$ \\
\hline Human element & $\begin{array}{l}\text { - Improve connect between manager and management group } \\
\text { - Staff need to be empowered } \\
\text { - Staff need to be consulted; more opportunity to contribute to } \\
\text { - } \quad \text { Secisions in relation to change } \\
\text { - Transience needs better management policies } \\
\text { - More PD, training and mentor programs }\end{array}$ \\
\hline Organisational strategy & $\begin{array}{l}\text { - } \quad \text { Planning - more long-term planning; more clarity } \\
\text { - Guidelines and specific directions } \\
\text { - Improve performance management systems } \\
\text { - } \quad \text { Articulated vision }\end{array}$ \\
\hline QA & $\begin{array}{l}\text { - } \quad \text { Values need to be incorporated into daily life } \\
\text { - } \quad \text { Should be linked with effectiveness } \\
\text { - } \quad \text { Meed clearer goals, directions, expectations, purpose } \\
\text { - } \quad \text { effect change, action needed on recommendations } \\
\text { - } \quad \text { Internal desire for improving excellence needed } \\
\text { - } \quad \text { More time to share experience to benefit } \\
\text { - Too many different \& competing priorities }\end{array}$ \\
\hline
\end{tabular}




\begin{tabular}{|l|l|}
\hline \multicolumn{2}{|l|}{ Identified factors difficult to manage } \\
\hline - Different perceptions of time \\
- Different styles of planning - predominance of short-term, crisis \\
- planning - ?how to keep on track; overall lack of planning \\
- Different ways of communicating \\
- Different assumptions \\
- Desire for system of governance that maintains control \\
- Constant change, much is unplanned, uncoordinated, not \\
- Strategic, discontinuity predominant \\
- Oichotomy - as most prefer a status quo - change burn out \\
- Reactive, not proactive \\
- Immaturity of organization and country \\
- Importance of external image, superficiality, having the 'best' of \\
- everything \\
- Dissent not permitted \\
- New generation is pushing socio-cultural boundaries \\
\hline
\end{tabular}




\section{Appendix 8}

\section{Cycle 4 data from informal meetings and email responses}

From the data, a number of main themes arose in relation to organisational effectiveness. These were: leadership, communication, learning for change, barriers to effectiveness and socio-cultural factors.

Three questions were raised via email and then further explored in informal meetings outside the workplace:

i) Do you think there is any particular style of leadership which is more effective and why?

- Current model is dictatorial - actions without explanation

- When dealing with Arabs - listening is dependent on how much do they actually respect you; they will listen more if you have a name with wasta, depends on who they are, perceptions of others.

- Focus more on big events - how visible is the event - how good does it make them look

- The more effective leaders are those who stand up for what they believe in; Previous college director did what he thought was right - that is a reflection of his background, experience, knowledge.

- New director - making wrong decisions, taking advice from those around him - this is seen as weakness. If his employment was terminated it would look bad for national VC because he appointed him and most changes to the college are the VCs decision.

- New director is letting people figure things out for yourself - this is seen as weakness;

- Leaders need to be seen to be decisive - if you admit you don't know, then it is seen as weak.

- Trying to be inclusive and empower people without making ultimate decision, is seen as weak.

- There is a need to be more dictatorial in the Arab world.

- Saving face underlies most communication.

- Leader is taking too many actions without finding out facts - knee-jerk action ??out of fear; Action based on false information

- Communication not honest; Keeping false front

- Leader (new director) asks for advice, but doesn't follow up. Makes people feel worthless, disempowered, not motivated 
- Old leader (director) - asks for advice, but made ultimate decision - gave feedback and reason if didn't follow it: resulted in more loyalty; politically smart

- Closed environment/lack of trust, comes from leader; not knowing why

- Nationals tend to respect their leaders if they get what they want; if they feel they have won

- Tribal ways of working - wasta / look to a paternalistic leader

ii) What strategies or approaches could facilitate improved communication when working with people from culturally diverse backgrounds?

- Old director asked opinion \& then followed up

- When nationals are in a minority or working alone with expats they tend to try and emulate expats

- Major problems with communication, not necessarily the nationalities. Different personalities are challenging and identifying differences is crucial. Main difference is the overlay of cultural differences. eg Indians - function very much on hierarchy; knowledge is power; don't give info away or you give power away; need to feel needed; but work very much on fear and blame. ie Power/Authority/Blame

- How to manage the above? Be conscious of differences; need to establish environment that promotes trust, need to make environment feel safer.

- Manager needs to be publicly clear, transparent, need to be more directive, need to give employees the OK/permission to make mistakes and act; employees need to feel important, valued

- Adding the different personalities to the different cultures $\rightarrow$ complexity

- If there is dysfunctionality in a group - combining different cultures and personalities adds layer of complexity. Having a team comprise different personalities is same as anywhere in the wolrd

- Leader needs to acknowledge upfront the differences

- Leader needs to create the environment

- Leader needs to pick the right blend of people 
iii) What strategies could be implemented to manage learning for organisational change given the socio-cultural environment?

- Negative outcome from superficial changes based on appearance

- Positive changes - people; environment; if people are able to do their best, the organisation will move forward

- Employees need to be and need to feel respected, well resourced - if not, won't put head up in case they 'get shot'; people won't take risks; become self-focussed; don't move forward

- Innovation and risk taking used to happen - people were listened to by managers

- Currently, no reason given for change; everything done at a whim - no strategic action - leads to uncertainty, feeling out of the loop, and can't be bothered - inertia

- Change is OK - but need to know reason; need to be communicated; need time to evaluate \& reflect

- Change is to reactionary, not proactive, therefore non-predictable

- Sometimes unpredictability can be challenging and rewarding, but not when it is constant and non-sensical; change for the sake of change doesn't work

- As manager - sometimes difficult to keep people motivated - sense of humor useful to stop cynicism \& burnout

- Managers create their own 'department culture' for survival - gives a sense of belonging

- The unpredictability, some things that would not be acceptable 'at home' are allowed 